Florida International University

FIU Digital Commons

$11-1-2019$

\title{
Corrosion Durability of a Nano-Particle Enriched Zinc-Rich Coating System For Highway Steel Bridges
}

\author{
Saiada Fuadi Fancy \\ Saiada Fuadi Fancy, sfanc002@fiu.edu
}

Follow this and additional works at: https://digitalcommons.fiu.edu/etd

Part of the Civil Engineering Commons, and the Structural Engineering Commons

\section{Recommended Citation}

Fancy, Saiada Fuadi, "Corrosion Durability of a Nano-Particle Enriched Zinc-Rich Coating System For Highway Steel Bridges" (2019). FIU Electronic Theses and Dissertations. 4344.

https://digitalcommons.fiu.edu/etd/4344

This work is brought to you for free and open access by the University Graduate School at FIU Digital Commons. It has been accepted for inclusion in FIU Electronic Theses and Dissertations by an authorized administrator of FIU Digital Commons. For more information, please contact dcc@fiu.edu. 


\section{FLORIDA INTERNATIONAL UNIVERSITY}

Miami, Florida

CORROSION DURABILITY OF A NANO-PARTICLE ENRICHED ZINC-RICH COATING SYSTEM FOR HIGHWAY STEEL BRIDGES

A dissertation submitted in partial fulfillment of

the requirements for the degree of

DOCTOR OF PHILOSOPHY

in

CIVIL ENGINEERING

by

Saiada Faudi Fancy 
To: Dean John L. Volakis

College of Engineering and Computing

This dissertation, written by Saiada Fuadi Fancy, and entitled Corrosion Durability of a Nano-Particle Enriched Zinc-Rich Coating System for Highway Steel Bridges, having been approved in respect to style and intellectual content, is referred to you for judgment.

We have read this dissertation and recommend that it be approved.

Norman D. H. Munroe

David Garber

Seung Jae Lee

Benjamin Boesl

Dale DeFord

Kingsley Lau, Major Professor

Date of Defense: November 1, 2019

The dissertation of Saiada Fuadi Fancy is approved.

Dean John L. Volakis College of Engineering and Computing

Andrés G. Gil

Vice President for Research and Economic Development and Dean of the University Graduate School

Florida International University, 2019 
(C) Copyright 2019 by Saiada Fuadi Fancy

All rights reserved. 


\section{DEDICATION}

I dedicate this dissertation to my beloved parents Md Abdus Salam and Mouloda

Begum. Their dream, inspiration, support, and most of all love encouraged me to complete this work. 


\section{ACKNOWLEDGMENTS}

Gratitude to Almighty Allah, the Most Merciful and the Gracious, for giving me the strength, knowledge, ability and opportunity to complete this dissertation.

First, I would like to express my heartiest gratitude to my major professor, Dr. Kingsley Lau, for his guidance, and support for this dissertation. He encouraged me to think independently, gave me room to work in my own way and try out new ideas. His approach and supervision encouraged me to do my best. His wide knowledge and logical way of thinking added invaluable input towards the completion of the dissertation.

I would like to express my deep and sincere gratitude to my committee members Dr. Norman D. H. Munroe, Dr. David Garber, Dr. Seung Jae Lee, Dr. Benjamin Boesl, and Dr. Dale DeFord for their motivation, guidance, and suggestion to improve my research work.

I would like to express my appreciation to my all lab mates of the Corrosion and Infrastructure Materials Durability Laboratory. Thanks to all caring friends for their friendship and memories. My acknowledgment also goes to all office staff of The Civil Engineering Department for their cooperation and help. I wish to extend my warmest thanks to all the colleagues, who have helped me with my work.

Last but not least, my deepest gratitude goes to my beloved husband and colleague, Md Ahsan Sabbir. Without his love, support, encouragement, and guidance it was not possible to finish this work. Not forgetting my lovely bothers, Tanvir Ahmed and Musabbir Ahmed for their love and care. Also, to those who indirectly contributed to this research, your kindness means a lot to me. Thank you very much. 


\title{
ABSTRACT OF THE DISSERTATION \\ CORROSION DURABILITY OF A NANO-PARTICLE ENRICHED ZINC-RICH \\ COATING SYSTEM FOR HIGHWAY STEEL BRIDGES
}

by

\author{
Saiada Fuadi Fancy \\ Florida International University, 2019 \\ Miami, Florida \\ Professor Kingsley Lau, Major Professor
}

Corrosion is a major concern for the long-term durability and structural integrity of steel components of highway bridges when unprotected. The application of protective coatings has been widely used for corrosion mitigation of atmospherically exposed structural steel. At present, the zinc-rich primer (ZRP) based three-coat system is widely used in the United States. The life of these coating systems is at best only half of the bridge design life. Furthermore, premature degradation may occur due to improper application. Different additives were considered to improve the performance of ZRP coating system and recently carbon nano-particles gathered attention due to their beneficial characteristics.

The protection mechanisms of zinc-rich coatings (sacrificial and barrier protection) have been well studied but the durability of zinc-rich coating containing carbon nano-particles has not been well elucidated for bridge application. In the work presented in this dissertation, a zinc-rich epoxy coating containing carbon nano-particles (NPE-ZRP) have been investigated for highway steel bridge application. Coating durability, robustness, and repair considerations in aggressive environments relevant to 
highway bridges were investigated. The research considered exposure to various environments to identify the degradation mechanism as well as the durability.

The NPE-ZRP coating initially provides barrier protection. The epoxy matrix allows electrolyte penetration from the exposure environment which facilitates the activation of the zinc pigments (cathodic protection) and the associated formation of zinc oxide further enhanced the barrier protection. Comparatively, improved barrier performance was observed for the NPE-ZRP coating system even with fewer coating layers. Similar galvanic protection as conventional ZRP was observed. Comparatively faster corrosion rates of NPE-ZRP also portray enhanced continuity through carbon the nano-particles. Higher pull-off strength was observed for NPE-ZRP coating apparently due to carbon nano-particles which enhanced the cohesive bond and the adhesive strength. Pre-exposure to high humidity didn't affect the coating durability but salt contamination and remnant coating layer can hinder the bond of the NPE-ZRP primer with the steel substrate. Most importantly, NPE-ZRP coating always showed zinc consumption from the bulk primer layer whereas ZRP showed along with the steel/primer interface. Eventually, NPE-ZRP maintained good bond strength whereas ZRP loses bond strength at the steel/primer interface. 


\section{TABLE OF CONTENTS}

CHAPTER

PAGE

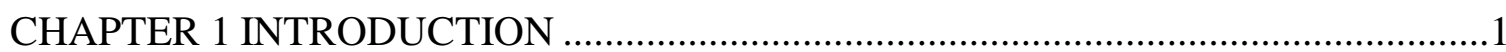

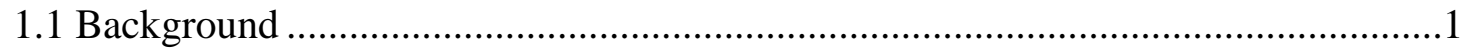

1.2 Problem Statement and Research Objective ...........................................................

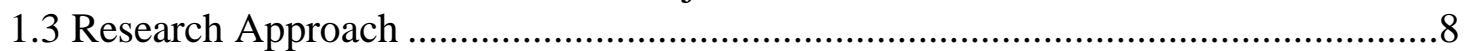

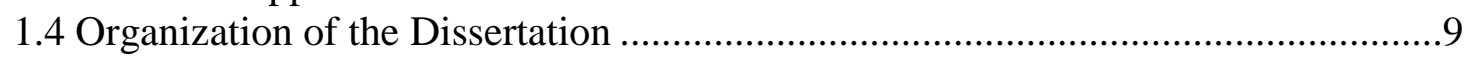

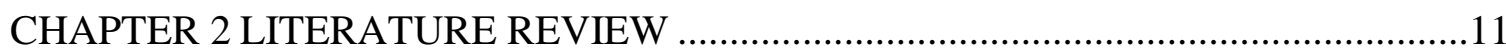

2.1 Steel Bridges and Service Environment.............................................................11

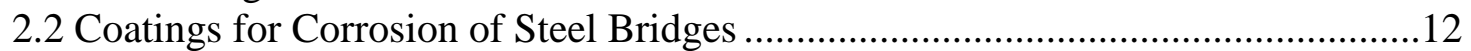

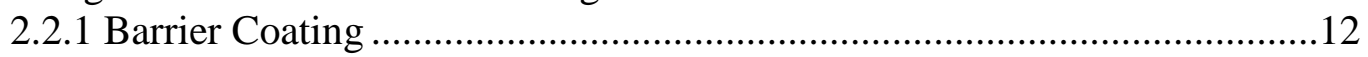

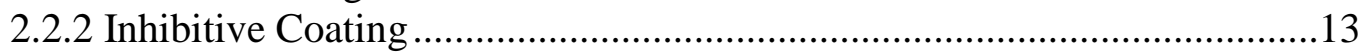

2.2.3 Sacrificial Coating ..................................................................................13

2.3 Present Practice of Bridge Coating System .........................................................14

2.3.1 Paint Coating System (Zinc-rich Three-coat system) .................................14

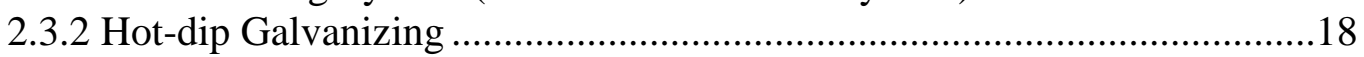

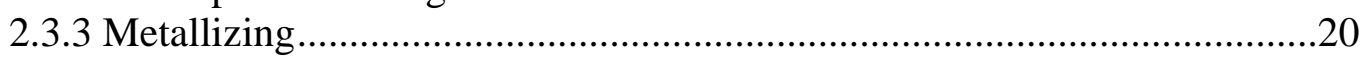

2.4 FDOT Steel Bridge Performance ……………………...................................21

2.4.1 Steel Bridges of Florida ..........................................................................21

2.4.2 FDOT Bridge Coating Performance ..........................................................22

2.4.3 FDOT Bridge Coating Maintenance Cost ...............................................26

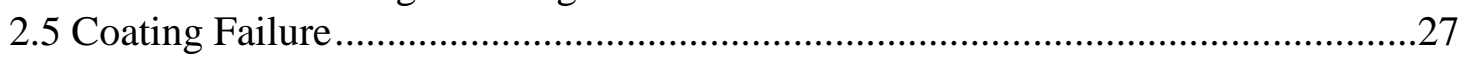

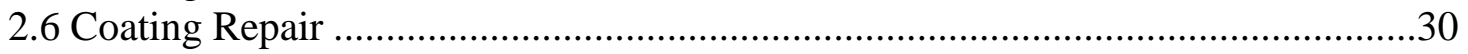

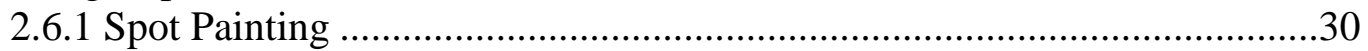

2.6.2 Overcoating ..........................................................................................

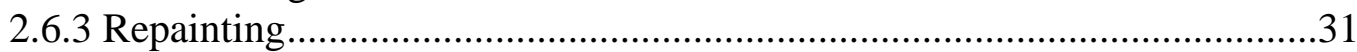

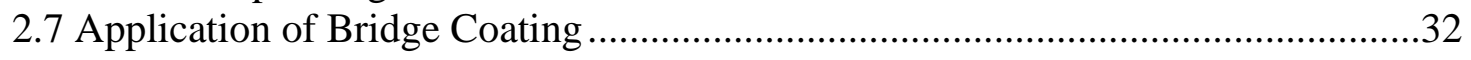

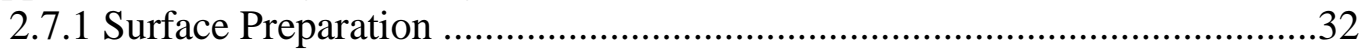

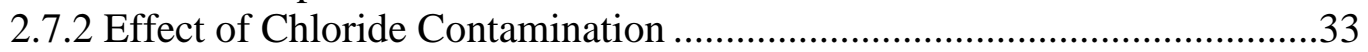

2.7.3 Effect of Relative Humidity and Moisture ..................................................35

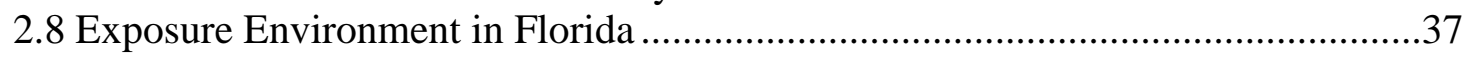

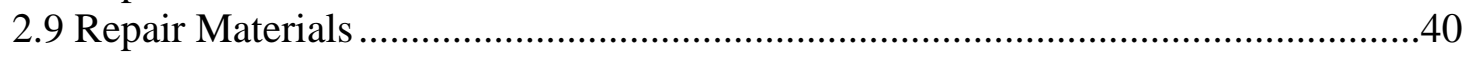

2.10 Improvement of ZRP Coating System ……………........................................4

2.10.1 Nano-particle Enriched ZRP (NPE-ZRP) Coating .................................42

2.10.2 Performance of NPE-ZRP Coating ..........................................................43

2.11 Electro Chemical Basic Principles .....................................................................4

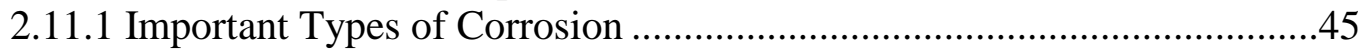

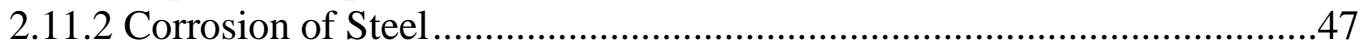

2.11.3 Corrosion of Zinc ………………..........................................................

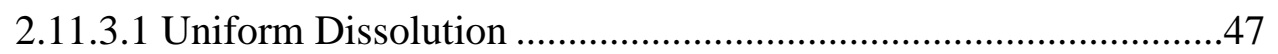

2.11.3.2 Wet Storage Stain ("White Rust") .............................................48

2.11.3.3 Galvanic Corrosion ..................................................................48 


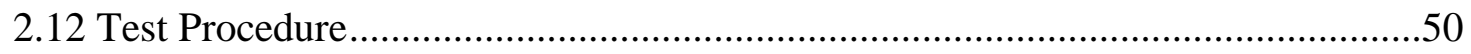

2.12.1 Three-electrode System ...................................................................50

2.12.2 Open Circuit Potential (OCP) ..............................................................50

2.12.3 Linear Polarization Resistance (LPR) ...................................................51

2.12.4 Electrochemical Impedance Spectroscopy ……………..........................51

2.12.5 Surface Wetting Property …………………………………………….....56

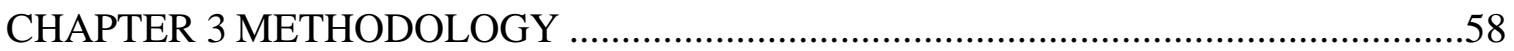

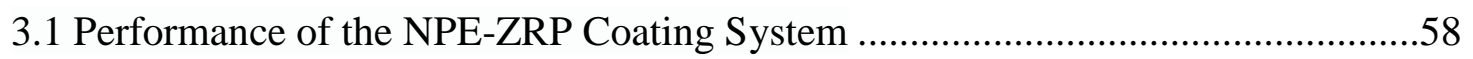

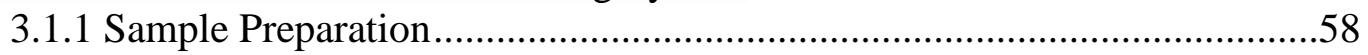

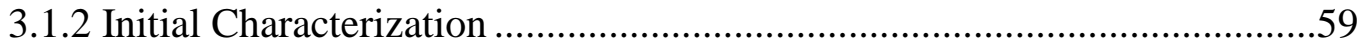

3.1.3 Long-term Exposure ...............................................................................60

3.1.3.1 Atmospheric Outdoor Exposure …………………….................60

3.1.3.2 Aggressive Salt-Fog Exposure.....................................................62

3.1.4 Laboratory Electrochemical Testing …………………………………....63

3.1.4.1 Water Immersion ....................................................................63

3.1.4.2 Electrochemical Polarization .........................................................64

3.2 NPE-ZRP for Application of Repair Coating ……...........................................65

3.2.1 Sample Preparation..............................................................................66

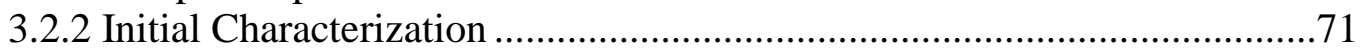

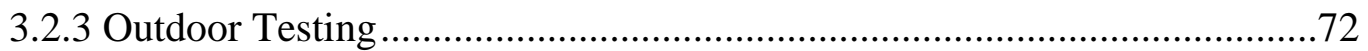

3.2.4 Cyclic Wet/Dry Exposure........................................................................73

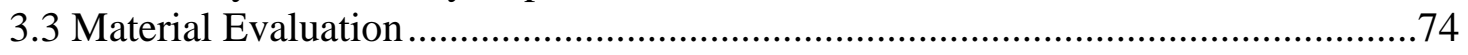

CHAPTER 4 PERFORMANCE OF THE NPE-ZRP COATING SYSTEM …….............78

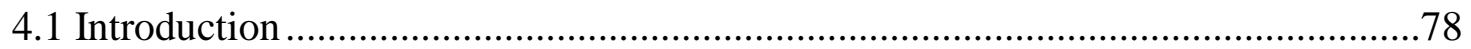

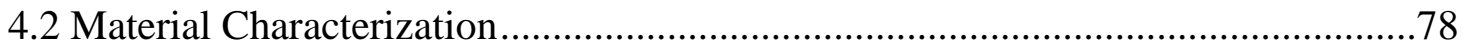

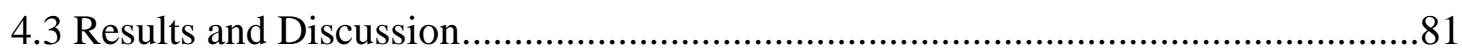

4.3.1 Atmospheric Outdoor and Salt-Fog Exposure ……….............................

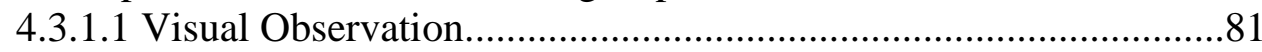

4.3.1.2 Coating Thickness.....................................................................83

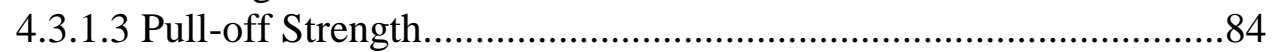

4.3.1.4 Characterization of Zinc Consumption .........................................85

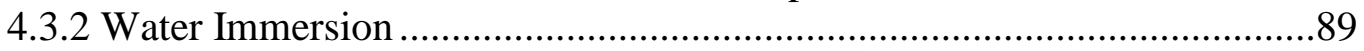

4.3.2.1 Electrochemical Analysis (OCP-LPR) ………………………....90

4.3.2.2 Electrochemical Impedance Spectroscopy (EIS) ...........................92

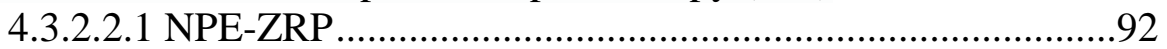

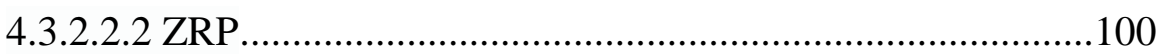

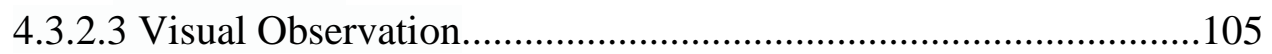

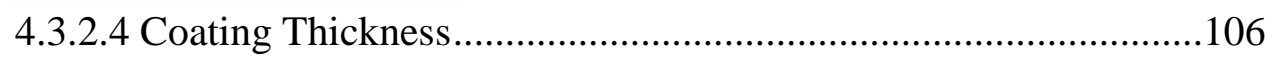

4.3.2.5 Pull-off Strength Test.................................................................107

4.3.2.6 Characterization of Zinc Consumption .......................................108

4.3.3 Electrochemical Polarization...............................................................109

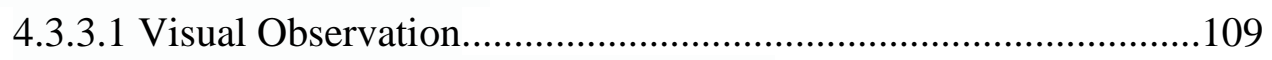

4.3.3.2 Electrochemical Analysis (OCP-LPR) ……………………........110 
4.3.3.3 Polarization Behavior...............................................................113

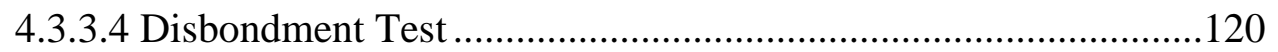

4.3.3.5 Pull-off Strength Test.................................................................123

4.3.3.6 Characterization of Zinc Consumption ........................................124

\section{CHAPTER 5 ROBUSTNESS OF NPE-ZRP COATING FOR REPAIR}

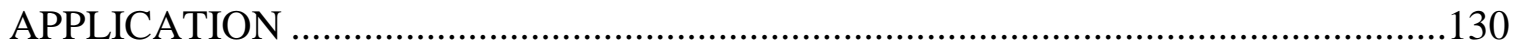

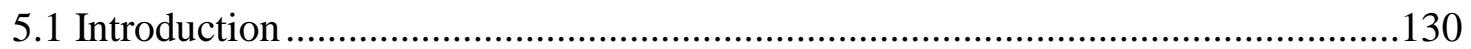

5.2 Steel Substrate Characterization before Coating Application................................131

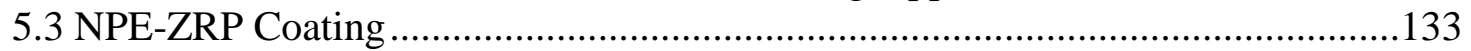

5.3.1 Material Characterization …………………………………………....133

5.3.2 Results and Discussion ......................................................................138

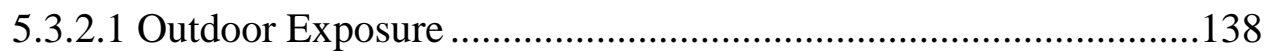

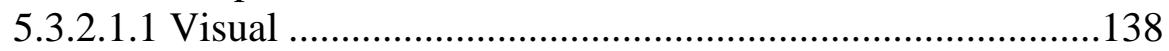

5.3.2.1.2 Coating Thickness .........................................................140

5.3.2.1.3 Pull-off Strength Test ......................................................141

5.3.2.2 Cyclic Exposure ………………………………………….......142

5.3.2.2.1 Visual Observation ......................................................142

5.3.2.2.2 Coating Thickness ...........................................................144

5.3.2.2.3 Pull-off Strength Test ......................................................147

5.3.2.4 Electrochemical Analysis (OCP-LPR) …………......................147

5.3.2.5 Characterization of Zinc Consumption .........................................152

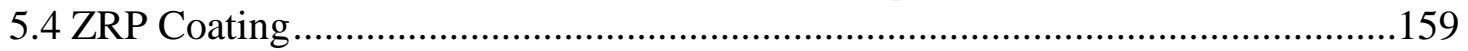

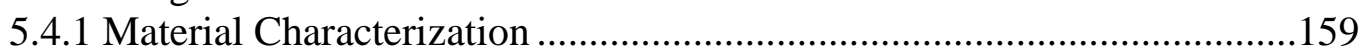

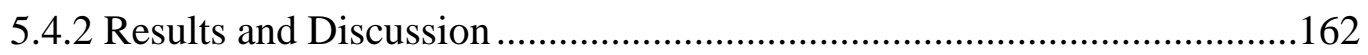

5.4.2.1 Outdoor Exposure ………………………………..................162

5.4.2.1.1 Visual Observation .......................................................162

5.4.2.1.2 Coating Thickness ............................................................163

5.4.2.1.3 Pull-off Strength Test ....................................................164

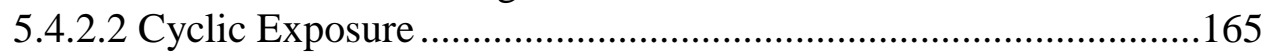

5.4.2.2.1 Visual Observation .......................................................165

5.4.2.2.2 Coating Thickness ..........................................................166

5.4.2.2.3 Pull-off Strength Test .......................................................168

5.4.2.4 Electrochemical Analysis (OCP-LPR) ........................................169

5.4.2.5 Characterization of Zinc Consumption .......................................172

CHAPTER 6 IMPROVEMENTS IN COATING DURABILITY BY

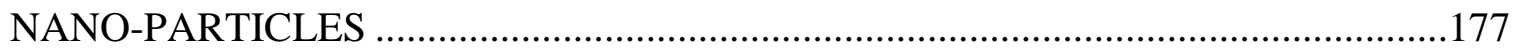

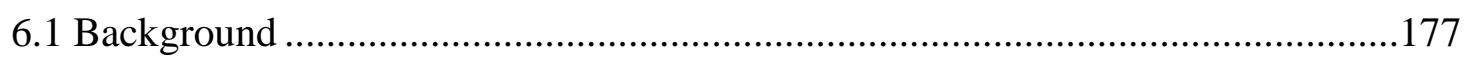

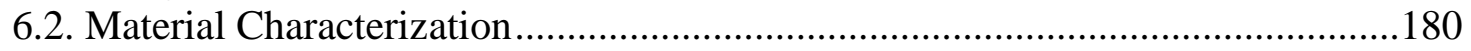

6.3 Coating Durability by Surface Measurements ……...........................................183

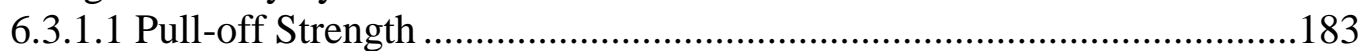

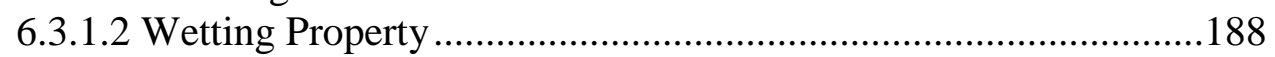

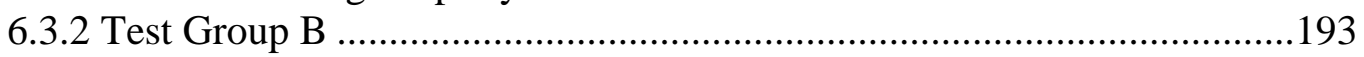

6.3.2.1 Pull-off Strength.........................................................................193 
6.3.2.2 Wetting Property .194

6.4 Statistical Approach to Find Coating Durability................................................196

CHAPTER 7 ZINC CONSUMPTION MECHANISM ............................................203

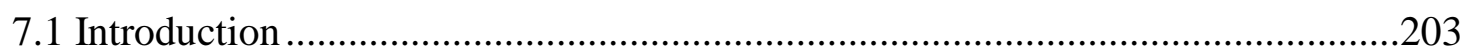

7.2 Role of Nano-Particles on Barrier and Cathodic Protection ..............................203

7.2 Role of Nano-Particles on Coating Bond Strength ...........................................207

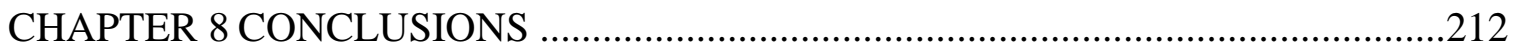

RECOMMENDATIONS FOR FUTURE RESEARCH............................................214

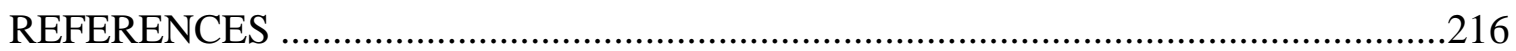

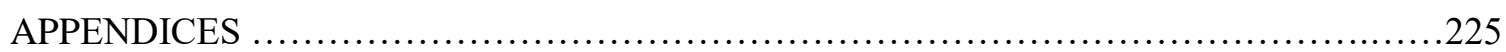

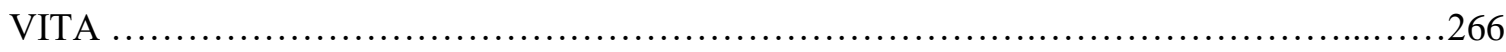




\section{LIST OF TABLES}

TABLE

PAGE

Table 2.1. Corrosion Rates of Carbon Steel in Different Environmental Exposure. .12

Table 2.2. Formulation Related Failures (Bayer \& Zamanzadeh, 2004)..........................28

Table 2.3. Adhesion Related Failures (Bayer \& Zamanzadeh, 2004) .............................29

Table 2.4. Substrate Related Failures (Bayer \& Zamanzadeh, 2004)..............................29

Table 2.5. List of SSPC and NACE Standards Specifications for Surface Finish. ...........33

Table 2.6. Electrode Potential of Metals in Seawater (Jones, 1996). .............................49

Table 3.1. Chemical Composition of the Low Carbon Steel Panels..................................59

Table 3.2. Pre-coating Surface Preparation. ................................................................. 70

Table 3.3. Alternate Wet/Dry Exposure Sequence (20 Cycles)....................................73

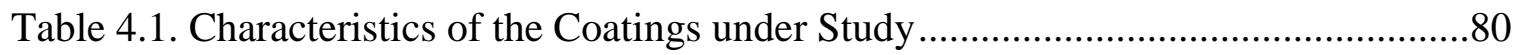

Table 4.2. Comparative Mass Loss After Exposure ......................................................119

Table 4.3. Zinc Consumption Characterization of Both Coatings................................128

Table 5.1. Zinc Consumption Characterization of NPE-ZRP Scribed Samples..............156

Table 5.2. Zinc Consumption Characterization of ZRP Scribed Samples......................175

Table 6.1. Characteristics of the Coatings under Study...........................................182

Table 6.2. Contact Angle of NPE-ZRP Sample without Topcoat. .................................194 


\section{LIST OF FIGURES}

FIGURE

PAGE

Figure 2.1. Metallographic Cross-section of Three-coat System. ..................................16

Figure 2.2. Metallographic Cross-section of Hot-dip Galvanizing (Sabbir, 2017). ..........19

Figure 2.3. Thermal Spray Coating, Zinc on Steel (by Lau, K. \& courtesy of FDOT).....21

Figure 2.4. Location of Florida Steel Bridges........................................................22

Figure 2.5. Age Distribution of Florida Steel Bridges................................................22

Figure 2.6. Age for Bridge Paint Repair (FDOT Bridge Inspection Report). ..................23

Figure 2.7. Age Distribution of Repainted Bridges (FDOT Bridge Work Plan 08-18).....23

Figure 2.8. Bridge Condition State over Age (FDOT Bridge Inspection Report)............24

Figure 2.9. Condition State over the Service Life (FDOT Bridge Inspection Report).....25

Figure 2.10. Number of Repainted Bridges (FDOT Bridge Work Plan 08-18). ..............26

Figure 2.11. Listed Repair Program for Year 2018 Bridge Work Plan..........................27

Figure 2.12. FDOT Cost Spent for Steel Bridge Repainting (Work Plan 08-18)............27

Figure 2.13. Experimental Threshold Chloride Level Data (Fuente et. al., 2006)............35

Figure 2.14. Environmental Classification of Structures (FDOT, 2017).........................38

Figure 2.15. Chloride Ion Deposition and Concentration, (NADP, 2015).......................39

Figure 2.16. Exposure Environment based on Chloride Exposure.................................39

Figure 2.17. Corrosion Rate with Distance from the Ocean (Morrison, 1980). ...............40

Figure 2.18. Typical Painting Systems Used for Maintenance (TRS, 2014)..................41

Figure 2.19. Structure of Carbon Nano-particles (Zhang, el. Al., 2010)........................42

Figure 2.20. Nano-Particle Enriched Zinc-Rich Primer (NPE-ZRP) Coating..................42

Figure 2.21. Equivalent Circuit of an Intact Coating in Contact with Electrolyte.............52 
Figure 2.22. Equivalent Circuit of Coating in Contact with Electrolyte by Considering the Base Property of the Steel.

Figure 2.23. Idealized Impedance Diagram and Equivalent Circuit of Coated Metal

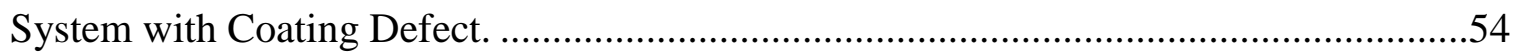

Figure 2.24. Energy Balance of a Liquid Drop Resting on a Solid Surface. ....................56

Figure 3.1. Location of Outdoor Exposure Sites. ....................................................61

Figure 3.2. Weather Data for Outdoor Test Sites. .................................................61

Figure 3.3. Test setup for Salt-fog Exposure. ........................................................62

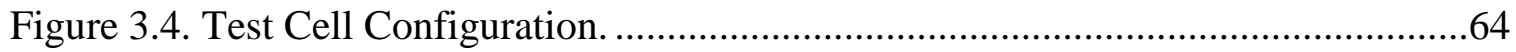

Figure 3.5. Laboratory Test Set-up for Potentiostatic Polarization. ...............................65

Figure 3.6. Sandblasting of the Coupons (Plain Steel Coupon). ..................................67

Figure 3.7. Surface Preparation by Grinding of Three-coat System..............................67

Figure 3.8. Hand Cleaned Steel Panels (Three-coat System). .......................................68

Figure 3.9. Measurement of the Chloride Contamination Level. ..................................69

Figure 3.10. Humidity Chamber for Test Panel Exposure...........................................69

Figure 3.11. Sample Exposure after Repair......................................................... 71

Figure 3.12. Outdoor Sample Exposure at FIU. .................................................. 72

Figure 3.13. Weather Data for FIU Test Site....................................................... 72

Figure 3.14. Cyclic Wet/Dry Exposure in Three Different Condition (20 Cycles)...........73

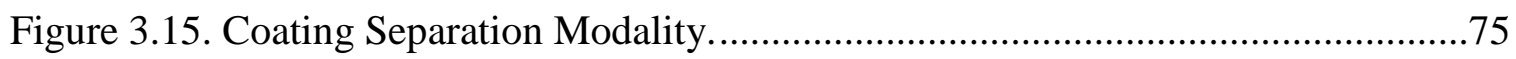

Figure 4.1. Metallographic Cross-sections of Test Coatings.......................................78

Figure 4.2. Zinc Particle Size Distribution in the Polymer Matrix.................................79

Figure 4.3. Different Coating Failure Modality...........................................................81 
Figure 4.4. Visual Appearance of the Outdoors and Salt-fog Exposed Samples. .82

Figure 4.5. Coating Thickness of Outdoor and Salt-fog Exposed Samples over Time.....84

Figure 4.6. Pull-Off Strength of Outdoor \& Salt-fog Exposed Samples..........................85

Figure 4.7. Degradation of the Outdoor and Salt-fog Exposed Unscribed Samples. ........86

Figure 4.8. Zinc Consumption of Outdoor \& Salt-fog Exposed Unscribed Samples........87

Figure 4.9. Degradation of the Exposed Scribed Samples (24 months Exposure)...........88

Figure 4.10. Open Circuit Potential during the Exposure............................................91

Figure 4.11. Corrosion Current over Exposure Duration. ...........................................91

Figure 4.12. EIS response of NPE-ZRP Unscribed sample (1MHz to $1 \mathrm{~Hz})$...................93

Figure 4.13. EIS response of NPE-ZRP Unscribed sample (100 kHz to $1 \mathrm{mHz}) \ldots \ldots \ldots \ldots . . .94$

Figure 4.14. Equivalent Circuit for EIS Analysis of NPE-ZRP Unscribed Sample.........95

Figure 4.15. Equivalent Circuit Elements for NPE-ZRP Unscribed Samples.................98

Figure 4.16. EIS response of NPE-ZRP Scribed sample (100 kHz to $1 \mathrm{mHz}) \ldots \ldots \ldots \ldots \ldots . . . .99$

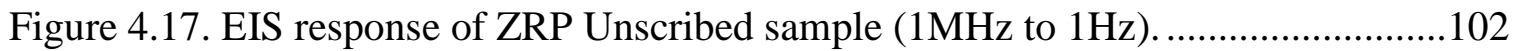

Figure 4.18. EIS response of ZRP Unscribed sample (100 kHz to $1 \mathrm{mHz})$..................103

Figure 4.19. EIS response of ZRP Scribed sample (100 kHz to $1 \mathrm{mHz}) \ldots \ldots \ldots \ldots \ldots \ldots \ldots . . . . . . . . . .104$

Figure 4.20. Condition of the Water Immersed Samples...........................................106

Figure 4.21. Change in Coating Thickness after Water Immersion. ............................107

Figure 4.22. Pull-off Strength and Failure Modality of Samples after Immersion.........108

Figure 4.23. Degradation of the Water Immersed Samples......................................108

Figure 4.24. Condition of the Representative Samples before and after Exposure. ........110

Figure 4.25. OCP\& LPR evolution of NPE-ZRP and ZRP with Time.........................112

Figure 4.26. OCP\& LPR evolution of NPE-ZRP and ZRP with Time..........................112 
Figure 4.27. Cathodic Current over Time for NPE-ZRP and ZRP Coating. .114

Figure 4.28. Anodic Current over Time for NPR-ZRP and ZRP Coating......................114

Figure 4.29. Cumulative Cathodic Charge for NPE-ZRP and ZRP Coating.................116

Figure 4.30. Cumulative Anodic Charge for NPE-ZRP and ZRP Coating. ...................117

Figure 4.31. Steel Corrosion after Anodic Polarization $\left(-300 \mathrm{mV}_{\mathrm{SCE}}\right) \ldots \ldots \ldots \ldots \ldots \ldots \ldots \ldots . . . . . . . . . . .118$

Figure 4.32. Disbondment of Samples after Exposure ...........................................121

Figure 4.33. Metallographic Cross-section of Exposed Samples to - $1500 \mathrm{mV}$................121

Figure 4.34. Metallographic Cross-section of Exposed Samples to $-600 \mathrm{mV}$..................123

Figure 4.35. Pull-off Strength after Exposure to Various Polarization Regimes.............124

Figure 4.36. X-Ray Diffractogram for the sample after exposure ..............................125

Figure 4.37. Zinc Consumption along the $300 \mu \mathrm{m}$ from Defect Site. ............................126

Figure 4.38. Effective Zinc Consumption per Unit Thickness along the 5mm Length...126

Figure 4.39. Zinc Consumption Modality after Exposure.........................................127

Figure 5.1. Condition of Uncoated Steel Test Panels after Exposure............................131

Figure 5.2. X-Ray Diffractogram for the Steel Plate after exposure. ............................133

Figure 5.3. Representative Surface Condition of Repaired Test Coupons. .....................133

Figure 5.4. Micrographs of Repaired NPE-ZRP Samples with Sandblasted Steel..........135

Figure 5.5. Micrographs of Repaired NPE-ZRP Samples with Handcleaned Steel........135

Figure 5.6. Coating Thickness of NPE-ZRP Coupons after Repair. .............................136

Figure 5.7. Coating Pull-off Strength of NPE-ZRP Coupons after Repair.....................137

Figure 5.8. Different Failure Modality of Pull-Off Strength for NPE-ZRP Coupons.....138

Figure 5.9. Condition of Sandblasted NPE-ZRP Samples after Outdoor Exposure........139

Figure 5.10. Hand Cleaned NPE-ZRP Samples after Outdoor Exposure........................140 
Figure 5.11. Coating Thickness of Outdoor Exposed NPE-ZRP Repaired Samples.......141

Figure 5.12. Pull-off Strength of NPE-ZRP Samples after Outdoor Exposure. ...............142

Figure 5.13. Condition of NPE-ZRP Scribed Samples after Cyclic Exposure................143

Figure 5.14. Condition of NPE-ZRP Unscribed Samples after Cyclic Exposure............144

Figure 5.15. Coating Thickness of NPE-ZRP Samples after Cyclic Exposure. ..............145

Figure 5.16. Coating Thickness of NPE-ZRP Samples after Cyclic Exposure. ..............146

Figure 5.17. Pull-off Strength of NPE-ZRP Coupons after Cyclic Exposure. ................147

Figure 5.18. OCP of Unscribed NPE-ZRP Samples during Cyclic Exposure.................149

Figure 5.19. OCP of Scribed NPE-ZRP Samples during Cyclic Exposure....................149

Figure 5.20. Icorr of Unscribed NPE-ZRP Samples during Cyclic Exposure................150

Figure 5.21. Icorr of Scribed NPE-ZRP Samples during Cyclic Exposure. ....................152

Figure 5.22. X-sectional Micrograph of Sandblasted Unscribed NPE-ZRP Samples.....153

Figure 5.23. Zinc Consumption of Sandblasted Unscribed NPE-ZRP samples..............154

Figure 5.24. Micrograph of Handcleaned Unscribed NPE-ZRP Samples.......................155

Figure 5.25. Micrograph of Sandblasted Scribed NPE-ZRP Samples..........................157

Figure 5.26. Micrograph of Sandblasted Scribed NPE-ZRP Samples...........................158

Figure 5.27. Repaired Coupons after Application of ZRP Coating..............................159

Figure 5.28. Coating Thickness of ZRP Coupons after Repair. ..................................160

Figure 5.29. Optical Micrograph of ZRP Coating Repair Coupon................................160

Figure 5.30. Coating Pull-off Strength of ZRP Coupons after Repair...........................161

Figure 5.31. Failure Modalities of Pull-off Strength Testing for ZRP Coupons.............162

Figure 5.32. Condition of the Sandblasted ZRP Samples after Outdoor Exposure.........163

Figure 5.33. Coating Thickness Change of ZRP Samples after Outdoor Exposure........164 
Figure 5.34. Pull-off Strength of ZRP Coupons after Outdoor Exposure. .165

Figure 5.35. Typical Condition of Scribed ZRP Samples after Cyclic Exposure. 166

Figure 5.36. Typical Condition of Unscribed ZRP Samples after Cyclic Exposure. ......166

Figure 5.37. Coating Thickness of ZRP Coupons after Cyclic Exposure. .167

Figure 5.38. Coating Thickness of ZRP Coupons after Cyclic Exposure. .168

Figure 5.39. Pull-off Strength of ZRP Coupons after Cyclic Exposure. .169

Figure 5.40. OCP of ZRP Samples during Cyclic Exposure. 171

Figure 5.41. $\mathrm{I}_{\text {corr }}$ of ZRP Samples During Cyclic Exposure. .171

Figure 5.42. Micrograph of Hand Cleaned Unscribed ZRP Samples. .173

Figure 5.43. Zinc Consumption of Sandblasted Unscribed ZRP samples. .174

Figure 5.44. Micrograph of Sandblasted Scribed ZRP Samples. .175

Figure 6.1. Contact Angle of the Pre-exposed Steel Substrate. 181

Figure 6.2. Optical Micrograph of NPE-ZRP Coating. 182

Figure 6.3. (A) Osmotic Blister formation on the salt contaminated samples

(B) Rust Development at the interface of Humidity exposed samples. .183

Figure 6.4. Different Failure Modality of Pull-off Strength. .184

Figure 6.5. Coating Pull-off Strength of ZRP (Test Group A). .185

Figure 6.6. Coating Pull-off Strength of NPE-ZRP (Test Group A). .186

Figure 6.7 Liquid droplet on Test Group A Sample in As-received Condition. .188

Figure 6.8. Percent Difference in Contact Angle for ZRP Samples (Test Group A). .....189

Figure 6.9. Percent Difference in Contact Angle of NPE-ZRP Samples (Group A).......190

Figure 6.10. Correlation between Contact Angle and Pull-off Strength (Group A).......191

Figure 6.11. Correlation between Surface Energy and Pull-off Strength (Group A)......192 
Figure 6.12. Liquid droplet on Test Group B Sample after Exposure. .195

Figure 7.1. OCP and Corrosion Current over Time. .205

Figure 7.2. EDS Point Mapping on NPE-ZRP Sample.

Figure 7.3. EDS Mapping of Salt-fog Exposed NPE-ZRP Unscribed sample (Top)......208

Figure 7.4. EDS Mapping of Salt-fog Exposed NPE-ZRP Unscribed sample (Botm)...209

Figure 7.5. EDS Mapping of a Salt-fog Exposed ZRP Unscribed sample. ...................210

Figure 7.6. EDS Mapping of a Solution Immersed NPE-ZRP Scribed sample..............211

Figure 7.7. EDS Mapping of a Solution Immersed ZRP Scribed sample.......................211 


\section{LIST OF SYMBOLS}

\begin{tabular}{|c|c|}
\hline $\mathrm{Zn}$ & Zinc \\
\hline $\mathrm{R}_{\mathrm{s}}$ & Solution resistance \\
\hline $\mathrm{R}_{\text {po }}$ & Coating pore resistance \\
\hline $\mathrm{C}_{\mathrm{c}}$ & Coating capacitance \\
\hline $\mathrm{C}_{\mathrm{dl}}$ & Double layer capacitance \\
\hline $\mathrm{R}_{\mathrm{p}}$ & Polarization resistance \\
\hline$\varepsilon_{0}$ & Vacuum permittivity \\
\hline$\varepsilon_{\mathrm{r}}$ & Relative permittivity \\
\hline A & Area \\
\hline $\mathrm{d}$ & Coating thickness \\
\hline$\rho$ & Resistivity \\
\hline $\mathrm{n}$ & Number of pores \\
\hline $\mathrm{Z}$ & Total impedance \\
\hline$\omega$ & Angular frequency \\
\hline$Y_{\mathrm{o}}$ & Pre-exponential term \\
\hline $\mathrm{I}_{\text {corr }}$ & Corrosion current \\
\hline$E$ & Potential \\
\hline
\end{tabular}




\section{ABBREVIATIONS AND ACRONYMS}

$\begin{array}{ll}\text { ATR } & \text { Activated Titanium Rod } \\ \text { ASTM } & \text { American Society for Testing and Materials } \\ \text { CPE } & \text { Constant Phase Element } \\ \text { EDS } & \text { Energy Dispersive Spectroscopy } \\ \text { EIS } & \text { Electrochemical Impedance Spectroscopy } \\ \text { FHWA } & \text { Federal Highway Association } \\ \text { HDG } & \text { Hot-dip Galvanizing } \\ \text { LPR } & \text { Linear Polarization Resistance } \\ \text { NACE } & \text { National Association of Corrosion Engineers } \\ \text { NPE-ZRP } & \text { Nano-Particle Enriched Zinc-Rich Primer } \\ \text { OCP } & \text { Open Circuit Potential } \\ \text { SSPC } & \text { Society for Protective Coatings } \\ \text { SEM } & \text { Scanning Electron Microscopy } \\ \text { SCE } & \text { Saturated Calomel Electrode } \\ \text { ZRP } & \text { Zinc-Rich Primer } \\ \text { Riffraction }\end{array}$




\section{CHAPTER 1 \\ INTRODUCTION}

\subsection{Background}

Corrosion is a major concern for the long-term durability and structural integrity of steel components of highway bridges. Approximately $15 \%$ of all bridges are structurally deficient due to corrosion (Materials Performance, 2002). Out of the approximate 600,000 highway bridges in the United States, 200,000 are steel bridges. Damage to the steel bridge superstructure can be exacerbated when it is unprotected or inadequately protected from the environment. In particular, aggressive marine environments that contain high concentrations of coastal airborne salt enhance corrosion. It was evident that corrosion of structural steel components was significant in the catastrophic collapse of the Silver Bridge (Point Pleasant, WV) in 1967 (Biezma1 and Schanack, 2007), the Mianus River Bridge (Connecticut) in 1983(NTSB, 1983), Lowe's Motor Speedway Bridge (North Carolina) in 2000 (Cederquist, 2000), Kinzua Bridge (Pennsylvania) in 2003 (Jeffery, 2009), and Leo Frigo Memorial Bridge (Wisconsin) in 2013 (Khalid et.al., 2018).

The application of protective coatings has been widely used for corrosion mitigation of atmospherically exposed structural steel. Different coating systems for corrosion protection of steel bridges have been developed and implemented over time due to the changes in environmental and health regulations, economics, and advances in technology. Even with the continuous development of coating technologies, coating systems are still susceptible to deterioration and thus unable to provide protection for the long-term design bridge service life. Periodic maintenance of coatings is required for 
additional service life against exposure to its surrounding environment. Furthermore, maintenance including coating removal, containment, and application is costly. Of the estimated $\$ 8.3$ billion annual costs of corrosion in highway bridges, $\$ 500$ million is expended only for coating maintenance of highway steel bridges. So, effective and costefficient coating systems that meet or exceed health and environmental regulations are always of interest.

The majority of the steel bridges in the interstate highway system were constructed between the 1950s and 1980s. Until the 1970s, bridges were generally coated by alkyd-based paint containing toxic lead and chromate (Kogler Jr. and Chong, 1997). Approximately 80 to 90 percent of the 200,000 steel bridges in the United States were coated with lead or other toxic heavy metal-based inhibitor coatings (Myers et al., 2010). These old technology coating systems usually consisted of several layers and required costly routine levels as well as major paint maintenance within eight to ten years of service life. Many of those coating systems became prohibited by Environmental Protection Agency regulations due to environmental and health hazards. After the 1970s, an entirely different coating technology containing sacrificial zinc pigments was introduced for bridge application; and at present, the zinc-rich primer based three-coat system is widely used in the United States. The metallic zinc pigments ideally would provide corrosion resistance by sacrificial protection. The three-coat system typically consists of either an organic or inorganic zinc-rich primer (although other primers have been formulated) followed by an epoxy midcoat and a topcoat. Generally, the performance of zinc-rich paints outperformed the previous lead-based paints. Nevertheless, the life of the coating is at best only half of the required design life of the 
bridge. Furthermore, premature degradation may occur if there are flaws in the system due to improper application.

Repair of the degraded coating material should not be overly complicated and must be cost-effective. Appropriate surface preparation and identification of environmental exposure parameters such as humidity, surface moisture, air-born salt contamination and their effects on coating physical properties and corrosion mitigation should be considered. It was reported that modern paint coatings may require early maintenance especially if exposed in aggressive environments (Florida Department of Transportation (FDOT) Bridge Work Plan, Personal Communication, June 21, 2018). The long-term effectiveness of coating systems is of major importance to reduce maintenance costs. The coating system not only should provide adequate corrosion control and meet environmental and health regulations, but also the coating durability should be attuned to expected bridge design life. The selection of compatible repair coating material and the corresponding level of surface preparation is the critical parameter for proper protection from its exposure environment. In consideration of ease of coating application, application quality, costs, and durability in aggressive exposure conditions, new materials for corrosion mitigation are needed. Many coating systems have been introduced by the industry and government sectors for varying applications. These commercially-available materials may have a useful application for highway bridges. Novel coatings containing carbon nano-particles with the zinc-rich primer (NPEZRP) have garnered attention for possible highway bridge applications as promoted for providing better electrical continuity of the zinc pigment for enhanced cathodic protection for long service life. Coating durability, robustness, and repair considerations 
in aggressive environments relevant to highway bridges should be evaluated. Topics of importance include identifying appropriate surface preparation, application, compatibility and determining resiliency to environmental exposure conditions during and after repair.

\subsection{Problem Statement and Research Objective}

The need for effective corrosion mitigating coating systems with longer service life and reduced maintenance requirements are important to maintain the civil highway infrastructure in the U.S. Commercially available coating systems specified for bridge applications do not necessarily have service life commensurate with bridge service specification and require regular inspection and maintenance. Other limitations for available coating systems are application and repair susceptibilities. Therefore, there is continued interest to explore alternative novel coating systems that may have a suitable application for highway steel bridges. A nano-particle enriched zinc-rich primer (NPEZRP) based coating system was identified for study due to its possible beneficial characteristics. Application and exposure conditions of interest include coatings for structural steel. The first step to evaluate any material is to identify possible degradation modalities to assess the long-term exposure durability. In marine bridge, structural steel application, important environmental and exposure conditions include alternate wet/dry cycle, moisture availability, temperature, humidity, salt exposure, ultraviolet exposure, $\mathrm{pH}$, crevice environment and localized coating defects. The environmental and exposure conditions, as well as modality and severity of initial coating defects, can all contribute to the degradation of the coating and its efficacy as a corrosion mitigation system. Preventative regular bridge maintenance reduces the risk of catastrophic failure and can be beneficial for the economy by providing extended service life. For steel bridge, paint 
maintenance selection of compatible repair material and appropriate application is crucial for corrosion durability. Like a new coating application, harsh environmental exposure and contaminants during as well as after the coating application may also be detrimental for repair coating durability. The application of the repair coating should not be overly complicated or time-consuming. Minimizing the impact on transportation is important. Outdoor and lab testing was intended to elucidate coating degradation. The research goals include identifying and predicting coating durability and the ability to mitigate corrosion. So, the objective of this study was to identify the corrosion mitigation and degradation mechanism of NPE-ZRP coating system, also the robustness of the coating system to use for repair application in marine exposure relevant to highway bridge structural steel elements.

\section{Hypothesis}

Zinc-rich primer-based coating system containing carbon nano-particles will provide improved coating durability for marine steel bridge applications by providing enhanced mechanical (cohesion and adhesion) and corrosion protection properties. The nano-particles will provide extended galvanic coupling of the sacrificial zinc pigments with the exposed steel substrate and thus extend the life of the steel structure. Coating application in adverse environmental conditions will not cause an additional detrimental effect on durability due to the presence of carbon nano-particles.

\section{$\underline{\text { Research Objectives and Questions }}$}

The objective of the work presented here was to verify that if NPE-ZRP coating can provide enhanced coupling of the zinc pigments with the steel substrate. Testing included evaluation in extended outdoor atmospheric and salt-fog exposures. Since 
moisture and salt were expected to be major factors in marine atmospheric exposure, a set of testing was made by immersing coated steel samples in $3.5 \mathrm{wt} \% \mathrm{NaCl}$ solution. Testing here considered localized coating damage that exposed the steel substrate. Furthermore, it was of interest to identify the influence of nano-particles polarization behavior of embedded zinc pigments. Test conditions to promote the electrochemical activity of the zinc (including both oxidation and reduction reactions) included various levels of electrochemical polarization. In order to assess the effect of nano-particles on zinc electrochemical coupling and steel corrosion development, the extent of zinc consumption as well as coating degradation was addressed. A traditional zinc-rich primer (ZRP) was studied to reference the performance of NPE-ZRP. To meet the research objectives the following questions needed to be addressed:

1. How does the nano-particle enhance the coating durability?

- Discriminate extrinsic parameters (such as coating thickness, zinc pigment distribution, etc) of a commercially available ZRP and NPE-ZRP coating that can affect coating durability.

- Determine the extent of corrosion mitigation afforded by NPE-ZRP compared to conventional ZRP in different environments containing variable moisture and salt content.

- Identify if nano-particle presence can promote effective galvanic coupling of the reaction sites away from the defect. Identify if beneficial cathodic polarization can promote extended zinc anodes with time. Identify if promoted galvanic coupling can reassure extended cathode. 
- Identify mitigation of mechanical coating degradation (such as adhesion loss, disbondment and anodic blistering) afforded by the enhanced tensile property of NPE-ZRP coating.

- Identify an approach to estimate the long-term performance of NPE-ZRP coating.

2. What are the negative effects of adverse surface conditions on NPE-ZRP coating durability?

- Determine the effects of surface contamination on undercoating adhesion, electrical continuity of the pigments to the steel and corrosion development.

- Determine coating integrity in aggressive exposure conditions.

3. What are the effects of adverse environmental exposure on NPE-ZRP coating durability for repair applications?

- Determine the effects of inappropriate surface preparation and adverse contaminants on undercoating adhesion, electrical continuity of the pigments to the steel and corrosion development.

- Determine the effects of adverse environmental exposure on the integrity of repair coating durability.

4. What is the influence of nano-particles on the major interdependent coating parameters related to coating durability?

- Develop a statistical model that can correlate the coating parameters with coating durability.

- Identify the effect nano-particles on coating performance. 


\subsection{Research Approach}

The proposed research approach includes:

1. Characterize physical, metallurgical, and other material traits of a NPE-ZRP (commercially available) as well as a conventional ZRP coating system used in current practice. Identify the modality of coating defects formed during application as well as coating resilience to surface damage.

2. Expose coated samples with and without intentional defects to outdoor exposure (at beach site and inland locations), in laboratory exposure with aqueous solutions representative of pooled runoff water and aggressive salt-fog environments to assess the coating integrity for long-term corrosion durability.

3. Exposure to different levels of polarization with time, to identify the effect of electrochemical interaction of zinc pigments with steel defects to mitigate corrosion with the presence of nano-particles.

4. Assess corrosion development and efficacy of nano-particle presence on efficient galvanic protection by applied electrochemical measurements (OCP, LPR, EIS, and potentiostatic polarization).

5. Test sample preparations with conditions representative of conventional repair application.

6. After diverse surface preparation and adverse environmental pre-coating exposure, expose coated samples with and without intentional defects to aggressive accelerated cyclic testing (include immersion in aqueous solution with $\mathrm{Cl}^{-}$, dry exposure in low humidity and aggressive salt-fog exposure) to identify the effect of alternate wet and dry exposure on coating robustness to repair susceptibility. 
7. Material evaluation techniques such as visual, physical (thickness, pull-off strength), metallurgical assessment (optical and electron microscopy, Image Processing, EDS and XRD), to elucidate the findings from entire test exposure.

8. Develop a statistical model by correlating the coating durability parameters to identify the influence of nano-particles.

\subsection{Organization of the Dissertation}

This dissertation is organized as follows:

Chapter 2 represents the present state of knowledge on the current highway steel bridge protection practice through the application of the protective coating and general overview of the basic concept of electrochemistry related to coating evaluation.

Chapter 3 represents the methodology to achieve the objective of the research. The detail test procedures and evaluation technique of the test samples are documented in this chapter.

Chapter 4 presents the results of the tested samples for the application of NPEZRP coating for new structural steel, which was exposed to different test exposure. Associated discussion is made based on the test results to describe the degradation process related to that environment.

Chapter 5 presents the results of the tested samples for the application of NPEZRP coating for repair application, which incorporated deficient surface preparation and were exposed to different test exposure. Associated discussion is made based on the test results to describe the degradation process related to that environment.

Chapter 6 presents the assessment of the effect of carbon nano-particles on the durability of NPE-ZRP coating. How the addition of nano-particles can enhance the 
performance of the NPE-ZRP coating system is explored by surface measurements and statistical model.

Chapter 7 summarizes the findings from the outdoor, accelerated salt-fog and electrochemical tests that demonstrate the role of nano-particles on the zinc consumption mechanism of NPE-ZRP coating system.

Chapter 8 summarizes the conclusion about the NPE-ZRP coating durability in exposure related to aggressive marine bridge environment and future recommendation.

Some content in this dissertation has been published in report form to the sponsoring agency (Saiada, Sabbir and Lau, 2019) and published in conference proceedings (Saiada et al, 2017-19). Those published contents have been in part reproduced here. 


\section{CHAPTER 2}

\section{LITERATURE REVIEW}

\subsection{Steel Bridges and Service Environment}

In the US, there are approximately 200,000 highway steel bridges. These bridges are located over a variety of exposure environments in terms of temperature, humidity, rainfall, ultraviolet radiation from the sun, pollutants, and airborne salts. Generally, the bridge environment is classified based on the potential threats from its surroundings as,

- Mild (rural): Little to no exposure to natural airborne or deicing salts, industrial pollutants, low humidity, and rainfall, usually located in an inland location.

- Moderate (industrial): Exposed to some or occasional airborne or deicing salt runoff, corrosive industrial contaminants, moderate to high humidity, usually located in a heavily polluted urban area.

- Severe (marine): Exposure to high airborne salt or deicing salt, high humidity and moisture, usually located in proximity to the coastal area.

Table 2.1 shows the rate of carbon steel corrosion with exposure variation. The durability of any corrosion protection system for structural steel vastly depends on its surrounding environments. So proper corrosion protection technologies based on the surrounding exposure environment are required for the long-term durability of the structural systems. 
Table 2.1. Corrosion Rates of Carbon Steel in Different Environmental Exposure (American Galvanizers Association, 2019).

\begin{tabular}{cc}
\hline Atmosphere & Corrosion Rate $(\boldsymbol{\mu m} / \mathbf{y e a r})$ \\
\hline Rural & $4-60$ \\
\hline Urban & $30-70$ \\
\hline Industrial & $40-160$ \\
\hline Marine & $60-170$ \\
\hline
\end{tabular}

\subsection{Coatings for Corrosion of Steel Bridges}

Coatings are the most widely used technology to protect steel infrastructure against its service environment and have been developed over the years to extend the life of the structure by improving the corrosion resistance. Protective coating systems are generally divided into three broad categories based on their mechanisms: barrier formed between the substrate and environments, inhibition of the corrosion processes, and coating acting as sacrificial materials. Barrier protection is the mode of protection provided by intact coatings whereas the other two modes are designated as active protection of damaged areas by coating components (Bierwagen, 1996).

\subsubsection{Barrier Coating}

A barrier coating creates an insulating and physical barrier, thus reducing the passage of corrosive elements and the availability of moisture through the coating layer to the substrate. The effectiveness provided by a barrier coating system highly depends on its permeability as well as coating thickness and binder type (Sørensen et al., 2009). The low conductivity of the electrolyte at the metal coating interface minimizes the transport of corrosion current between the anode and cathode, (Hare, 1989). The 
properties of the coating/metal substrate interface are closely tied to the barrier properties

of the system as failure was reported due to lack of adhesion (Bierwagen, 1996).

\subsubsection{Inhibitive Coating}

Inhibitive coating develops a passivation layer by the reaction of inhibitive pigments with the steel substrate in presence of moisture, which prevents access of the corrosive substance to the substrate (Amo et al., 2002). The efficiency of the inhibitive coating depends on the balance between the barrier properties of the coating and the degree of permeability to permit the diffusion of water to activate the pigments (Liu, 1998). The inhibitive pigments are generally water-soluble inorganic salts which facilitate their transportation to the defect site (Sørensen et al., 2009). The associated problem is that if the solubility is too high, blistering can form (Prosek and Thierry, 2004).

\subsubsection{Sacrificial Coating}

Sacrificial coatings are developed based on the principle of galvanic corrosion. The substrate is protected by a metal or alloy that is electrochemically more active than the material to be protected. A more active metal than steel becomes an anode when in direct contact with the less active steel substrate and eventually protect the steel substrate from corrosion. Sacrificial coatings are applied as primers. The effectiveness of the coating depends on the electrical contact of the substrate with the sacrificial metal as well as on the transfer of the galvanic current (Sørensen et al., 2009). Thus, sacrificial coatings should be highly pigmented to ensure proper metallic contact between the individual particles of the sacrificial metal. Coatings systems can also exhibit multiple corrosion mitigation characteristics. For example, HDG and Metalizing is a barrier but can have 
sacrificial properties depending on environmental conditions. The zinc-rich primer system serves as a sacrificial coating but has barrier characteristics also (FHWA publication 1995).

\subsection{Present Practice of Bridge Coating System}

Prior to 1965, bridge coatings were generally oil-based multi-layers (5-6) coating systems containing lead/ chromium pigments as corrosion inhibitors. These coating systems were usually applied directly to the steel substrate after power tool cleaning (SSPC-SP3) covered with mill scale. Some level of maintenance was required typically within eight to ten years of application and another coating layer was added as repair policy (FDOT Bridge Work Plan, Personal Communication, June 21, 2018). As a result, several layers were added on with time and subsequently adhesive failures were reported between the coating layers and from the steel substrate even sometimes because of the weight of the paint layers. Environmental regulations and advancement in paint chemistry have driven continuous changes in paint formulation. With the development of abrasive blasting technology to remove mill scale that provides a clean surface for paint application, the coating industry shifted from several layered lead-alkyd paint systems to a new generation high performing zinc enriched coating system that provides galvanic protection. With the modern development and advancement in technology, the coating lifetimes to first major maintenance have gradually increased from 12 and 15 years to 20 and 25 years (NPL Corrosion Guide, 2015). Some coating systems that are currently used are described next.

\subsubsection{Paint Coating System (Zinc-Rich Three-coat system)}

Paint coating systems are the most commonly used material to protect steel 
bridges. Previous multi-coat systems have been replaced mostly by three-coat systems, and research is still going on to improve its performance.

The majority of state highway departments specified the zinc-rich three-coat systems as a conventional coating system for highway steel bridges due to numerous technical, political, and economic issues (FHWA publication 2015). The protection mechanism of the zinc-rich primer (ZRP) coating system is based on the galvanic coupling of the zinc pigments referred to as cathodic protection as well as barrier protection of the coating layers. The galvanic feature differentiates ZRP from traditional barrier coatings. Zinc-rich primers are usually inorganic zinc (IOZ) or organic zinc (OZ). IOZ primers consist of zinc metal pigment mixed into an inorganic silicate-based paint binder. This binder can be either solvent-borne (ethyl silicate) or waterborne (alkali silicate). OZ primers contain zinc metal pigment mixed into an organic paint resin such as epoxy or urethane. The current "gold standard" for steel bridge coating practice involves the use of a three-coat system consisting of an inorganic zinc-rich primer, an epoxy midcoat, and a urethane topcoat (Figure 2.1). The different layers of three-coat systems have specific functions. The primer provides sacrificial protection and the midcoat (usually epoxy binders) acts as an additional barrier to the ingress of aggressive environment agents (moisture and chemicals) toward the steel/coating interface. Corrosive agents are transported to the primer/midcoat interface by diffusion through the above layers (Kolek, 1997). Usually, three types of epoxy coatings are used as an intermediate layer such as epoxy ester, epoxy lacquer, and a two-component epoxy. Epoxy ester is a vegetable oil-modified resin which is alkali resistance and epoxy lacquer consists of high molecular weight that needs short curing time. Two-component epoxies 
are polyamides, comprises of greater flexibility and longer pot life (Chang and Chung, 1999). Usually, these epoxy intermediate layers provide excellent resistance to corrosive agents. Topcoats are used to retain coating aesthetics and to provide wear \& tear as well as UV resistance. Typically, urethane and polyurethane binders are used as oil-modified urethane, moisture-cured urethane, and two-component urethane. Oil modified pigmented urethanes are not appropriate for steel infrastructure due to a lack of durability. Moisture cured urethane use the ambient moisture for curing and pigmentation is not suitable due to moisture susceptibility thus only used as a clear finish. Two-component urethane use polyols, polyethers, polyesters or acrylics with urethane to produce a resistant and durable coating. For three-coat systems, hydroxylated acrylic or hydroxylated polyester binded urethanes are the most commonly used topcoat due to their superior UV resistance and faster drying capacity (Chang et al., 1999).

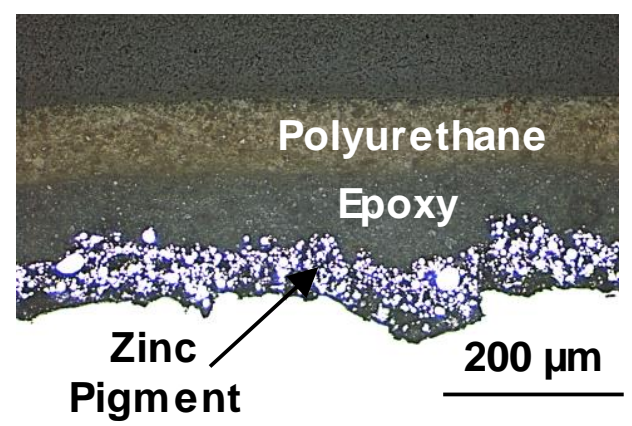

Figure 2.1. Metallographic Cross-section of Three-coat System.

ZRP ideally prevents corrosion of the steel at small coating damage sites and coating film holidays by sacrificing the zinc pigments well known as cathodic protection. Proper galvanic action depends on the chemical nature of the binder, amount of metallic zinc known as pigment volume concentration, the grain size of the zinc pigments, zinc tosteel area ratio and the coating film thickness (Hammouda et al., 2011). The electrical 
continuity between the zinc pigments and the steel substrate is the crucial parameter for the effectiveness of zinc pigments to provide efficient galvanic protection (Abreu et al., 1999). For efficient electrical continuity, the pigment volume concentration (PVC) of the zinc pigments in the coating should exceed the critical PVC. It has been reported that the quantity of zinc pigments (even with content as high as $80-90 \%$ wt) alone cannot ensure effective electrical continuity to provide long-term galvanic protection (Shreepathi, et. Al., 2010). Studies also have found that zinc particle shape is also critical for continuity and spherical pigments cannot provide efficient electrical contact (Schaefer and Miszczyk, 2013). Furthermore, coatings with high pigment content can be brittle as well as porous and exhibit poor substrate and/or inter-coat adhesion. It also may create difficulties in application due to high viscosity and poor dispersion (Park and Shon, 2014). After the consumption of connected zinc pigments, the long-term protection can also be in part due to the barrier protection provided by the zinc oxidation products. The barrier mechanism develops from the blockage of inherent coating pores by zinc corrosion products. The formation of zinc oxidation product blocks the coating porosity and leads to a highly compact structure that can hinder the ingress of adverse corrosive agents to the steel substrate. But the zinc consumption for cathodic protection can also affect electrical continuity by creating hindrance to electron flow. Thus, electrical continuity and coating porosity, define the anticorrosive properties of ZRP.

The corrosion protection of the three-coat system with inorganic zinc primers was reported to be better for new construction than with organic zinc primers. However, the sensitivity of inorganic zinc primers to surface conditions limits its application to controlled settings in the shop. Research on three-coat systems by the Michigan 
Department of Transportation concluded good early performance to protect the steel from corrosion after five years (Phifer, 1993). The Wind Gap Bridge near Pittsburgh, the Martin Luther King Bridge in Richmond and MoDOT bridge A2107 in Franklin are some examples of bridges coated with inorganic zinc-rich primer with records of long-term durability. When the bridges were evaluated after $\sim 20$ years, the coating was found to be in excellent condition with only a few areas with slight coating degradation in need of touch-up attention (Kline, 2009). FHWA initiated a research program in August 2009 to identify coating systems that can provide long-term durability with minimal maintenance. Eight selected coating systems with a promising performance in part based on prior experimental data from accelerated laboratory testing and outdoor exposure testing were evaluated (Kodumuri \& Lee, 2012). The evaluation consisted of accelerated laboratory testing (consisting of cyclic environmental exposure to temperature, $\mathrm{UV}$, and moisture, and salt) and outdoor marine and simulated salt exposure environments. The study concluded that the three-coat systems with zinc-rich epoxy and polyurethane topcoats performed well but none of the coating systems can meet long-term maintenance-free coating applications.

\subsubsection{Hot-dip Galvanizing}

Hot-dip galvanizing (HDG) is a method of applying metallic coatings to structural steel and has been used to coat bridge components for many years (Zhmurkin, 2009). Hot-dip galvanizing involves immersing the steel components in a bath of molten zinc. The immersed surface form an integral bond by developing a thick zinc-iron alloy coating with different alloy composition (Figure 2.2) defined as Gamma, Delta, Zeta, and Eta layer (American Galvanizers Association, 2017). The thickness of the galvanized 
coating is influenced by the size and thickness of the workpiece, the steel surface preparation, and the chemical composition of the steel. The galvanized coating protects the steel by providing an impervious barrier that does not allow moisture to contact the steel. This barrier layer develops by forming zinc corrosion products when exposed to the atmosphere. To prolong the service life of galvanized surface an additional barrier layer of zinc coating can be introduced as a duplex system (American Galvanizers Association, 2012). The combination of galvanized steel and painting can provide enhanced corrosion protection, but paint delamination due to weak bonding between paint and the metallic substrate can reduce the durability of duplex systems (Cabanelas, et. al.2007).

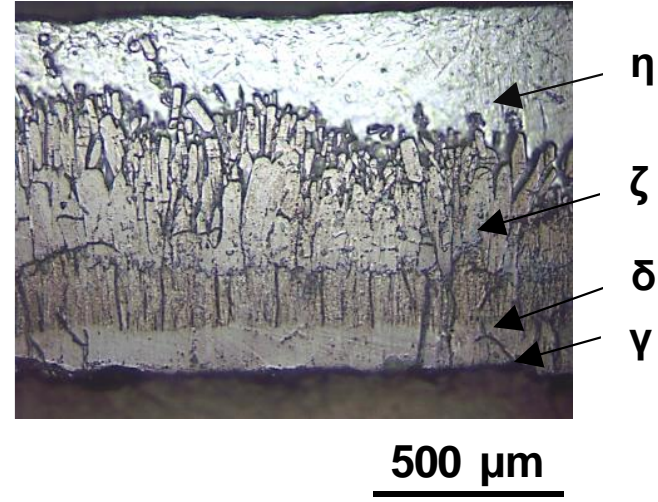

Figure 2.2. Metallographic Cross-section of Hot-dip Galvanizing (Sabbir, 2017).

Many transportation departments have adopted hot-dip galvanizing due to its performance but transportation costs, as well as galvanizing kettle size and availability, have been an important factor (FHWA Publication, 2015). The formation of the alloyed layer depends on the steel chemistry and the processing condition. All the layers may not be formed depending on these conditions (Yeomans, 2004). Furthermore, hydrogen embrittlement due to the accommodation of hydrogen at the time of surface cleaning prior to HDG is another negative aspect. According to the American Galvanizing 
Association, hot-dipped galvanized items will last 75 to 100 years in an aggressive marine environment (American Galvanizers Association, 2012). However, documentation of early age peeling or delaminating of galvanizing and rusting of steel substrate has been made (Helsel, 2015).

\subsubsection{Metallizing}

Metallizing consists of the thermal spraying of zinc (Figure 2.3), aluminum or zinc-aluminum alloy directly onto steel surfaces (Bernecki et al., 1997, Koger et al., 1998 \& Chang et al., 1999). The molten metals as a wire or in powder form are applied using an airstream spray onto the steel surface in a thin film. Metallizing can be applied in the shop or in the field with a specialized instrument. The steel surface is prepared by grit blistering or chemical etching for proper mechanical bonding. Aluminum requires more surface roughness than zinc (Chang and Georgy, 1999). Surface preparation specifications include SSPC-SP 5/ NACE-1 (White metal blast cleaning), SSPC-SP 10 / NACE-2 Near white metal blast cleaning, (Chang and Georgy, 1999). Metalized coatings shield the steel surface by both sacrificial and barrier protection. The coating provides barrier protection, especially when applied along with a topcoat, whereas zinc or aluminum in the coating protects the steel at the location of any damage (Kogler Jr. and Chong, 1997). Sealers such as acrylic urethane, polyester urethanes, vinyls, phenolics, epoxy or thermal sprayed polymer can be used to enhance service life by sealing the pores in the coating (Chang and Georgy, 1999). 


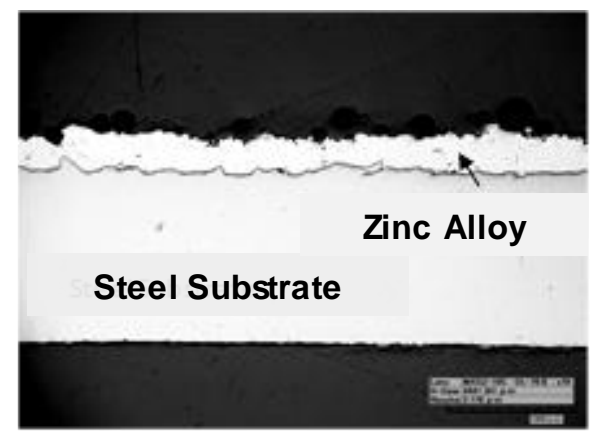

Figure 2.3. Thermal Spray Coating (Figure by Lau \& courtesy of FDOT).

The cost of metalizing is high compared to traditional paint systems, but many transportation departments have adopted metallization due to its performance. Thermal spray coatings are susceptible to degradation on substrates with poor surface preparation (Chang and Georgy, 1999) which may limit their efficiency for field application. Localized corrosion was observed in the early age of metalized coating of a bridge in Connecticut due to improper surface preparation.

\subsection{FDOT Steel Bridge Performance}

\subsubsection{Steel Bridges of Florida}

The FDOT bridge inventory includes 1,206 steel bridges (Data provided by FDOT). These bridges are located in varying service environments. In Florida, 94 steel bridges are located in coastal regions, 627 bridges are located over inland locations and the remaining 468 bridges are located over the water body (Figure 2.4). A significant number of these bridges are located in major population centers supporting vital transportation infrastructure especially in coastal regions and most of the coastal bridges are movable bascule bridges. FDOT owns a large population of the movable bridges in the U.S. (Catbas, 2013). Figure 2.5 shows the age distribution of $~ 1200$ of those Florida steel bridges. It is evident that the bridge inventory has mostly bridges with relatively 
short service times. Sixty percent of the steel bridges are under 30 years and $40 \%$ of them are under 20 years of service life.

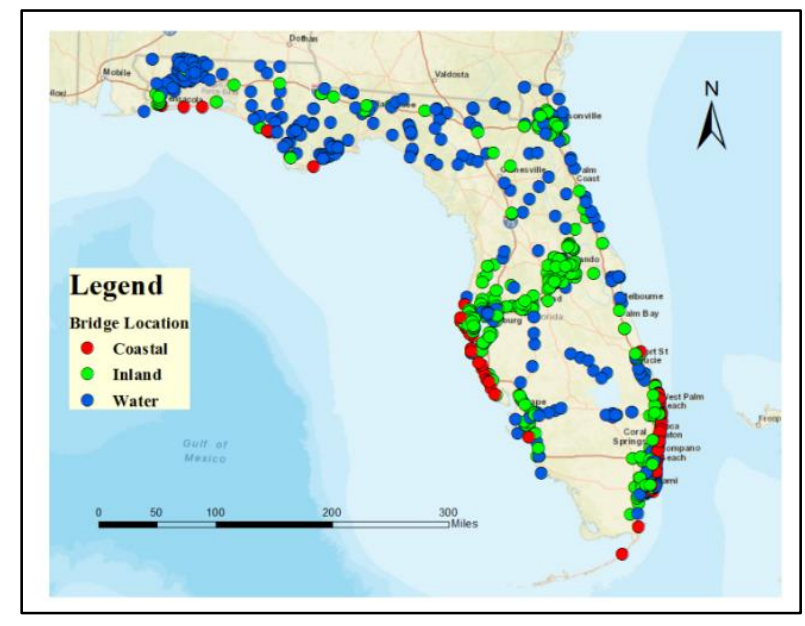

Figure 2.4. Location of Florida Steel Bridges.
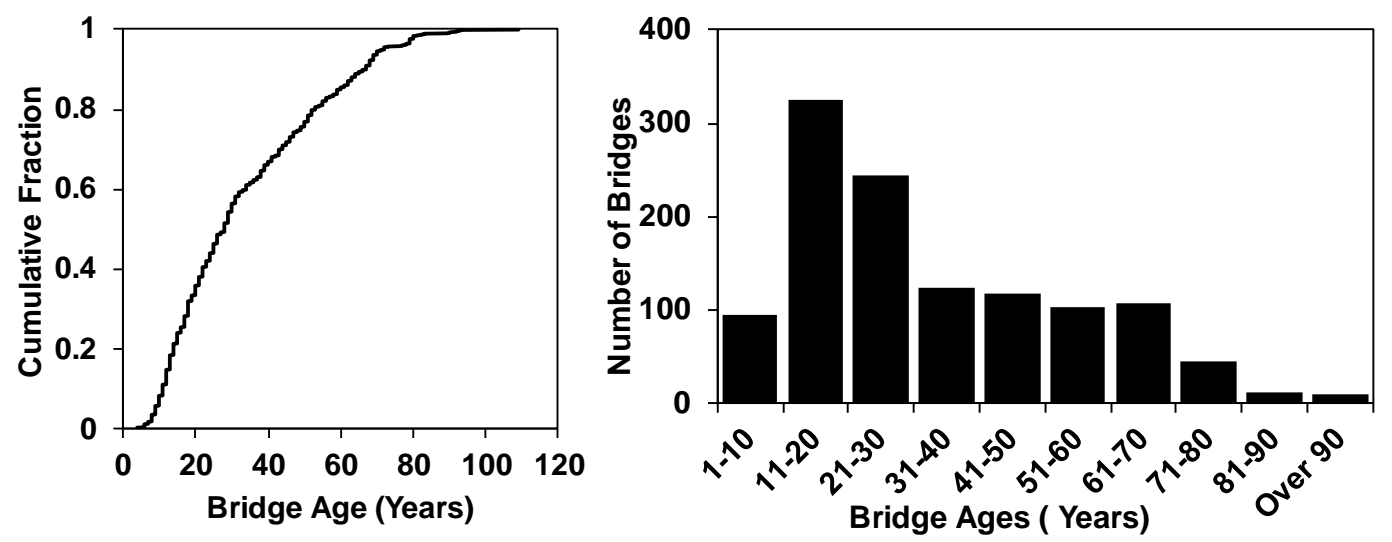

Figure 2.5. Age Distribution of Florida Steel Bridges.

\subsubsection{FDOT Bridge Coating Performance}

Florida started using the three-coat paint system for new structural steel in the 1980s with a projected service life of more than 30 years. The coating systems of old bridges were also replaced by the three-coat system due to environmental regulation and advancement in coating technology. Records from inspections of painted steel bridges (Data provided by FDOT) in FDOT Districts indicated that it is possible early 
deterioration of the coating system within $\sim 15$ years. Although no severe coating deterioration was observed up to $\sim 15$ years, the indication of degradation of coating elements would suggest that the onset of damage may occur in the near future. FDOT bridge work plan (Data provided by FDOT) details and inspection records are provided below.

Inspections reported that there is a possible early coating degradation within $\sim 15$ years after the initial application (Data provided by FDOT). Usually, spot or zone repair recommended to stop further degradation. Within a short period of time, the repair started deteriorating again and gets worse with environmental exposure. The average year for the repainting of the steel bridges is around 25 years after the paint application (Figure 2.6).

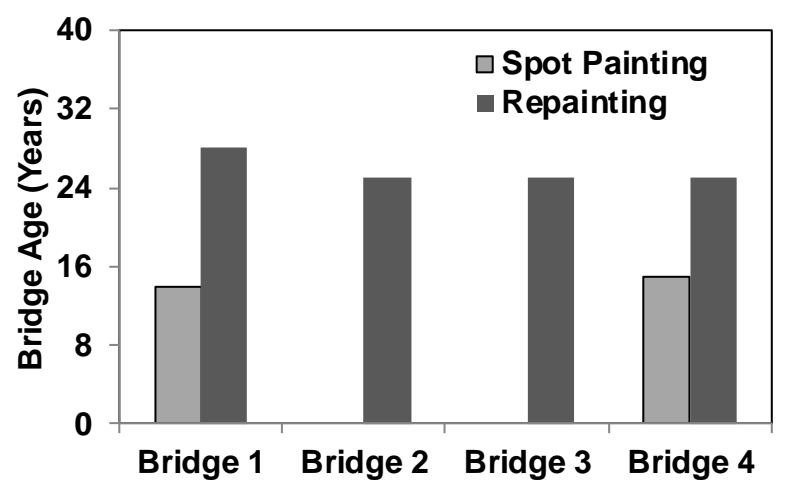

Figure 2.6. Age for Bridge Paint Repair (FDOT Bridge Inspection Report).

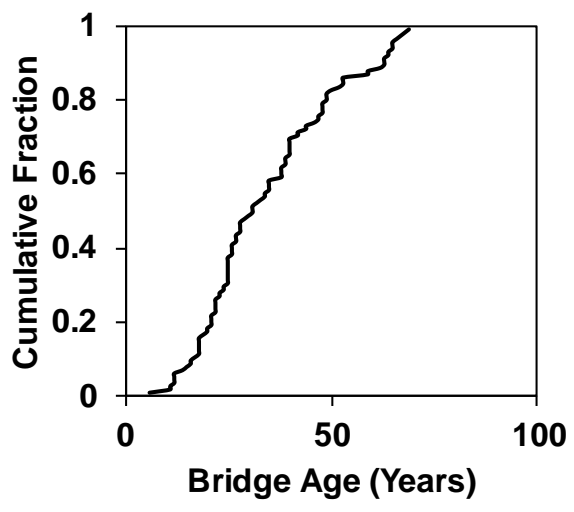

Figure 2.7. Age Distribution of Repainted Bridges (FDOT Bridge Work Plan 08-18). 
Figure 2.7 shows the age distribution of $\sim 500$ steel bridges from the Bridge Work Plan 2008-2018. It is evident that more $50 \%$ of the repainted bridges were less than 20 years of service life. The bridges over 30 years of service life $(>35 \%$ of the repainted bridges) can have multiple paint repair as there was no detail information available about the paint system in the survey report.

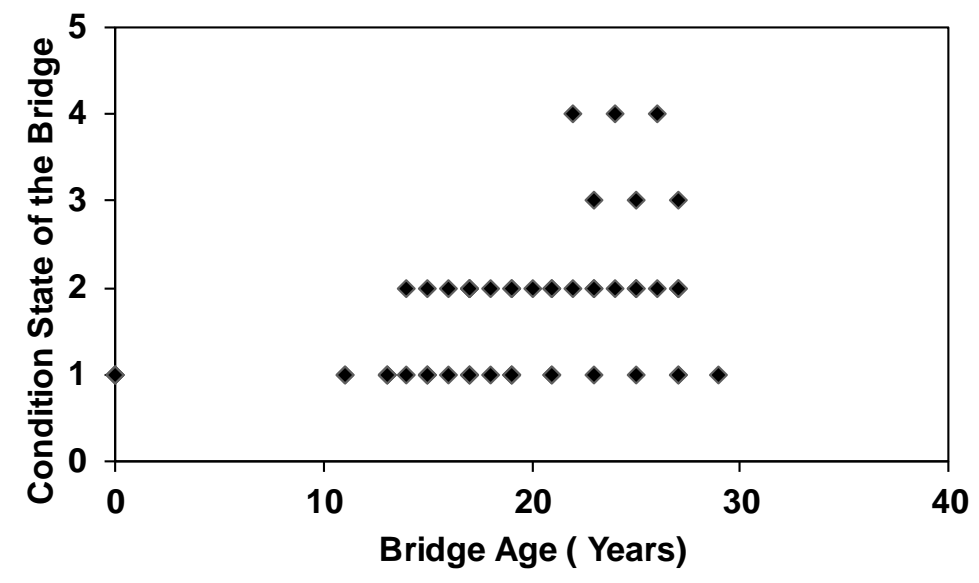

Figure 2.8. Bridge Condition State over Age (FDOT Bridge Inspection Report).

Figure 2.8 shows the condition state of different bridges over the service life. As can be seen, bridge coating started to deteriorate and changed from condition state 2 to 1 , typically after 14 years. Severe coating degradation (condition state 3/4) was observed after service of 22 years. Figure 2.9 illustrates the length of bridge condition state over the service life of four different bridges located over inland and water body. The typical recommendation for spot painting was reported at the age of $\sim 15$ years. After initial spotpainting, a full repair was recommended when a significant portion of the bridge turned to condition state $2 / 3$ typically at the age of $\sim 25$ years. 

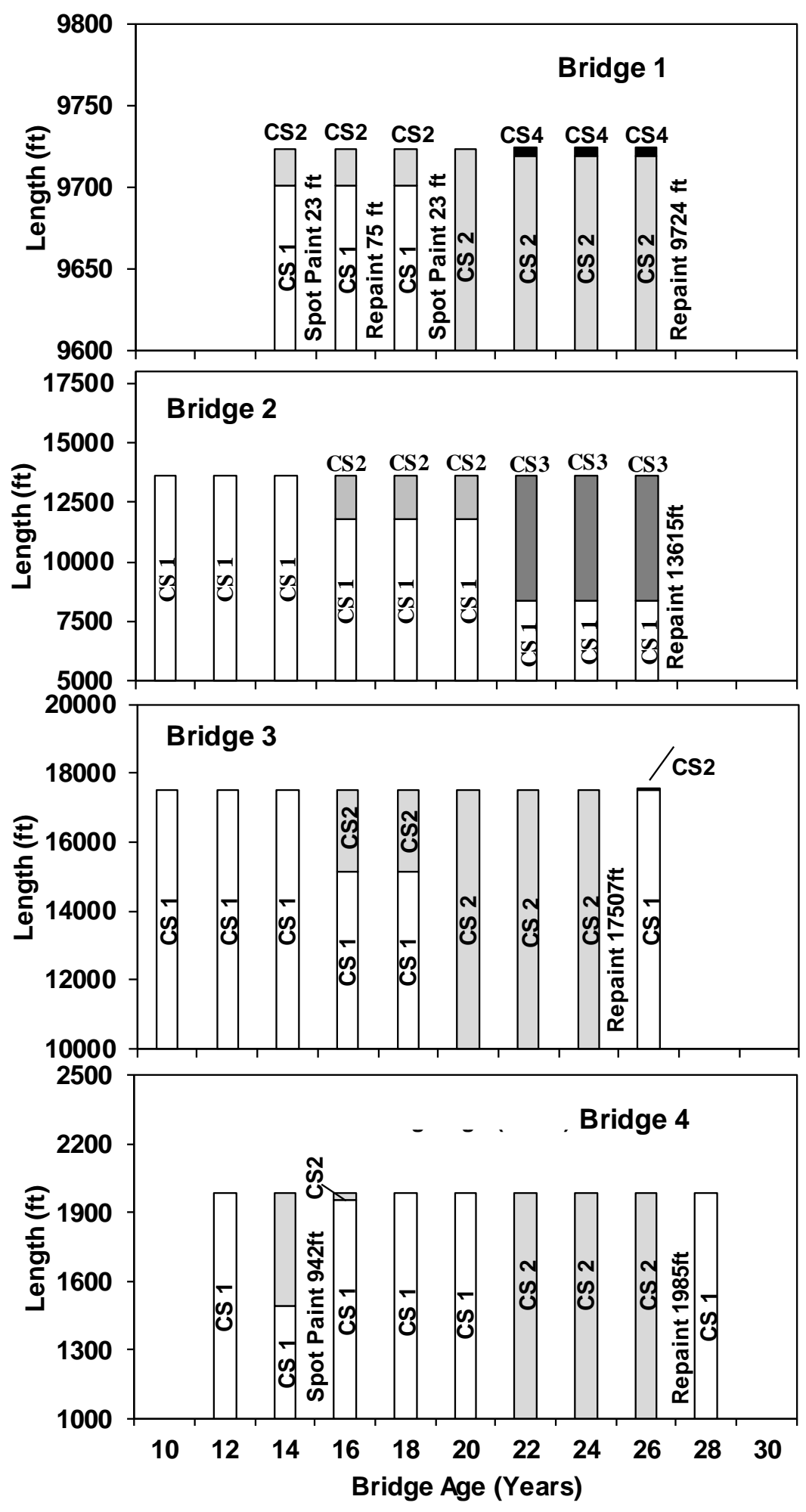

Figure 2.9. Condition State over the Service Life (FDOT Bridge Inspection Report). 


\subsubsection{FDOT Bridge Coating Maintenance Cost}

Most of the Florida steel bridges contain ZRP coating systems as they are newly constructed or having paint replacement from previous lead and chromate-based paint systems. Field performance history showed that the steel bridges often require repaint long before their expected service life period. According to FDOT Bridge Work Plan reports from 2008 to 2018 (Data provided by FDOT), the replacement of steel bridge paint systems typically consists of a significant part of the total repair plan (Figure 2.10). For each of these years, the replacement of the bridge coating system was the highest number of individual repair work, as shown in Figure 2.11. Furthermore, as shown in Figure 2.12, a general growing trend in the amount of paint system replacements is observed. The cost of the associated maintenance requirements for paint coating systems is shown in Figure 2.12 and the annual costs typically exceed \$25 million (Pouliotte 2012, Clarke 2016).

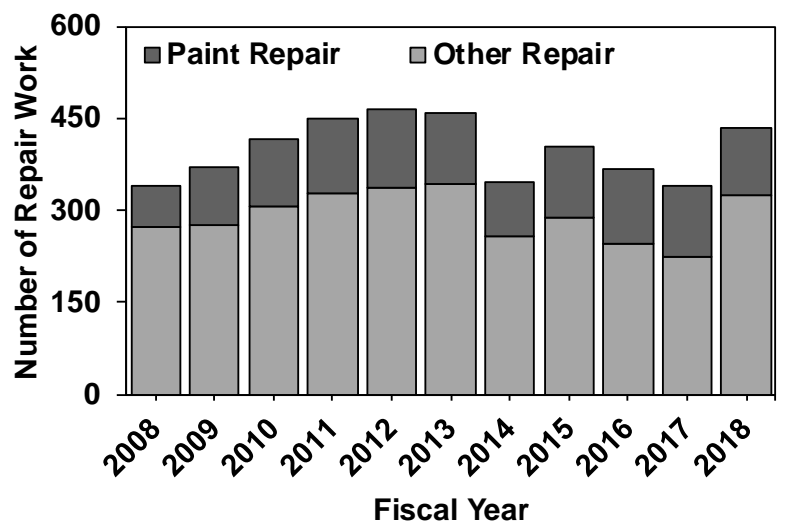

Figure 2.10. The number of Repainted Bridges (FDOT Bridge Work Plan 08-18). 


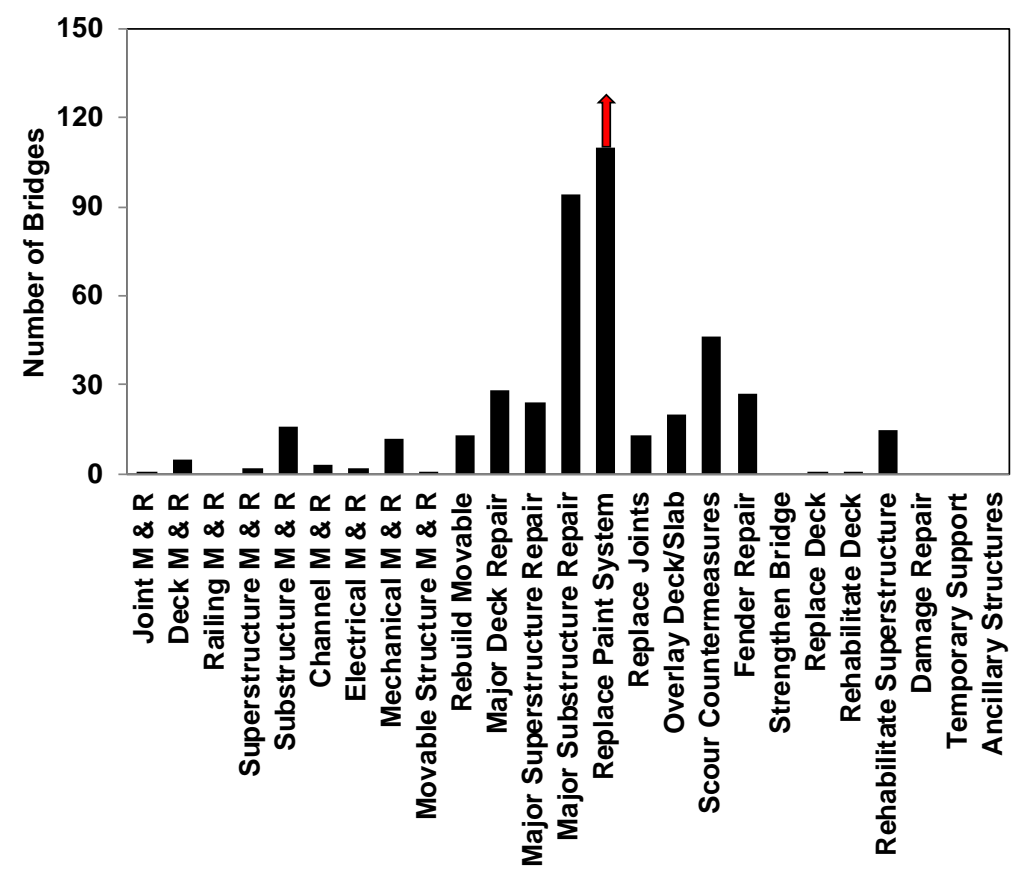

Figure 2.11. Listed Repair Program for the Year 2018 Bridge Work Plan.
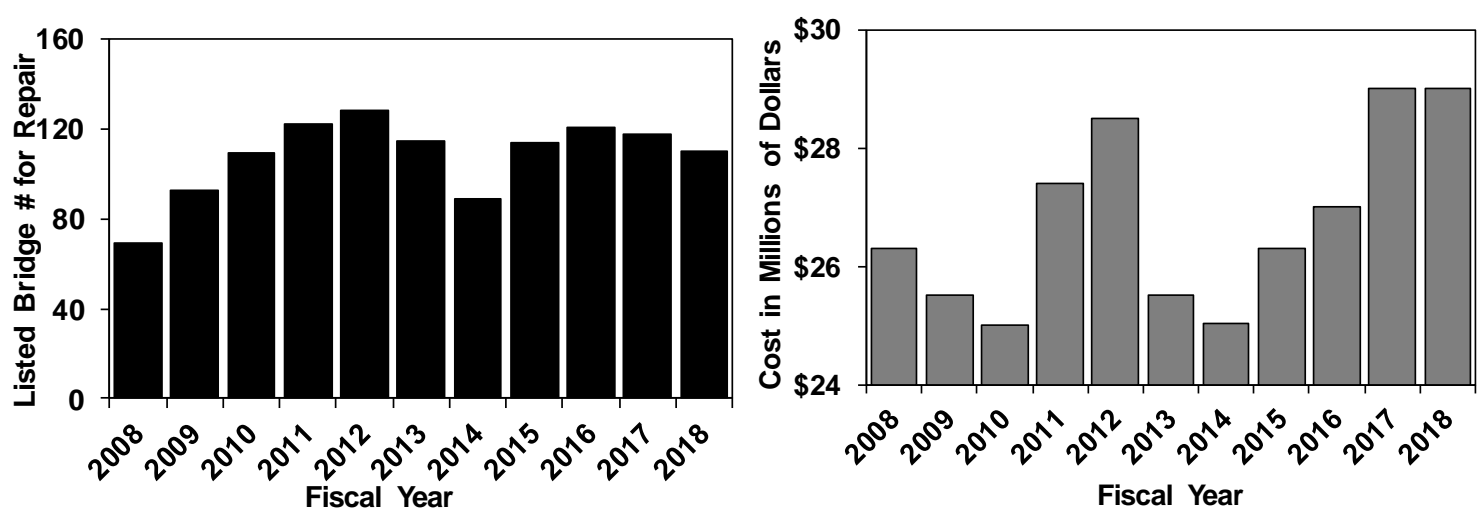

Figure 2.12. FDOT Cost Spent for Steel Bridge Repainting (Work Plan 08-18).

\subsection{Coating Failure}

There are several reasons involved in the failure of complex and sophisticated materials like protective coatings. Some of these reasons include inappropriate coating selection, improper application, failure to cure properly, lack of protection against corrosive agents and mechanical damages. There are many failures of coatings due to the incompatibility of the resins, pigments, and other ingredients in the coating formulation 
to provide desirable characteristics. If the selected coating is formulated inadequately, the coating will most likely fail regardless of all efforts made in an optimal application. The failure type can be divided into three broad categories as formulation-related failures, adhesion-related failures, and substrate -related failures.

It has been estimated from past experience that $70 \%$ of all coating deterioration has resulted from poor or inadequate surface preparation (Dudley, 2003). This can in part lead to adhesion-related failure at the interface between the adhesive and the adherent, or the material to be bonded. Also, a substantial percentage of coating failures are related to the substrate. Coating failure by weathering usually results from condensation and may be increased by absorption of moisture by wind-born salts and also for accumulated debris. Specific types of formulation-related, adhesion-related, and substrate-related failures are presented in Tables 2.2-2.4, grouped by failure appearance, cause, and problem prevention.

Table 2.2. Formulation Related Failures (Bayer \& Zamanzadeh, 2004)

\begin{tabular}{|c|c|c|}
\hline $\begin{array}{c}\text { Organic Coating } \\
\text { Failures }\end{array}$ & Failure Appearance & Cause of Failure \\
\hline Chalking & $\begin{array}{l}\text { Surface soft \& powdery. } \\
\text { Easily wiped away. }\end{array}$ & $\begin{array}{l}\text { UV degradation of resin. } \\
\text { Improper pigmentation. }\end{array}$ \\
\hline Erosion & $\begin{array}{l}\text { Similar to chalking. High spot removal } \\
\text { \& brush marks. }\end{array}$ & $\begin{array}{l}\text { Chalking \& surface } \\
\text { weathering. }\end{array}$ \\
\hline Checking & $\begin{array}{l}\text { Uneven, small, noncontinuous coating } \\
\text { fissures. }\end{array}$ & $\begin{array}{c}\text { Surface stresses caused by } \\
\text { shrinkage. }\end{array}$ \\
\hline Alligatoring & $\begin{array}{l}\text { Large macro-cracking and cross- } \\
\text { hatching. }\end{array}$ & $\begin{array}{c}\text { Internal stresses with greater } \\
\text { surface shrinkage. }\end{array}$ \\
\hline Cracking & $\begin{array}{l}\text { Small breaks in the coating to the } \\
\text { substrate of various geometries. }\end{array}$ & $\begin{array}{l}\text { Stresses due to continued } \\
\text { polymerization/oxidation. }\end{array}$ \\
\hline Mud Cracking & Large macrocracking and curling. & $\begin{array}{l}\text { Rapid drying of highly filled } \\
\text { coatings. }\end{array}$ \\
\hline Wrinkling & $\begin{array}{c}\text { Furrows and ridges in the coating } \\
\text { surface. }\end{array}$ & $\begin{array}{l}\text { Surface dries more quickly } \\
\text { than underlying coating. }\end{array}$ \\
\hline Biological Failure & $\begin{array}{l}\text { Softening or slime reaction. Blotchy } \\
\text { brown or black spots. }\end{array}$ & $\begin{array}{l}\text { Bacterial or fungal } \\
\text { degradation. }\end{array}$ \\
\hline
\end{tabular}




\section{Continuation of Table 2.2. Formulation Related Failures}

\begin{tabular}{ccc}
\hline Organic Coating Failures & Failure Appearance & Cause of Failure \\
\hline $\begin{array}{c}\text { Inorganic Zinc Coating } \\
\text { Failure }\end{array}$ & Fallowing, graying, or darkening. & $\begin{array}{c}\text { Weathering or chemical } \\
\text { reaction. }\end{array}$ \\
\hline Checking & $\begin{array}{c}\text { Fine visible or microscopic checks } \\
\text { that do not penetrate to substrate. }\end{array}$ & $\begin{array}{c}\text { High zinc pigment/binder } \\
\text { ratio. Rapid drying conditions. }\end{array}$ \\
\hline Mud Cracking & Fine to large segments flaking from \\
the surface. & $\begin{array}{c}\text { Too thick application. Too } \\
\text { rapid drying. }\end{array}$ \\
\hline Pinpoint Rusting & $\begin{array}{c}\text { Pinpoint spots of corrosion, } \\
\text { progressing from a few per square foot } \\
\text { to almost continuous. }\end{array}$ & $\begin{array}{c}\text { Improper zinc/binder ratio. } \\
\text { Uneven coating thickness. }\end{array}$ \\
\hline Chemical & $\begin{array}{c}\text { Pinpoint spots of corrosion } \\
\text { progressing limited to continuous } \\
\text { rusting. }\end{array}$ & $\begin{array}{c}\text { Acid or alkali reaction on both } \\
\text { silicate binder and on metallic } \\
\text { zinc. }\end{array}$ \\
\hline Pitting in sea Water & Strong anode forms in breaks in the \\
& coating & $\begin{array}{c}\text { Reaction of chemicals with } \\
\text { inorganic zinc or galvanizing } \\
\text { surface, causing it to become } \\
\text { inert and thus a massive } \\
\text { cathode. }\end{array}$ \\
\hline
\end{tabular}

Table 2.3. Adhesion Related Failures (Bayer \& Zamanzadeh, 2004)

\begin{tabular}{cll}
\hline Coating Failures & \multicolumn{1}{c}{ Failure Appearance } & \multicolumn{1}{c}{ Cause of Failure } \\
\hline Blistering & $\begin{array}{l}\text { Dome-like raised area containing } \\
\text { moisture or other liquids. }\end{array}$ & $\begin{array}{l}\text { Contamination on the surface } \\
\text { prior to painting or coating. }\end{array}$ \\
\hline Peeling & Smooth surface and hanging in shreds. & $\begin{array}{l}\text { Reaction of coating with the } \\
\text { substrate and loss of adhesion }\end{array}$ \\
\hline Flaking or Scaling & $\begin{array}{l}\text { Fine to large segments removed from the } \\
\text { surface }\end{array}$ & $\begin{array}{l}\text { Due to internal shrinkage of } \\
\text { coating and less adhesion. }\end{array}$ \\
\hline Inter-coat Delamination & $\begin{array}{l}\text { Topcoat does not adhere to the } \\
\text { undercoat }\end{array}$ & $\begin{array}{l}\text { Contaminated surface and } \\
\text { over cured undercoat. }\end{array}$ \\
\hline Undercutting & $\begin{array}{l}\text { Corrosion tends to build up } \\
\text { undercoating, at breaks, edges or } \\
\text { holidays. }\end{array}$ & $\begin{array}{l}\text { Poor adhesion due to surface } \\
\text { contamination, smooth surface } \\
\text { or lack of compatibility with } \\
\text { the surface. }\end{array}$ \\
\hline
\end{tabular}

Table 2.4. Substrate Related Failures (Bayer \& Zamanzadeh, 2004)

\begin{tabular}{ccc}
\hline Coating Failures & Failure Appearance & Cause of Failure \\
\hline Previously Used Steel & $\begin{array}{c}\text { Blistering, rust, tubercles, loss of } \\
\text { adhesion. }\end{array}$ & $\begin{array}{c}\text { Retention of minute amounts } \\
\text { of corrosion product; even } \\
\text { after the abrasive blast. }\end{array}$ \\
\hline $\begin{array}{c}\text { Galvanized or Metallic Zinc } \\
\text { Surface }\end{array}$ & $\begin{array}{c}\text { White zinc corrosion product forming } \\
\text { undercoating or breaking through. }\end{array}$ & $\begin{array}{c}\text { Formation of zinc salts } \\
\text { underneath the coating. }\end{array}$ \\
\hline
\end{tabular}




\subsection{Coating Repair}

Preventative maintenance of bridge coating can prolong the service life and prevent more costly damage. The level of maintenance is usually determined by the condition of the coating, but the maintenance strategy is influenced by the ease of access, removal of accumulated debris and washing of contaminants. At first, the condition of the existing coating as coating type, the proportion of coating failure, adherence of the existing coating, coating thickness, coating age as well as the condition of the steel substrate is assessed. Based on the coating condition assessment, a repair decision should be made with consideration of the cost to perform the surface preparation and painting of the bridge. There are several strategies available for the maintenance of steel bridge coatings, such as spot painting, overcoating and full removal or replacement of the existing coating system. Apart from full removal or replacement, the other coating maintenance strategies can extend the service life of existing coating systems and delay major maintenance requirements. For maintenance decisions, the existing coating condition, as well as the condition of the underlying steel substrate, must be carefully assessed to reduce the risk of failure. Repainting a bridge, or even performing paint repairs on a bridge, can involve expensive traffic control and rigging. That is why the right coating system is critical to bridge owners.

\subsubsection{Spot Painting}

Spot painting is applicable when the existing protective coating systems fail over a very limited surface area. In spot painting, only the rusted and delaminated area is cleaned, and new paint is applied over that area to slow the deterioration process and thus extend the life of the paint system and the painted element. To upgrade by spot painting 
the existing coating needs to have less than $10 \%$ surface deterioration or rust grade 4 according to ASTM D160 and a satisfactory adhesion or adhesion classification 2B according to ASTM D3359 (Tam and Stiemer 1996). Spot painting may involve a high unit cost relative to the other painting maintenance strategies but relatively small areas could result in lower total costs.

\subsubsection{Overcoating}

Overcoating involves partial removal of existing paint and application of new coatings over a mixed substrate of existing paint, bare steel, and rusted surfaces. Overcoating is an alternative to full removal and replacement of a failed existing paint system. Overcoating processes vary extensively depending on the condition of the

existing steel and paint system and the specified surface preparation and new coating system. Overcoating is applicable if the existing coating exhibits less than 20 percent deterioration, has a dry film thickness of 5-20 mils and has satisfactory adhesion (Chong and Yao, 2007). Coating selection is even more complicated for overcoating because then not only is it necessary to determine the properties of the coating from the standpoint of the exterior exposure, but also the coating must be satisfactory over the existing coating on the structure. Many failures have occurred due to a lack of attention to the properties of the undercoat and adhesion characteristics of the new repair coating. (Munger and Vincent 2014)

\subsubsection{Repainting}

Repaint is the complete removal of the existing coating system followed by appropriate cleaning of the old coating and application of a new coating system [SSPC]. When the condition of the existing coating system has reached the condition that it is not 
possible to do spot painting or overcoating because of existing coating conditions or considering the comparative repair cost, repainting is the nominal option. Repainting is the most expensive maintenance option. For repainting of an existing bridge appropriate surface preparation and exposure to adverse environmental exposure as well as time is the most critical phenomenon based on the type of repair coating.

\subsection{Application of Bridge Coating}

The performance of the bridge coatings can deteriorate due to application quality and severity of environmental exposure (Myers et al., 2010). Failure of coated surfaces is intrinsic and time to failure is the critical dimension for the bridge owner. The method of application and the conditions under which paints are applied have a significant effect on the quality and durability of new as well as the repair coating performance. Improper surface preparation and the existence of contaminants are detrimental for the durability of bridge coating. But coatings must be field applied in which it is difficult to avoid harmful environmental impurities such as soluble salts, temperature, and humidity.

\subsubsection{Surface Preparation}

Surface preparation is the essential first step before the application of the coating and the most important factor affecting the total success of a corrosion protection system. The performance of a coating is significantly influenced by its ability to adhere properly to the substrate material. The surface preparation process not only cleans the steel but also introduces a suitable profile to receive the protective coating. It has been estimated that 60 to $80 \%$ of all premature coating failures are the result of inadequate or improper surface preparation (Prasanna, 2016). According to FDOT specification all surfaces to be coated should be clean, dry, and free from oil, grease, dirt, dust, soluble salts, corrosion, 
peeling coating, caulking, weld spatter, mill scale and any other surface contaminants by using the SSPC/NACE standard according to the coating specification as shown in Table 2.5.

Table 2.5. List of SSPC and NACE Standards Specifications for Surface Finish.

\begin{tabular}{ccc}
\hline Type & SSPC Standard & NACE Standard \\
\hline Solvent cleaning & SP 1 & \\
\hline Hand tool cleaning & SP 2 & \\
\hline Power tool cleaning & SP 3 & \\
\hline White metal blast cleaning & SP 5 & NACE No. 1 \\
\hline Commercial blast cleaning & SP 6 & NACE No. 3 \\
\hline Brush-off blast cleaning & SP 7 & NACE No. 4 \\
\hline Near-white blast cleaning & SP 10 & NACE No. 2 \\
\hline High and Ultrahigh Pressure Water & SP-12 & NACE No. 5 \\
Jetting & & \\
\hline $\begin{array}{l}\text { SSPC }=\text { Society for Protective Coatings; } \\
\text { NACE }=\text { National Association of Corrosion Engineers; }\end{array}$ &
\end{tabular}

\subsubsection{Effect of Chloride Contamination}

Coatings applied on surfaces contaminated with soluble salts do not provide the anticipated service life; this is the primary reason for premature coating failure at the coating/steel interface. There are numerous sources of soluble salts for steel infrastructure deterioration. Atmospheric pollutants are the most common source and among them, marine chlorides have received the greatest attention because of their detrimental effect on coating failure. The de-icing salts and contaminants from sandblasting are also a potential source of soluble salts (Morcillo 2003). The detrimental effect occurs in practice, predominantly when the metal surface has been exposed for some time prior to painting to an aggressive environment. The detrimental effect of soluble salt concentration on the metal coating interface was first reported by Mayne in 1959. He mentioned that when the coating is applied over a rusty surface and salt concentration exceeds a certain threshold value the system is prone to premature deterioration as well as 
subjected to accelerated corrosion rate (Mayne 1959). Fuente et al. (2006) reported that the presence of hygroscopic salts especially chlorides and sulfates, at the coating/steel interface promotes osmotic blistering of the coating and under film metallic corrosion. Loss of adhesion, cathodic disbondment, scribe creep and a decrease in the adhesion/cohesion strength of the coatings are also reported as a consequence of painting over rusty surface contaminated with soluble salts. Chlorides react chemically with steel and form corrosion nests or cells that concentrate in the pits of steel and can cause accelerated degradation of the coating system (Appleman, 1987). Research by Kentucky Transportation Center also reported that the presence of chloride is the primary cause of coating failure (Meade et. al. 2010). Federal Highway Administration demonstrated that test panels exposed in industrial and marine exposures accumulated substantial surface deposits of chlorides in less than 6 months, which can lead to the deterioration of the paint system in a very short period of time. (Appleman, 1987). So, it was a focus of research in the last couple of decades to fix the limit for critical threshold chloride value. However, it is very difficult to set a unique limit since susceptibility to soluble salt deterioration varies with coating type, thickness as well as exposure environment which control osmotic water migration (Fuente et. al., 2006). Alblas and Londen (1997) concluded that due to lack of consensus on precise contamination levels, it is difficult to set an acceptable level of the upper limit of soluble salt contaminants and also a wide dispersion of the maximum allowable chloride concentrations has been reported from experimental studies. Some research reported the detrimental effects due to chloride contamination as low as $0.5 \mu \mathrm{g} / \mathrm{cm}^{2}$, whereas no harmful consequence was found by some researchers even with a high concentration of $127 \mu \mathrm{g} / \mathrm{cm}^{2}$. Fuente et. al., 2006 
summarized the available experimental findings for the threshold chloride level (Figure 2.13). As can be seen, the reported threshold limit could be as high as $50 \mu \mathrm{g} / \mathrm{cm}^{2}$.

Previous research also reported that different coating system can withstand the different level of residual salt, the performance was preeminent for zinc-rich coating system and tolerance limit also vary with the exposure environment such as atmospheric or immersion (Axelsen and Knudsen, 2011).

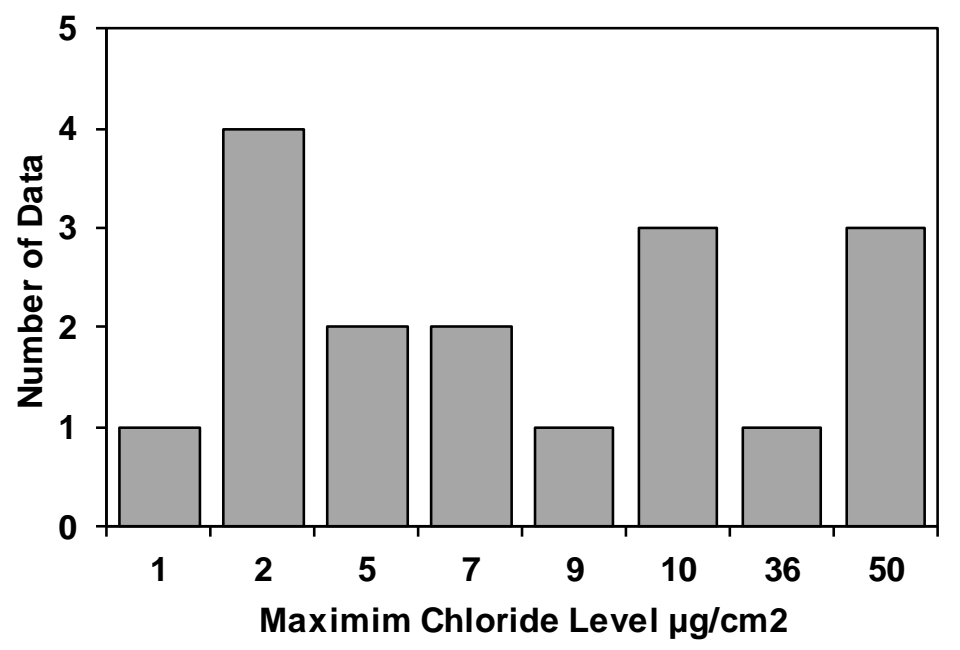

Figure 2.13. Experimental Threshold Chloride Level Data (Fuente et. al., 2006).

\subsubsection{Effect of Relative Humidity and Moisture}

Some level of water is always available in the atmosphere in the form of humidity or moisture. The relative humidity is an important consideration during coating application for the long-term coating durability. The presence of high humidity conditions can interfere with the curing process, increase to the formation of rust blooms on the metal surface and result in coating blistering and delamination. On the other hand, if the coating is applied in too dry conditions, the top surface will cure leaving the lower coating layers uncured. Prasanna (2016) reported that high atmospheric humidity enhances the condensation of moisture on the surface. Painting over the surface 
condensation often causes the formation of rust bloom on the metal surface, resulting in blistering and delamination and consequently total coating failure. Humidity has long been known to play a significant role in the atmospheric corrosion of metals too. The coating degradation mechanism by the influence of moisture can be explained by the following mechanism.

The water $\left(\mathrm{H}_{2} \mathrm{O}\right)$ molecules are very small in size consisting of one oxygen and two hydrogen atoms. Water molecules are also polar in nature as oxygen has a high electronegative attraction to other polar molecules, including itself. Water can readily penetrate into microscopic pores, holidays, cracks, and defects inherent in almost all coating systems. Water molecules fill any free space left by the solvents and other materials that have migrated from the coating during application and curing. Additionally, due to the polarity of the water molecule, water can be drawn into the coating if there are any polar solvents, polar groups, or polar materials retained or comprising the dry film. Thus, the presence of ester groups, ether linkages, carboxyl groups, and other polar groups within a coating resin can draw water into the paint. Once water molecule penetrates the paint film, it separates loose bonds (polar bonds) holding the resin particles together. The molecules become attracted to each other and the volume of the coating expands. Swelling by moisture penetration into a coating film occurs with virtually all coating materials except those that are relatively impervious to water permeation, penetration, and swelling due to their dense molecular crosslinking or the tight polar bonding between molecular chains.

Gradual reduction of barrier properties of the coating system and eventually the protection against corrosion, due to the prolonged contact of the adsorbed water on the 
surface with the corrosive environment was reported by Zhang et. al. (2016). Also, the presence of pollution gases such as $\mathrm{SO}_{2}, \mathrm{H}_{2} \mathrm{~S}, \mathrm{NH}_{3}$, etc. in the atmosphere, particularly at high relative humidity, strongly accelerates the corrosion of metals (Dehri and Erbil, 2000). The humidity range between $75 \%$ and $95 \%$ is the most favorable condition for significant under film corrosion growth (LeBozec et al., 2004). Hygroscopic $\mathrm{NaCl}$ absorbs moisture from the atmosphere when the relative humidity is above $76 \%$ and can stimulate corrosion through osmotic action. (Wise et al., 2007).

\subsection{Exposure Environment in Florida}

Structures located over or within 2500 feet of a body of water containing chloride above $2000 \mathrm{ppm}$ are designated as marine structures and other structures are considered non-marine structures. Further bridge environments are classified as Slightly Aggressive, Moderately Aggressive, or Extremely Aggressive environments according to the chloride content as shown in Figure 2.14 (FDOT, 2017). Marine environments are generally considered the most corrosive environments due to the amount of sea salt carried by winds. The deposition of salts on materials is characterized by proximity to the ocean and salt-laden air. The corrosiveness of a marine environment depends on the topography of the shore, distance from the shore, prevailing winds and relative humidity. 


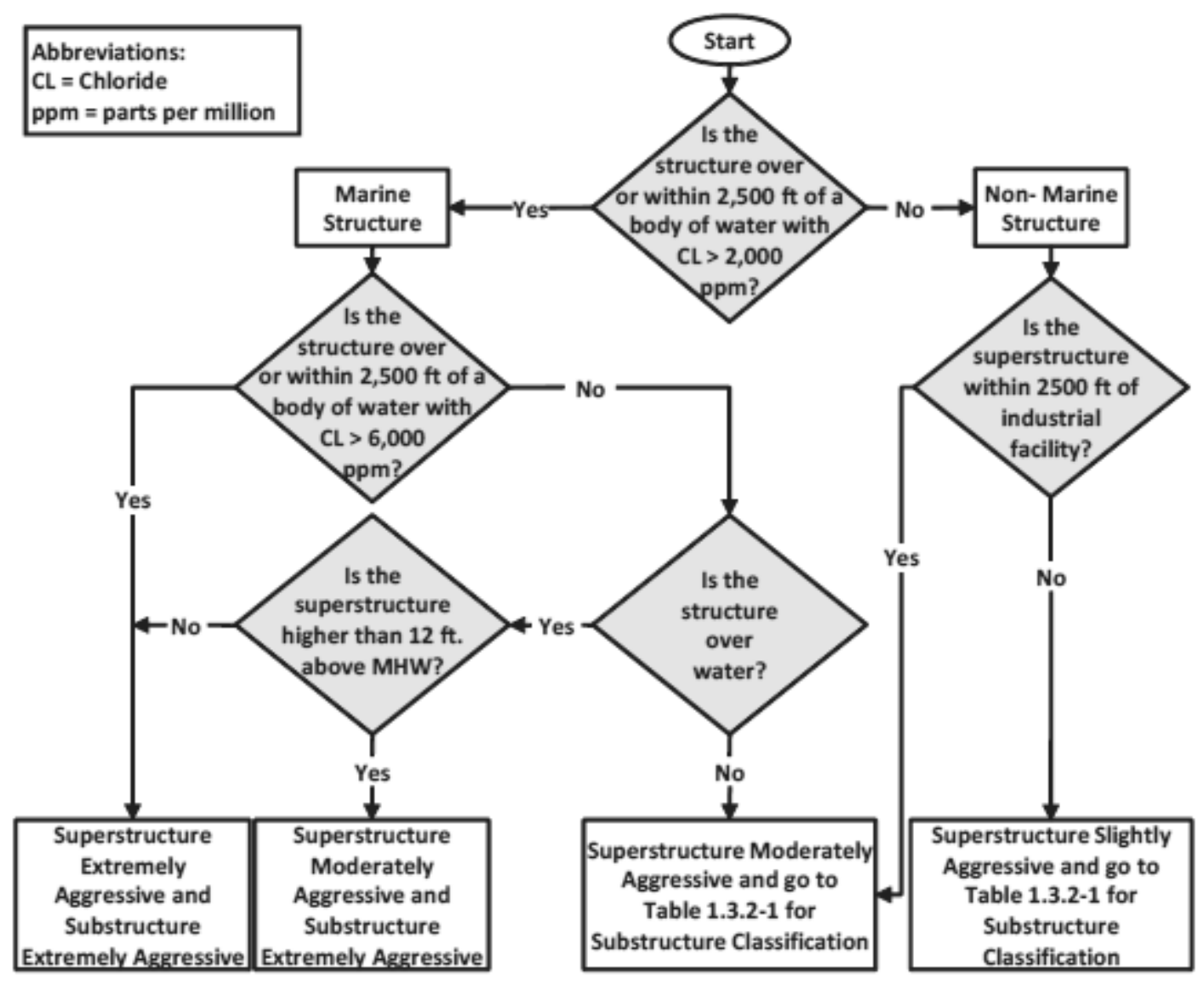

Figure 2.14. Environmental Classification of Structures (FDOT, 2017).

From the chloride ion deposition and atmospheric concentration map (NADP 2015), it is clear that atmospheric chloride level is comparatively higher in the Florida environment and can be detrimental to bridge coating performance as shown in Figure 2.15. Figure 2.16 shows a map of collected chloride data (FDOT, 2017) and the associated aggressive exposure environment based on chloride level. The relative humidity of Florida typically ranges from $41 \%$ to $94 \%$ over the course of the year and maintaining a daily average humidity of $75 \%$ (Zierden, 2014). This humidity level is susceptible to coating degradation and subsequent failure. 

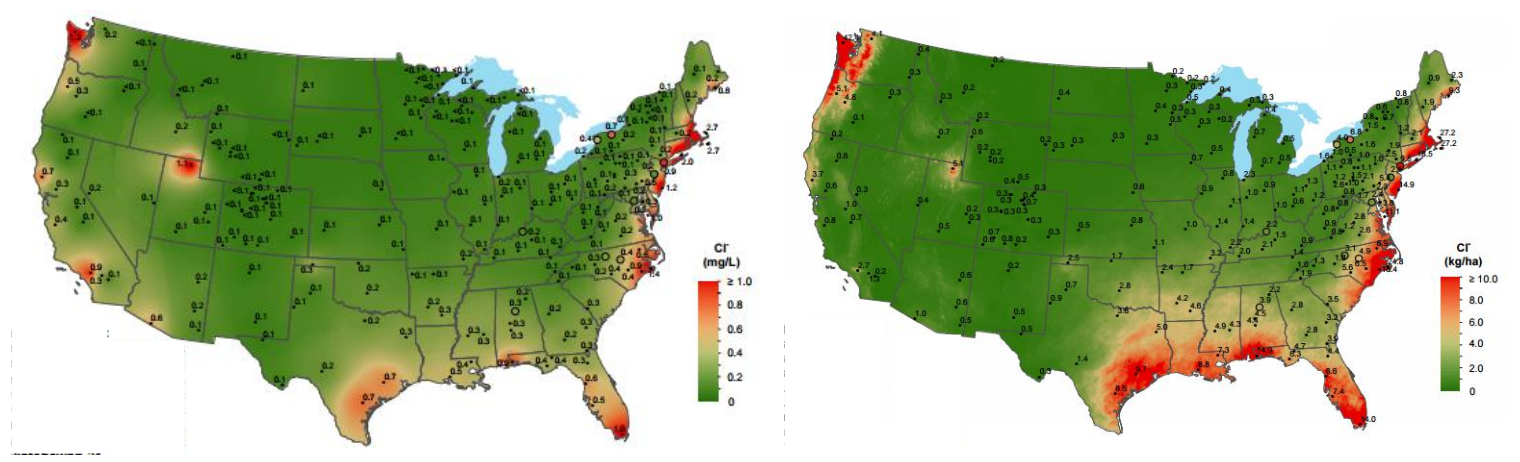

Figure 2.15. Chloride Ion Deposition and Concentration, (NADP, 2015).

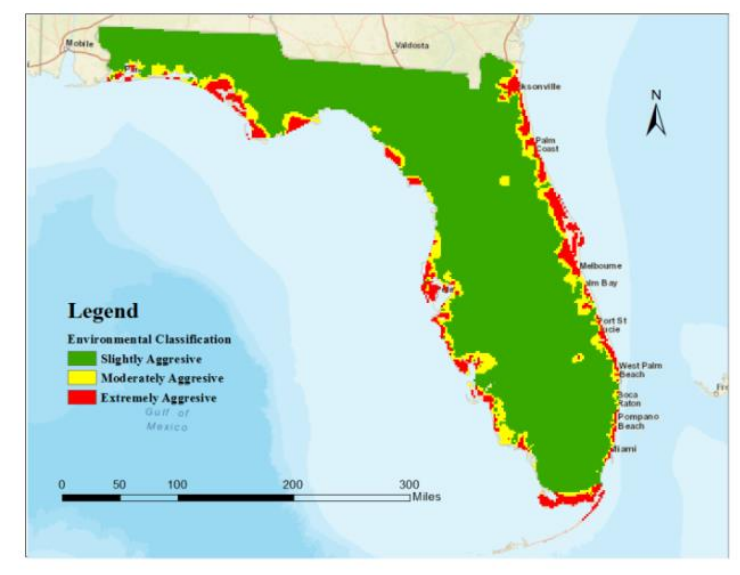

Figure 2.16. Exposure Environment based on Chloride Exposure.

Figure 2.17 shows the evidence of a reduction in corrosion rates from beach sites with an increase in distance (Morrison, 1980). Salt is deposited on steel surfaces by marine fog and windblown spray droplets with a rate of higher than $15 \mathrm{mg} / \mathrm{m}^{2}$ per day. This contamination induces severe corrosion at relative humidity exceeding about $55 \%$. (Syed, 2006). 


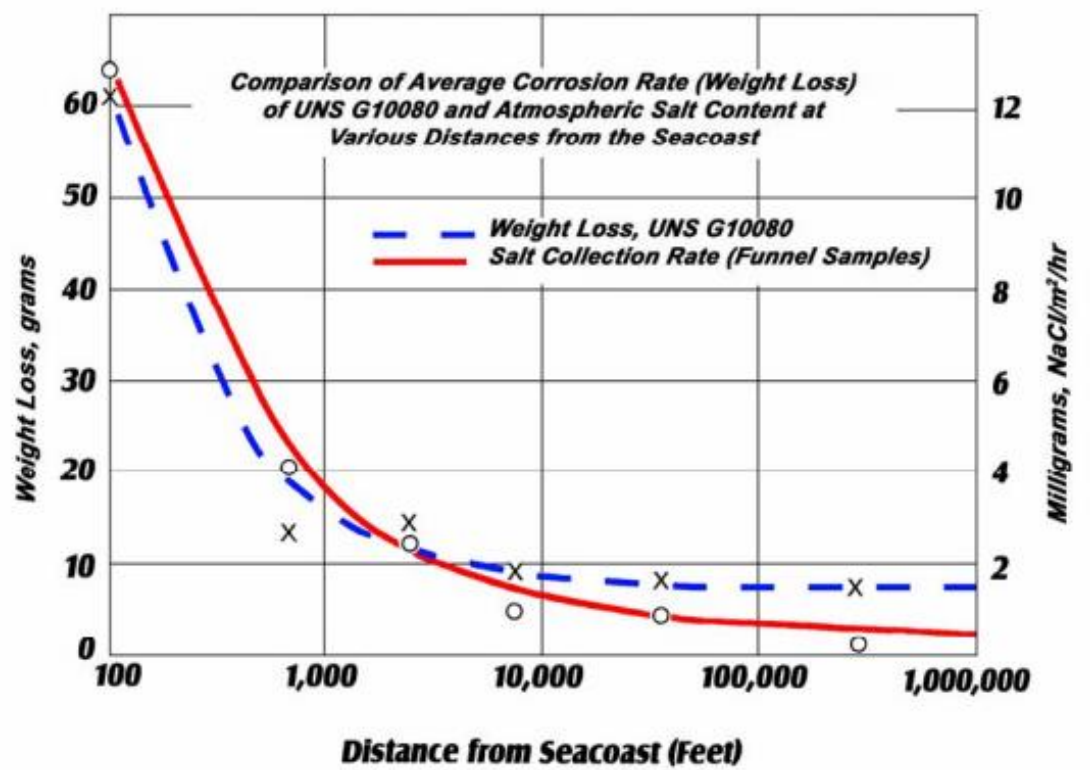

Figure 2.17. Corrosion Rate with Distance from the Ocean (Morrison, 1980).

\subsection{Repair Materials}

The selection of compatible coating material and the corresponding level of surface preparation is the critical parameter for proper protection from its exposure environment. Figure 2.18 shows survey results by transportation research synthesis for the typical maintenance coating system used for maintenance (TRS, 2014). For spot painting, epoxy mastic and polyurethane finish coats are often specified either with or without an epoxy penetrating sealer. With overcoating, some additional barrier protection is provided as well as uniformity of appearance. For repainting, typically an organic zincrich primer and epoxy midcoat with a polyurethane or polysiloxane finish coat, or moisture-cured urethane zinc primer with two more coats are used. 

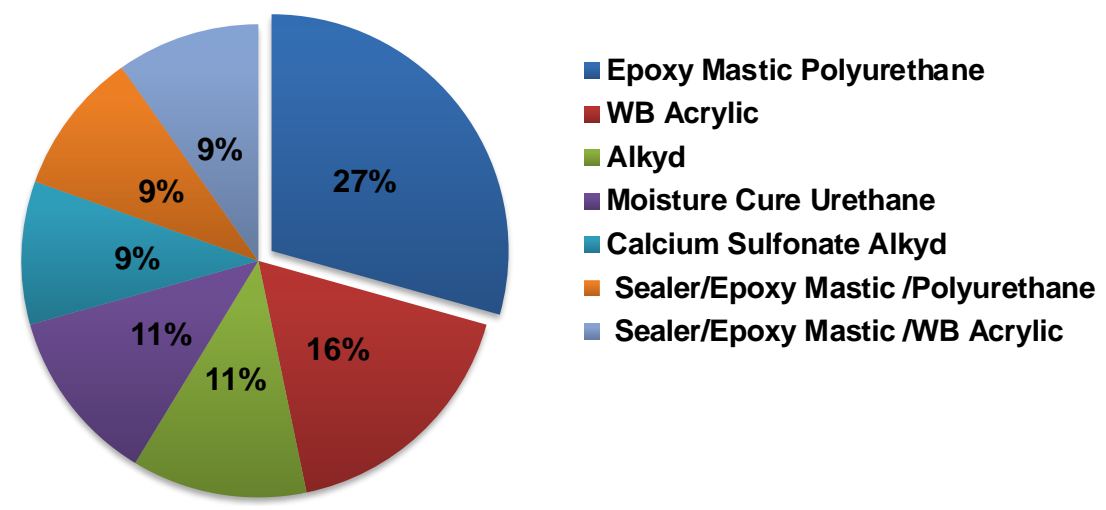

Figure 2.18. Typical Painting Systems Used for Maintenance (TRS, 2014).

\subsection{Improvement of ZRP Coating System}

Coating system components that can improve the effectiveness of ZRP to provide galvanic protection of steel by enhancing the electrical continuity of the zinc primer have been explored. Several studies have addressed various intrinsically conductive pigment including carbon nano-particles to the resin with zinc particles to improve conductivity as Nano-ZnO, (Li and Castaneda (2018), Schaefer and Miszczyk, (2013) and Praveen et al., (2007)).

Among carbon nano-particles, nanotubes have gathered interests for its mechanical, electrical and thermal properties after being introduced by Iijima (Iijima, 1991). Carbon Nanotubes (CNTs) are nanometer-scale tubular structures consisting entirely of carbon atoms as shown in Figure 2.19. CNTs are mainly available in elemental carbon forms, including single-walled nanotubes (SWNTs) and multi-walled nanotubes (MWNTs). MWNTs exhibit extraordinary mechanical as well as electrical properties. A single MWCNT can withstand $~ 63 \mathrm{GPa}$ tensile strength and this outstanding mechanical strength is due to the presence of high bond strength of carbon-carbon sp2 double bond $(\mathrm{C}=\mathrm{C})$ which are stronger than the sp3 bonds found in diamonds. The multi-walled 
carbon nanotubes are stiffest among all of them (Praveen et al. 2007). These carbon tubes are self-assembled into a rope-like structure with high toughness and flexibility which make them promising reinforcing materials to integrate into the epoxy matrix to improve the mechanical performance.
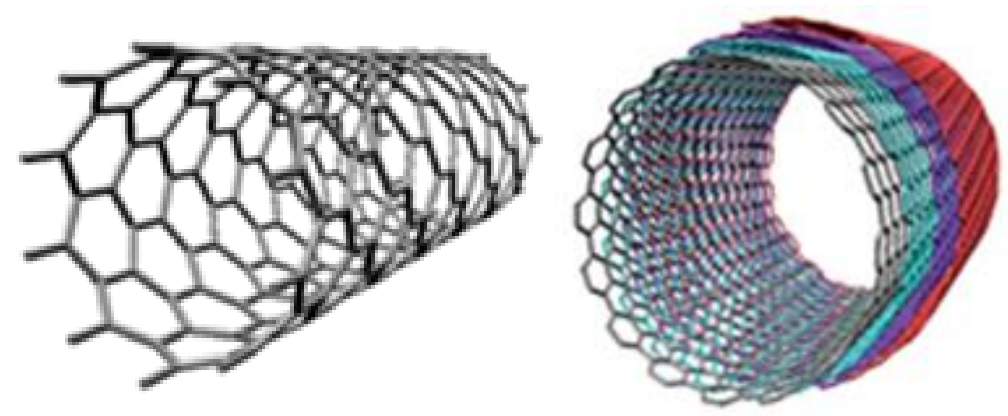

Figure 2.19. Structure of Carbon Nano-particles (Zhang, et. al., 2010).

\subsubsection{Nano-particle Enriched ZRP (NPE-ZRP) Coating}

A new series of corrosion-control coatings containing multi-wall CNTs with sacrificial metal pigments (e.g., Zinc) is commercially available for bridge applications and is the material of interest in the research proposed here. These coatings were advertised to have enhanced strength and electrical conductivity for corrosion inhibition via cathodic protection due to the inclusion of CNTs. This system is referred here as a nano-particle enriched zinc-rich primer (NPE-ZRP) as shown in Figure 2.20.

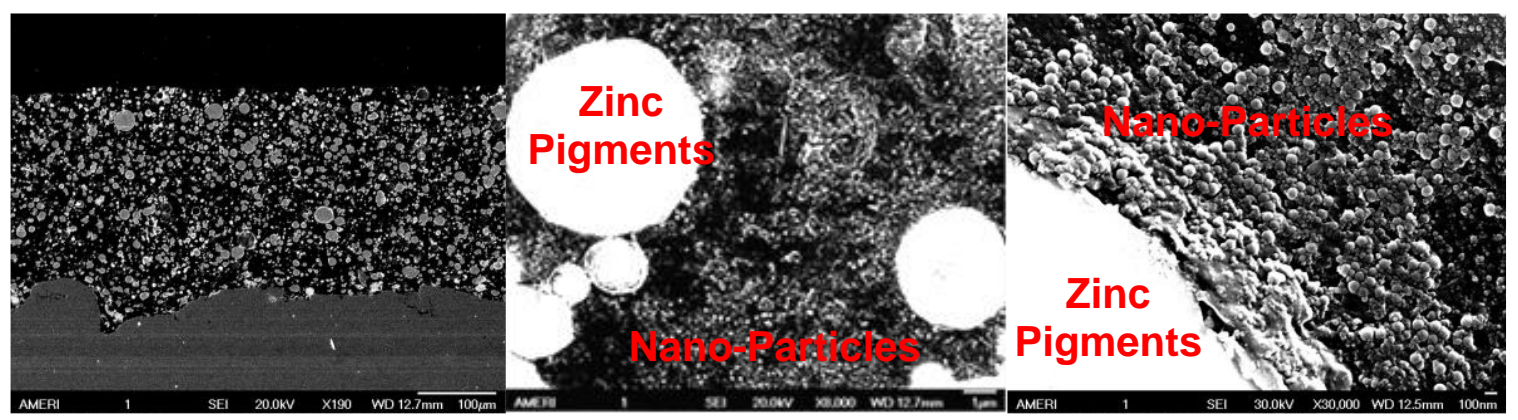

Figure 2.20. Nano-Particle Enriched Zinc-Rich Primer (NPE-ZRP) Coating. 
According to industry literature, NPE-ZRP is a solvent-based, two-component zinc and carbon nanotube (CNT) epoxy-polyamide coating. The CNT coating comprises carbon nanotube-reinforced epoxy primer with dispersed zinc followed by a highperformance topcoat system (epoxy, urethane, or CNT/polyaspartic hybrid). The carbon, in the form of fullerene carbon nanotubes, has a unique structure comprised of a cylindrical nanostructure, with each cylinder capped with hemispherically shaped buckyball structures. The intermediate coat is not required for better adhesion and flexibility, due to the reduced pigment concentration by nanotubes. The function of zinc is to provide cathodic protection and the nanotube provides conductivity to the coating matrix. The expected enhanced conductivity was suggested to allow a reduction in the amount of zinc pigment in the coating. It was suggested that the use of carbon nanotubes provides enhanced coating strength and stiffness (Tesla Nanocoating, 2015). These coatings can be applied using standard painting equipment. Thus, the CNT-based twocoat system can provide considerable project cost/time savings compared to conventional three-coat systems. Another cost mitigation consideration of this NPE-ZRP coating is that the epoxy topcoat can be applied within 30 minutes of the primer application, which may reduce a considerable amount of coating application time (Tesla Nanocoating, 2015).

\subsubsection{Performance of NPE-ZRP Coating}

The following section presents a summary of some recent research on ZRP coating by incorporating carbon nano-particles.

An investigation by the US Army showed superior or comparable results with 3coat systems after 7 years of immersion in freshwater. It was stated that the new hybrid 
coating technology combines superior physical performance properties as a barrier coating with a high degree of cathodic protection $(\mathrm{CP})$ as a sacrificial coating and by imparting the nanotubes' stiffness and strength, which provide up to a $400 \%$ improvement in tensile and fatigue strength, and increase impact and abrasion resistance (Drozdz, 2012).

Park and Shon (2015) reported a reduction in corrosion occurrence, from the epoxy zinc-coated carbon steel with increased zinc or MWCNT content, due to its increased conductivity. The addition of MWCNTs increased the adhesion strength of epoxy zinc coatings, with this effect becoming more apparent after hygrothermal cyclic testing.

In recent research, Cubides and Castaneda (2016) investigated the electrochemical characterization of carbon nanotube and zinc-rich epoxy primers on carbon steel in simulated concrete pore (SCP) solutions. They reported beneficial cathodic protection of carbon steel in simulated concrete pore solutions with zinc-rich primers augmented with nano-particles, compared to the traditional zinc concentration. Cathodic protection was due predominantly to the formation of a barrier layer of zinc corrosion products (zinc oxide, zinc hydroxide, and hydrozincite) that increased the total resistance of the system.

Cubides et al. (2016), also studied the influence of zinc content and chloride concentration on the corrosion protection performance of zinc-rich epoxy coatings containing carbon nanotubes on carbon steel in simulated concrete pore environments. They concluded that more than $70 \mathrm{wt} \%$ zinc pigments with the presence of CNTs are required for an efficient galvanic protection process. CNTs also increased the barrier properties of the coating by blocking the microspores and defects in the material. They 
also found that the simulated concrete pore solution with high chloride concentration caused the breakdown of the passive layer, blister formation, and dissolution of zinc corrosion products.

Praveen et al. (2007), studied the corrosion protection of a carbon nanotube and zinc composite coating, obtained by electrodeposition from a sulfate bath containing dispersed carbon nanotubes. An increase in corrosion resistance was observed when the carbon nanotubes were added to the zinc coating, with carbon nanotubes providing a physical barrier and filling micro-holes on the metal surface, which acts as active sites for metal dissolution.

Gergelya et. al. (2007), studied zinc-rich hybrid paint coatings developed with nano-size particles composed of alumina hydrate modified with polystyrene-sulfonate (PSS) doped polypyrrole (PPy) and either purified or functionalized multi-walled carbon nanotubes (MWCNTs). They concluded that CNTs can interconnect the zinc particles and provide higher electrical conductivity with which the percolation threshold can be reached with a lower zinc concentration compared with the traditional zinc-rich epoxy primer.

\subsection{Electro Chemical Basic Principles}

\subsubsection{Important Types of Corrosion}

Some important mechanism of localized corrosion is described in the following paragraphs.

Galvanic corrosion is induced when two dissimilar materials are coupled together in a corrosive environment (Jones, 1996). One of them acts as an anode and another one acts as a cathode. The alloy with more positive or noble potential will be protected by the 
other. So, for galvanic corrosion three conditions need to be satisfied - 1) two electrochemically dissimilar materials, 2) electric connection and 3) electrolyte.

Pitting corrosion is a localized form of corrosion which initiated by localized chemical or mechanical damage to the protective oxide film, low dissolved oxygen concentrations and high concentrations of chloride. Pitting corrosion is an autocatalytic process. Rapid dissolution of metal ions occurs if the reduction of oxygen ion is supported by the surrounding surface. When the concentration of positive metal ion increases the negatively charged chloride ion is attracted by the pit and forms the metal chloride. This metal chloride forms hydrogen ion through the hydrolysis process. Both the hydrogen and chloride ion accelerates the dissolution of metal ions with time. Pitting is considered to be more dangerous than uniform corrosion damage because of its difficulty to detect, predict and design. A small, narrow pit with minimal overall metal loss can lead to the failure of an entire engineering system

Crevice corrosion is a type of localized corrosion with the presence of a stagnant solution in the crevice. Crevice environment may be formed in the small sheltered volume of two similar or dissimilar materials, deposition of mud, sand or other insoluble solids or a non-metallic gasket or packing. Differential aeration and chloride concentration are the two important mechanisms for crevice corrosion. The mechanism of crevice corrosion (Rashidi et al., 2007) is described next.

Corrosion occurs both inside and outside the crevice. The associated anodic and cathodic reactions are shown in Eq. 2.1 and Eq. 2.2 respectively.

$$
\begin{gathered}
\mathrm{M} \rightarrow \mathrm{Mn}^{+}+\mathrm{ne}^{-} \\
\mathrm{O}_{2}+2 \mathrm{H}_{2} \mathrm{O}+4 \mathrm{e}^{-} \rightarrow 4 \mathrm{OH}^{-}
\end{gathered}
$$


The positively charged metallic ions are electrostatically counterbalanced by $\mathrm{OH}^{-}$. The cathodic reaction inside the crevice consumed most of the available oxygen. At the same time, $\mathrm{Cl}^{-}$diffuse into the crevice to maintain minimum potential energy and metal chloride is formed. Finally, the hydrolysis of metal chloride lowers the $\mathrm{pH}$. High chloride ion concentrations lead to lower $\mathrm{pH}$ inside crevice and accelerate the metal dissolution. As a result, more metal ions will be produced that will lower $\mathrm{pH}$ again.

Crevice corrosion of the coated steel sample forms due to the presence of coating defects and the deposits of dirt. The opening of the defect should be sufficiently large for moisture to enter the solution and narrow enough to hold the stagnant solution.

\subsubsection{Corrosion of Steel}

Corrosion of steel involves electrochemical reactions including the oxidation of iron (Eq.2.3) and typically the reduction of atmospheric oxygen (Eq.2.4). The total reaction can be written in the form of (Eq.2.5)

$$
\begin{array}{ll}
\text { at anode } & \mathrm{Fe} \rightarrow \mathrm{Fe}^{2+}+2 \mathrm{e}^{-} \\
\text {at cathode } & \mathrm{O}_{2}+2 \mathrm{H}_{2} \mathrm{O}+4 \mathrm{e}^{-} \rightarrow 4 \mathrm{OH}^{-} \\
& 2 \mathrm{Fe}+\mathrm{O}_{2}+2 \mathrm{H}_{2} \mathrm{O} \rightarrow 2 \mathrm{Fe}(\mathrm{OH})_{2}
\end{array}
$$

As oxygen is readily dissolved in water, the excess oxygen reacts with hydroxide (Eq.2.6) to form the rust

$$
4 \mathrm{Fe}(\mathrm{OH})_{2}+\mathrm{O} 2=2 \mathrm{H}_{2} \mathrm{O}+2 \mathrm{Fe}_{2} \mathrm{O}_{3} \cdot \mathrm{H}_{2} \mathrm{O} \text { (rust) }
$$

\subsubsection{Corrosion of Zinc}

\subsubsection{Uniform Dissolution}

Zinc forms protective layers reacting with carbonates, oxides, or hydrated sulfate depending on the surrounding environment. In dry air, a film of zinc oxide or zinc 
hydroxide is initially formed reacting with atmospheric oxygen. In the presence of moisture (i.e., rain, mist, or dew) and atmospheric carbon dioxide, zinc carbonate forms, which act as a protective layer and inhibit further corrosion. However, the formation and maintenance of these protective layers are governed by the $\mathrm{pH}$ of the associated surrounding environment.

\subsubsection{Wet Storage Stain ("White Rust")}

White rust forms when zinc is exposed to a continuous wet environment with a limited supply of oxygen, carbon dioxide, and chlorides and sulfates. White rust is a crumbly and porous layer of $2 \mathrm{ZnCO}_{3} .3 \mathrm{Zn}(\mathrm{OH})_{2}$ together with $\mathrm{ZnO}$ and voluminous $\beta$ $\mathrm{Zn}(\mathrm{OH})_{2}$.

\subsubsection{Galvanic Corrosion}

When two different metals are in electrical contact and bridged by an electrolyte, current flows from anode to cathode. As a result, the nobler metal tends to be protected by sacrificing the baser metal, which is the principal of galvanic protection of zinc coating. Galvanic protection of zinc coating is the ability to protect exposed steel substrate by the sacrificial corrosion of the zinc pigments. This occurs because zinc is more electronegative than steel in the galvanic series as shown in Table 2.6. 
Table 2.6. Electrode Potential of Metals in Seawater (Jones, 1996).

\begin{tabular}{|c|c|}
\hline Material & Potential (V) \\
\hline Magnesium & -1.48 \\
\hline Zinc & -1.03 \\
\hline Aluminum & -0.79 \\
\hline Cadmium & -0.7 \\
\hline Cast Iron & -0.61 \\
\hline Carbon Steel & -0.61 \\
\hline Stainless Steel: $18 \% \mathrm{Cr}, 8 \% \mathrm{Ni}$ & -0.54 \\
\hline Lead & -0.5 \\
\hline Solder $(\mathrm{Pb} / \mathrm{Sn}, 50: 50)$ & -0.45 \\
\hline Tin & -0.42 \\
\hline Copper & -0.36 \\
\hline Aluminum Bronze & -0.34 \\
\hline
\end{tabular}

In practice, this means that a zinc coating will not be undercut by steel corrosion because the steel cannot corrode adjacent to the zinc coating. The distance over which the galvanic protection of zinc is effectively known as throwing power depends on the ratio of the areas of metal in contact, the duration of wetness and the conductivity of wetness. When completely and continuously wetted, especially by a strong electrolyte relatively large areas of exposed steel will be protected as long as any zinc remains. In the air, where the electrolyte is only superficial or discontinuously present, smaller areas of exposed steel are protected. The electrochemical reactions of zinc galvanic protection are presented below:

Zinc Anode Reaction and Oxidation

$$
2 \mathrm{Zn} \rightarrow 2 \mathrm{Zn}^{2+}+4 \mathrm{e}^{-}
$$

Oxygen Reduction

$$
\begin{gathered}
\mathrm{O}_{2}+4 \mathrm{e}^{-}+2 \mathrm{H}_{2} \mathrm{O} \rightarrow 4 \mathrm{OH}^{-} \\
4 \mathrm{OH}^{-}+2 \mathrm{Zn} \rightarrow 2 \mathrm{Zn}(\mathrm{OH})_{2}
\end{gathered}
$$


Overall Reaction

$$
\mathrm{O}_{2}+2 \mathrm{H}_{2} \mathrm{O}+2 \mathrm{Zn} \rightarrow 2 \mathrm{Zn}(\mathrm{OH})_{2}
$$

\subsection{Test Procedure}

\subsubsection{Three-electrode System}

Three-electrode systems is an electrochemical test arrangement consists of working, counter and reference electrode. The electric current passes through the working and counter electrode to complete the electrochemical cell and the purpose of the reference electrode is to monitor the potential of the working electrode. Electrochemically stable materials are used as a counter electrode to prevent the formation of any product and for the measurement of the potential difference between working and counter electrode.

\subsubsection{Open Circuit Potential (OCP)}

When a metal is immersed in a solution, the electrochemical phenomena of metal ions tend to cross the metal/solution interface depending upon the chemical energy of the ionic system. Conventionally only the positively charged cations can pass through the interface. The negatively charged electrons cannot pass into the solution, and the anions cannot pass into the metal. Consequently, charge accumulation occurs at the interface forming an electrical double layer. At the electric double layer, the metal surface becomes negatively charged because of the accumulation of the electrons and the solution layer near the metal surface becomes positively charged because of the accumulation of cations. The potential difference between the metal and the solution phases under these conditions is called open circuit potential or equilibrium potential difference. In short, the potential (OCP) of metal in solution is the energy released at the time of corrosion. This 
potential difference cannot be measured directly because an electrical connection cannot be made to the solution phase without setting up another electrode potential. The electrode potentials are always measured against a reference electrode whose potential is known on an arbitrary scale such as hydrogen electrode. In other words, open circuit potential may be described as an electric potential at zero current flow (McCafferty, 2010).

\subsubsection{Linear Polarization Resistance (LPR)}

Corroding systems can be characterized by artificially changing its potential from its OCP by a small amount and measuring the corresponding current. From that Rp, polarization resistance can be calculated as the ratio of change in the potential to the amount of required current. Then the corrosion current, $I_{c o r r}$, is calculated by Faradic Conversion (Fontana and Greene, 1986) using Eq. 2.7,

$$
I_{\text {corr }}=\frac{B}{R p}
$$

where the Stern-Geary Coefficient, B, was assumed to be $26 \mathrm{mV}$ for active corrosion conditions. To determine the corrosion rate for the coated steel area from the linear polarization technique is complicated due to the actual affected steel area and other current confinement issues (Broomfield, 1997).

\subsubsection{Electrochemical Impedance Spectroscopy}

Electrochemical impedance spectroscopy testing is a non-destructive method to assess the electrochemical properties of the corrosion system and a method to assess physical coating conditions by a range of sinusoidal signal frequency perturbation to their corresponding electrical analog (Barsoukov \& Macdonald, 2005). 
Like other electrochemical systems, the EIS measurement can be made with the three-electrode system. The working electrode is the metal sample of interest and the potentiostat maintains the desired level of potential with respect to the reference electrode. The uniform distribution of electric current for excitation is maintained by the counter electrode, which eventually completes the full electric circuit. The acquired impedance spectra for a range of frequency is interpreted as that corresponding to an electrical analog to possible physio-electrochemical properties and processes in the corrosion system (Barsoukov \& Macdonald, 2005).

While an intact coating comes in contact with the electrolyte, the electrolyte enters the pore of that coating. The pore resistance, $R_{p o}$ decreases with the passage of electrolyte intrusion. Initially, the pore resistance is considered as infinite. The entire system is represented in the equivalent circuit as shown in Figure 2.21 where $R_{s}$ is the solution resistance and $\mathrm{C}_{\mathrm{c}}$ is coating capacitance.

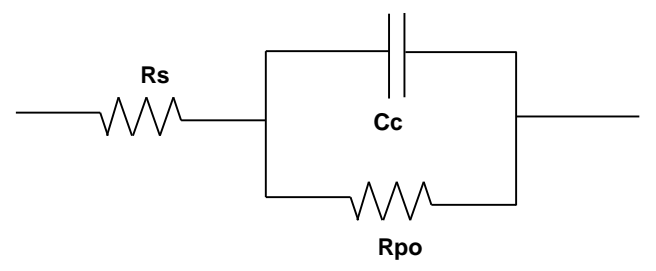

\section{Figure 2.21. Equivalent Circuit of an Intact Coating in Contact with Electrolyte.}

As shown in Figure 2.22, a new phase is included with this equivalent circuit at corrosion reaction which takes place at the metal electrolyte interface. This circuit adds the double layer capacitance, $\mathrm{C}_{\mathrm{dl}}$ proportional to the active metallic area in contact with the electrolyte and resistance element, $R_{P}$ is the polarization resistance. 


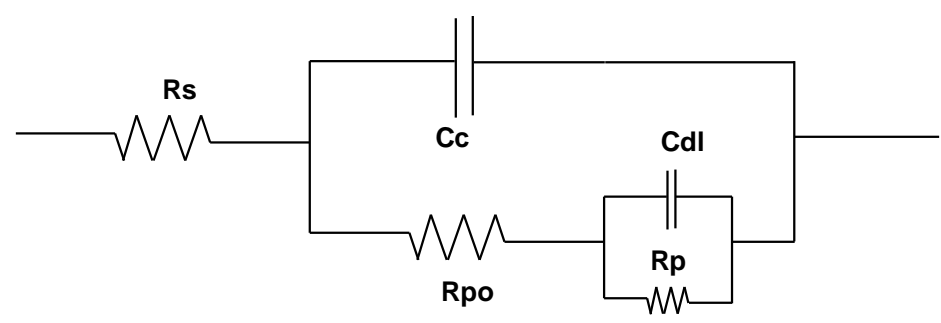

\section{Figure 2.22. Equivalent Circuit of Coating in Contact with Electrolyte by Considering the Base Property of the Steel.}

The coating capacitance Cc is defined by Eq. 2.8,

$$
\mathrm{C}_{\mathrm{c}}=\frac{\varepsilon_{\mathrm{o}} \varepsilon_{\mathrm{r}} \mathrm{A}}{\mathrm{d}}
$$

where $\varepsilon_{\mathrm{o}}$ is the vacuum permittivity or the permittivity of the free space, $\varepsilon_{\mathrm{r}}$ is the relative permittivity or coating dielectric constant, A the coating surface area and d its thickness. Rpo, the pore resistance defined by Eq. 2.9,

$$
\mathrm{R}_{\mathrm{po}}=\frac{\rho \mathrm{d}}{\mathrm{n} \pi \mathrm{r}^{2}}
$$

where the coating defects can be idealized as a distribution of cylindrical pores (Grundmeier et al., 2000) of radius $r, \rho$ is the electrolyte resistivity within the pore, $d$ is the thickness, $\mathrm{r}$ is the pore radius, and $\mathrm{n}$ is the number of pores.

The decrease of $\mathrm{R}_{\mathrm{po}}$, pore resistance can be described as the penetration of electrolyte into the pores and the increase of $\mathrm{R}_{\mathrm{po}}$ may be characterized as delamination of the pore area or formation of new pores.

Non-ideal capacitive behavior and other factors due to heterogeneities including non-uniform current distribution in the coating and metal-electrolyte interface were in part represented by constant phase elements. The total impedance on the first approach was expressed as shown in Equation 2.10. 


$$
Z=R s+\frac{1}{Y_{o c}(j \omega)^{n_{c}}+\frac{1}{R p o+\frac{1}{Y_{o m}(j \omega)^{n_{m}}+\frac{1}{R p}}}}
$$

The solution resistance, Rs, is the resistance between the working and reference electrodes, the pore resistance, Rpo, is the resistance associated with pores and defects in the coating, the polarization resistance, $\mathrm{Rp}$, is a function of the corrosion rate. The impedance of the electrical double layer and the coating capacitance are expressed in the form of constant phase elements $Z_{C P E}=1 /\left(Y_{o}(j \omega)^{n}\right.$ where $Y_{o}$ is the pre-exponential term, $\omega$ is the angular frequency, and $\mathrm{n}$ is a real number $0<\mathrm{n}<1$. The subscripts $\mathrm{c}$ and $\mathrm{m}$ refer to the impedance of the coating and double layer, respectively.

The conventional interpretation of the impedance response of a coated metal interface was assumed as the first approach represented by Nyquist diagram of two semicircles as shown in Figure 2.23. The two semicircles represent two times constant. The first semicircle with radius Rpo/2 represents the coating characteristics and the second semicircle with radius $\mathrm{Rp} / 2$ represents the steel/ coating interface property.

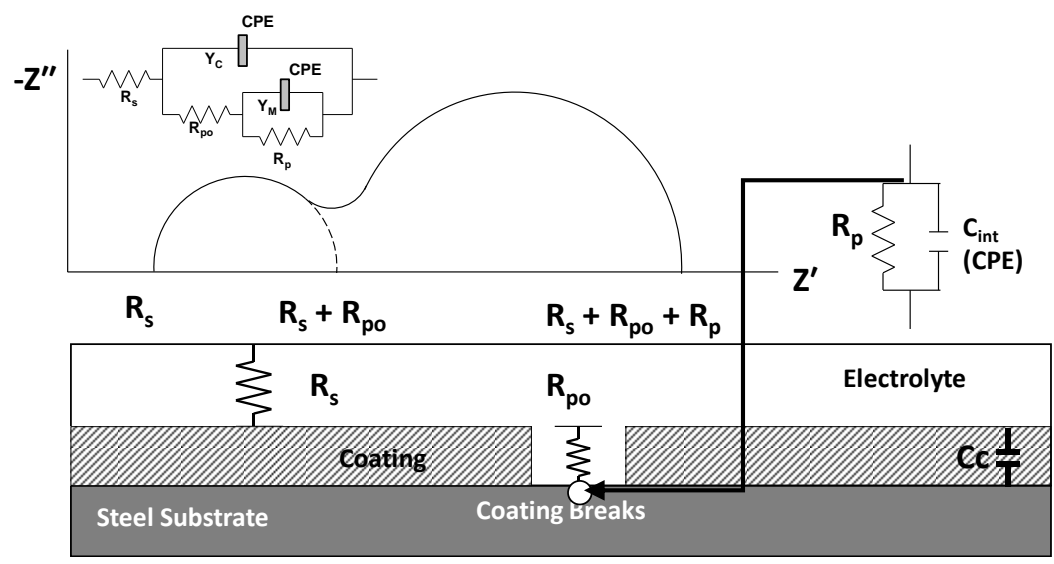

Figure 2.23. Idealized Impedance Diagram and Equivalent Circuit of Coated Metal System with Coating Defect (Sabbir, 2017). 
The CPE element is useful for the circuit fitting of a coating system with non-ideal capacitive behavior. The resolved circuit element cannot characterize the coating capacitance. Only in ideal condition when the $n=1$, coating capacitance can be directly calculated from CPE element. Most of the electrochemical system shows deviation of $\mathrm{n}$ value from unity. The conversion equation of Hsu and Mansfeld can be used to convert the Yo to effective coating capacitance $\mathrm{C}$ as:

$$
\mathrm{C}=\mathrm{Y}_{\mathrm{o}}\left(\omega^{\prime \prime}\right)^{\mathrm{n}-1}
$$

Where $\mathrm{C}$ is the capacitance, Yo is the pre-exponential term, and $\omega^{\prime \prime}$ is the radial frequency at maximum imaginary impedance $n$ real number $(0<\mathrm{n}<1)$. The capacitance of the coating is a water sensitive measurement and expected to change during exposure due to moisture penetration through the coating. Thus, the degradation of the coating can be inferred by the change of coating capacitance.

$$
\text { The capacitance is expressed by } C_{c}=\frac{\varepsilon_{O} \varepsilon_{r} A}{d}
$$

where: Cc coating capacitance, $\varepsilon_{O}$ the permittivity of vacuum or the permittivity of free space (8.854 X 10-12 F.m $\left.{ }^{-1}\right), \varepsilon_{r}$ relative permittivity or coating dielectric constant, A coating surface area and d coating thickness.

The coating capacitance increases due to the water absorption and the volume fraction of water absorbed can be estimated by (Brasher and Kingsbury, 1954) Equation

$$
X_{v}=\frac{\log \left(\frac{C}{C_{O}}\right)}{\log \left(\varepsilon_{H_{2} o}\right)}
$$


where: $X_{v}$ the volume fraction of absorbed water, Co capacitance before water

absorption, $\mathrm{C}$ capacitance at a specific time, $\varepsilon_{\mathrm{H}_{2} \mathrm{O}}$ the dielectric constant of water.

\subsubsection{Surface Wetting Property}

A principal requirement for strong adhesive bonds is that the surface should be clean which confirms a high energy state. Adhesion requires intimate contact of the materials to be joined and increasing the surface roughness of the metal substrate is often used in practice for the bond improvement. These surface properties can change the wetting behavior of the metal substrate with the polymer coating resin. Good wettability is necessary to obtain a durable adhesive connection which determines the ability of a liquid to coat a solid substrate, depending on the interactions between them. The wetting phenomena can be characterized through several thermodynamic parameters such as contact angle, surface energy and work of adhesion.

The contact angle of a liquid is the result of the mechanical equilibrium of a drop resting on a plane solid surface under the action of three surface tensions as shown in Figure 2.14, represented by Young's equation

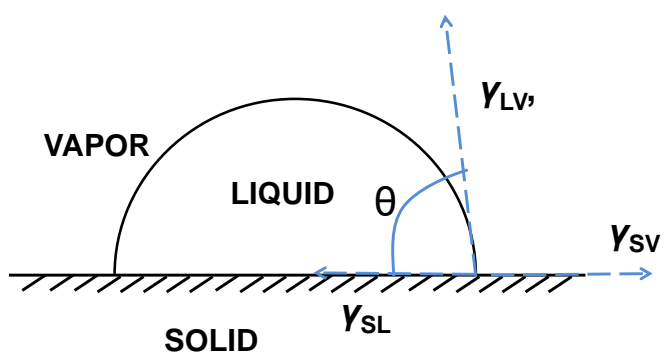

Figure 2.24. Energy Balance of a Liquid Drop Resting on a Solid Surface.

$$
\gamma^{S L}=\gamma^{S V}+\gamma^{L V} \operatorname{Cos} \theta
$$

where $\theta$ is the contact angle and $\gamma^{S L}, \gamma^{L V}, \gamma^{S L}$ is the surface tension coefficients $\left[\mathrm{mJ} / \mathrm{m}^{\wedge 2}\right]$ 
for solid-vapor, vapor-liquid, solid-liquid respectively.

The wettability of a solid surface with a liquid can be defined by reversible work of adhesion $W_{A}$

$$
W_{A}=\gamma^{S V}+\gamma^{L V}-\gamma^{S L}
$$

Substituting the equation of Young to adhesion work dependence the mechanical work of adhesion is defined by the Young-Dupré's equation.

$$
W_{A}=\gamma^{L V}(1+\operatorname{Cos} \theta)
$$

The surface energy of a solid surface can be calculated by measuring contact angles using various methods, including the method of using three well-defined probe liquids following Lifshitz van der Waals-acid based formalism.

$$
\gamma^{L V}(1+\operatorname{Cos} \theta)=2\left[\sqrt{\gamma_{S}^{L W} \gamma_{L}^{L W}}+\sqrt{\gamma_{S}^{+} \gamma_{L}^{-}}+\sqrt{\gamma_{L}^{+} \gamma_{S}^{-}}\right.
$$

The surface energy $\gamma$ is split into $\gamma^{L W}$ and $\gamma^{A B}$,

$$
\gamma=\gamma^{L W}+\gamma^{A B} \text { with } \gamma^{A B}=2\left[\sqrt{\gamma^{+}} \gamma^{-}\right.
$$

Where, the LW stands for Lifshitz-van der Waals interactions that include London dispersion, Keesom dipole-dipole, and Debye induction; the superscript AB represents Lewis acid-base interactions; $\gamma \mathrm{p}$ and $\gamma$ are the Lewis acid (electron-acceptor) and Lewis base (electron-donor) parameters of surface energy, respectively. 


\section{CHAPTER 3}

\section{METHODOLOGY}

\subsection{Performance of the NPE-ZRP Coating System}

NPE-ZRP coating was tested for the application of new steel structures to identify if NPE-ZRP could provide enhanced coupling of the zinc pigments from the primer to the steel substrate from testing in atmospheric exposures with various moisture and salt environments including extended outdoor atmospheric exposures and from salt-fog exposures. Since moisture and salt were expected to be a major factor in the exposure, a set of testing was made by immersing coated steel samples in $3.5 \mathrm{wt} \% \mathrm{NaCl}$ solution. Testing here considered localized coating damage that exposed the steel substrate. Furthermore, it was of interest to identify if nano-particles can extend the connectivity of embedded zinc pigments and to identify polarization behavior. Test conditions to promote the electrochemical activity of the zinc (including both oxidation and reduction reactions) included various levels of electrochemical polarization. In order to assess the effect of nano-particles on zinc electrochemical coupling, steel corrosion development, the extent of zinc consumption, and coating degradation were addressed. A traditional zinc-rich primer (ZRP) was studied to reference the performance of nano-particles.

\subsubsection{Sample Preparation}

Low carbon steel (A36) panels $(76.2 \mathrm{~mm} \times 127 \mathrm{~mm} \times 3.2 \mathrm{~mm})$ from the same material provider were used for testing. The chemical composition of the steel plate is presented in Table 3.1. NPE-ZRP and ZRP coating materials were applied on sandblasted steel coupons following SSPC 10. NPE-ZRP coating was applied by the manufacturer according to their best practice and a traditional three-coat ZRP coating was applied by a 
specialized applicator over the same low carbon steel plate. Only a primer was tested for NPE-ZRP coating as provided by the manufacturer although the coating system can accommodate a topcoat for additional protection. After curing the coated plates were delivered to the laboratory facility for further evaluation.

Table 3.1. Chemical Composition of the Low Carbon Steel Panels.

\begin{tabular}{cccccc}
\hline \multicolumn{5}{c}{ Components (Max \%) } \\
\hline Carbon & Manganese & Phosphorus & Sulphur & Silicon & Copper \\
\hline 0.02 & 0.16 & 0.006 & 0.003 & 0.03 & 0.09 \\
\hline
\end{tabular}

\subsubsection{Initial Characterization}

Prior to testing, the test materials were characterized by their physical characteristics. Initial characterization of the samples in the as-received condition included coating thickness measurements, coating pull-off strength measurements, and identification of different coating features by optical microscopy. The coating thickness was measured from the average of multiple readings on the surface of the coated coupons. The coating thickness was measured using a DeFelsko Positector 6000 magnetic coating thickness gauge. Six readings were made on the surface of each test coupon to get the average. Pull-off strength measurements were made by using DeFelsko Positest manual pull-off adhesion tester following ASTM D4541-02. Metal dollies were glued to the surface of the coated coupon using a two-part epoxy adhesive and allowed to sit for 24 hours. The perimeter around the fastened dolly was then scored down to the steel substrate prior to testing with a pull-off adhesion tester. Metallographic preparation of samples for optical microscopy examination of coating cross-sections followed conventional methodologies. The grinding steps used $74 \mu \mathrm{m}, 20 \mu \mathrm{m}$, and $10 \mu \mathrm{m}$ 
abrasives, followed by polishing with $9 \mu \mathrm{m}, 3 \mu \mathrm{m}$, and $1 \mu \mathrm{m}$ diamond suspensions and $0.05 \mu \mathrm{m}$ silica suspensions. Then the samples were exposed for further evaluation.

\subsubsection{Long-term Exposure}

\subsubsection{Atmospheric Outdoor Exposure}

Samples were exposed to two outdoor test sites in South Florida confirming ASTM G7-11. The locations of the test sites are shown in Figure 3.1. Aluminum test racks approximately 10 feet in length and 5 feet in height were made available at both sites. The Beach Test Site at Tea Table Key in Islamorada, FL is situated immediately adjacent to the ocean with a strong presence of warm humid salty air. The ground cover was typically limestone rock. The Inland Test Site was located on the Florida International University engineering campus in Miami, FL located approximately 10 miles from the coast. The ground cover at the Inland Test Site was short grass. Coated steel sample coupons were placed on the test rack and oriented at $45^{\circ}$ to the horizon facing south. Weather conditions such as temperature, relative humidity, and rainfall were monitored during the exposure period and were comparable between the two test sites, as shown in Figure 3.2. To assess the effect of local coating damage, a $25.4 \mathrm{~mm}$ long and $0.5 \mathrm{~mm}$ wide scratch was introduced on one set of samples prior to testing. All the samples were visually evaluated after 4-month, 8-months, 12-months and 24-months of exposure. The amount of chloride deposition on the exposed samples was measured using a laboratory test kit. After 4 months, the chloride depositions at the inland and beach exposed samples were 0 , and $\sim 3 \mu \mathrm{S} / \mathrm{cm}^{2}$ respectively. Similar depositions were identified after 24 months of exposure. Chloride deposition was not accumulated on the exposed surface with time and likely due to the continuous washout due to precipitation 
at the outdoor environments. Part of the samples was collected after each selected exposure interval for the destructive examination of the coating degradation.

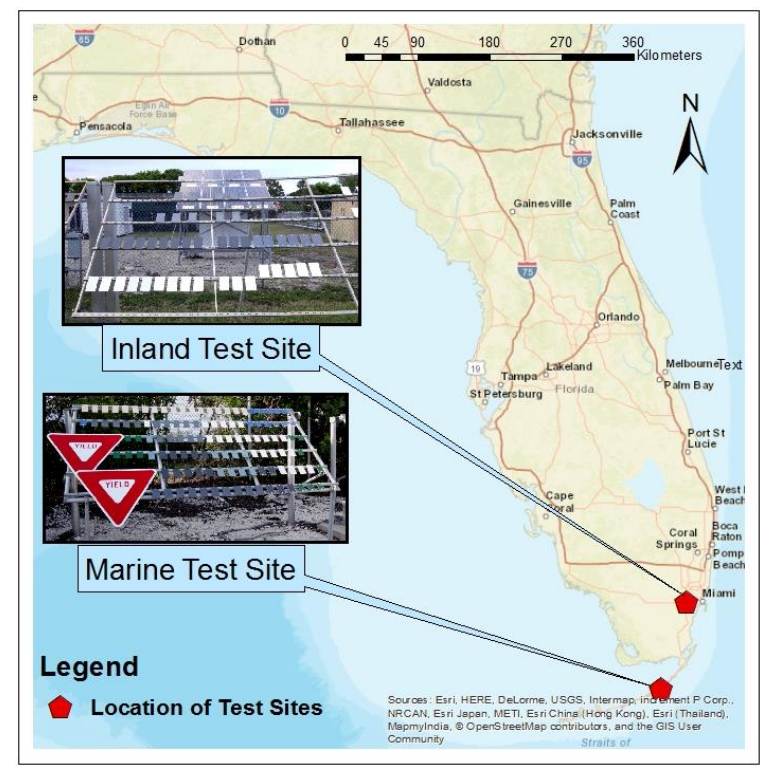

Figure 3.1. Location of Outdoor Exposure Sites (Sabbir, 2017).

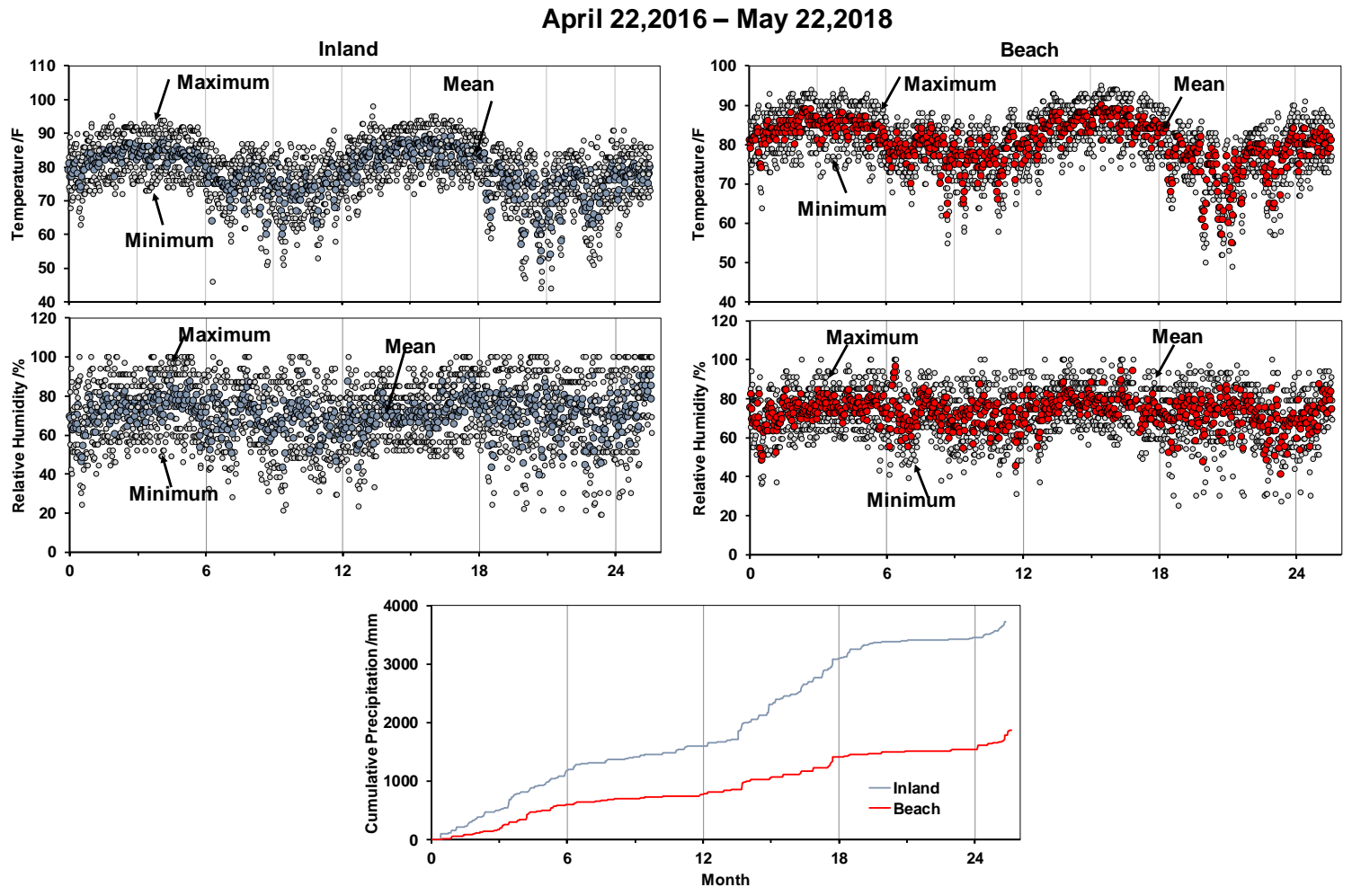

Figure 3.2. Weather Data for Outdoor Test Sites. 


\subsubsection{Aggressive Salt-Fog Exposure}

Samples were exposed to a salt-fog chamber conforming to ASTM B117-16 at Florida International University as shown in Figure 3.3. Tests included exposure in a saltfog chamber with the use of $5 \% \mathrm{NaCl}$ saturated salt solution for 24 months $(\sim 18,000$ hours) to evaluate the effects of aggressive exposure conditions on the integrity of the coating and corrosion of the steel coupons. The salt-fog chamber temperature was maintained at $\sim 32^{\circ} \mathrm{C}$. The samples were placed at $\sim 40^{\circ}$ inclination with support along the bottom edge of the coupon and along an edge at the upper third of the sample. Corrosion development was assessed by photo documentation over time. A set of test panels were removed at 4 months, 8 months, 12 months and 24 months ( $\sim 3600$ hours, $\sim 6000$ hours, $\sim 9000$ hours and $\sim 17,500$ hours) from environmental exposure to identify degradation of the coating by physical testing and other material testing. The amount of chloride deposition was measured using a laboratory test kit. After 4 months the chloride deposition at the salt-fog exposed samples was $50 \mu \mathrm{S} / \mathrm{cm}^{2}$. The similar deposition was also identified after 24 months of exposure.
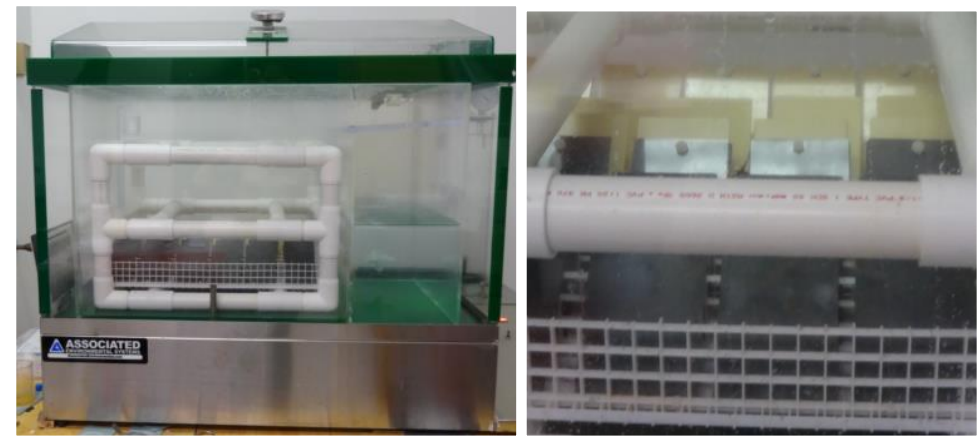

Figure 3.3. Test setup for Salt-fog Exposure. 


\subsubsection{Laboratory Electrochemical Testing}

\subsubsection{Water Immersion}

To understand the coating durability and corrosion development, a set of laboratory control as-received unscribed and scribed (defect) samples were immersed in neutral $\mathrm{pH}$ salt solution for 60 days to identify the possible effect of continuous exposure to the salt solution. To assess the effect of local coating damage, a $25.4 \mathrm{~mm}$ long and $0.5 \mathrm{~mm}$ wide straight scratch (deep enough to penetrate the coating to the underlying metal interface) was made. A $63.5 \mathrm{~mm}$ diameter acrylic cylinder pond, centered on the coated steel coupons, was filled with the test solution. Samples were exposed in $3.5 \mathrm{wt} \%$ (0.6 M) sodium chloride aqueous solution to simulate the runoff and pooled drainage water. The set up for electrochemical testing comprised of a three-electrode cell arrangement as shown in Figure 3.4. The coated steel coupon, working electrode had $\sim 126 \mathrm{~cm}^{2}$ of its surface in contact with the test solution. Activated titanium rods were used as counter and reference electrodes for each test cell (Castro et al., 1992), and the activated titanium reference electrode was calibrated with a saturated calomel electrode (SCE) during the test exposure. Electrochemical testing used a Gamry Reference 600 potentiostat and impedance analyzer as well as an ECM8 Multiplexer. Corrosion testing included Open Circuit Potential (OCP) measurements, Linear Polarization Resistance (LPR) and Electrochemical Impedance Spectroscopy (EIS). LPR measurements were made at various times from the initial OCP to $-25 \mathrm{mV}$, at a scan rate of $0.05 \mathrm{mV} / \mathrm{s}$. EIS testing was done at the OCP condition with $10 \mathrm{mV}$ AC perturbation voltage (Murray, 1997; El-Mahdy et al., 2000, \& Mahdavian \& Attar, 2006) from frequencies $100 \mathrm{kHz} \geq \mathrm{f} \geq$ $1 \mathrm{mHz}$ for evaluation of the corrosion mechanism considering the steel interface and from 
frequencies $1 \mathrm{MHz} \geq \mathrm{f} \geq 1 \mathrm{~Hz}$ for the evaluation of the coating layer property.

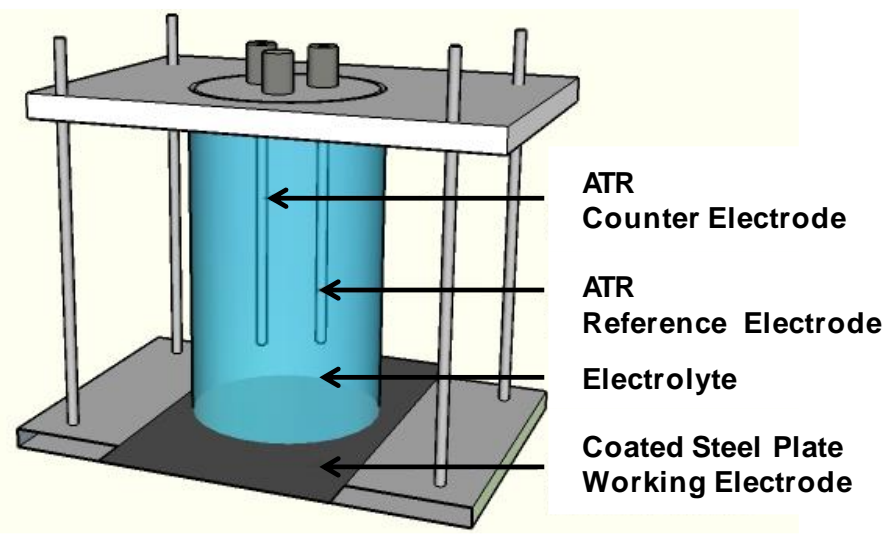

Figure 3.4. Test Cell Configuration.

\subsubsection{Electrochemical Polarization}

To identify the interaction of electrochemical activity of active coating components (zinc pigments) with exposed steel defects, the second set of laboratory samples were exposed at different polarization regimes, to discern electrochemical behavior of NPE-ZRP. A $3.2 \mathrm{~mm}$ diameter drill bit was used to introduce a coating defect ( $~ 0.75 \mathrm{~mm}$ nominal depth from the coating outer surface to the tip of the drill cavity) on the test coupon, exposing a $\sim 0.17 \mathrm{~cm}^{2}$ surface area of the steel substrate. The test setup of the polarization testing is as shown in Figure 3.5. Potentiostatic polarization levels included cathodic polarization regimes to identify the cathodic efficiency, open-circuit conditions to establish steady-state conditions, and an anodic polarization regime to assess the availability of zinc pigments to provide possible enhanced beneficial galvanic coupling. Polarization levels were made at $-1500 \mathrm{mV},-1100 \mathrm{mV},-900 \mathrm{mV},-600 \mathrm{mV}$, $300 \mathrm{mV}$, and $+100 \mathrm{mV}_{\mathrm{SCE}}$ for 30 days in $3.5 \%(\mathrm{w} / \mathrm{w}) \mathrm{NaCl}$ aqueous solution using a multipotentiostat. The polarization potentials were maintained within $\pm 10 \mathrm{mV}$ of the desired value. Currents readings were recorded during the polarized exposure period. 


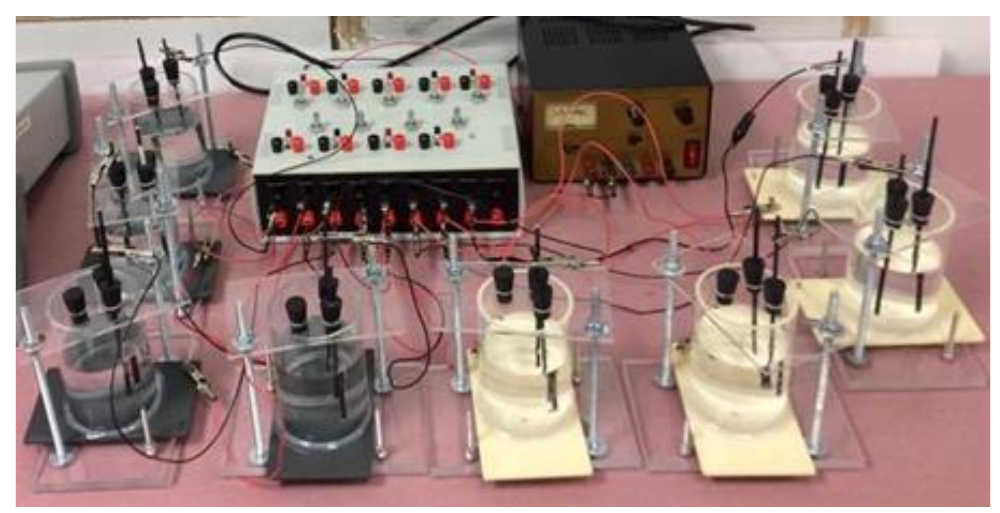

Figure 3.5. Laboratory Test Set-up for Potentiostatic Polarization.

\subsection{NPE-ZRP for Application of Repair Coating}

The nano-particle-enriched zinc-rich epoxy primer (NPE-ZRP) was tested to identify the durability for repair coating applications and if it could provide some benefits to overcome the field constraints of deficient surface preparation (surface contaminants as well as improper cleaning). Possible benefits could be attributed either from the enhanced galvanic coupling or mechanical bonding of the zinc pigments from the primer to the steel substrate. Since humid marine environments are expected to be a major factor during the surface preparation, moisture and salt contamination were incorporated before the repair coating application. A set of testing was made at outdoor atmospheric exposure and at alternate wet/dry cyclic exposure followed by immersing in $3.5 \mathrm{wt} \% \mathrm{NaCl}$ solution, exposure in salt-fog and exposure to the dry environment. Testing here considered localized coating damage that exposed the steel substrate. Furthermore, it was of interest to identify if nano-particles can extend the electrical connectivity of the embedded zinc pigments if the coating had improper surface preparation. In order to assess the effect of nano-particles on zinc electrochemical and mechanical coupling, steel corrosion development, the extent of zinc consumption, and coating degradation were addressed. A 
traditional zinc-rich primer (ZRP) was studied to reference the performance of nanoparticles.

\subsubsection{Sample Preparation}

Repair recommendations for traditional inorganic zinc-rich coating are complete removal of the existing coating (repainting) to repair with epoxy zinc or surface-tolerant epoxy coatings (spot or zone repair) (Francis, 2016). So, two forms of surface preparation were considered as sandblasting (SSPC 10) and hand cleaned (SSPC 3) to assess the repair durability. A conventional three-coat system was considered as the base coating system prior to the application of repair coating due to its predominant use on steel bridge girders since the 1980s. Sandblasting for repair applications would likely completely remove the base coating. Hand cleaning may leave remnant coating materials on the substrate prior to repair. Repair coating was applied to steel coupons with various substrate conditions. Surface cleanliness was varied by contaminating the surface with moisture and/or salt prior to repair the coating application. As repair coating systems are typically required to be applied shortly after surface cleaning to ensure proper bonding, the developed surface conditions prior to repair coating should allow discrimination of repair coating robustness for non-ideal surface preparation.

All tested samples ( 3 in $x 5$ in $x 1 / 8$ in) were cut from a plain carbon-steel sheet with chemical composition as shown in Table 3.1. A set of test panels were sandblasted to near white-surface conditions according to SSPC 10 by a professional coating's applicator, as shown in Figure 3.6. To minimize waste in the test sample preparation steps, uncoated steel plates were sandblasted in lieu of blast removal of a pre-coated three-coat system. The entire surface area of each coupon was prepared for repair coating 
application. After sandblasting, the panels were cleaned with a strong solvent (methyl ethyl ketone, MEK) and immediately stored in a controlled humidity chamber close to $0 \% \mathrm{RH}$ to prevent any flash rust formation.

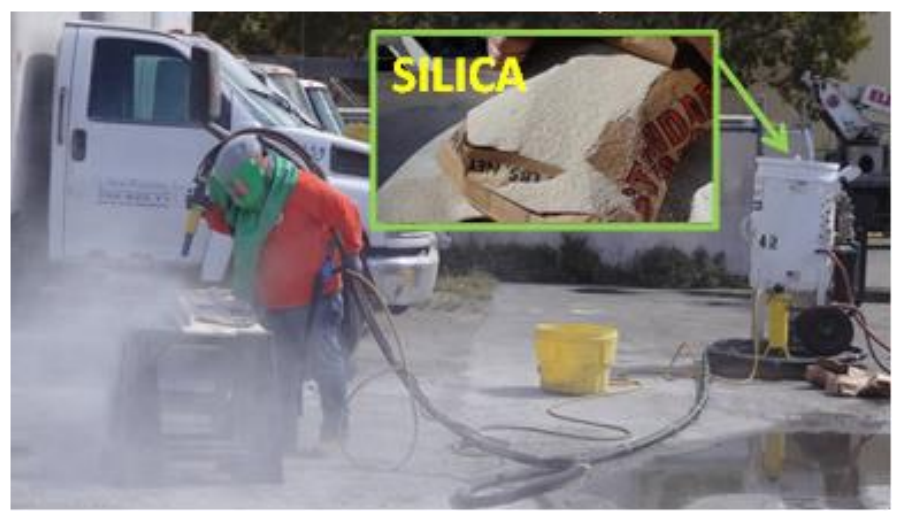

Figure 3.6. Sandblasting of the Coupons (Plain Steel Coupon).

A portable grinder was used to hand clean (surface abrade) another set of samples. The coated panels were ground with 60-grit sandpaper according to SSPC 3 as shown in Figure 3.7. The polyurethane topcoat and the epoxy midcoat were completely removed by the grinding process, but sporadic areas of zinc-rich primer were intentionally left, as shown in Figure 3.8. The panels were cleaned with a strong cleaning solvent (methyl ethyl ketone, MEK) and immediately stored in a controlled-humidity chamber close to $0 \% \mathrm{RH}$ to prevent any flash rust formation.

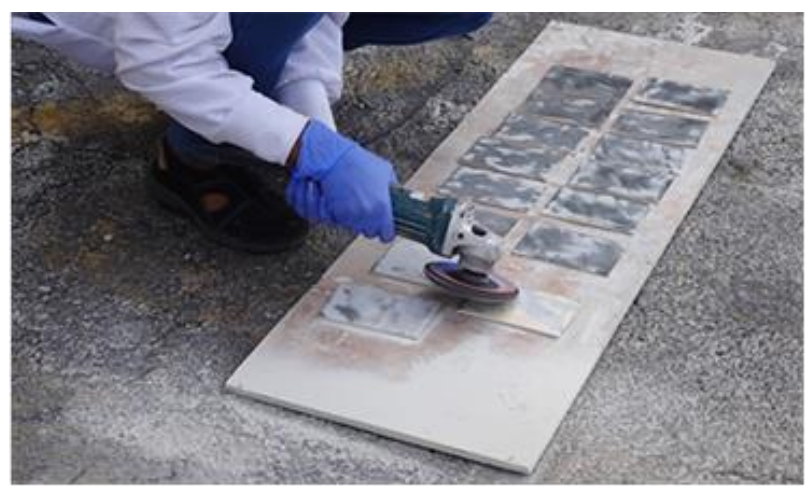

Figure 3.7. Surface Preparation by Grinding of Three-coat System. 


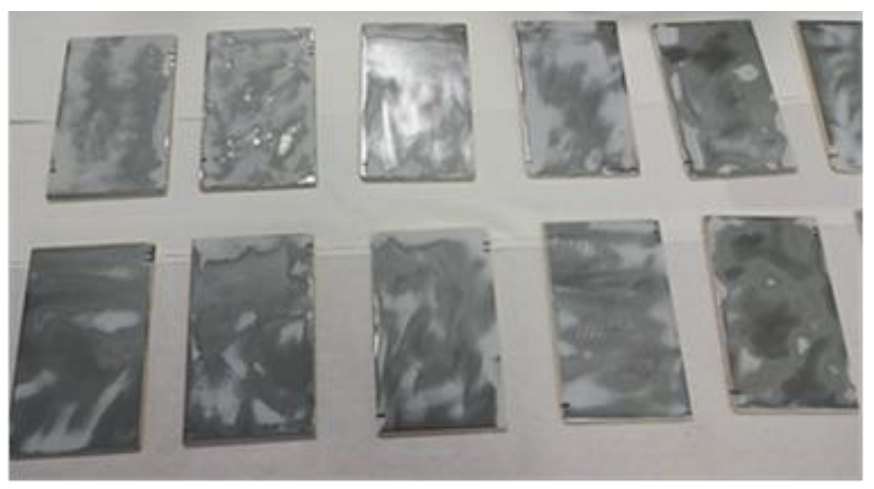

Figure 3.8. Hand Cleaned Steel Panels (Three-coat System).

In Florida marine environments, air-borne chlorides, salty sea mist, and yearlong high humidity create challenging conditions for coating repair applications. As part of the test setup, surface contaminations by exposure to moisture and chloride were considered. To simulate adverse exposure conditions before repair coating application, the panels were exposed to different levels of humidity $(5 \%, 75 \%$, and $100 \%$ or soaked in tap water) and surface salt contamination. Salt contamination was introduced within the first day of surface cleaning. A salt solution, $0.5 \mathrm{ml}$ of $3.5 \% \mathrm{NaCl}$, was placed on the sample surface $\left(\sim 15 \mathrm{in}^{2}\right)$ and then immediately dried with warm air to leave salt precipitates remaining on the surface. The expected surface chloride concentration was $\sim 82 \mu \mathrm{g} / \mathrm{cm}^{2}$. Results using a commercial test kit showed reproducibility of the surface chloride concentration between samples but with values $\sim 50 \mu \mathrm{g} / \mathrm{cm}^{2}$ as shown in Figure 3.9. Susceptibility to soluble salt varies with coating type, thickness, and exposure environment. Even though, the measured $\sim 50 \mu \mathrm{g} / \mathrm{cm}^{2} \mathrm{Cl}^{-}$of salt concentration was considered to be borderline marginal for coating degradation, the surface rust formation provides a condition to assess coating robustness. These surface chloride levels were thought to be sufficient to induce some level of coating distress but not necessarily overly aggressive. 


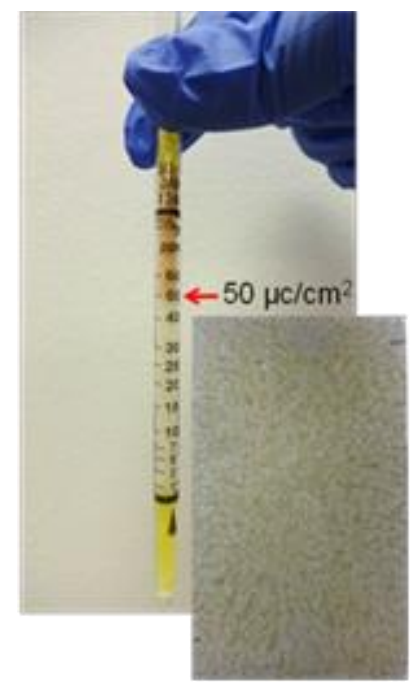

Figure 3.9. Measurement of the Chloride Contamination Level.

To expose the panels to different levels of humidity, the test panels were sealed in controlled-humidity glass chambers, as shown in Figure 3.10. All samples had approximately 3 days of environmental preconditioning after surface cleaning ( 1-day storage in $<5 \% \mathrm{RH}$ and 2 days of exposure in $5-100 \% \mathrm{RH}$ ) and before application of the coating, as summarized in Table 3.2. A desiccator was used to maintain low relative humidity less than 5\%RH. Saturated sodium chloride solution and deionized water were used in a sealed humidity chamber to maintain $75 \%$ and $100 \%$ relative humidity conditions, respectively at room temperature.

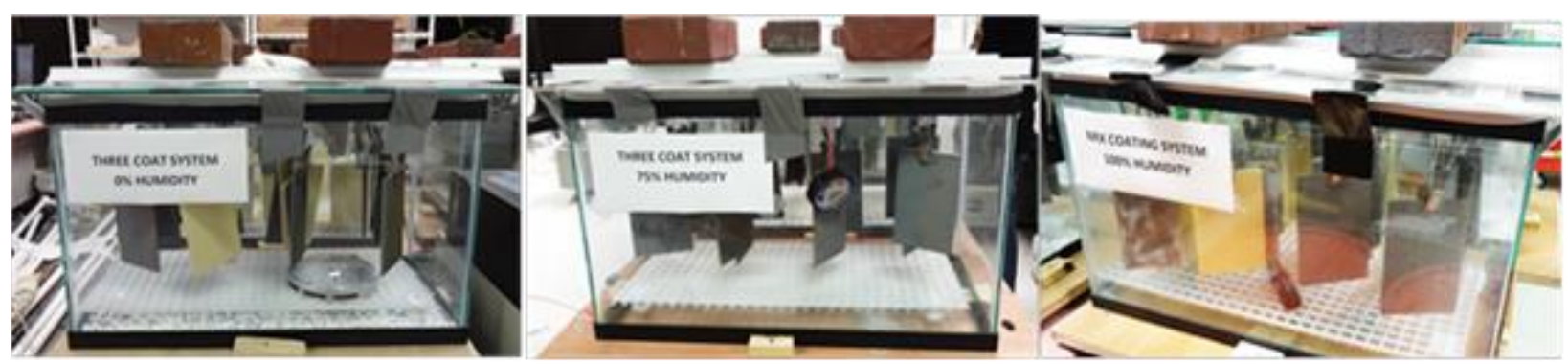

Figure 3.10. Humidity Chamber for Test Panel Exposure. 
Table 3.2. Pre-coating Surface Preparation.

\begin{tabular}{|c|c|c|c|}
\hline Coating Type & $\begin{array}{l}\text { Surface } \\
\text { Cleaning }\end{array}$ & $\begin{array}{l}\text { Chloride } \\
\left(\mu \mathrm{g} / \mathrm{cm}^{2}\right)\end{array}$ & $\begin{array}{c}\text { Humidity Pre- } \\
\text { Exposure (\% RH) }\end{array}$ \\
\hline \multirow{2}{*}{ ZRP } & \multirow{2}{*}{ Sandblast ${ }^{2}$} & 0 & $5,75,100$, Soaked \\
\hline & & $82^{4}, 50^{5}$ & 5,75 \\
\hline \multirow{4}{*}{ NPE-ZRP } & \multirow{2}{*}{ Sandblast ${ }^{2}$} & 0 & $5,75,100$ \\
\hline & & $82^{4}, 50^{5}$ & 5,75 \\
\hline & \multirow{2}{*}{$\begin{array}{c}\text { Surface } \\
\text { Abrasion } \\
60 \text { Grit }^{3}\end{array}$} & 0 & $5,75,100$, Soaked \\
\hline & & $82^{4}, 50^{5}$ & 5,75 \\
\hline
\end{tabular}

1. $\sim 2$ day RH preconditioning prior to coating application. 2. SSPC10.

3. SSPC3. 4. Estimated concentration. 5. Measured concentration.

After exposure to adverse conditioning, repair coatings were applied over the steel panels. Coatings were applied under similar application conditions of temperature $\left(74^{\circ} \mathrm{F}\right)$ and humidity ( $\sim 80 \% \mathrm{RH})$ using standard equipment that is recommended by the manufacturer. The coated plates were then delivered to the laboratory facility for further evaluation. Before exposure for robustness evaluation coated samples were initially characterized to have base cases for comparison. In outdoor exposure infrastructure corrosion is influenced by many factors, as corrosive atmospheres, temperature, rain, and wet/dry cyclic exposure. These factors usually have a synergistic effect on each other. Hence, cyclic testing was proposed to provide a more representative simulation of the interaction of these factors than is found in traditional tests with continuous exposure to a static exposure. Another set of samples were also exposed to outdoor atmospheric exposure. Figure 3.11 displays the flow chart of repair coating exposure evaluation. 


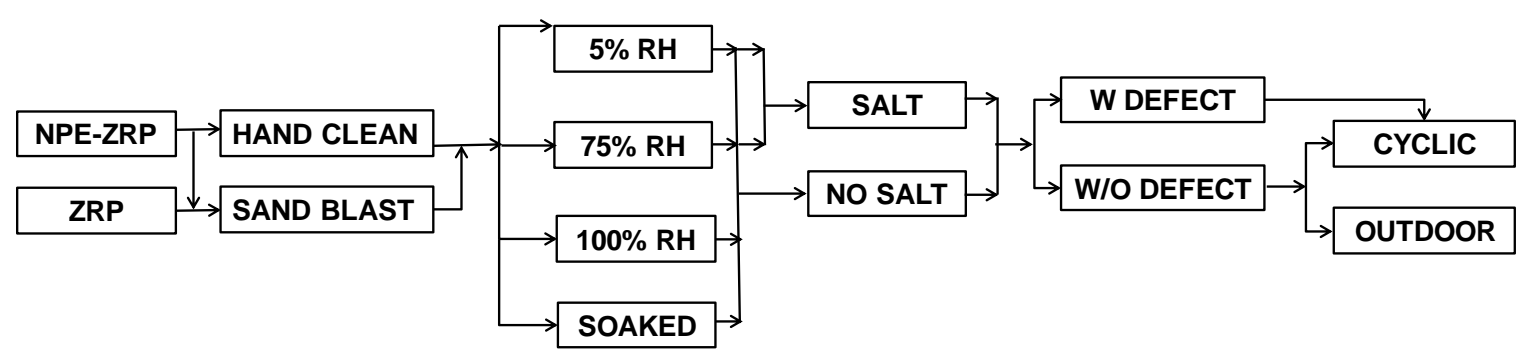

Figure 3.11. Sample Exposure after Repair.

\subsubsection{Initial Characterization}

Initial characterization of the samples after repair coating application in the asreceived condition included coating thickness measurements, coating pull-off strength measurements, and identification of different coating features by optical microscopy. The coating thickness was measured from the average of multiple readings on the surface of the coated coupons. The coating thickness was measured using a DeFelsko Positector 6000 magnetic coating thickness gauge. Six readings were made on the surface of each test coupon to get the average. Pull-off strength measurements were made by using DeFelsko Positest manual pull-off adhesion tester following ASTM D4541-02. Metal dollies were glued to the surface of the coated coupon using a two-part epoxy adhesive and allowed to sit for 24 hours. The perimeter around the fastened dolly was then scored down to the steel substrate prior to testing with a pull-off adhesion tester. Metallographic preparation of samples for optical microscopy examination of coating cross-sections followed conventional methodologies. The grinding steps used $74 \mu \mathrm{m}, 20 \mu \mathrm{m}$, and $10 \mu \mathrm{m}$ abrasives, followed by polishing with $9-\mu \mathrm{m}, 3-\mu \mathrm{m}$, and $1-\mu \mathrm{m}$ diamond suspensions and $0.05 \mu \mathrm{m}$ silica suspensions. 


\subsubsection{Outdoor Testing}

A set of repair coupons were exposed at an outdoor inland test site on July 10, 2017, in South Florida, 16 km (10 miles) from the coast. Subsequent evaluations were made after 4 and 8 months. The coated coupons were exposed at $45^{\circ}$ facing south according to ASTM G 7M. The outdoor test site during the exposure is shown in Figure 3.12. Weather conditions such as temperature, relative humidity, and rainfall were monitored during the time of testing as shown in Figure 3.13.

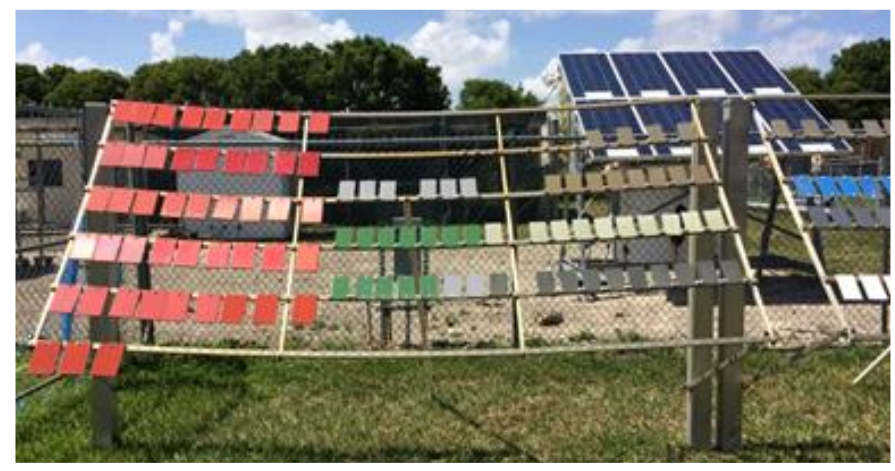

Figure 3.12. Outdoor Sample Exposure at FIU.

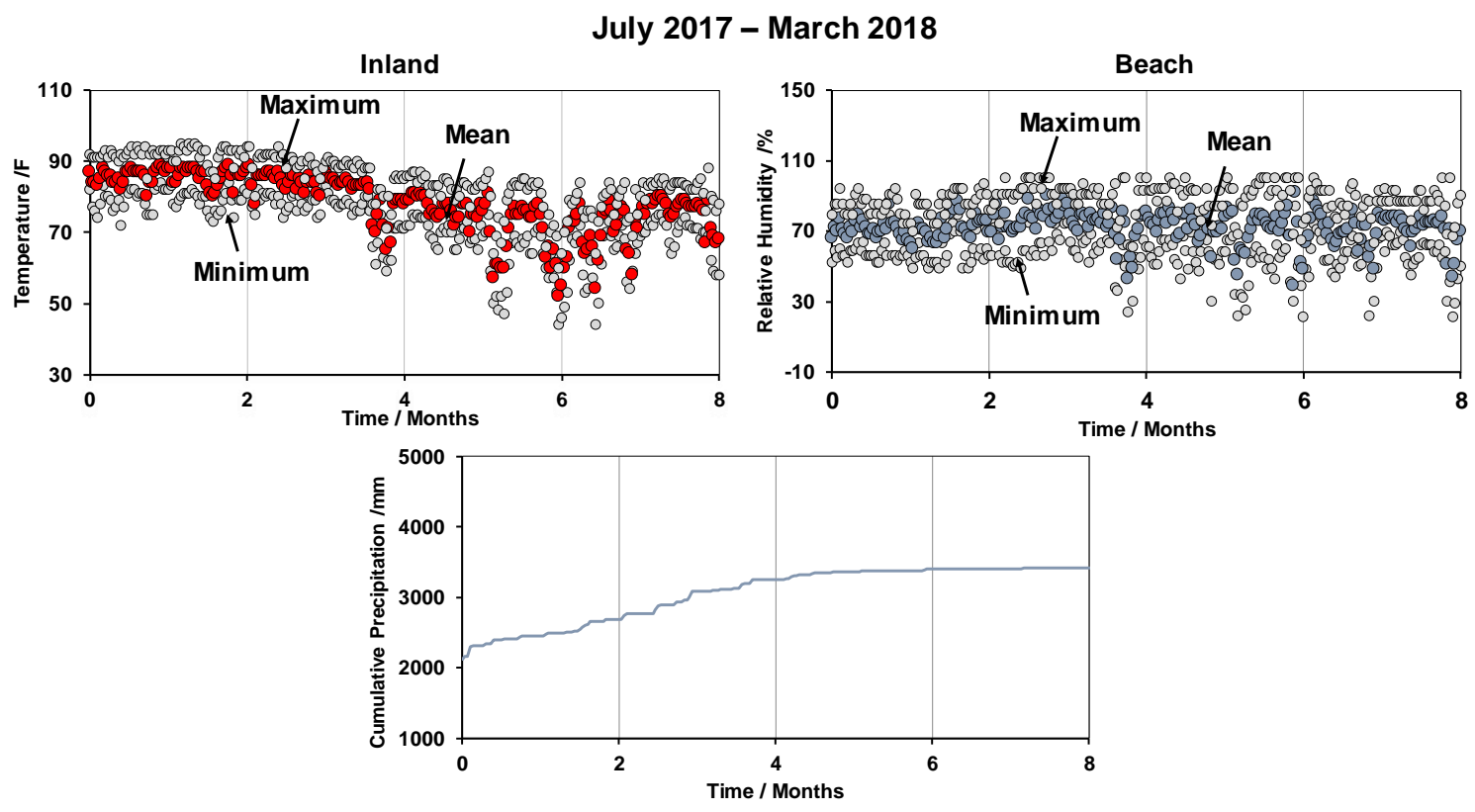

Figure 3.13. Weather Data for FIU Test Site. 


\subsubsection{Cyclic Wet/Dry Exposure}

A set of the coated samples were exposed to cyclic alternate wet/dry conditions. Samples were divided into two subgroups. One subgroup had an extended conical defect formed on the center of the coupon, exposing the steel substrate below the coating. The second subgroup did not have any surface coating damage. For the former, a $3.2 \mathrm{~mm}$ diameter drill bit was used to introduce the coating defect with a nominal depth from the coating outer surface to the tip of the drill cavity of $\sim 1 \mathrm{~mm}$. A cycle lasted 7 days and consisted of a sequence of exposures including 2 days of saltwater immersion (3.5 wt $\%$ $\mathrm{NaCl}$ ), 3 days of drying, and 2 days of salt-fog exposure (following ASTM B117) as represented in Table 3.3 and shown in Figure 3.14. A total of 20 cycles were made. The 140 days of exposure was not meant to simulate actual field exposure conditions but rather to provide early indicators of coating performance with the effects of the coatings with non-ideal surface preparation exposed to aggravating wet/dry salt exposure.

Table 3.3. Alternate Wet/Dry Exposure Sequence (20 Cycles).

\begin{tabular}{ccc}
\hline Exposure Sequence & Exposure Time & Criteria \\
\hline 1. Chloride Solution Immersion & 2 Days & $3.5 \mathrm{wt} \% \mathrm{NaCl}, 22-25^{\circ} \mathrm{C}$ \\
\hline \multirow{2}{*}{ 2. Drying } & 1 Day & $\mathrm{Ambient} \mathrm{RH} \mathrm{\&} \mathrm{T}^{1}$ \\
\cline { 2 - 3 } & 2 Days & $\sim 20 \% \mathrm{RH}, 22-25^{\circ} \mathrm{C}$ \\
\hline 3. Salt-Fog & 2 Days & $5 \% \mathrm{wt} \% \mathrm{NaCl}^{2}, \sim 35^{\circ} \mathrm{C}^{2}$ \\
\hline
\end{tabular}

1. Ambient air-conditioned laboratory environment. 2. ASTM B117

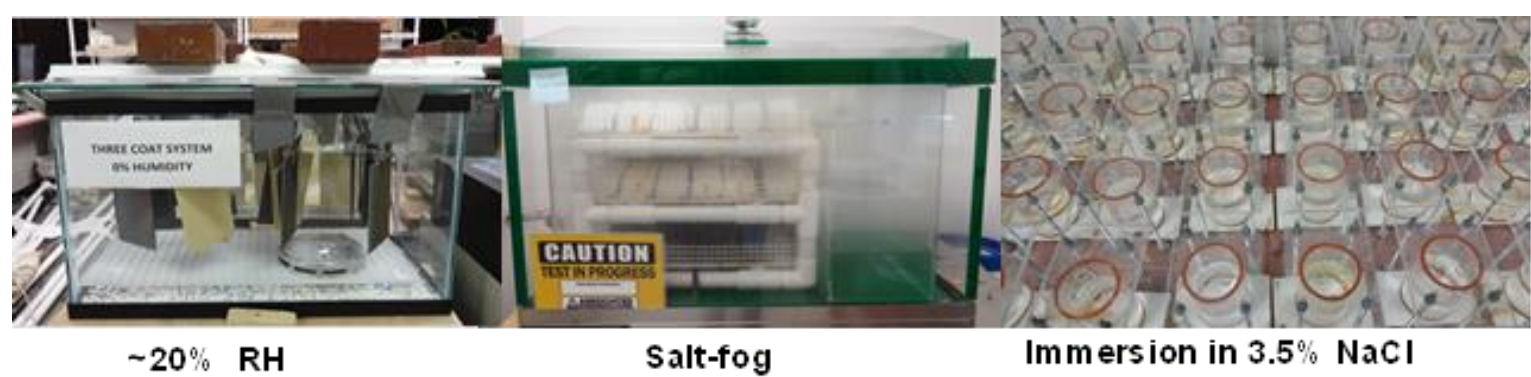

Figure 3.14. Cyclic Wet/Dry Exposure in Three Different Condition (20 Cycles). 
Electrochemical tests were made during the water immersion phase of the exposure sequence. These comprised of open circuit potential (OCP) measurements, linear polarization resistance (LPR). The test set-up had a three-electrode cell arrangement. A 2.5-inch diameter acrylic cylinder was centered on the coated steel coupons to create a pond for the addition of a $3.5 \%$ wt $\mathrm{NaCl}$ solution. The coated steel coupon was the working electrode. The surface area of the coupon surface in contact with the solution was $126 \mathrm{~cm}^{2}$. An activated titanium rod was used as a counter and reference electrode for each test cell for electrochemical testing. The activated titanium reference electrode was calibrated with a saturated calomel electrode (SCE). LPR testing was done from the initial OCP to $-25 \mathrm{mV}$ vs. OCP at a scan rate of $0.05 \mathrm{mV} / \mathrm{s}$.

\subsection{Material Evaluation}

The assessment of coating material durability and protection mechanism, in terms of different exposure environments, were considered in this research. For repair consideration, inappropriate surface conditioning was incorporated. To achieve the objective, measurement of coating thickness change, coating adhesion loss by knife test and pull-off strength with time after exposure were done. The coating thickness variation and pull-off strength measurement can be used to evaluate coating deterioration with exposure duration (Mittal, 1983). Eventual change of this property will give an indication of the coating deterioration mode and their propagation with time. Optical microscopy, as well as XRD, SEM and EDS, can also be conducted to inspect the composition of formed material by exposure.

After exposure, qualitative visual comparisons of all exposed samples were done to assess the coating condition and degree of corrosion. Assessment of coating thickness 
before and after exposure of each testing environment was carried out to verify the visual observation.

Coating thickness change and coating pull-off strength were measured as described earlier. The reported coating failure mode was considered by the priority ranking shown in Figure 3.15, where the primer was considered the major coating component to provide protection to the steel substrate.

\begin{tabular}{|l|l|}
\hline Topcoat & \\
\hline Midcoat & \\
\hline Primer & \\
\hline Mill Scale & \\
\hline
\end{tabular}

Figure 3.15. Coating Separation Modality.

Pull-off testing results were categorized as a primer cohesive failure when both the dolly and the coupon retained the primer. The failure was considered as topcoat adhesive failure when the dolly had the topcoat material and the coupon retained the midcoat/primer material. The failure was considered as a midcoat cohesive failure when both the dolly and the coupon retained the midcoat and midcoat adhesive failure was defined as when the dolly retained the midcoat material and the coupon retained primer. The failure was considered a primer cohesive failure when both the dolly and the coupon retained the primer. The failure was considered a primer adhesive failure when the dolly had the silver-colored primer and the coupon showed some level of grey that could be from the steel substrate, remnant mill scale, or initial surface rust. 
The influence of inappropriate surface preparation on coating bond strength also tried to evaluate by the surface measurement. A goniometer (from FAMAS) was used to measure the wetting properties (i. e., contact angle, surface energy and work of adhesion) by static sessile drop method. In each sample, five contact angle measurements were performed. Wetting liquids were deionized water, ethylene glycol, and diiodmethane. The drop volume was $\sim 5 \mu \mathrm{l}$ for deionized water, ethylene glycol and $\sim 2 \mu \mathrm{l}$ for diiodmethane. The syringe temperature was $20^{\circ} \mathrm{C}$ and the relative humidity was $50 \%$.

Coating failure was also evaluated from disbonded radial length (from the outer perimeter of the defect) by radial cuts. $30^{\circ}$ radial scratch angle were made by means of a utility knife around the defect diameter for adhesion loss determination. An Exacto knife was used afterward to check for disbonded radial length. Cross-sectional micrograph of the exposed samples for microscopic evaluation was followed by conventional grinding and polishing steps as described earlier. Comparative evaluation of exposed samples at different exposure conditions with the as-received condition provided an indication of coating deterioration level as well as coating interfaces.

X-Ray diffraction was done by using a Diffraktometer D5000 with the help of Diffrac Plus data acquisition software. Coated steel coupon sections, exposed coating, as well as corrosion products, was evaluated to identify material deterioration. A silicon substrate was used to reduce the background noise. As-received and exposed test samples were selected for comparative evaluation. The testing procedure included diffraction scans from 5 to 70 degrees with a $2^{\circ} /$ minute scan rate. Peak normalization, subtraction of the background and integration were done using Origin Lab 7.5 software. The database PDF 4 was used to identify any crystalline material that formed during the exposure. 
Scanning electron microscopy (SEM) and energy dispersive spectroscopy (EDS) were used for coating material characterization. A JEOL 6330F SEM with Energydispersive X-ray spectroscopy analytical software (Noran System Six) was used for the analysis. To make the nonconductive epoxy coating conductive, samples were coated with gold for $55 \mathrm{~s}$ that built a $5 \mathrm{~nm}$ thick gold layer over the sample. A carbon tape was attached to the steel face to the epoxy mounted coating samples to maintain the connectivity. $38 \mathrm{~mm}$ working distance was used for EDS analysis. An accelerating voltage of $\sim 25 \mathrm{kV}$ was used for imaging. From the SEM images and EDS spectrum, the coating microstructure along with elemental identification was assessed for the supporting evidence of coating deterioration. 


\section{CHAPTER 4}

\section{PERFORMANCE OF THE NPE-ZRP COATING SYSTEM}

\subsection{Introduction}

The performance of the NPE-ZRP coating system was evaluated for the application of new highway steel bridge structures if it could provide enhanced coupling of the zinc pigments from the primer to the steel substrate. Testing incorporated atmospheric exposures with various moisture and salt environments including extended outdoor atmospheric and salt-fog exposures. Since moisture and salt were expected to be a major factor in the marine environment, testing also included immersion of coated steel samples in $3.5 \mathrm{wt} \% \mathrm{NaCl}$ solution. Testing considered localized coating damage that exposed the steel substrate. Test conditions to promote the electrochemical activity of the zinc (including both oxidation and reduction reactions) included various levels of electrochemical polarization. It was of interest to identify if nano-particles can extend the connectivity of embedded zinc pigments. In order to assess the effect of nano-particles on zinc electrochemical coupling, steel corrosion development, the extent of zinc consumption, and coating degradation were addressed. A traditional zinc-rich primer (ZRP) was studied to reference the performance of nano-particles.

\subsection{Material Characterization}

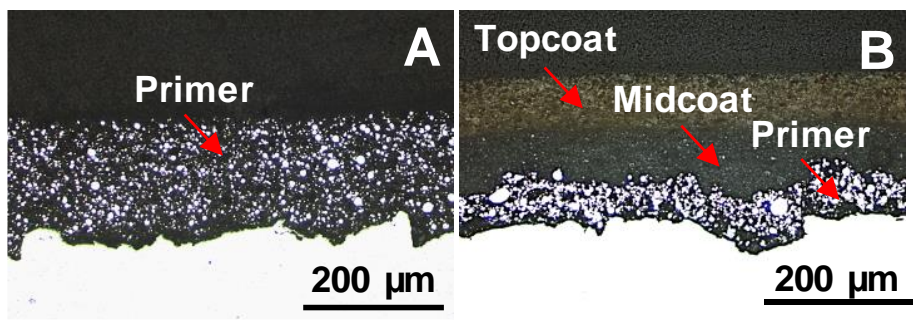

Figure 4.1. Metallographic Cross-sections of Test Coatings. A) NPE-ZRP Coating and B) ZRP Coating 
NPE-ZRP and ZRP coatings were applied over A36 carbon steel coupons (76.2 $\mathrm{mm} \times 127 \mathrm{~mm} \times 3.2 \mathrm{~mm}$ ) following the manufacturer requirements. Coatings characteristics are summarized in Table 4.1 and their metallographic cross-sections in the as-received conditions are shown in Figure 4.1. In NPE-ZRP, the zinc pigments were more broadly dispersed in the epoxy matrix in comparison to ZRP. Figure 4.2 represents the particle size distribution for both coatings throughout the cross-section of the primer. As seen in the figure the ZRP contains a comparatively well-distributed size of zinc pigments whereas NPE-ZRP contains more concentration of small size pigments the primer matrix. The density of the zinc pigment presence was approximately half $(\sim 25 \%$ and $\sim 35 \%$ cross-sectional zinc area for NPE-ZRP and ZRP respectively) in comparison to the ZRP. Aside from the difference in zinc pigment dispersion, from a cross-sectional view, the total zinc pigment area in the NPE-ZRP primer was similar for the ZRP primer.
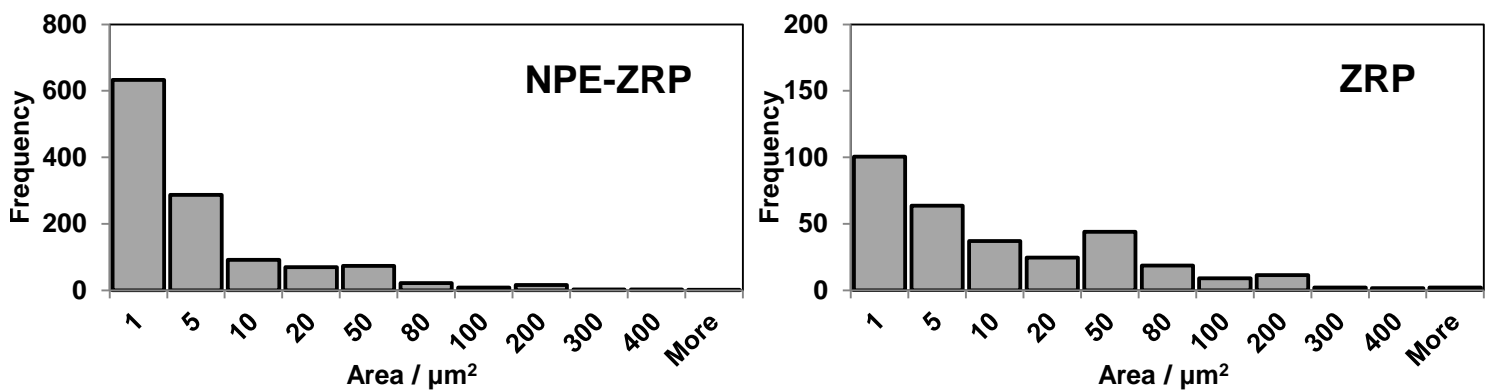

Figure 4.2. Zinc Particle Size Distribution in the Polymer Matrix. 
Table 4.1. Characteristics of the Coatings under Study

\begin{tabular}{|c|c|c|c|c|}
\hline $\begin{array}{c}\text { COATING } \\
\text { TYPE }\end{array}$ & \multicolumn{4}{|c|}{ CHARACTERISTICS } \\
\hline \multirow{6}{*}{ NPE-ZRP } & \multicolumn{4}{|l|}{ Coating System } \\
\hline & Primer & Epoxy Binder & $\begin{array}{l}\text { (Zinc pigments with } \\
\text { carbon nano-particles) }\end{array}$ & $\sim 75 \% \mathrm{Zn}$ \\
\hline & Midcoat & - & & \\
\hline & Topcoat & - & & \\
\hline & Total Thickness & $\sim 250 \mu \mathrm{m}$ & & \\
\hline & Pull-off Strength & $>10,000 \mathrm{kPa} *$ & & \\
\hline \multirow{6}{*}{ ZRP } & Coating System & & & \\
\hline & Primer & Silicate Binder & (Zinc pigments) & $\sim 85 \% \mathrm{Zn}$ \\
\hline & Midcoat & Epoxy & & \\
\hline & Topcoat & Urethane & & \\
\hline & Total Thickness & $\sim 420 \mu \mathrm{m}$ & Primer $\sim 100 \mu \mathrm{m}$ & \\
\hline & Pull-off Strength & $\sim 5,000 \mathrm{kPa} * *$ & & \\
\hline
\end{tabular}

* Value represents minimum bound due to test failure mode (glue failure).

**Average value.

Coating thicknesses were measured, using a magnetic thickness gauge and verified by optical microscopy of the cross-section of the sample. The average thickness for NPE-ZRP and ZRP test samples was $\sim 250 \mu \mathrm{m}$ and $\sim 420 \mu \mathrm{m}$, respectively. Pull-off strengths were measured by means of a pull-off strength adhesion tester and the average strength was $>10,000 \mathrm{kPa}$ for NPE-ZRP and $\sim 5,000 \mathrm{kPa}$ for ZRP in the as-received condition. Pull-off testing for NPE-ZRP coated samples mostly failed due to glue failure whereas for ZRP samples failed mostly due to topcoat adhesive and for some cases by primer cohesive failure. A representative picture of different failure modality is shown in Figure 4.3. 

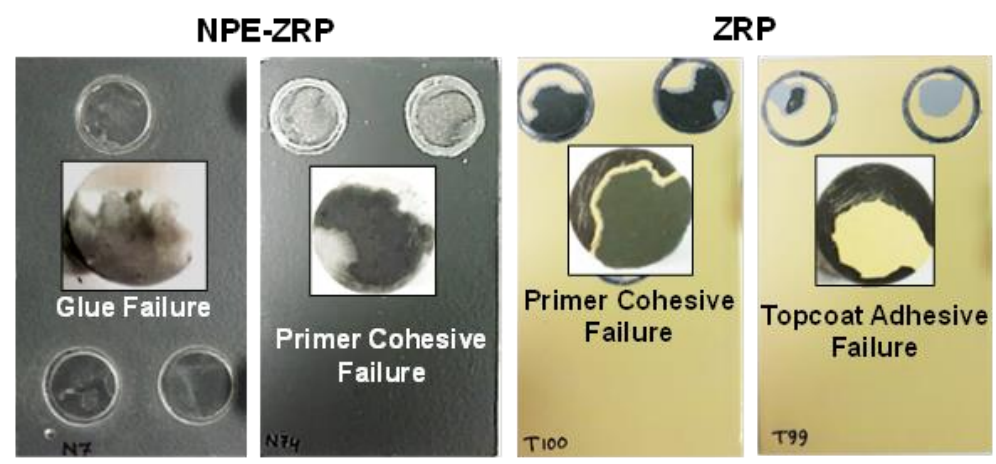

Figure 4.3. Different Coating Failure Modality.

\subsection{Results and Discussion}

\subsubsection{Atmospheric Outdoor and Salt-Fog Exposure}

\subsubsection{Visual Observation}

Photographic appearances of the coated samples for both NPE-ZRP and ZRP coatings after extended 24 months of atmospheric exposure are shown in Figure 4.4 (periodic pictures with exposure time are presented in Appendix A). A similar performance was observed between the samples of the inland and beach test sites for both coatings. There was no significant visual coating deterioration observed after 24 months of exposure, but the surface color change observed was likely due to polymer degradation for both coatings. Surface discoloration started within 1 month of exposure and progressively deteriorated over the exposure period. White staining (white rust) was observed over the NPE-ZRP coupon surface due to the oxidation of exposed zinc pigments, as there was no topcoat. For both coatings, tarnishing of the exposed steel at the scribe region started initially within 15 days of exposure and by the time of sample extraction after 24 months of exposure, the defect sites contained accumulated corrosion product. Similar coating degradation and rust bleeding at the defect sites were observed for both NPE-ZRP and ZRP exposed samples for up to 24 months is salt-fog exposure. 


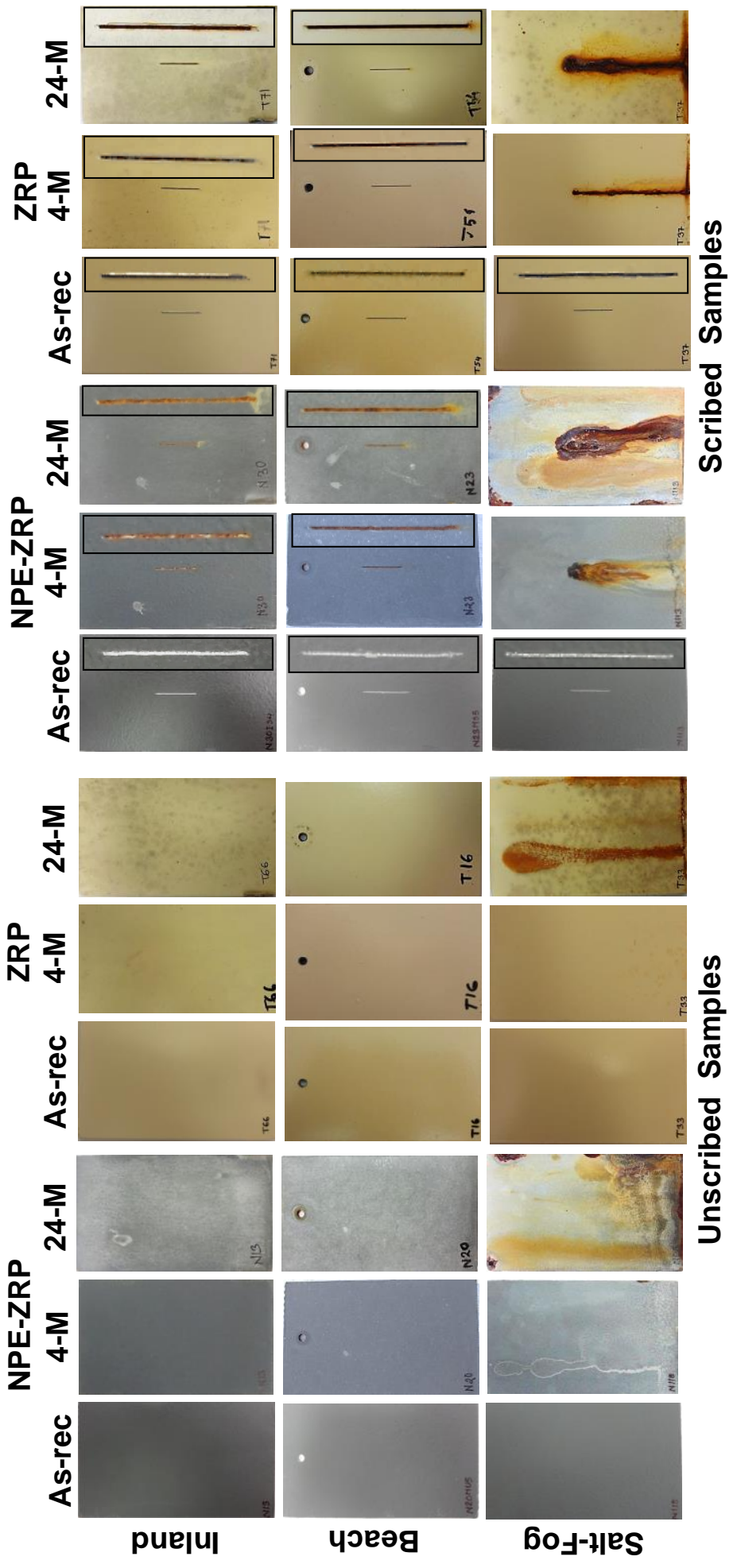

Figure 4.4. Visual Appearance of the Outdoors and Salt-fog Exposed Samples. 


\subsubsection{Coating Thickness}

Degradation of both coatings from outdoor and salt-fog exposures was assessed by coating thickness measurements after 4, 8, 12 and 24 months of exposure (Figure 4.5). The changes in coating thickness are represented with the time of exposure for different exposure conditions. For inland test samples of both coatings, there was apparently no change in coating thickness, whereas for beach test samples there was a gradual decrease and again increase in coating thickness. The decrease in thickness of the coating layer was apparently due to the polymer degradation by photochemical reaction due to environmental attack (light, heat, moisture, and oxygen) and the increment in thickness was mostly associated with the zinc corrosion product (which was reflected in visual observation by tarnishing and white spot over the coating surface) as zinc corrosion products occupy a larger volume than the native zinc pigment (Porter, 1994). In salt-fog exposure, coating thickness increased significantly $(\sim 40 \mu \mathrm{m})$ up to $\sim 4$ months apparently associated with the increment in volume of zinc corrosion product and afterward for further exposure up to 24 months the increment was gradual in a slower rate for NPEZRP coating; whereas, for ZRP coating the increment in thickness was gradual with a comparatively slower rate. The large increment in the thickness of NPE-ZRP coating was apparently associated with the oxidation of available zinc pigments from the exposed top surface. For ZRP coating it was comparatively less because of additional coverage of zinc pigments in the primer layer from the above two layers (midcoat $\&$ topcoat). 

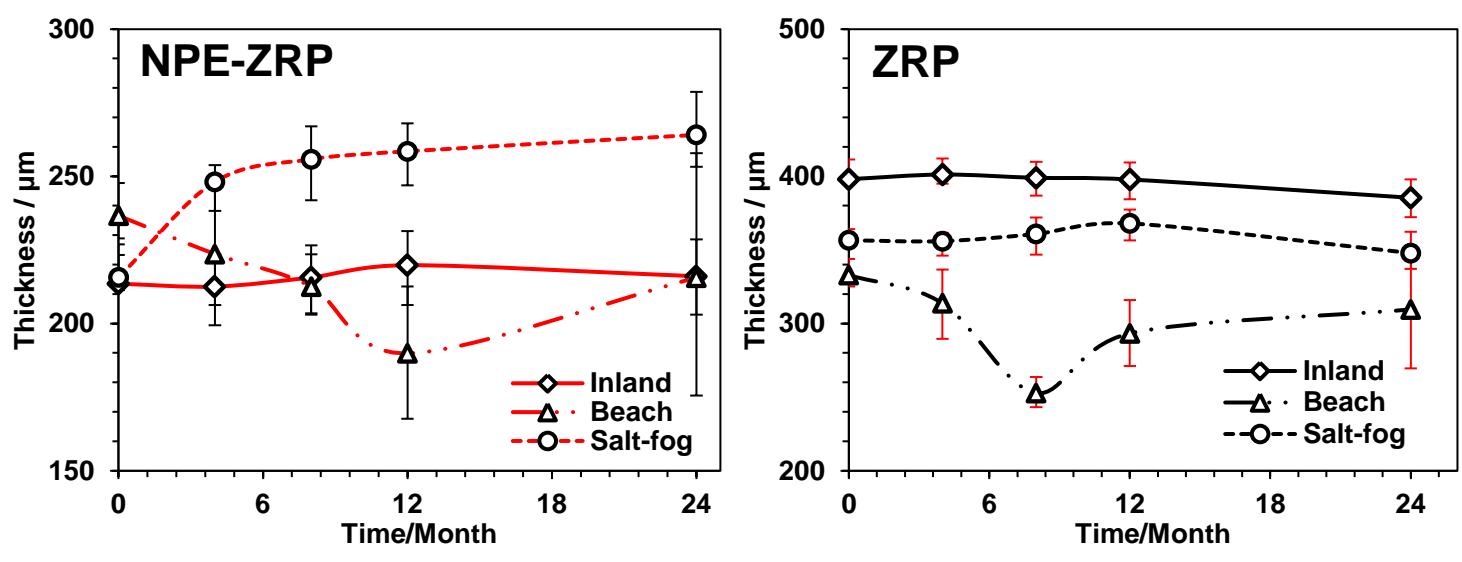

Figure 4.5. Coating Thickness of Outdoor and Salt-fog Exposed Samples over Time.

\subsubsection{Pull-off Strength}

Pull-off strength results for both NPE-ZRP and ZRP coatings with time in different exposure environments are shown in Figure 4.6. After both outdoor (inland and beach) exposure, NPE-ZRP coating pull-off testing generally resulted in glue failures which alone can only provide a minimum bound for coating pull-off strength. Detail of failure modality is shown in Appendix B. The test results for ZRP either resulted in topcoat adhesive failure or primer cohesive failure with values ranging from $\sim 3,000$ $11,000 \mathrm{kPa}$. For the inland and early exposure at the beach site (up to 8 months), the minimum bound pull-off strength for NPE-ZRP was generally higher than the actual pulloff strength for ZRP, indicating better mechanical behavior for NPE-ZRP relative to ZRP. However, for longer exposures at the beach site and in salt-fog testing, the testing did not provide a clear indication of performance due to the generally low minimum bound pull-off strength (glue failure) of NPE-ZRP compared to the higher actual pull-off strength (coating failure) for ZRP. So, after exposure, pull-off testing of outdoor samples for NPE-ZRP coating did not result in adhesive or cohesive failures implicating the overall integrity of the primer coating. However, the coating pull-off test after exposure 
gave some indication of minor coating degradation. The mechanical dolly after testing typically showed evidence of residual particles that appeared to be removed from the primer substrate. The residual particles were thought to be the surface zinc oxidation product deposited on the primer surface described earlier. The result of salt-fog samples indicated possible degradation of NPE-ZRP in extended exposures in moist salt environments. However, the surface appearances of NPE-ZRP with salt-fog exposure up to 24 months were similar to comparable ZRP samples and did not show a propensity for worse coating degradation.

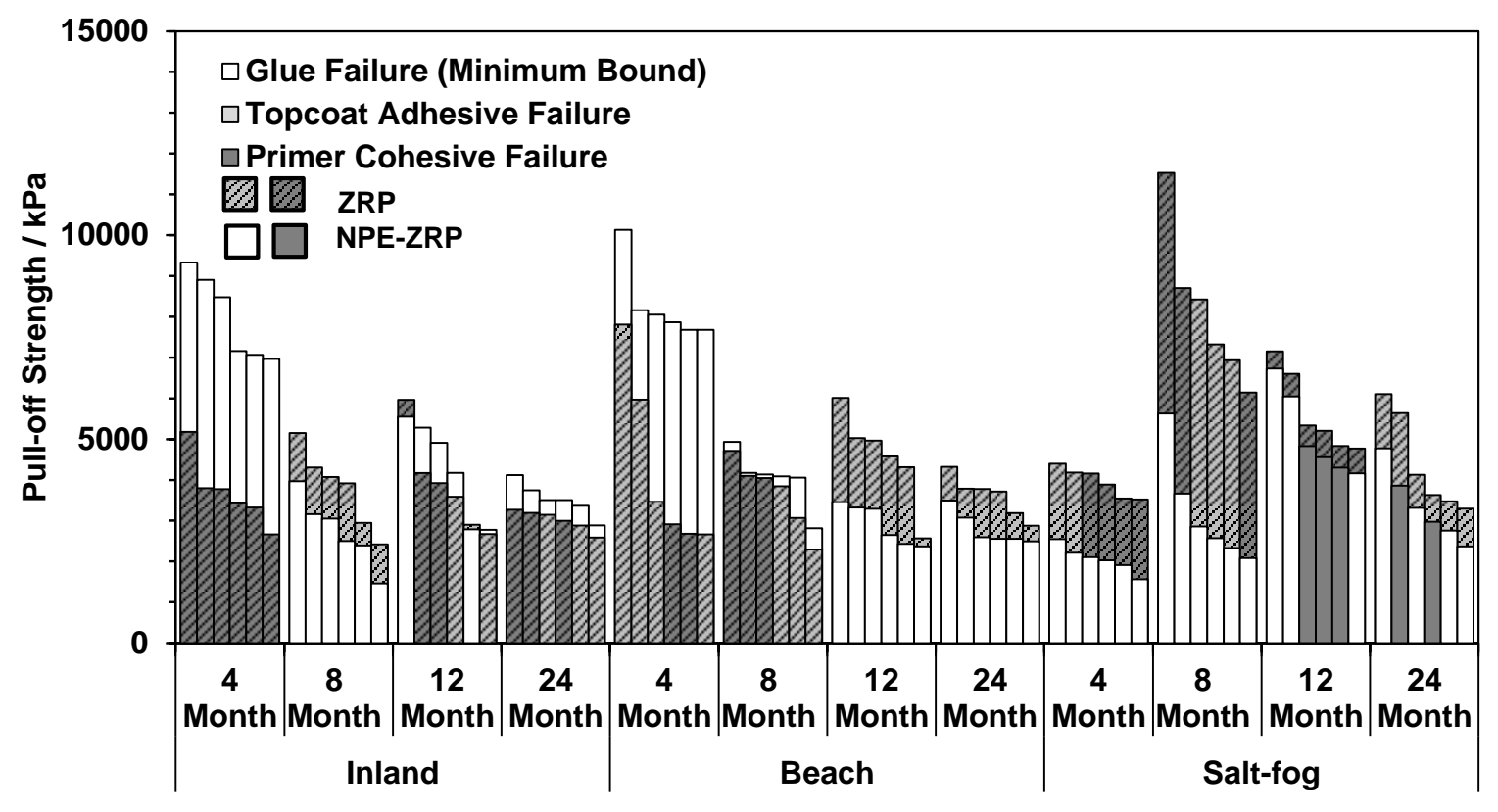

Figure 4.6. Pull-Off Strength of Outdoor \& Salt-fog Exposed Samples.

\subsubsection{Characterization of Zinc Consumption}

Coating degradation of samples from outdoor and salt-fog exposures was assessed by optical microscopy and photographic analysis. Image processing software was used to quantify the amount and modality of zinc consumption in consideration to the as-received zinc content by calculating the reduction of actual zinc area. 


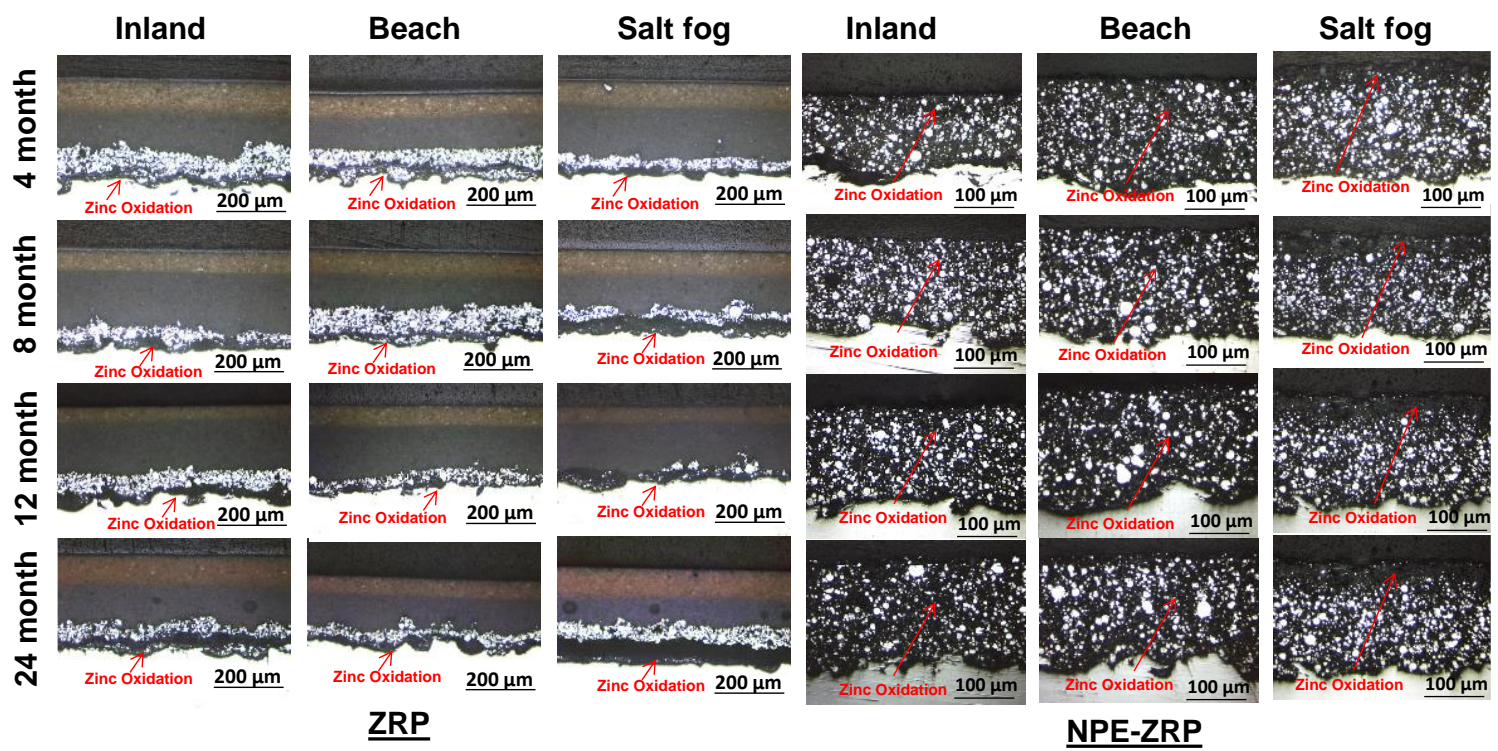

Figure 4.7. Degradation of the Outdoor and Salt-fog Exposed Unscribed Samples.

Figure 4.7 shows the micrographs of ZRP and NPE-ZRP unscribed samples at different times of exposure. The micrographs show the major characteristics of the ZRP and NPE-ZRP coatings. The ZRP coatings show the characteristic three-coat system with the zinc-rich primer, epoxy midcoat, and polyurethane topcoat. The NPE-ZRP coatings show a characteristic distribution of zinc pigments within the primer. After extended ambient atmospheric exposure, the ZRP samples showed some level of zinc consumption in the bulk primer, but it was apparent that there was a concentration of zinc pigment consumption at the primer/steel interface. The micrograph showed concentrated zinc consumption at the steel/coating interface of ZRP coating apparently creates a region susceptible to localized degradation which is consistent with the pull-off strength results of reduced mechanical adhesion. In contrast, the NPE-ZRP exhibited uniform consumption of the zinc pigments within the bulk primer layer. The micrographs did not indicate any significant levels of coating separation, attesting the enhanced mechanical 
properties of NPE-ZRP due to the presence of the nano-particles. Better continuity of the zinc pigments could also prevent concentrated zinc consumption as observed for ZRP. Predominant zinc consumption at the steel/primer interface of ZRP coating in contrast at the top surface of NPE-ZRP coating indicates a better barrier of the later apparently due to the improved barrier performance due to the pore filling by the nano-particles.

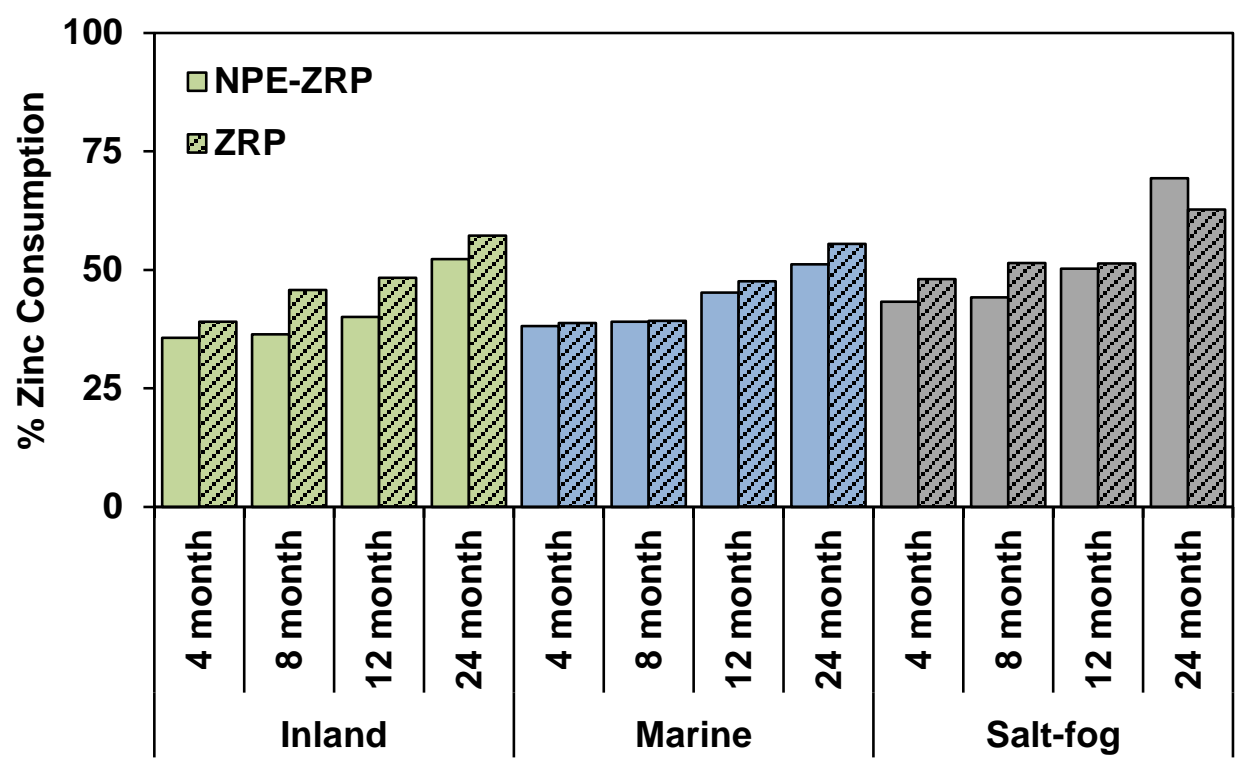

Figure 4.8. Zinc Consumption of Outdoor \& Salt-fog Exposed Unscribed Samples.

An image processing software was used to quantify the amount of zinc consumption in consideration of the as-received zinc content by calculating the reduction of the actual zinc area. Figure 4.8 shows the quantitative comparison of zinc consumption for both coatings along with the exposure duration for different environmental exposure. Evident zinc consumption within the primer matrix for both coatings indicates the porous nature of the epoxy matrix with the transport of electrolytes. The difference observed in modality and location of zinc consumption along with the primer layer between the two coatings. As expected, increment in the level of zinc consumption observed with exposure duration and the overall zinc consumption was higher for the ZRP in 
comparison to NPE-ZRP coating for all exposure conditions implies that greater degradation of the coating system when consumption continues along with the steel/primer layer. The comparative slower rate in zinc consumption after four months of exposure indicates the improved barrier effect of zinc oxidation products.

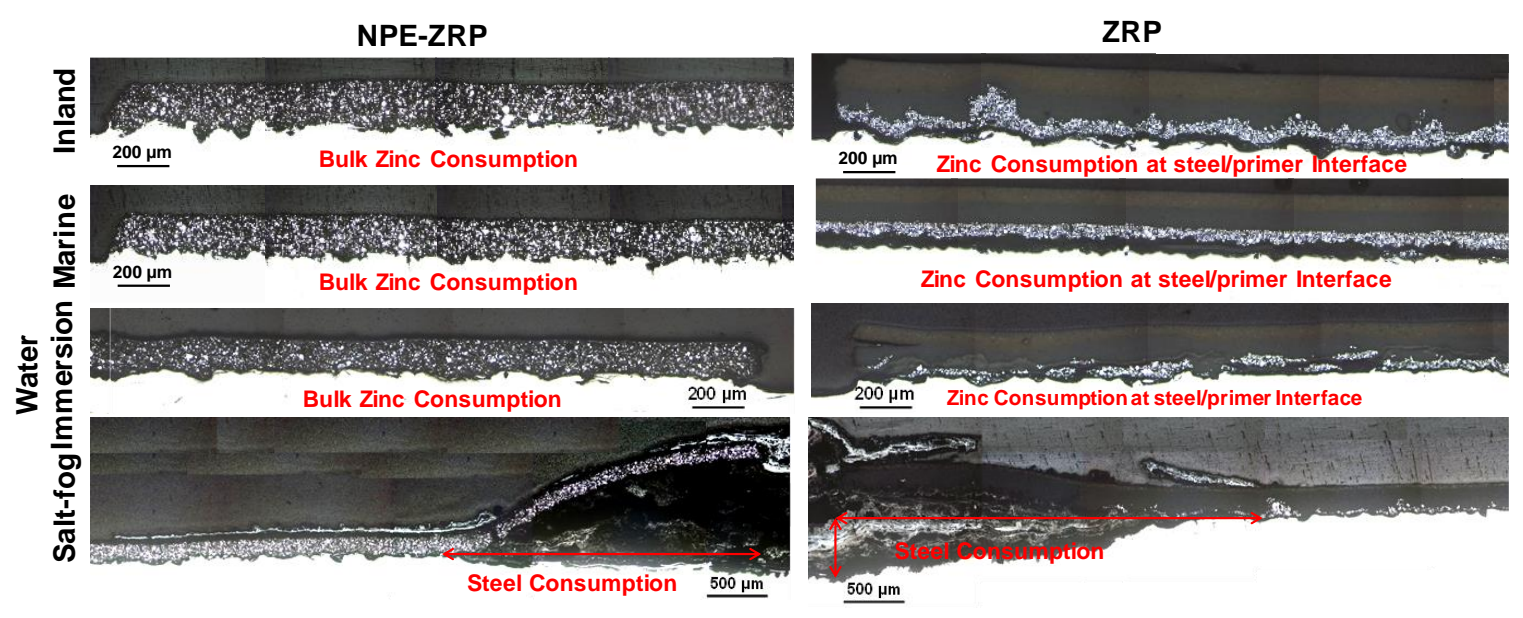

Figure 4.9. Degradation of the Exposed Scribed Samples (24 months Exposure).

Figure 4.9 shows the micrograph of scribed samples of both coatings from different environments after 24 months of exposure. Consistent with the unscribed samples zinc consumption observed for NPE-ZRP coating was from the bulk primer layer whereas for ZRP coating zinc consumption was concentrated along with the primer/steel interface for scribed samples. Initial zinc consumption started around the periphery of the coating defect for both coatings, dominant bulk consumption throughout the NPE-ZRP primer and major consumption along the coating/steel interface for the ZRP coating is clearly evident in the picture. Consumption throughout the primer layer for NPE-ZRP coating did not reveal significant localized zinc consumption at the defect site as like ZRP coating. This would further suggest some level of extended electrical continuity 
between the zinc pigments and with the steel substrate which is also evident by the higher mechanical bond from the pull-off strength result.

All the data is consistent with the general assertion of enhanced connectivity of zinc from the bulk for NPE-ZRP than ZRP as zinc consumption at the steel/coating interface can continue within a developing crevice type environment in the latter thus allowing greater degradation along with the coating interface. Contrastingly, the enhanced connectivity of the zinc pigments in the bulk coating for NPE-ZRP allowed for zinc to interact (at least for early exposure times) to provide some level of polarization with the activity of zinc in the bulk. Testing in salt-fog indicated that salts can be aggressive. The micrographs of the test samples after 24-month salt-fog exposure showed greater coating degradation, but the development of the coating degradation follow a similar sequence as described for outdoor exposure conditions. For ZRP, the heavy consumption of the zinc pigments occurred along an extended length along with the primer/steel interface. At coating defects, a similar mode was observed but the protection afforded by the zinc pigments could not accommodate the heavy steel corrosion accommodated by the greater moisture and salt availability. In contrast, NPE-ZRP with coating defects appeared to have better steel corrosion mitigation than ZRP where the enhanced mechanical properties and zinc pigment continuity allowed for a greater contribution of zinc pigments within the bulk primer. However, in the presence of coating defects, heavy steel corrosion developed due to the high level of moisture and salt present at the initial defect site that penetrated radially outwards.

\subsubsection{Water Immersion}

To identify the effect of continuous moisture presence on the corrosion mitigation 
mechanism of NPE-ZRP and ZRP coating, both the scribed and unscribed samples were exposed to $\mathrm{NaCl}$ aqueous solution for 60 days. Open circuit potential (OCP), corrosion current (LPR) and electrochemical impedance spectroscopy (EIS) data are represented in Figure 4.10-4.19, respectively.

\subsubsection{Electrochemical Analysis (OCP-LPR)}

Figure 4.10 and 4.11 shows the OCP \& LPR results of both NPE-ZRP \& ZRP coating during the 60 days of immersion. The initial OCP for unscribed NPE-ZRP samples was $\sim-0.4 \mathrm{~V}_{\mathrm{SCE}}$. The relatively noble potentials and the low corrosion current $(\sim 0.01 \mu \mathrm{A})$ likely indicate barrier protection provided by the epoxy primer. The shift of OCP in $\sim 1$ week to the more active condition of $\sim-0.6 \mathrm{~V}_{\mathrm{SCE}}$ was thought to be due to better ionic connectivity of the embedded zinc pigments to the outer test solution. It was posed that water penetration through the coating pore spaces allowed activation of zinc

pigments at the near-surface of the primer which was also observed by the white spot in samples from all exposure (Inland, Beach \& Salt-fog). ZRP samples showed similar behavior with an observed drop from initial noble potentials to more active potentials $0.6 \mathrm{~V}_{\mathrm{SCE}}$ and low corrosion current $(\sim 0.001 \mu \mathrm{A})$ throughout the test duration. The lower corrosion currents for ZRP than NPE-ZRP may be attributed by the additional midcoat and topcoat of the ZRP coating. 

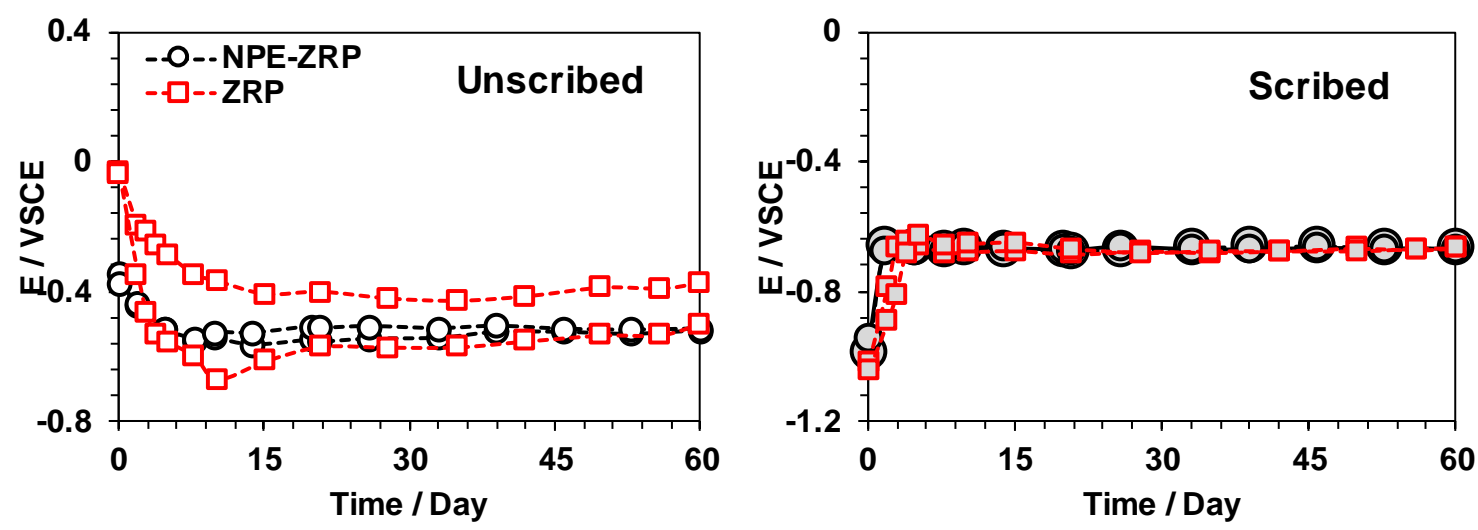

Figure 4.10. Open Circuit Potential during the Exposure.
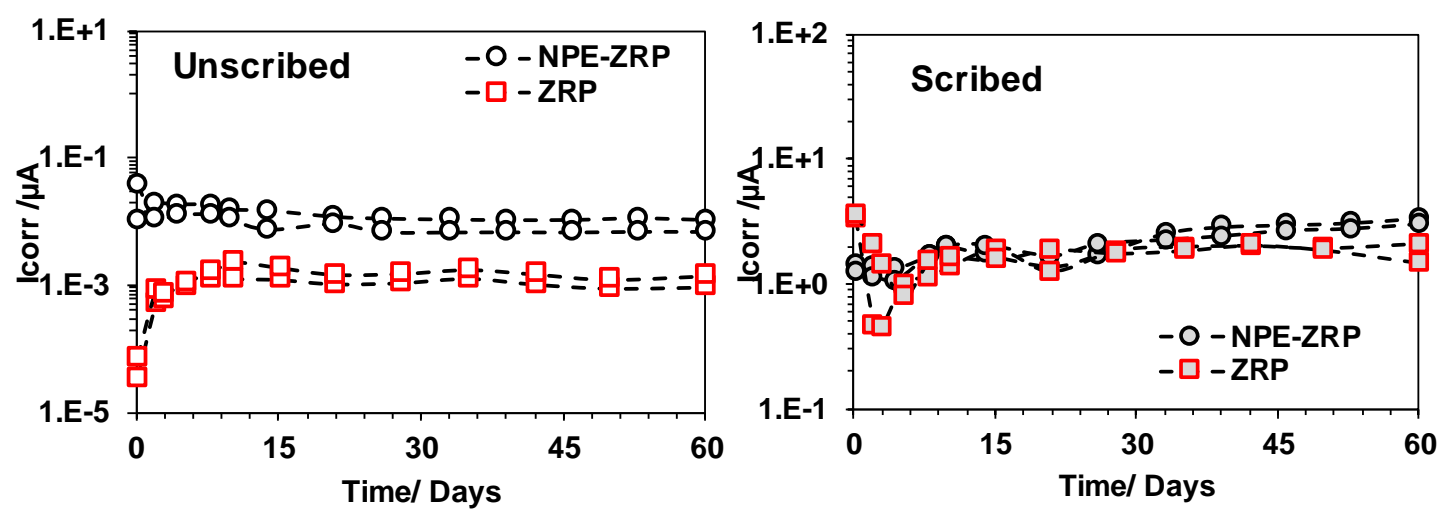

Figure 4.11. Corrosion Current over Exposure Duration.

For the scribed samples, similar behavior was observed for both NPE-ZRP and ZRP coatings. Active initial OCP condition of $\sim-1.0 \mathrm{~V}_{\mathrm{SCE}}$ was noted and was due to the early activation of exposed zinc pigments from the scribed defect region. Within two days of exposure, the OCP shifted to the more noble potential of $\sim-0.7 \mathrm{~V}_{\mathrm{SCE}}$ and maintained that value to the end of the test period. The exposed steel defects should ideally be cathodically protected. However, the early shift to more noble potentials may suggest less effective protection. Indeed, the steel corrosion was observed at the defect site after the first day of exposure. However, the terminal potential may provide some extent of beneficial galvanic coupling to the steel substrate. The corrosion current was 
relatively high throughout the test and had an apparent terminal value $>1 \mu \mathrm{A}$. This high current was measured after the apparent shift in the potential to passive-like conditions and in part accounts for the steel corrosion activity. Slightly higher corrosion current was measured for NPE-ZRP in comparison to ZRP coating apparently due to the enhanced conductivity for the incorporation of carbon nanotubes.

\subsubsection{Electrochemical Impedance Spectroscopy (EIS)}

\subsection{NPE-ZRP}

The EIS response (Nyquist, Bode and Phase Diagram) of the NPE-ZRP and ZRP samples were recorded for 60 days of immersion in $3.5 \% \mathrm{NaCl}$ solutions. Two replicate samples showed similar behavior, thus data for one representative sample is shown in Figure 4.12-4.13. Impedance responses were recorded at two different frequency ranges as $1 \mathrm{MHz}$ to $1 \mathrm{~Hz}$ and $100 \mathrm{kHz}$ to $1 \mathrm{mHz}$ to predict the corrosion-resistant properties of coatings. The high-frequency impedance range of $1 \mathrm{MHz}$ to $1 \mathrm{~Hz}$ used to determine the bulk coating degradation. A metal covered with an undamaged coating generally has very high impedance, but the absorption of electrolyte impedance gets reduced. The capacitance of a coated substrate changes as coating degrades with the absorption of water and EIS is used to measure that change. With the coating degradation, EIS resulted in more complex behavior as electrolyte penetration formed a new liquid/metal interface under the coating and corrosion is likely to occur at the interface. The low-frequency impedance range of $100 \mathrm{kHz}$ to $1 \mathrm{mHz}$ used to determine the coating degradation at the interface. 

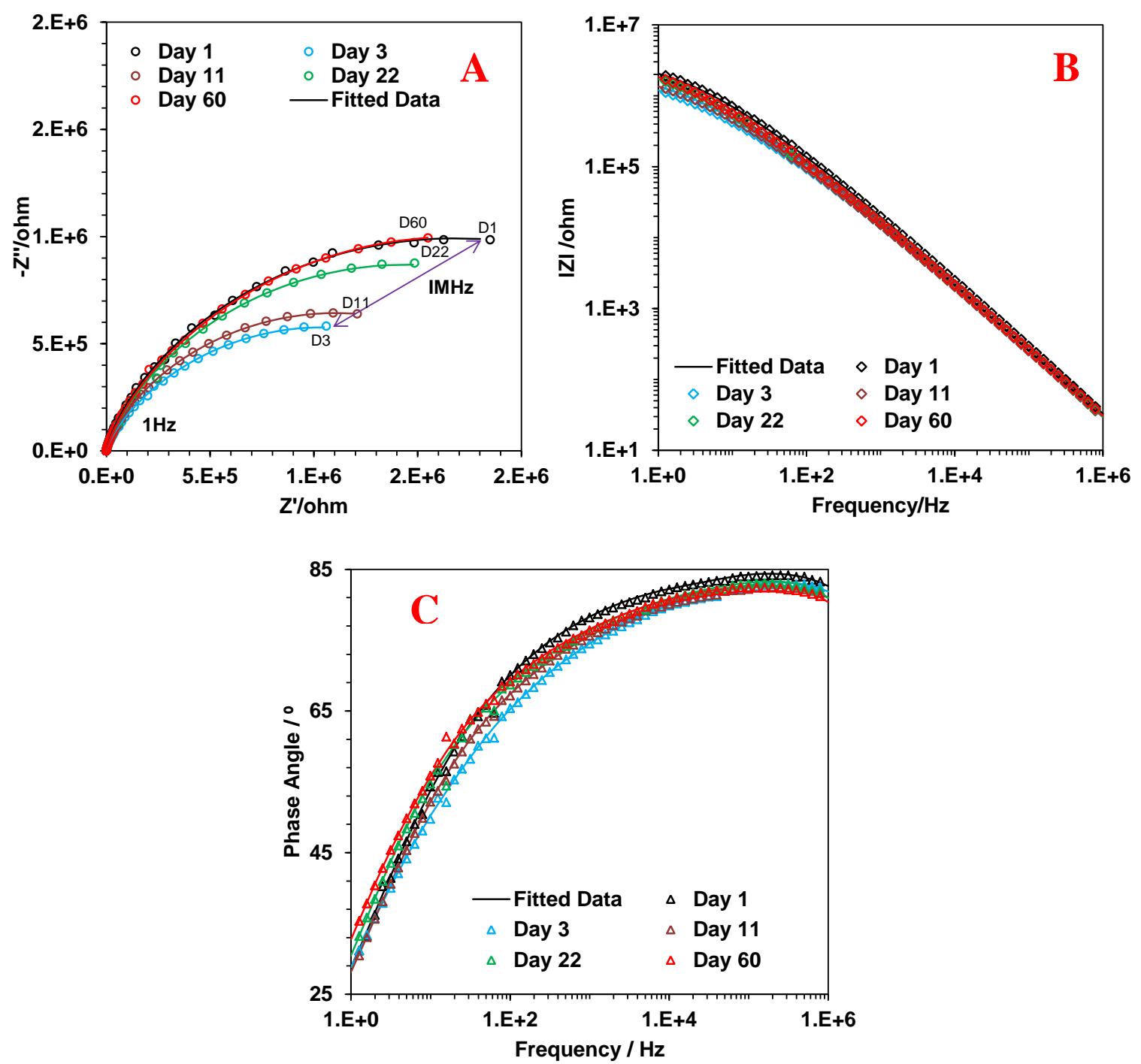

Figure 4.12. EIS response of NPE-ZRP Unscribed sample (1MHz to $1 \mathrm{~Hz})$.

A) Nyquist B) Bode C) Phase Diagram 

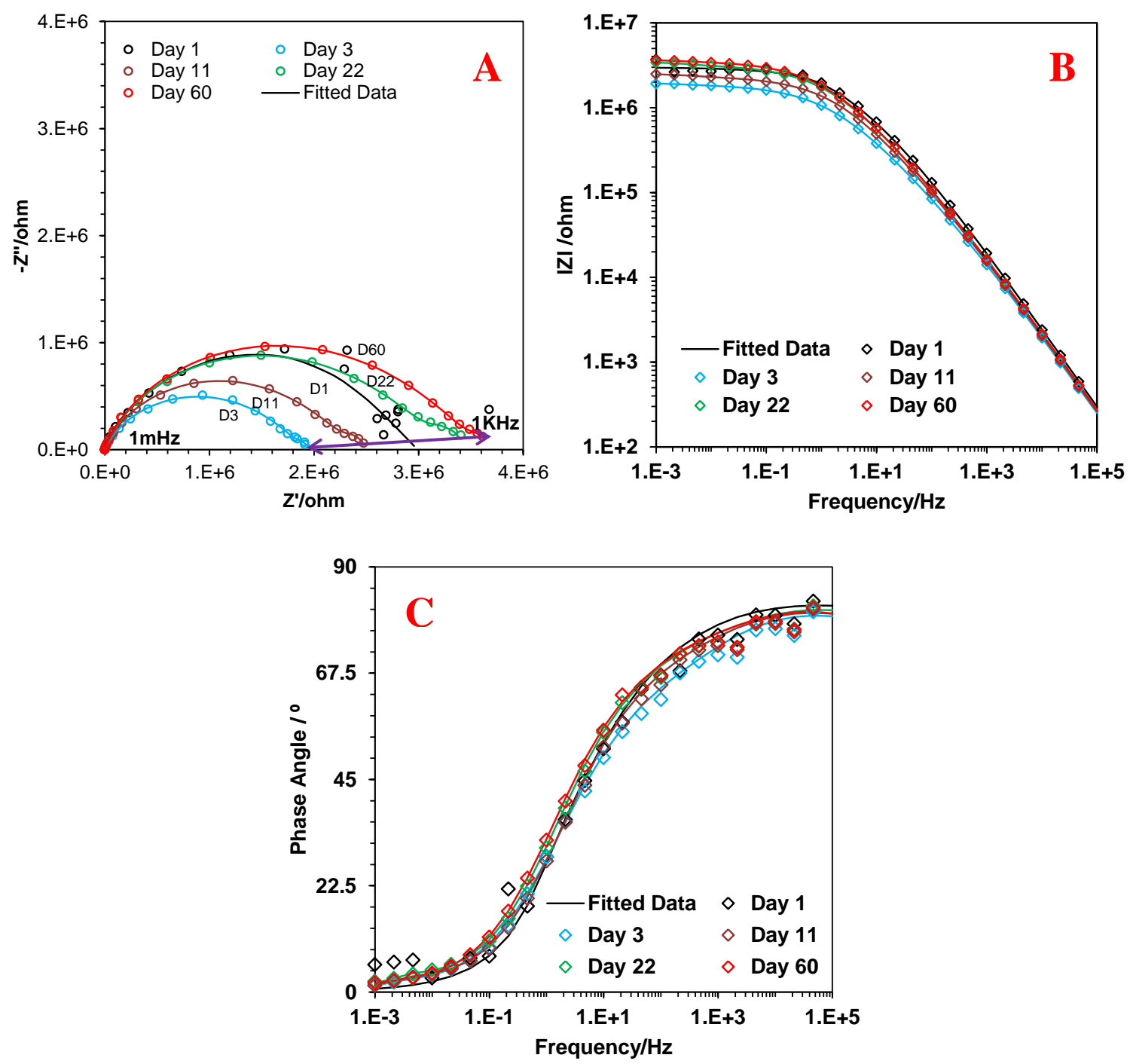

Figure 4.13. EIS response of NPE-ZRP Unscribed sample (100 kHz to $1 \mathrm{mHz})$. A) Nyquist B) Bode C) Phase Diagram

At high frequency response $(1 \mathrm{MHz}$ to $1 \mathrm{~Hz})$ Nyquist diagram showed one capacitive loop which is apparently a mixed impedance response from the barrier effect of the epoxy binder, contact impedances between the zinc particles due to the presence of zinc oxide that electrically insulates the zinc particles (Cubides and Castaneda, 2016 ) and electrochemical reactions taking place at the zinc particles surface (zinc oxidation and oxygen reduction reaction). The Nyquist diagram at first exhibits a reduction and 
then after 10 days of immersion, an increment observed in the capacitive loop. The impedance magnitude started to increase apparently due to the presence of zinc corrosion products that were formed during the cathodic protection mechanism. This behavior is in agreement with the change in the Nyquist loop at 11 days of exposure (at $100 \mathrm{kHz}$ to 1 $\mathrm{mHz}$ ), where another capacitive loop started to develop at the highest frequencies representing the formation of zinc corrosion product. This trend was observed during the remaining immersion time, which may indicate that a stable zinc oxide/zinc hydroxide layer was formed.

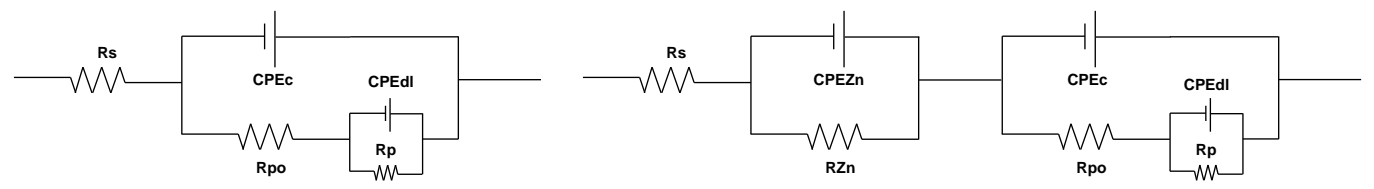

Stage 1

Stage 2

$$
Z=R_{S}+\frac{1}{Y_{o c}(j \omega)^{n c}+\frac{1}{R_{p o}+\frac{1}{Y_{n m}(j \omega)^{n m}+\frac{1}{R_{p}}}}}
$$

Stage 1

$$
Z=R_{S}+\frac{1}{Y_{o Z n}(j \omega)^{n Z n}+\frac{1}{R_{Z n}}}+\frac{1}{Y_{o c}(j \omega)^{n c}+\frac{1}{R_{p o}+\frac{1}{Y_{n m}(j \omega)^{n m}+\frac{1}{R_{p}}}}}
$$

\section{Stage 2}

\section{Figure 4.14. Equivalent Circuit for EIS Analysis of NPE-ZRP Unscribed Sample.}

Based on the impedance spectra, two different stages were identified during the immersion test. As an initial stage water uptake took place inside the epoxy binder, the complex presentation produces one semicircle. This can be associated with the mentioned 
mixed impedance response in which, dielectric properties of the binder, contact impedances, and activation of the zinc particles contribute to the global impedance response. At the beginning of the immersion time, the influence of the zinc particles in the impedance response was dominated by contact impedances, but the faradaic processes at the zinc particles became more relevant after several days of immersion. In the second stage, after three days of immersion, there was a noticeable change in the Nyquist representation due to the appearance of two capacitive loops.

Based on the OCP results, zinc activation was observed with time for the first ten days of immersion, and then, the potential increased. This behavior also is in agreement with the change in the Nyquist loop for 10 days of exposure, where another capacitive loop started to develop at the highest frequencies. This behavior represents the third stage in the corrosion degradation mechanism of this coating system. In addition to the capacitive loops representing the barrier properties of the coating, and the galvanic function provided by the zinc particles, the capacitive loop that started to develop at the highest frequency was related to the formation of a layer of zinc corrosion products primarily at the coating-electrolyte interface. This semicircle increased over time, which could be related to the continued growth of the protective layer and an extra barrier to subsequent permeation of oxygen and ionic species.

Figure 4.14 shows the electrical equivalent circuits used for impedance fittings of the NPE-ZRP unscribed sample. Two equivalent circuits were used to interpret the stages that were identified from the impedance response. For the water uptake stage Rs represents the electrolyte resistance between the reference electrode and the coated samples, and CPEc and Rpo describe the dielectric properties of the epoxy binder and 
also the contribution of contact impedances between the zinc particles. $\mathrm{CPE}_{\mathrm{dl}}$ and $\mathrm{Rp}$ showed in stage II represent the double-layer capacitance and the charge transfer resistance at the interface between the zinc particles and the electrolyte. For all equivalent circuits, constant phase elements (CPE) were used instead of capacitances due to surface heterogeneities, deviation from capacitive behavior, and dispersion effects. The impedance for a CPE is defined as:

$$
Z_{C P E}=\frac{1}{Y_{O}(j \omega)^{n}}
$$

where Yo and $\mathrm{n}$ represent the admittance and empirical exponent of the constant phase element, $\mathrm{j}$ is the imaginary number, and $\omega$ is the angular frequency.

Figure 4.15 shows the evolution of the different equivalent circuit elements for NPE-ZRP unscribed samples with time. The impedance of higher frequencies reflects the physical properties of the coating, including the formation of corrosion products, whereas the electrochemical processes dominate the responses at lower frequencies. For unscribed samples during the 60 days immersion time, the high-frequency range ( $1 \mathrm{MHz}$ to $1 \mathrm{~Hz})$ is used to determine the level of coating degradation. Figure A shows the solution resistance and the apparent constant value indicated no bulk degradation of the coating system that can affect the electrolyte. Figure B shows the coating pore resistance, which rapidly decreased during the first few days of immersion because of the diffusion of electrolyte through a large number of pores inside the epoxy coating matrix, causing the activation of zinc particles and decreasing the contact impedances between them. After 10 days of immersion, there was an increment of the coating resistance due to the formation of zinc corrosion. Progressive increment or apparent constant capacitance during the remaining 
exposure time apparently as a result of the formation of zinc corrosion products on the coating surface that prevented additional diffusion of the electrolyte to the epoxy matrix. Figure $\mathrm{C}$ shows the water uptake by the coating layer during the immersion time. During the first two weeks, there was a gradual increment of the water content due to uptake. With the progression of the immersion time, the water content remained almost constant because of the saturation of the epoxy matrix. Figure D shows the effective coating capacitance of the NPE-ZRP coating system. Coating capacitance was calculated from the CPE using Hsu and Mansfeld method. Capacitance increased during the first few days of immersion as a result of the diffusion of electrolyte through the coating system. With the progression of the immersion time, the capacitance values of the coating system remained almost constant because of the saturation of the epoxy with the electrolyte.
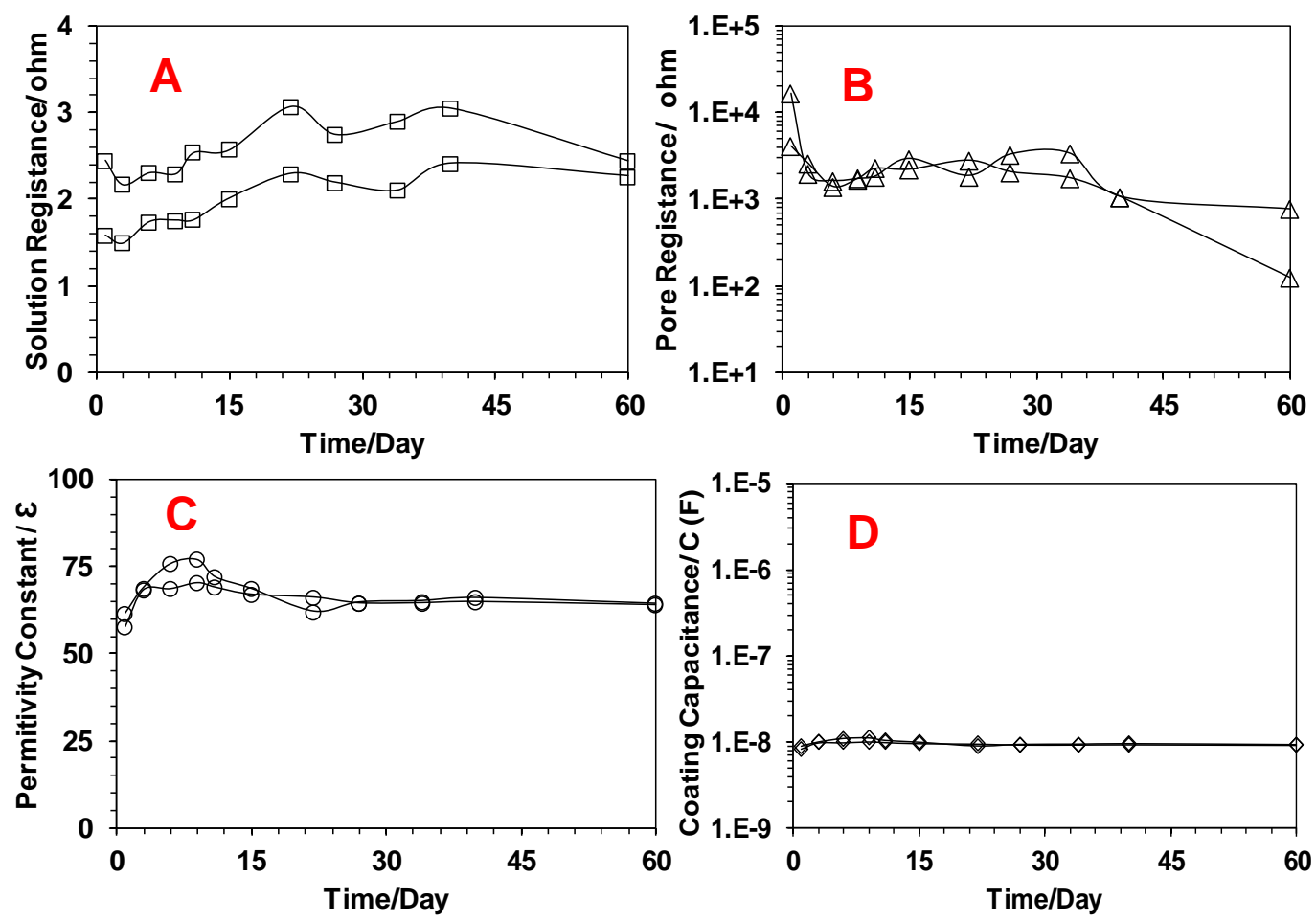

Figure 4.15. Equivalent Circuit Elements for NPE-ZRP Unscribed Samples. A) Solution Resistance B) Pore Resistance C) Water Content D) Coating Capacitance 
The EIS response of the NPE-ZRP scribed samples at $100 \mathrm{kHz}$ to $1 \mathrm{mHz}$ frequency range during the 60 days immersion in $3.5 \% \mathrm{NaCl}$ solutions are shown in Figure 4.16 (two replicate samples showed similar behavior, thus data for one representative sample is shown).
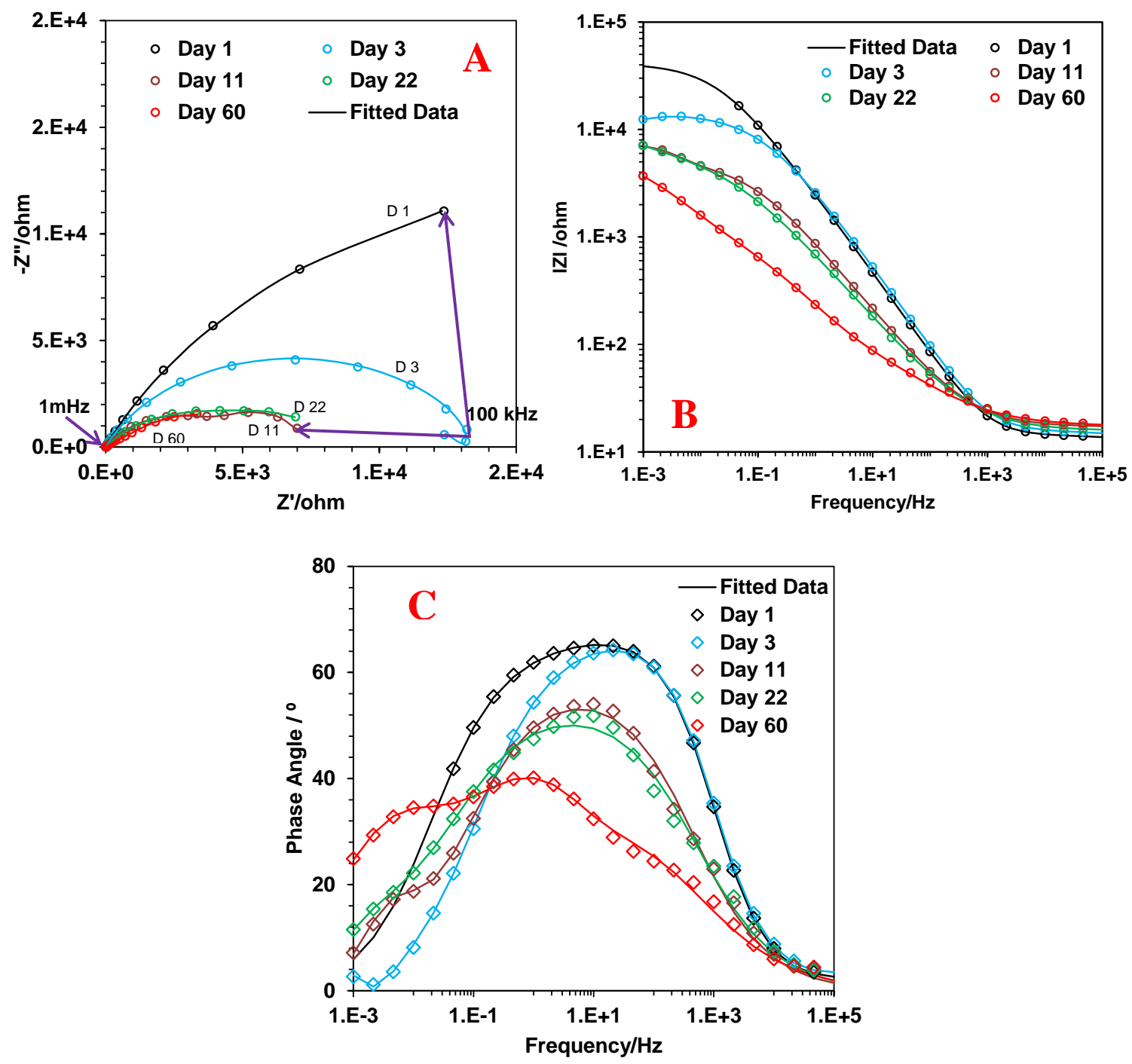

Figure 4.16. EIS response of NPE-ZRP Scribed sample (100 kHz to $1 \mathrm{mHz})$. A) Nyquist B) Bode C) Phase Diagram

Scribed samples consist of a $25.4 \mathrm{~mm}$ long and $0.5 \mathrm{~mm}$ wide introduced coating defect exposing the steel substrate. Impedance spectra of NPE-ZRP showed mostly 
capacitive behavior during the entire immersion test. Generally, for the damaged NPEZRP coating, the zinc particles around the defect sacrificially dissolved and provide cathodic protection to the steel substrate which was also observed in the OCP potential. But the cathodic potential was active only during the first day of exposure and steel corrosion product was observed on the second day of exposure. The gradual decrease in

the impedance with exposure indicated this phenomenon that the sacrificial dissolution of zinc pigments could no longer inhibit the dissolution of the steel substrate.

\subsection{ZRP}

The EIS response of the ZRP unscribed samples for 60 days immersion in $3.5 \%$ $\mathrm{NaCl}$ solutions are shown in Figure 4.17-4.18 (two replicate samples showed similar behavior, thus data for one representative sample is shown). Impedance responses were recorded at two different frequency ranges as $1 \mathrm{MHz}$ to $1 \mathrm{~Hz}$ and $100 \mathrm{kHz}$ to $1 \mathrm{mHz}$ to predict the corrosion-resistant properties of paints.

At high frequency response $(1 \mathrm{MHz}$ to $100 \mathrm{~Hz})$ Nyquist diagram showed one capacitive loop (Figure 4.17) which is apparently a mixed impedance response from the barrier effect of the epoxy binder, contact impedances between the zinc particles due to the presence of zinc oxide, and electrochemical reactions taking place at the zinc particles surface (zinc oxidation and oxygen reduction reaction). With the time of immersion Nyquist diagram shows a depressing trend in the capacitive loop. The impedance magnitude started to decrease due to the continuous degradation of the epoxy matrix and another capacitive loop started to develop at the low-frequency range. This trend was observed during the remaining immersion time, which may indicate the continuous dissolution of zinc pigments following the least resistive path. 
Bode representation of ZRP showed mostly capacitive behavior during the entire immersion test. Resistive behavior also exhibits in the low-frequency impedance response (100 kHz to $1 \mathrm{mHz})$.

Indeed, water uptake was observed on the first day of immersion and was continuous during the entire exposure period. This behavior can be associated with the water uptake through the capillary transport of electrolytes due to the porosity of the coating, which enables galvanic influence by the zinc particles and continuous degradation of the coating system. However, during the entire test period, ZRP showed high impedance values of $10 \mathrm{E}^{7-8} \mathrm{ohm}$ which is close to the reported resistance of barrier performance of the organic coating. The barrier performance of ZRP is apparently attributed to the three-layer of the polymeric epoxy matrix. Formation of another capacitive loop apparently due to the presence of zinc corrosion products that were formed due to electrolyte penetration. This behavior is in agreement with the change in the Nyquist loop (Figure 4.18) at 11 days of exposure (at $100 \mathrm{kHz}$ to $1 \mathrm{mHz}$ ). This trend was observed during the remaining immersion time, which may indicate that a stable zinc oxide/zinc hydroxide layer was formed. 

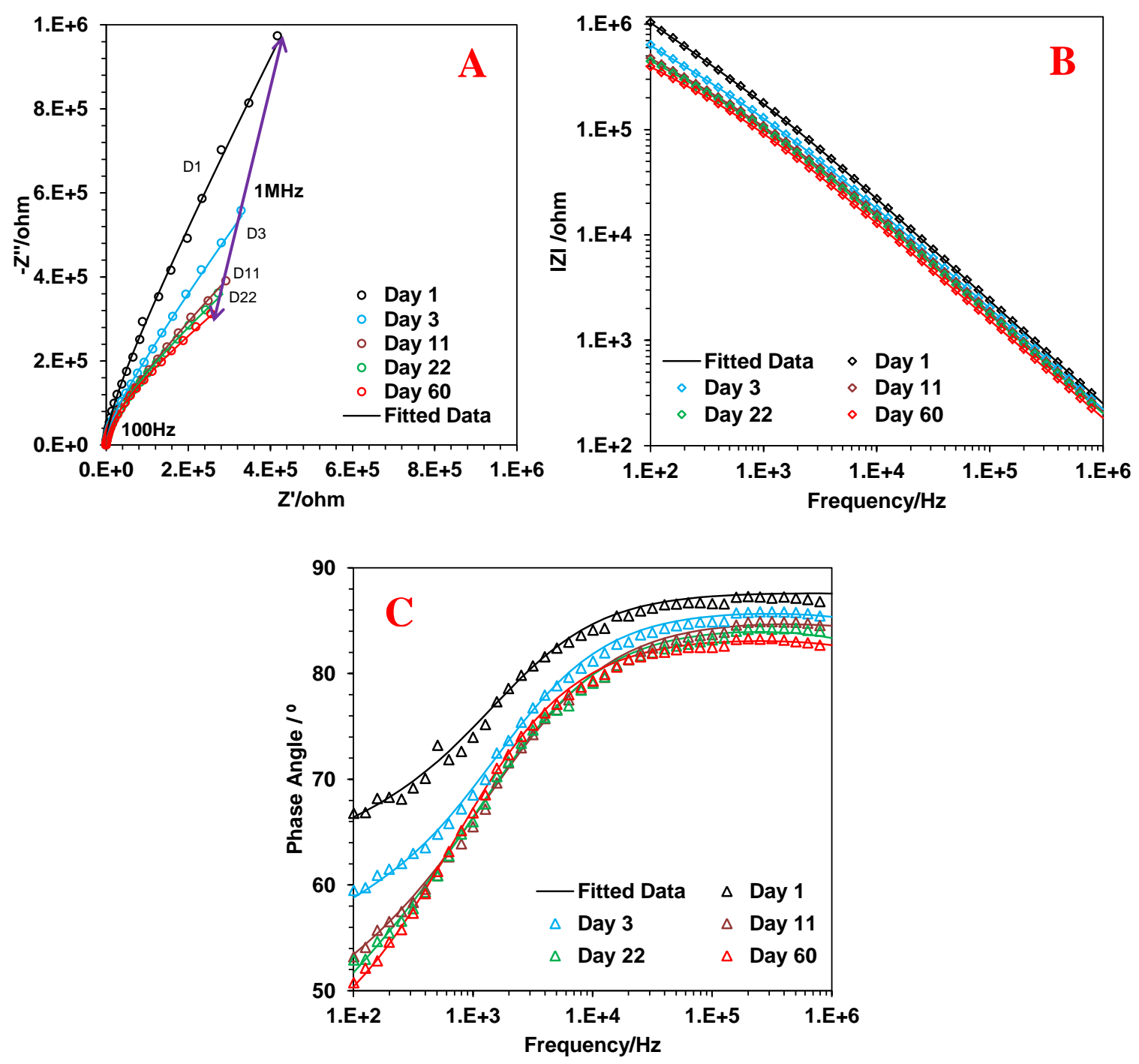

Figure 4.17. EIS response of ZRP Unscribed sample (1MHz to $1 \mathrm{~Hz})$.

A) Nyquist B) Bode C) Phase Diagram 

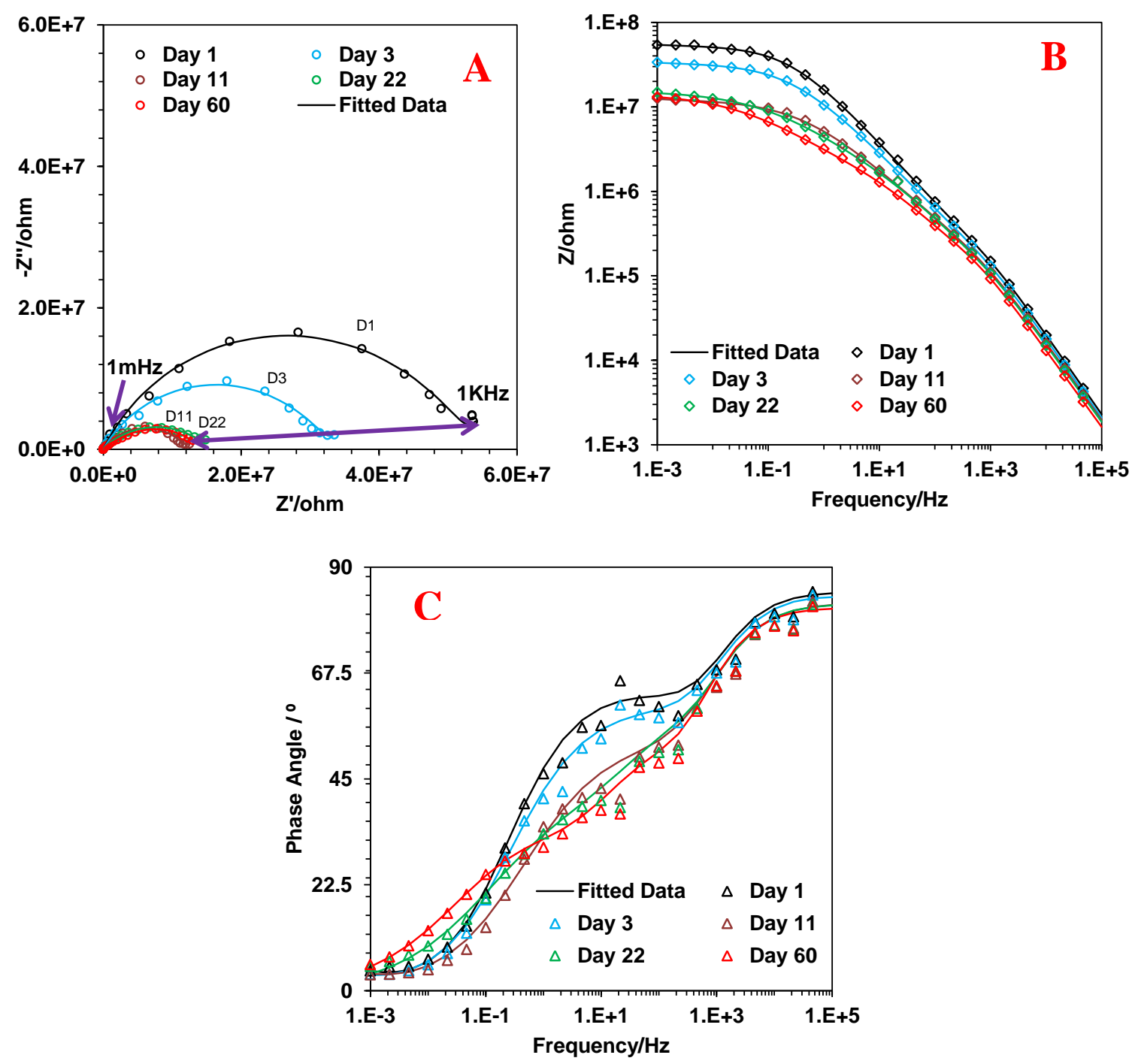

Figure 4.18. EIS response of ZRP Unscribed sample (100 $\mathrm{kHz}$ to $1 \mathrm{mHz})$. A) Nyquist B) Bode C) Phase Diagram 

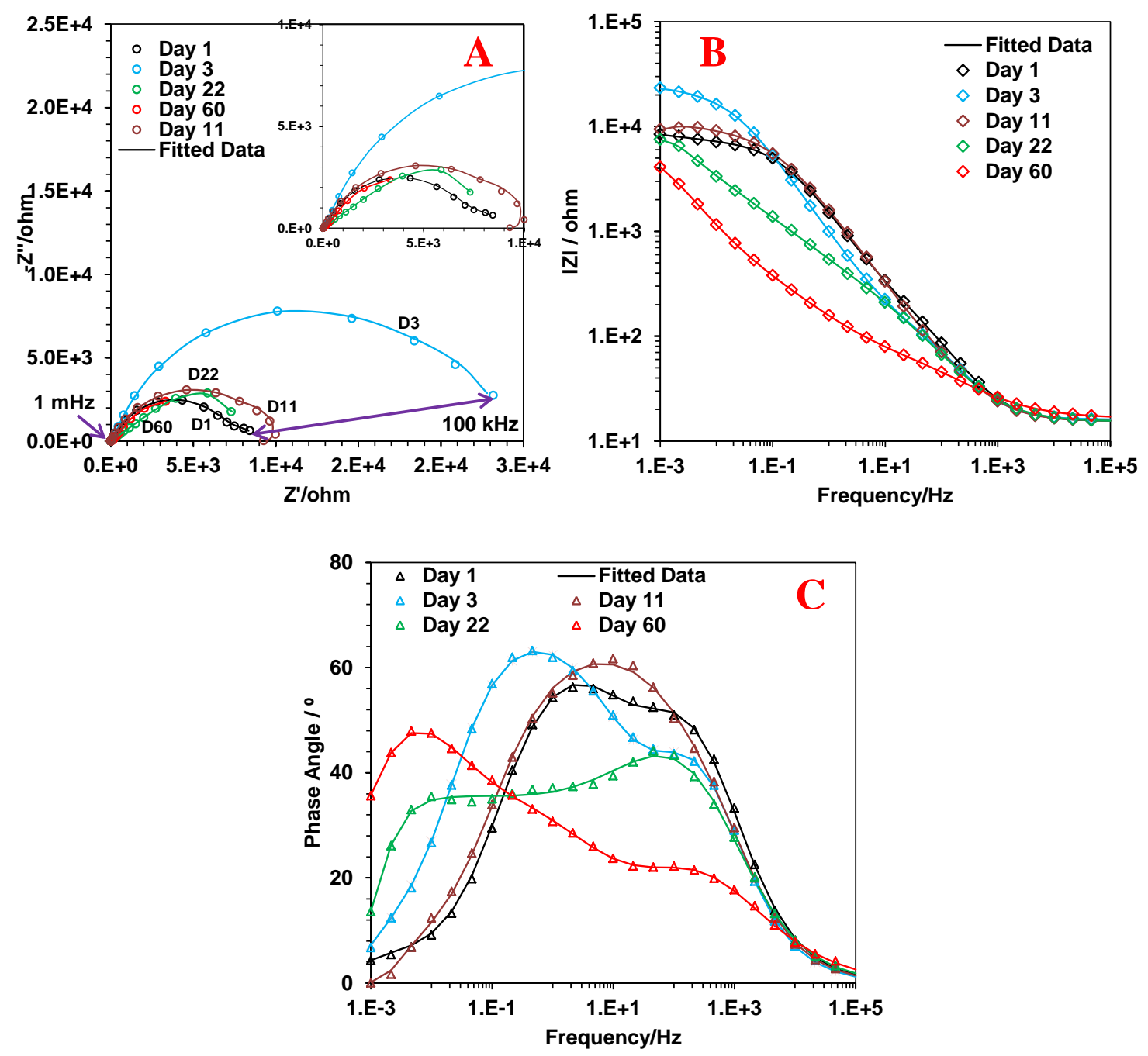

Figure 4.19. EIS response of ZRP Scribed sample (100 $\mathrm{kHz}$ to $1 \mathrm{mHz})$.

A) Nyquist B) Bode C) Phase Diagram

The EIS response of the ZRP scribed samples at $100 \mathrm{kHz}$ to $1 \mathrm{mHz}$ frequency range during the 60 days immersion in $3.5 \% \mathrm{NaCl}$ solutions are shown in Figure 4.19 (data for one representative sample is shown). Scribed samples consist of a $25.4 \mathrm{~mm}$ long and $0.5 \mathrm{~mm}$ wide introduced coating defect exposing the steel substrate. Impedance spectra of ZRP showed mostly capacitive behavior during the entire immersion test. There was an increment in the impedance magnitude at the lowest frequency $(1 \mathrm{mHz})$ apparently due to the formation of resistance from the cathodic dissolution of zinc 
pigments. Generally, for the damaged ZRP coating, the zinc particles around the defect sacrificially dissolved and provide cathodic protection to the steel substrate which was also observed in the OCP potential. But the cathodic potential was active only during the first day of exposure and indeed steel corrosion product was observed on the second day of exposure.

\subsubsection{Visual Observation}

Visual observation of the unscribed and scribed samples from the water immersion test, for both NPE-ZRP and ZRP coating after 60 days of immersion in 3.5\% $\mathrm{NaCl}$ solution is shown in Figure 4.20. The discoloration was observed for unscribed samples of both coatings and was more predominant for NPE-ZRP samples as white rust (oxidation of exposed zinc pigments from the top surface) deposited over the area which was exposed to the electrolyte. Degradation of the ZRP coating occurred due to zinc oxidation and degradation of polymer layers because of the hydrolysis of polymer chain due to diffusion of electrolyte. Localized rust (iron oxidation) formation was observed in scribe defect for both coatings. OCP results also consistent with the formation of iron oxidation products as cathodic protection was active only for the first two days after immersion. However, on the NPE-ZRP samples, white staining was more predominant that may be related to zinc corrosion product (white rust) from the exposed top layer, formed throughout the portion of the coupon surface exposed to continuous solution immersion. 


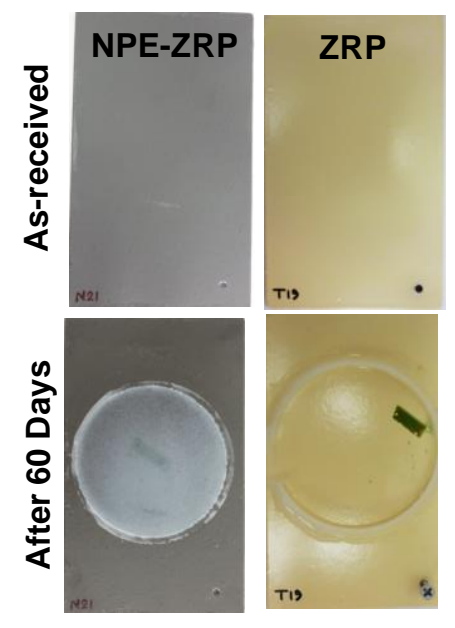

Unscribed

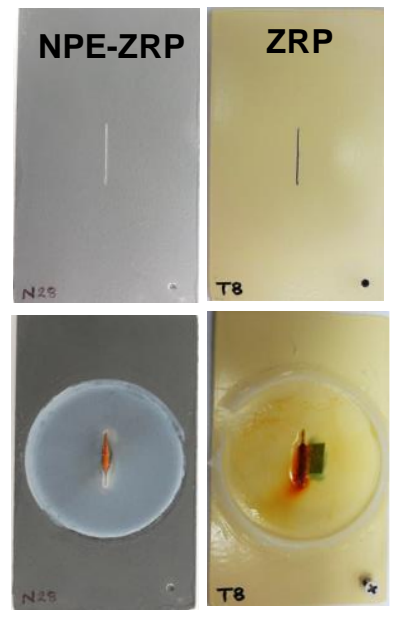

Scribed

Figure 4.20. Condition of the Water Immersed Samples.

\subsubsection{Coating Thickness}

Change in coating thickness due to 60 days of immersion in the salt solution is presented in Figure 4.21. NPE-ZRP samples showed an increase in coating thickness after exposure consistent with the visual observation of white staining which is due to the accumulation of oxidation product of exposed zinc pigments. A decrease in coating thickness after exposure for ZRP samples apparently due to the degradation of the polymer due to hydrolysis from the constant immersion in the salt solution. The change in thickness due to continuous immersion in saltwater is consistent with the behavior in outdoor and salt-fog exposed samples. 


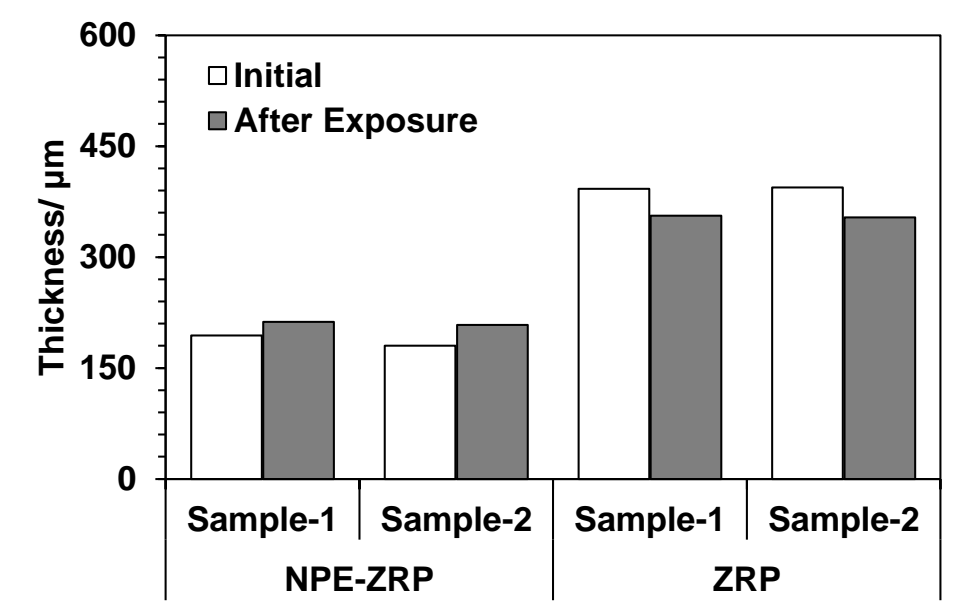

Figure 4.21. Change in Coating Thickness after Water Immersion.

\subsubsection{Pull-off Strength Test}

The results of the pull-off strength test and failure modality of both NPE-ZRP and ZRP coatings after exposure are shown in Figure 4.22. Testing of the water-immersed NPEZRP sample resulted in glue failure at values greater than $\sim 7,000 \mathrm{kPa}$ represented here as minimum bound. However, the pull-off strengths for NPE-ZRP were higher than for ZRP samples, indicating enhanced bond strength for the former. For ZRP coatings after water immersion, testing resulted in low pull-off strength. The modality of coating failure was topcoat adhesive or primer cohesive failure. The results for ZRP were similar to values measured from the as-received samples and 60 days duration of laboratory testing in immersed salt solution did not show strong supporting evidence of the effect of prolonged salt solution exposure. Even though testing did not reveal any discernible effects by continuous moisture exposure, there can possibly create a region susceptible to localized degradation which is related to some extent of bond strength degradation compared to the as-received condition. 

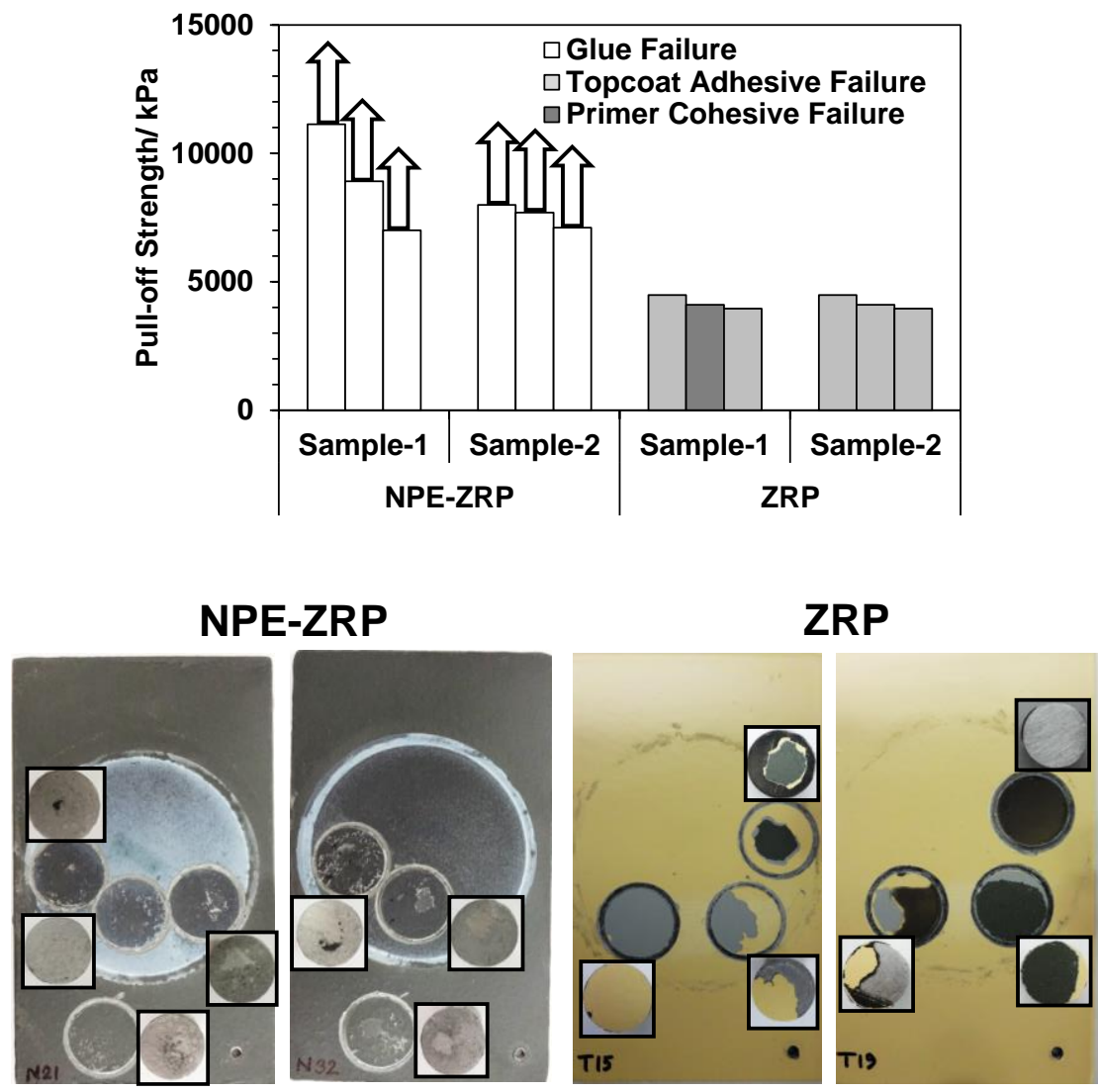

Figure 4.22. Pull-off Strength and Failure Modality of Samples after Immersion. (Arrow indicates apparent higher value)

\subsubsection{Characterization of Zinc Consumption}
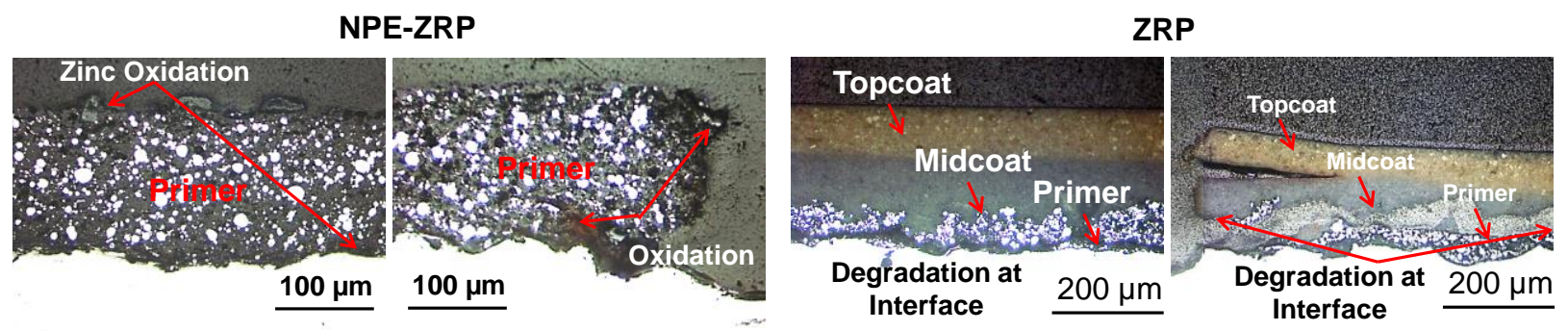

Figure 4.23. Degradation of the Water Immersed Samples.

Cross-sectional micrograph for both coatings after the solution immersion exposure is shown in Figure 4.23. As seen in Figure 4.23, for unscribed samples zinc 
corrosion occurred at the top exposed layer of NPE-ZRP coating which is consistent with the increment of thickness after exposure as the volume of oxidation product is greater than the native zinc pigments. With the exposure duration, zinc oxidation occurred through the bulk primer layer of the NPE-ZRP coating. Whereas for the ZRP samples zinc consumption was concentrated at the steel/primer interface. For the scribed samples zinc oxidation occurred around the periphery of the scribe region for both coatings. After the initial consumption around the defect site, the zinc consumption for NPE-ZRP coating was from the bulk primer layer and for ZRP coating it was along with the steel/primer interface. Uniform oxidation of zinc pigments from within the bulk primer layer that occurred for the NPE-ZRP coating during water immersion did not result in significant degradation of coating adhesion. However, the concentrated zinc consumption at the steel/coating interface of ZRP coating apparently creates a region susceptible to localized degradation which is consistent with the pull-off strength results.

\subsubsection{Electrochemical Polarization}

\subsubsection{Visual Observation}

The visual appearance of NPE-ZRP and ZRP coated samples after 30 days of exposure to OCP, cathodic and anodic polarization regime in $3.5 \% \mathrm{NaCl}$ solution is represented in Figure 4.24. In OCP and $-600 \mathrm{mV}$ SCE polarized conditions, steel corrosion product was identified on the steel surface within the coating defect and whereas significant steel corrosion was observed for $-300 \mathrm{mV}$ SCE and $+100 \mathrm{mV}_{\text {SCE }}$ polarization. As expected, cathodically polarized samples did not show steel corrosion deterioration at the exposed steel surface after a month of polarization. White rust resulted from the oxidation of exposed zinc pigments from the top surface layer was observed for all NPE- 
ZRP coating samples except for $-300 \mathrm{mV}_{\text {SCE }}$ and $+100 \mathrm{mV}_{\text {SCE }}$ polarization level where steel corrosion was predominant. Only ZRP samples showed coating blistering near the defect site for the $-1500 \mathrm{mV}_{\mathrm{SCE}}$ and the $-1100 \mathrm{mV} \mathrm{VSE}_{\mathrm{SC}}$ cathodic polarization cases due to the large impressed cathodic currents as well as imperfection within the coating.

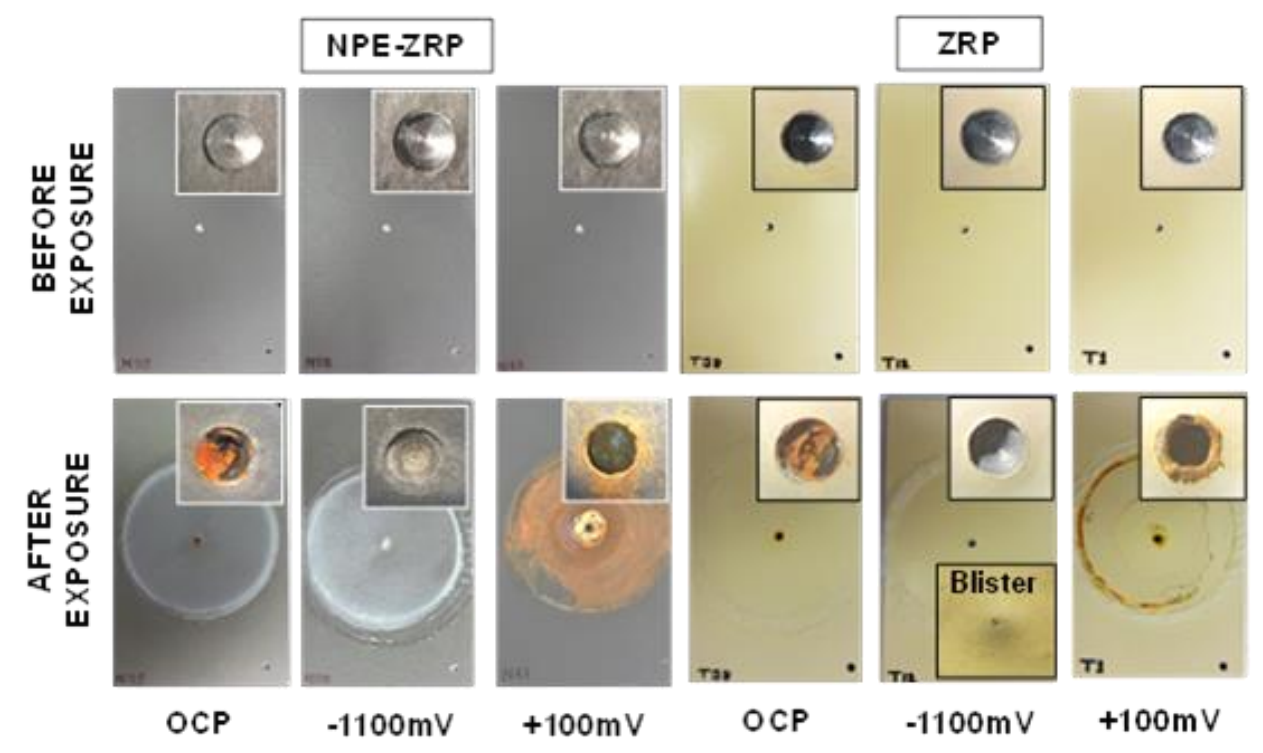

Figure 4.24. Condition of the Representative Samples before and after Exposure.

\subsubsection{Electrochemical Analysis (OCP-LPR)}

The measured open-circuit potentials (OCP) and corrosion currents with time are shown in Figure 4.25 and Figure 4.26, respectively. The efficacy of cathodic protection system for zinc-rich coatings is primarily perceived by the evolution of OCP of the coupled system. The electrochemical processes in zinc-rich coating systems are usually the oxidation of exposed zinc pigments and the reduction of dissolved oxygen. Metallic zinc showed the active potential of $\sim 1.0 \mathrm{~V}_{\mathrm{SCE}}$ when exposed and provide galvanic protection. Active cathodic protection duration is defined by the period where OCP remains lower than $-0.86 \mathrm{~V}_{\mathrm{SCE}}$, of a maximum $\mathrm{Fe}^{2+}$ concentration in $10^{-6} \mathrm{M}$ (Hammouda et al., 2011). This corresponds to the reduction of cathodic efficacy with the electroactive 
zinc area by the oxidation reaction and formation of zinc corrosion products (Abreu et. al., 1999). For both NPE-ZRP and ZRP samples, an active initial OCP of $\sim-1.0 \mathrm{~V}_{\text {SCE }}$ was observed due to the early activation of the exposed zinc pigments in the defect region. Within one day of exposure, the OCP shifted to more noble potentials $>-0.750 \mathrm{~V}_{\text {SCE }}$ and stayed in this passive-like mixed-potential condition to the end of the test period. NPEZRP samples showed significant noise fluctuations during its transition to more noble potential and reached a stable condition very quickly (as fast as 3 hours after immersion). The exposed steel at the defect site for both NPE-ZRP and ZRP should ideally have beneficial galvanic coupling, but the early shift to more noble potentials may suggest less effective protection. Indeed, some level of steel corrosion was observed at the defect sites for all samples (both NPE-ZRP and ZRP) on the second day of exposure. This apparently implies that coating defect allows the electrolyte to activate the exposed zinc pigments and during the first day of exposure they were protecting the uncovered steel substrate. With zinc dissolution active zinc to steel area got reduced due to the formation of zinc corrosion products around the zinc pigments, cathodic efficacy became less effective due to a decrease in the electrical contact between zinc particles as well as with the steel substrate and the steel substrate started corroding slowly. 


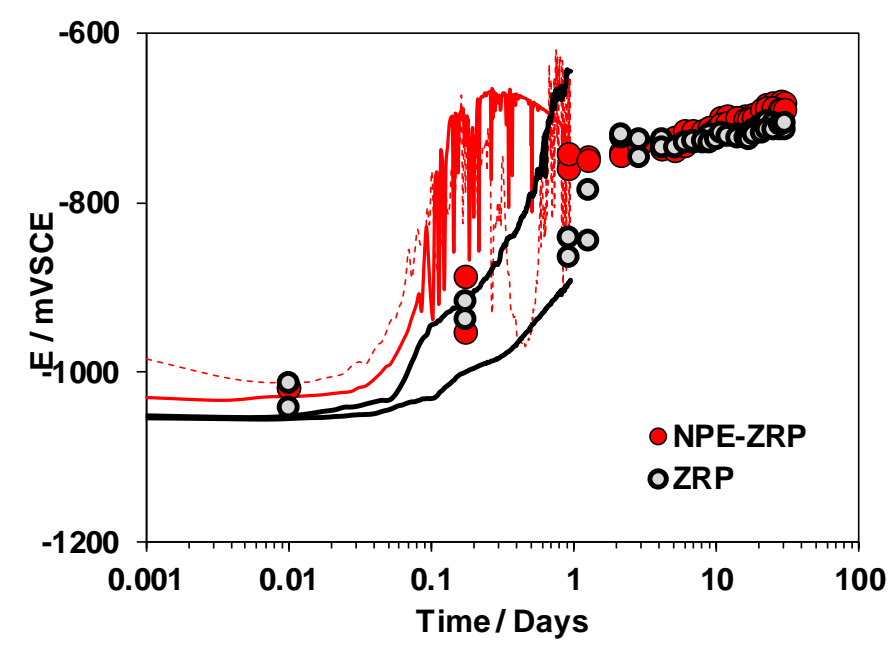

Figure 4.25. OCP\& LPR evolution of NPE-ZRP and ZRP with Time.

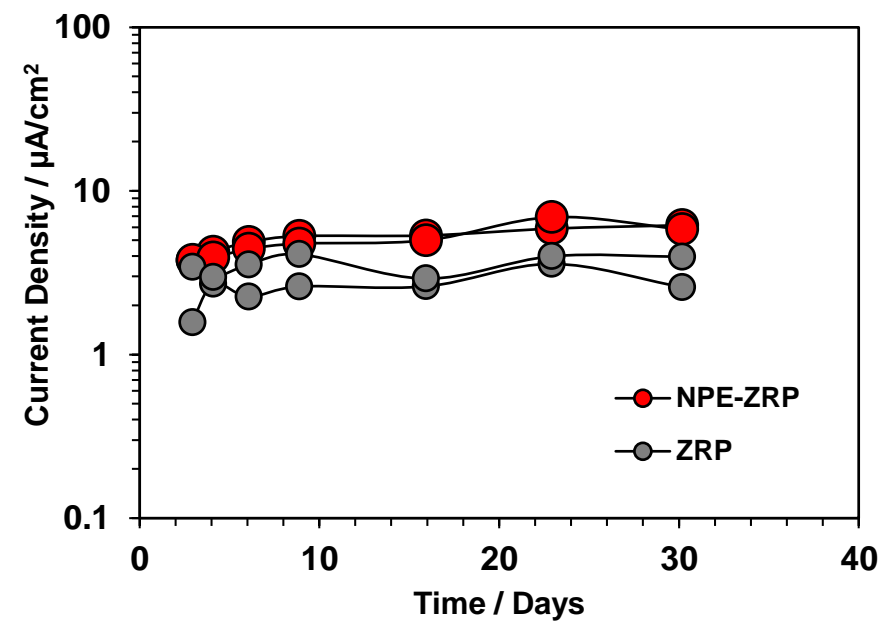

Figure 4.26. OCP\& LPR evolution of NPE-ZRP and ZRP with Time.

Due to the early transient behavior of the active zinc pigments described above, corrosion currents could not be measured. Stable behavior to accommodate linear polarization resistance measurements were not observed until day 2 where passive-like conditions for all samples already developed. At that time and until the end of testing at day 30 , the nominal current density for NPE-ZRP and ZRP were $\sim 2 \mu \mathrm{A} / \mathrm{cm}^{2}$ although current densities appeared slightly higher for NPE-ZRP. A comparative higher corrosion rate can be attributed from the local galvanic cells between the zinc pigments through the 
better connectivity by the carbon nano-particles and also between nano-particles and steel substrate. This behavior was observed by Fukuda, et al. 2011, where CNTs apparently increased the corrosion rates of magnesium in CNT-Mg composites in a $3.5 \%$ wt $\mathrm{NaCl}$ solution as a result of galvanic corrosion between the magnesium and the CNTs. Arronche, et al. 2013, also observed that the addition of CNTs into a carbon fiberreinforced polymer coating slightly increased the corrosion rate of the steel substrate during immersion in $2 \% \mathrm{wt} \mathrm{NaCl}$ solution.

\subsubsection{Polarization Behavior}

The measured currents at different polarization levels of NPE-ZRP and ZRP coating are shown in Figure 4.27-4.28. Large cathodic current ( 2-6 mA) was observed for both NPE-ZRP and ZRP samples polarized at $-1500 \mathrm{mV}$ SCE. Comparatively smaller cathodic currents were observed for $-1100 \mathrm{mV}_{\text {SCE }}$ polarization level. The initial currents upon immersion were much larger for ZRP than for NPE-ZRP, but both transitioned to an intermediate near terminal value $\sim 0.1 \mathrm{~mA}$ after 1 day of immersion. Cathodic currents were observed for the $-900 \mathrm{mV}_{\text {SCE }}$ polarization level for both NPE-ZRP and ZRP after a shift from anodic to cathodic behavior within the first day of testing. Near terminal cathodic currents, $\sim 0.001 \mathrm{~mA}$ was observed. Large anodic currents were measured in the range of 1 to $10 \mathrm{~mA}$ for NPE-ZRP and ZRP polarized to $+100 \mathrm{mV}_{\mathrm{SCE}}$ and $-300 \mathrm{mV}_{\mathrm{SCE}}$ throughout the time of testing. In the $-600 \mathrm{mV}_{\mathrm{SCE}}$ polarization regime, a fast drop in anodic current from an initial current $\sim 1 \mathrm{~mA}$ a near-terminal current of $\sim 0.05 \mathrm{~mA}$ occurred within 30 minutes and 2.5 hours for NPE-ZRP and ZRP, respectively. 


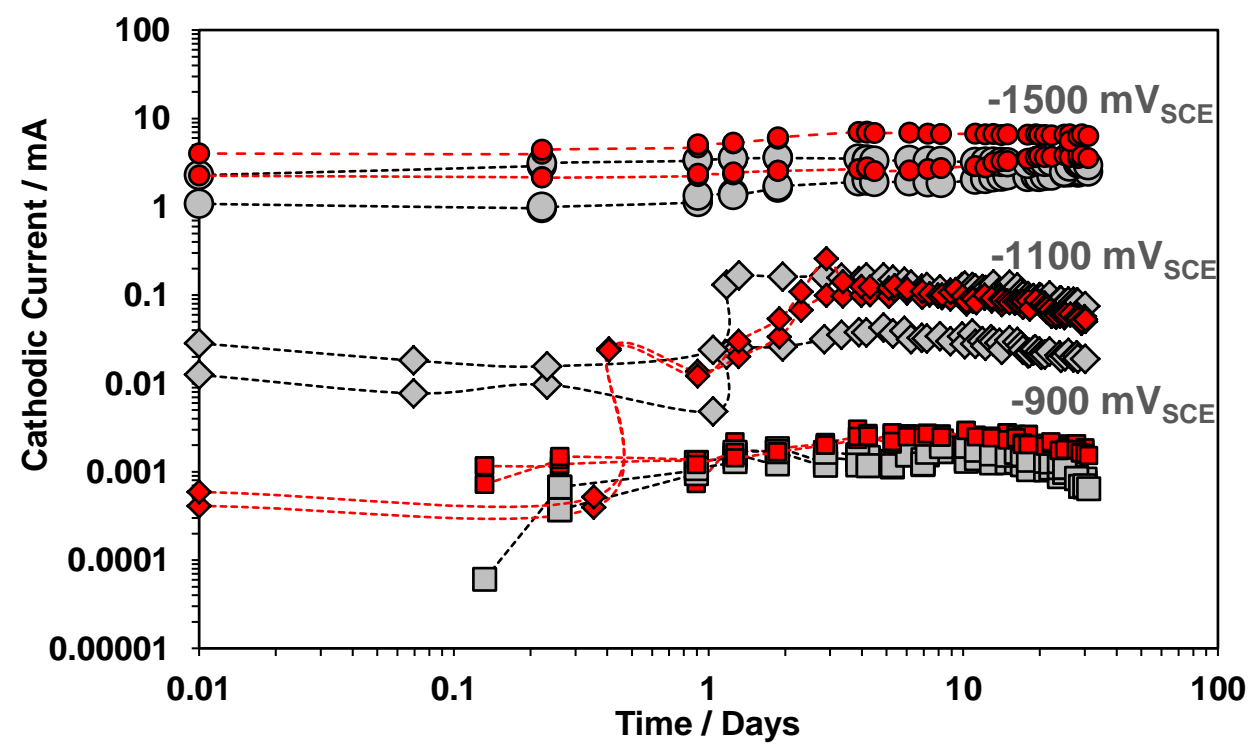

Figure 4.27. Cathodic Current over Time for NPE-ZRP and ZRP Coating.

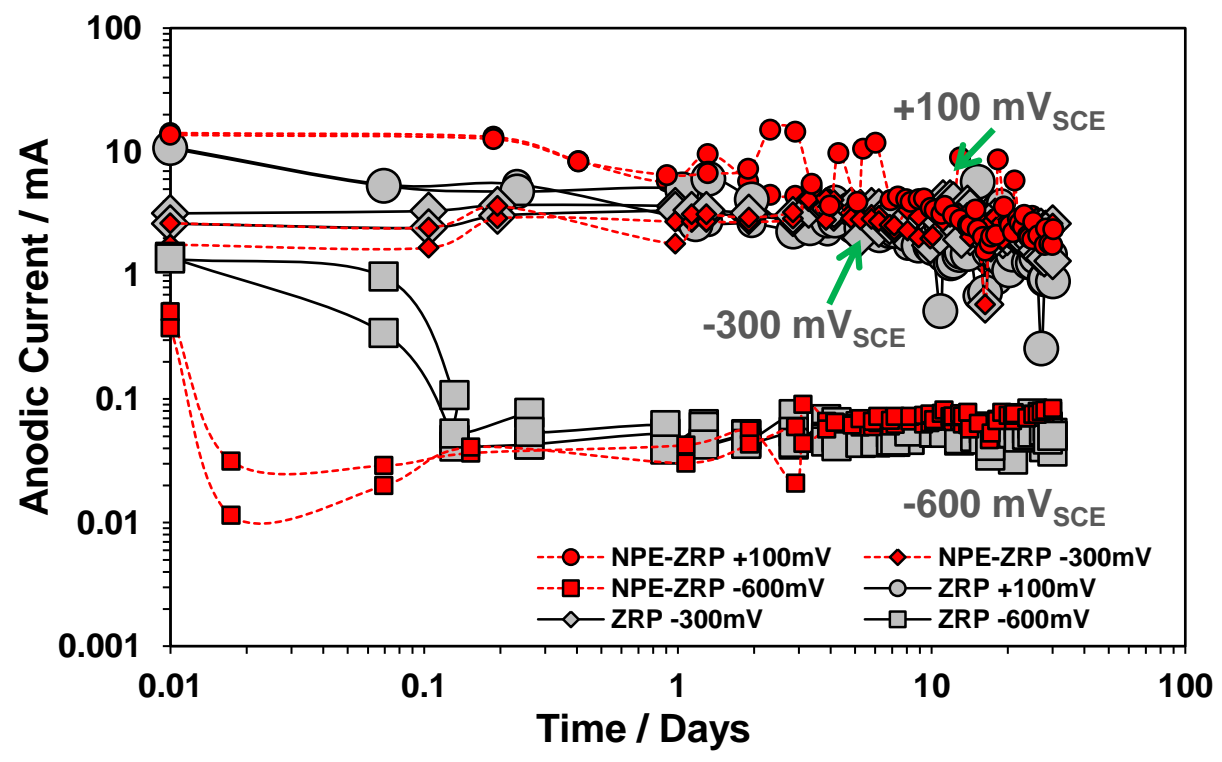

Figure 4.28. Anodic Current over Time for NPR-ZRP and ZRP Coating.

Cumulative charge plots for NPE-ZRP and ZRP coating in cathodic and anodic regimes are shown in Figure 4.29-4.30 respectively. NPE-ZRP and ZRP showed a continuous increase in cumulative cathodic charge at the $-1500 \mathrm{mV}_{\text {SCE }}$ polarization level. The large cumulative cathodic charge did not reveal any outward effects to affect the rate 
of reduction reactions such as cathodic disbondment of the coating (that could expose more steel area) or any level of zinc pigment consumption (development of zinc oxide) that would affect the rate of cathodic reaction efficiency. However, the heavy cathodic polarization would be dominated by hydrogen evolution, particularly at the coating defect site. The physical process to form hydrogen gas bubbles at a confined region on the coating defect site (and thus the mouth of possible crevices that may form due to coating disbondment) may diminish rates of reaction within the crevice itself. This may be because of the heavy hydrogen evolution reaction where hydrogen absorption can take place in the coating and because of high alkaline conditions formed at the defect site. By the evolution of $\mathrm{OH}-$, under which the zinc coating can dissolve out chemically and finally a crevice environment can form and that can facilitate the exposure of steel substrate to electrolyte underneath the coating layer, resulting in the enhancement of evolution of hydrogen atoms on the exposed bare steel surface.

At the $-1100 \mathrm{mV}_{\text {SCE }}$ polarization level, ZRP showed higher initial rates of reduction reactions than NPE-ZRP. This may be due to the greater amount of zinc pigments present around the periphery of the coating defect. There was an apparent decrease in the rate of reduction reactions there after a couple of hours, presumably due to the consumption of the zinc pigments around the defect consistent to the observed shift of potentials to passive-like conditions in the OCP samples. The NPE-ZRP did not show as much of a slowdown in cathodic activity but the amount of zinc in the periphery would be equally subjected to interaction with the solution as indicated by similar OCP testing where passive-like potentials were obtained shortly after sample immersion. On the other hand, after $\sim 1$ day, there was a large increase in the cathodic charge for ZRP which was 
thought to be related to the relatively poor mechanical adhesion of the primer in comparison to the NPE-ZRP as reported elsewhere (Saiada, et. al., 2017). It was thought that some level of coating separation in the ZRP would allow moisture access within the occluded space thus resulting in greater surface area to support reduction reactions. After a couple of days, NPE-ZRP and ZRP exhibited a similar increase in cathodic current.

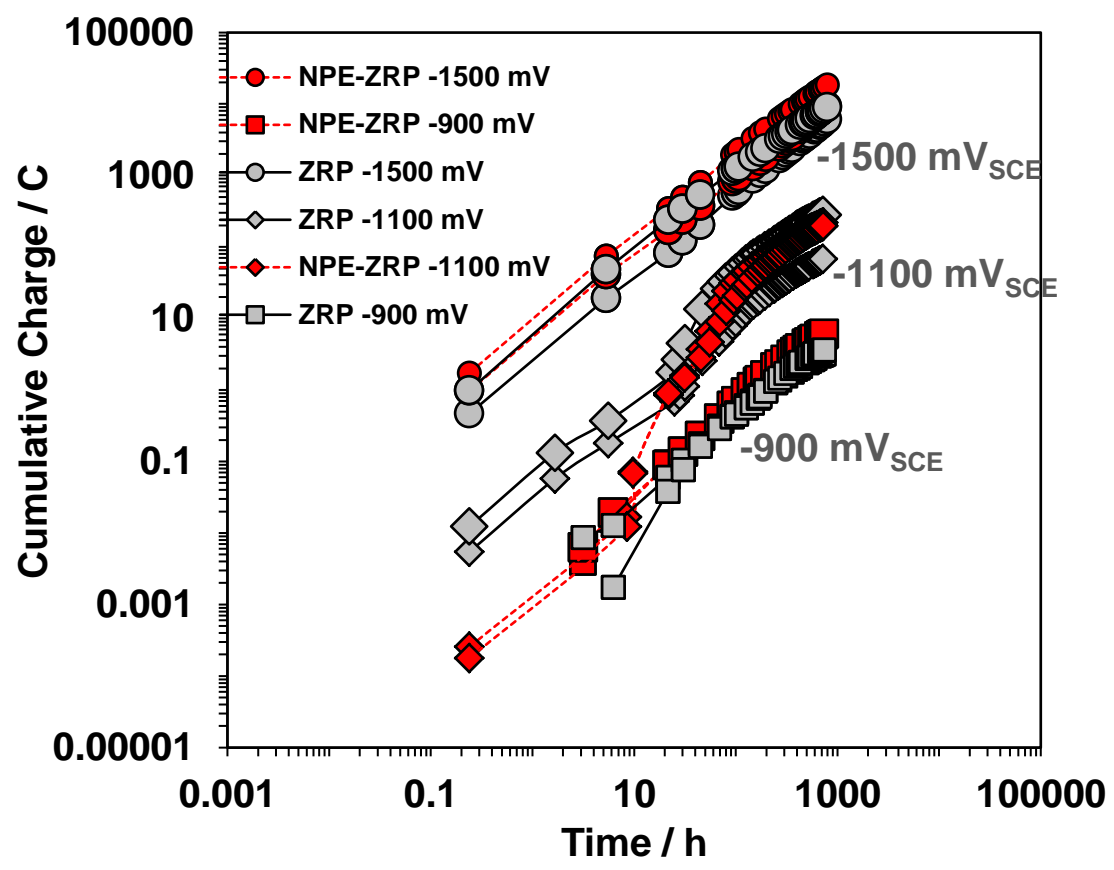

Figure 4.29. Cumulative Cathodic Charge for NPE-ZRP and ZRP Coating. 


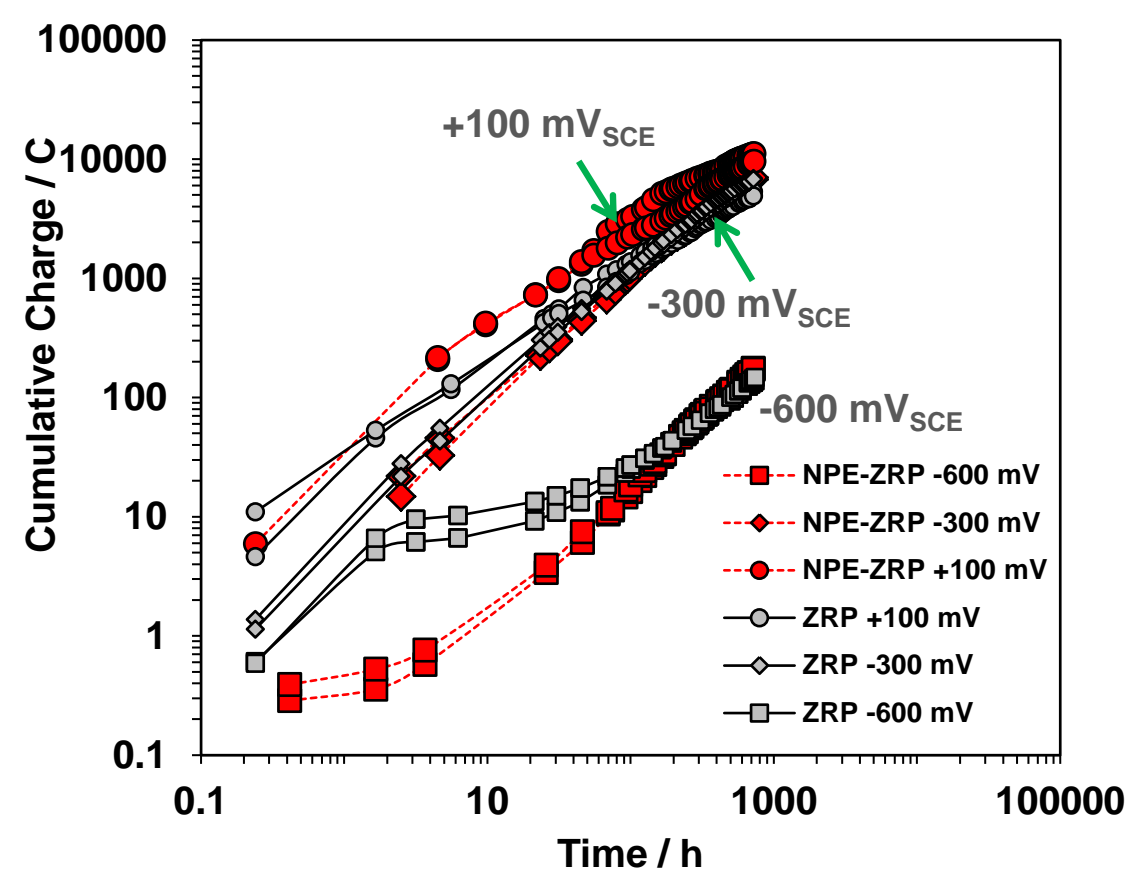

Figure 4.30. Cumulative Anodic Charge for NPE-ZRP and ZRP Coating.

At the $-900 \mathrm{mV}_{\text {SCE }}$ polarization level, there was a transition from net anodic behavior to net cathodic behavior after $\sim 2$ hours for both NPE-ZRP and ZRP coating. During the initial anodic condition, it was likely that zinc pigments were consumed. Some level of coating degradation may have occurred, particularly for the ZRP. The measured cathodic behavior after that transition would reflect those effects. Afterward, both NPE-ZRP and ZRP showed a similar steady increase in cathodic current.

At the heavy anodic polarization levels at $100 \mathrm{mV}_{\mathrm{SCE}}$ and $-300 \mathrm{mV}_{\mathrm{SCE}}$, the cumulative anodic charge for both NPE-ZRP and ZRP indicated large levels of metal loss. Severe steel corrosion was also verified by the visual observation and crosssectional micrograph as shown in Figure 4.31. The equivalent metal loss was calculated by Faradaic conversion assuming that the anodic charge is proportional to the linear density of zinc along with the depth of the coated sample and linear density of steel along 
the radius of the defect divided by the combined density of the metals along with the sample depth.

Equivalent mass loss for $-300 \mathrm{mV}$ SCE polarized samples was verified by calculating the equivalent diameter of $12 \mathrm{~mm}$ for the amount of consumption of the coated sample with the assumption that zinc accounted for a fraction of the primer based on the zinc presence density and that corrosion of the metals followed simple cylinder geometry by the steel thickness using Faradaic conversion. The calculated mass loss from the cumulative anodic charge and gravimetrically determined mass loss after exposure for both NPE-ZRP and ZRP were almost similar as presented in Table 4.2, indicating that Faradaic conversion can be used with simplifying geometric assumptions to assess the anodic activity of the zinc pigments and steel exposed to moisture with time. Slightly higher mass loss observed for the NPE-ZRP coating was apparently due to the result of the micro galvanic coupling of the interconnected zinc pigments which leads to loss of connectivity through the formation of zinc corrosion products. Due to a reduction in continuity with the zinc pigments, galvanic protection became less effective and subsequent increments resulted in steel corrosion.
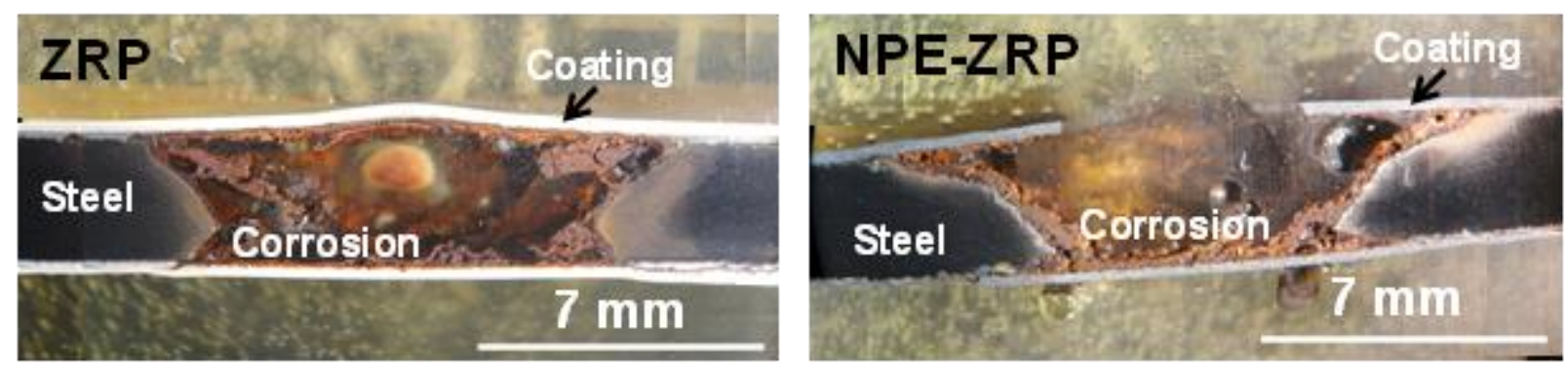

Figure 4.31. Steel Corrosion after Anodic Polarization (-300mVsCE). 
Table 4.2. Comparative Mass Loss after Exposure

\begin{tabular}{cccc}
\hline $\begin{array}{c}\text { Coating } \\
\text { System }\end{array}$ & $\begin{array}{c}\text { Polarization } \\
\left(\mathbf{m V}_{\text {SCE }}\right)\end{array}$ & $\begin{array}{c}\text { Calculated } \\
\text { Weight loss }(\mathbf{g})\end{array}$ & $\begin{array}{c}\text { Actual } \\
\text { Weight loss }(\mathbf{g})\end{array}$ \\
\hline NPE-ZRP & +100 & $2.8,3.2$ & $2.4,2.6$ \\
& -300 & $1.9,2.0$ & $1.8,1.9$ \\
& -600 & $0.05,0.05$ & $0.05,0.09$ \\
\hline ZRP & +100 & $1.5,1.4$ & $1.7,1.3$ \\
& -300 & $1.99,1.97$ & $1.8,1.8$ \\
& -600 & $0.04,0.04$ & $0.05,0.05$ \\
\hline
\end{tabular}

The $-600 \mathrm{mV}_{\text {SCE }}$ polarization case $(70-90 \mathrm{mV}$ greater than the terminal OCP values) can be considered useful to identify anodic behavior at the steady-state condition. It was apparent that the ZRP was more active in the first week of exposure compared to NPE-ZRP. The early anodic behavior for both materials was expected to be related mostly to the zinc pigments. The relatively fast anodic currents for the ZRP was thought to be due to the higher density of zinc in comparison to the NPE-ZRP, and zinc consumption was thought to be predominant at locations with the path of least resistance such as at the coating defect periphery and along the radial length from the defect. However, there was an apparent decrease in anodic current after $\sim 1$ hour, consistent with a potential shift to passive like behavior as observed in the OCP samples, which would indicate reduced effectiveness of the zinc to protect the steel. The NPE-ZRP, on the other hand, showed slower anodic rates compared to the ZRP indicating the benefit of coupling of zinc pigments within the bulk coating in the former presumably relating to the positive effect of the nano-particles. For example, the time until consumption of $\sim 5$ coulombs would occur after only 2 hours for the ZRP whereas the NPE-ZRP would have extended 
service time up to 1 day. After some level of zinc consumption when the galvanic coupling became less effective steel corrosion started for both coatings.

\subsubsection{Disbondment Test}

To quantify the extent of physical coating degradation, the radial length of coating separation around the defect periphery (including coating disbondment and blistering) was determined at the end of test exposure and was compared with the as-received conditions as shown in Figure 4.32. The radius was measured from the outer diameter of the intended defects. ZRP samples showed coating disbondment up to $8 \mathrm{~mm}$ after the cathodic polarization tests. NPE-ZRP samples showed better performance with almost no disbondment after the cathodic polarization test, further exemplifying enhanced mechanical adhesion. For the ZRP sample, coating disbondment was severe under the heavy impressed cathodic polarization $(-1500 \mathrm{mV}$ SCE $)$ and the zinc-rich primer was severely damaged up to $\sim 3.5 \mathrm{~mm}$ length from the defect site. It was evident that much of the zinc pigment underwent some level of dissolution as exhibited in the cross-section micrograph (Figure 4.33) as darkening of the zinc pigments along a large length under the coating. Zinc pigments adjacent to the coating defect in NPE-ZRP also showed similar darkening. It is posed that mechanisms that can allow coating bond degradation from the steel substrate would reduce the effective electrical connectivity of the zinc to the steel. The severe coating disbondment of ZRP under heavy cathodic polarization subsequently severed the zinc-rich primer where self-corrosion of the zinc can occur and not necessarily promote galvanic coupling to the steel. The NPE-ZRP, on the other hand, has shown evidence to promote mechanical adhesion by increasing interfacial adhesion shear strength by filling the microvoids. Cathodic polarization due to zinc activity 
(including hydrogen evolution), especially near the steel interface could then enhance coating separation and reduced coupling. Indeed, ZRP samples showed preferred zinc pigment consumption at the steel interface and not within the bulk of the coating.

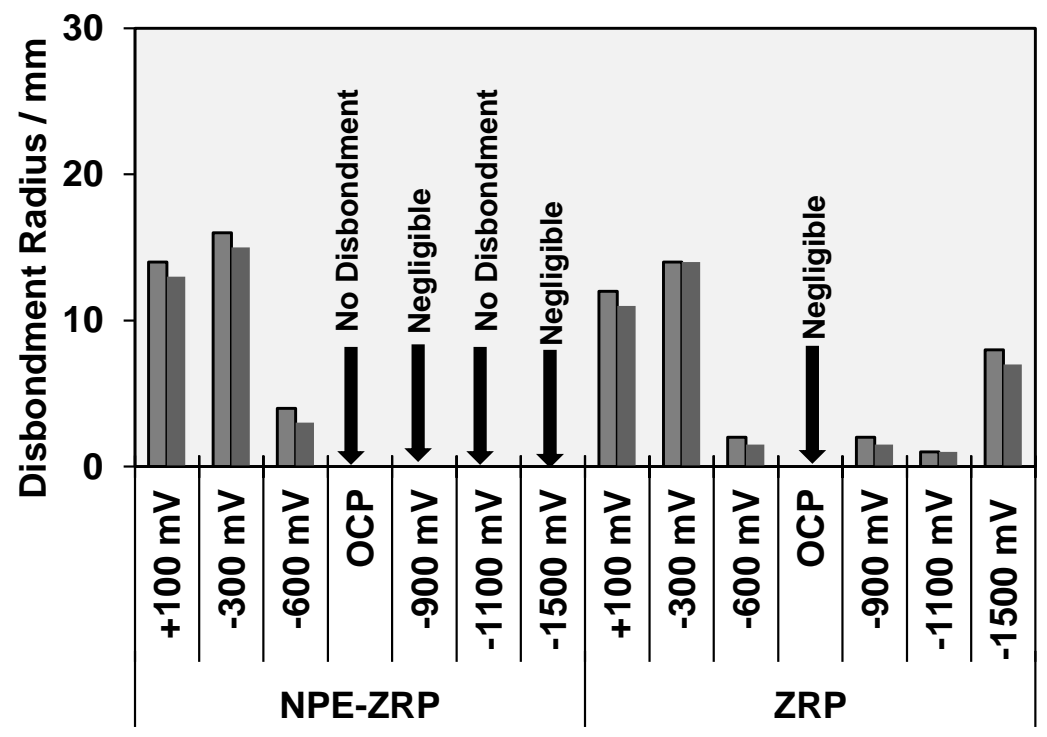

Figure 4.32. Disbondment of Samples after Exposure.

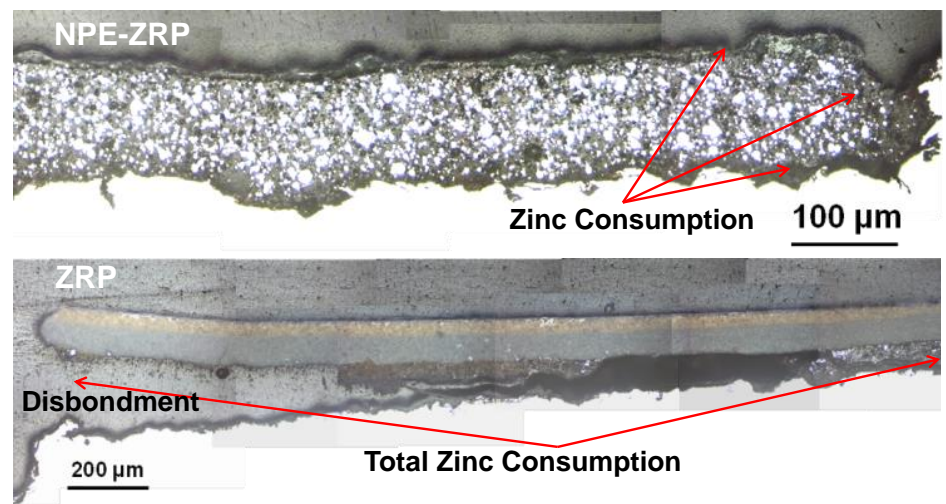

Figure 4.33. Metallographic Cross-section of Exposed Samples to -1500mV.

No coating disbondment was measured for both NPE-ZRP and ZRP coated samples exposed at OCP condition within the time period of the experiments, but longer exposures may lead to conditions as observed under the cathodic polarization tests. The coating separation from the steel substrate on heavy anodically polarized samples $(+100$ 
$\mathrm{mV}_{\mathrm{SCE}}$ and $-300 \mathrm{mV_{SCE }}$ ) was high due to the heavy amount of steel corrosion that occurred underneath by the end of the test. For samples polarized at $-600 \mathrm{mV}$ SCE, the accelerated zinc corrosion reactions due to the anodic polarization led to the further steel corrosion near the edges of the steel defect. As seen in Figure 4.34, a radial length of 2 $\mathrm{mm}$ and $1 \mathrm{~mm}$ of steel corrosion was observed NPE-ZRP and ZRP, respectively. It was evident that the accelerated zinc corrosion causing further steel corrosion allowed for the coating separation as seen in Figure 4.34. It is recalled from the cumulative anodic charge data that the rate of anodic reactions was relatively slower for NPE-ZRP than ZRP during the early exposure of more zinc pigments around the defect site. Figure 4.33 and 4.34 indeed show that zinc pigments within the bulk primer coating were active during the exposure period for NPE-ZRP whereas mostly zinc pigments adjacent to the steel interface were largely consumed for ZRP. Enhanced electrical connectivity attributed from the nano-particles would result in improved galvanic contributions from the bulk zinc pigments whereas reduced connectivity from zinc consumption in the interface in ZRP would lessen those contributions. From another perspective, the enhanced mechanical properties of NPE-ZRP would likewise allow enhanced connectivity in comparison to ZRP and provide similar results with lower zinc density. For NPE-ZRP coating zinc consumption continues through the bulk coating layer but the formation of zinc corrosion products leads to the reduction of electrical continuity for the continuous galvanic reaction. Whereas for ZRP coating zinc consumption continues along with the steel/ coating interface and the resulting zinc oxide layer subsequently blocks the electrical continuity with the above zinc pigments. In any case, after some level of zinc consumption for both NPE-ZRP and ZRP, it was evident from the cumulative anodic 
polarization curves that any beneficial galvanic coupling did not continue and then allowed for subsequent steel corrosion.

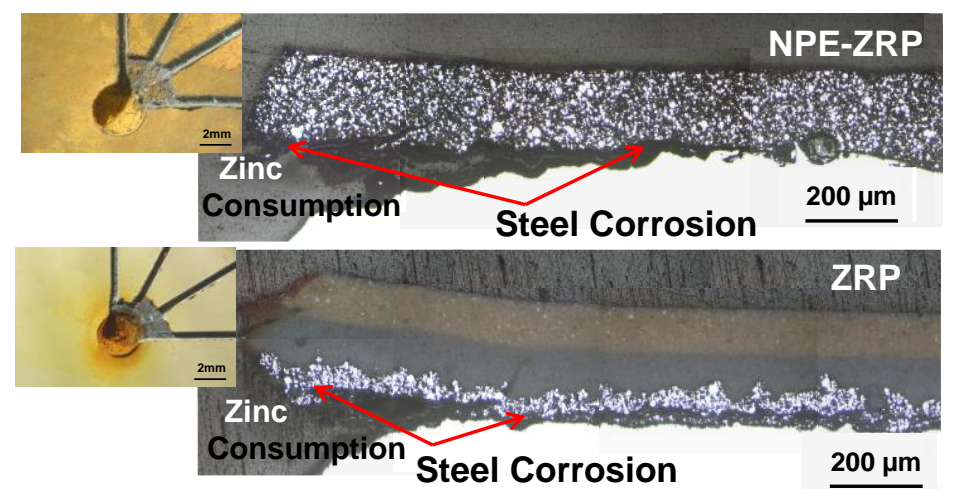

Figure 4.34. Metallographic Cross-section of Exposed Samples to -600mV.

\subsubsection{Pull-off Strength Test}

Coating degradation was also verified by the pull-off strength test. A comparison to coating in the as-received condition was made to quantify the level of degradation as shown in Figure 4.35. Higher pull-off strength values for NPE-ZRP coating was observed and compared to ZRP coating for all test conditions. For NPE-ZRP coating all pull-off testing resulted in glue failure. The results represent the minimum bound for the tests and indicate comparatively good cohesive and adhesive strength of NPE-ZRP coatings.

Higher bond strength apparently resulted from the beneficial addition of dispersed carbon nano-particles and the resulting improvement in coating property (i.e., Tensile strength, Elongation) and interfacial bond shear strength. However, the coating pull-off test after exposure gave some indication of minor coating degradation apparently due to the consumption of zinc pigments with exposure from the top surface. Anodic polarization resulted in zero pull-off strength as the coating was delaminated from the steel substrate due to severe steel corrosion. On the other hand, for ZRP coating, due to higher zinc 
content, preferable consumption occurred at the primer/ steel interface which leads to the formation layer of zinc corrosion products and further disbondment of the coating layer from the steel substrate due to poor mechanical adhesion. Zero puff-off strength of ZRP samples after exposure to $-1500 \mathrm{mV} V_{\mathrm{SCE}}$ indeed verify the comparative severe disbondment of the coating layer from the steel substrate in comparison to better adhesion of NPE-ZRP coating.

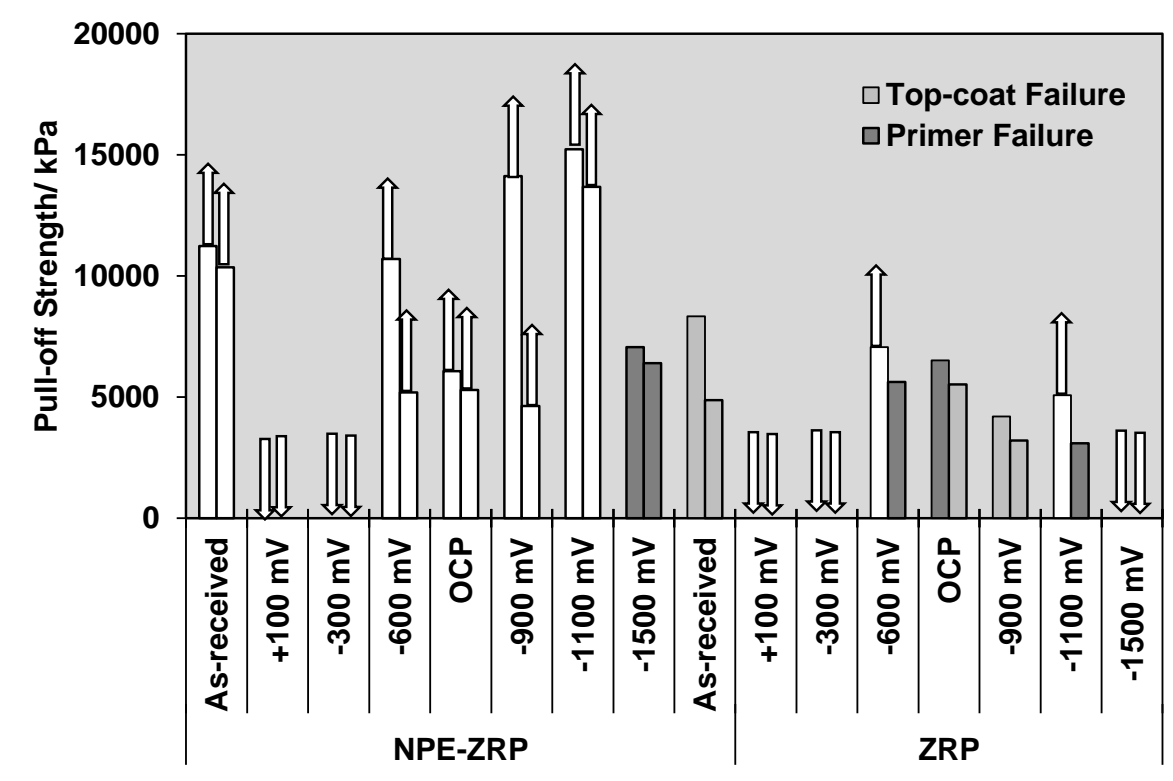

Figure 4.35. Pull-off Strength after Exposure to Various Polarization Regimes. (Arrow indicates apparent higher value)

\subsubsection{Characterization of Zinc Consumption}

XRD analysis was done at the end of the test to verify the oxidation products formed during the polarization exposure around the defect site. As received sample's result is used to see the comparative change for the exposure in the coating formulation. The white layer deposition over the cathodically polarized samples and blackish deposition over the anodically polarized was the material of interest to identify through the XRD analysis. As expected, and shown in Figure 4.36, the white deposition of 
cathodically polarized samples was the reaction product of the zinc oxidation product with the $\mathrm{NaCl}$ solution. The blackish deposition visually observed over the anodically polarized samples was mainly the iron oxide and some form of zinc oxide resulting from severe steel corrosion in $\mathrm{NaCl}$ solution.

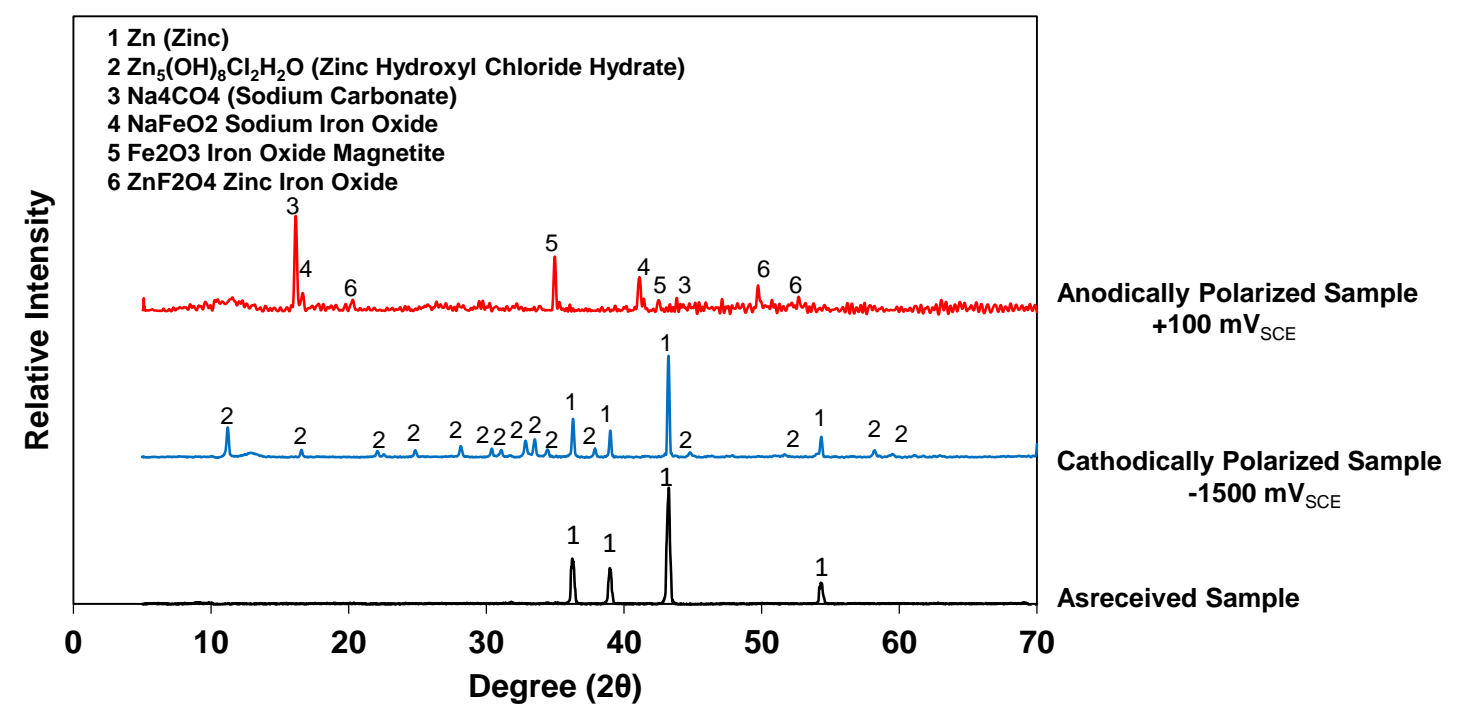

Figure 4.36. X-Ray Diffractogram for the sample after exposure.

Cross-sectional micrographs were analyzed to validate the above-discussed assertions and quantify zinc consumption. An image processing software was used to quantify the amount and modality of zinc consumption in consideration to the as-received zinc content by calculating the reduction of actual zinc area. Figure 4.37 shows the level of zinc consumption in terms of percent activity in the coating periphery of the coating defect up to $300 \mu \mathrm{m}$ length. It was seen that the level of zinc consumption in these regions was high indicating fast and early activity upon exposure to the solution. This activity was reflected by the initial electronegative potentials and early anodic rates observed in OCP samples. The behavior for NPE-ZRP and ZRP were not widely differentiated from the high early rates in this region. 


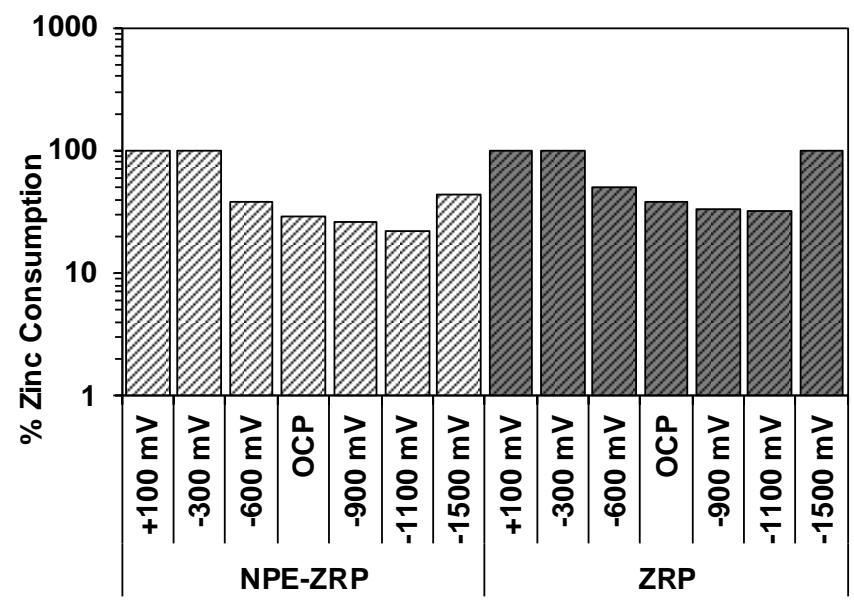

Figure 4.37. Zinc Consumption along the $300 \mu \mathrm{m}$ from Defect Site.

But the difference observed in the modality and location of zinc consumption along with the primer layer for the two different coatings. However, as observed in Figure 4.38, the zinc consumption over per unit thickness up to $5 \mathrm{~mm}$ length from the defect site was overall smaller for NPE-ZRP than ZRP for the various exposures. The overall zinc consumption was higher for ZRP coating along the 5mm length in comparison to NPE-ZRP coating for all exposure conditions implies that greater degradation of the coating system when consumption continues along with the steel/primer layer.

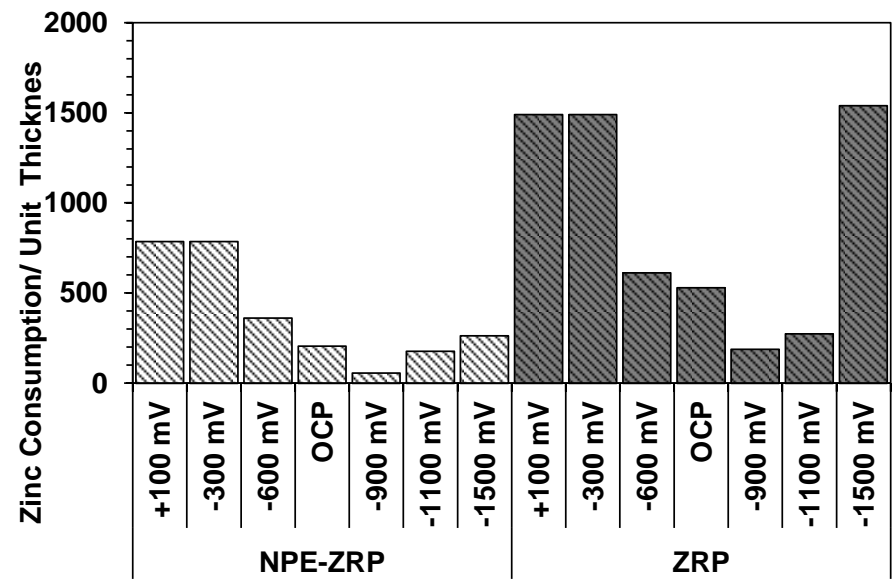

Figure 4.38. Effective Zinc Consumption per Unit Thickness along the $5 \mathrm{~mm}$ Length. 
Figure 4.39 shows the representative sample for NPE-ZRP coating where the zinc consumption observed from the bulk primer layer whereas for ZRP coating zinc consumption was concentrated along with the primer/steel interface. Initial zinc consumption was started around the periphery of the coating defect for both coatings. Dominant bulk consumption throughout the NPE-ZRP primer and major consumption along the coating/steel interface for the ZRP coating is clearly evident in the picture. Consumption along the coating/steel interface for the NPR-ZRP coating and bulk consumption throughout the primer layer for ZRP coating was also observed and apparently attributed when there was an effective continuity between the zinc pigments and with the steel substrate.

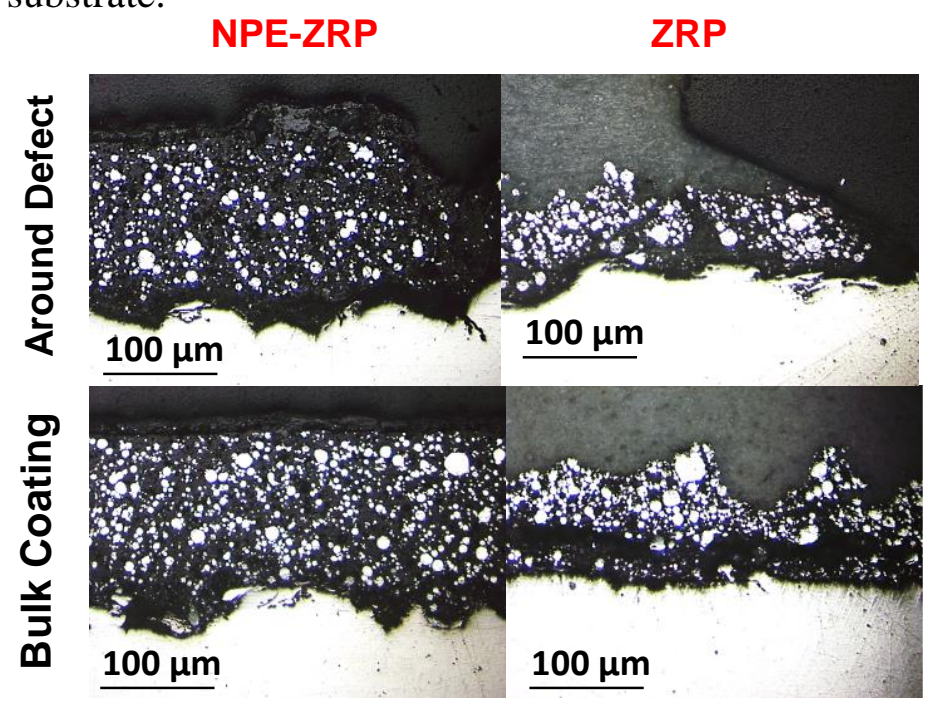

Figure 4.39. Zinc Consumption Modality after Exposure.

Table 4.3 summarize the information that quantifies the zinc consumption modality for both coatings. The data represents the area from the $>80 \%$ zinc consumption during the exposure. The consumption length clearly shows that the dominant zinc consumption for ZRP coating was along with the steel/primer interface. NPE-ZRP coating also shows consumption along with the interface whereas there was early steel 
corrosion from anodic polarization. Similar zinc consumption length for both coatings from the defect site is also consistent with the early initial activity of exposed zinc upon exposure. More significantly the total zinc consumption throughout the primer layer for ZRP coating depicts the poor mechanical adhesion and disbandment phenomena associated with cathodic polarization of ZRP coating. In contrast comparatively smaller amount of zinc consumption without any disbondment portraits the enhanced mechanical performance of NPE-ZRP coating which also observed in pull-off strength and knife test results of the coating.

Table 4.3. Zinc Consumption Characterization of Both Coatings.

\begin{tabular}{cccccc}
\hline $\begin{array}{c}\text { Polarization } \\
\text { level }\end{array}$ & $\begin{array}{c}\text { Coating } \\
\text { Type }\end{array}$ & $\begin{array}{c}\text { Consumed } \\
\text { Zinc area }\end{array}$ & $\begin{array}{c}\text { \% Zinc } \\
\text { Consumption }\end{array}$ & $\begin{array}{c}\text { Length from the } \\
\text { defect site }\end{array}$ & $\begin{array}{c}\text { Length along } \\
\text { the interface }\end{array}$ \\
\hline \multirow{2}{*}{$\mathbf{6 0 0} \mathbf{~ m V}$} & NPE-ZRP & 23792.156 & $80 \%$ & 20 & 1323 \\
\cline { 2 - 6 } & ZRP & 42833.737 & $80 \%$ & 10 & 1542 \\
\hline \multirow{2}{*}{$\mathbf{O C P}$} & NPE-ZRP & 9212.146 & $82 \%$ & 48 & 48 \\
\cline { 2 - 6 } & ZRP & 10315.66 & $82 \%$ & 45 & 235 \\
\hline \multirow{2}{*}{$\mathbf{- 9 0 0 ~} \mathbf{~ m V}$} & NPE-ZRP & 15331.323 & $90 \%$ & 20 & 517 \\
\cline { 2 - 6 } & ZRP & 7330.577 & $83 \%$ & 13 & 557 \\
\hline \multirow{2}{*}{$\mathbf{- 1 0 0} \mathbf{~ m V}$} & NPE-ZRP & 8123.32 & $80 \%$ & 25 & 106 \\
\cline { 2 - 6 } & ZRP & 12110.961 & $87 \%$ & 21 & 501 \\
\hline \multirow{2}{*}{$\mathbf{- 1 5 0 0 ~} \mathbf{~ m V}$} & NPE-ZRP & 20995.897 & $90 \%$ & 80 & 475 \\
\cline { 2 - 6 } & ZRP & 643397.8994 & $96 \%$ & 3340 & 6711 \\
\hline
\end{tabular}

All the data is consistent with the general assertion of enhanced connectivity of zinc from the bulk for NPE-ZRP than ZRP as zinc consumption at the steel/coating interface can continue within a developing crevice type environment in the latter thus allowing greater degradation along with the coating interface. Contrastingly, the enhanced connectivity of the zinc pigments in the bulk coating for NPE-ZRP allowed for zinc to interact (at least for early exposure times) to provide some level of polarization with the activity of zinc in the bulk. 
From the discussion above, it is posed that possible enhanced connectivity of zinc pigments due to enhanced coating mechanical adhesion afforded by the nano-particles would allow for greater galvanic contribution from the small zinc pigments within the bulk primer coating whereas the lack of these attributes would lead to zinc pigment consumption starting at the steel/primer interface starting at a coating defect site. These findings related to the positive effects of NPE-ZRP to promote the connectivity of zinc pigment in the bulk. 


\section{CHAPTER 5}

\section{ROBUSTNESS OF NPE-ZRP COATING FOR REPAIR APPLICATION}

\subsection{Introduction}

Nano-particle enriched zinc-rich epoxy primer (NPE-ZRP) coating was tested to identify its durability for repair coating applications and if it could provide benefit to overcome field application constraints such as surface contaminants and improper cleaning. Possible benefits as found from the application of new structural steel could be attributed either from the enhanced galvanic coupling or mechanical bonding of the zinc pigments from the primer to the steel substrate. Since the humid marine environment was expected to be a major factor during the surface preparation, moisture and salt contamination were incorporated before the repair coating application. A set of testing was made at outdoor atmospheric exposure and at alternate wet/dry cyclic exposure followed by immersing in $3.5 \mathrm{wt} \% \mathrm{NaCl}$ solution, exposure in salt-fog and exposure to the dry environment. Testing here considered localized coating damage that exposed the steel substrate. Furthermore, it was of interest to identify if nano-particles can extend the connectivity of embedded zinc pigments with improper surface preparation. In order to assess the effect of nano-particles on zinc electrochemical and mechanical coupling, steel corrosion development, the extent of zinc consumption, and coating degradation were addressed. A conventional three-coat (ZRP) was selected as control systems because these coating systems are already in use as structural steel repair coatings. 


\subsection{Steel Substrate Characterization before Coating Application}
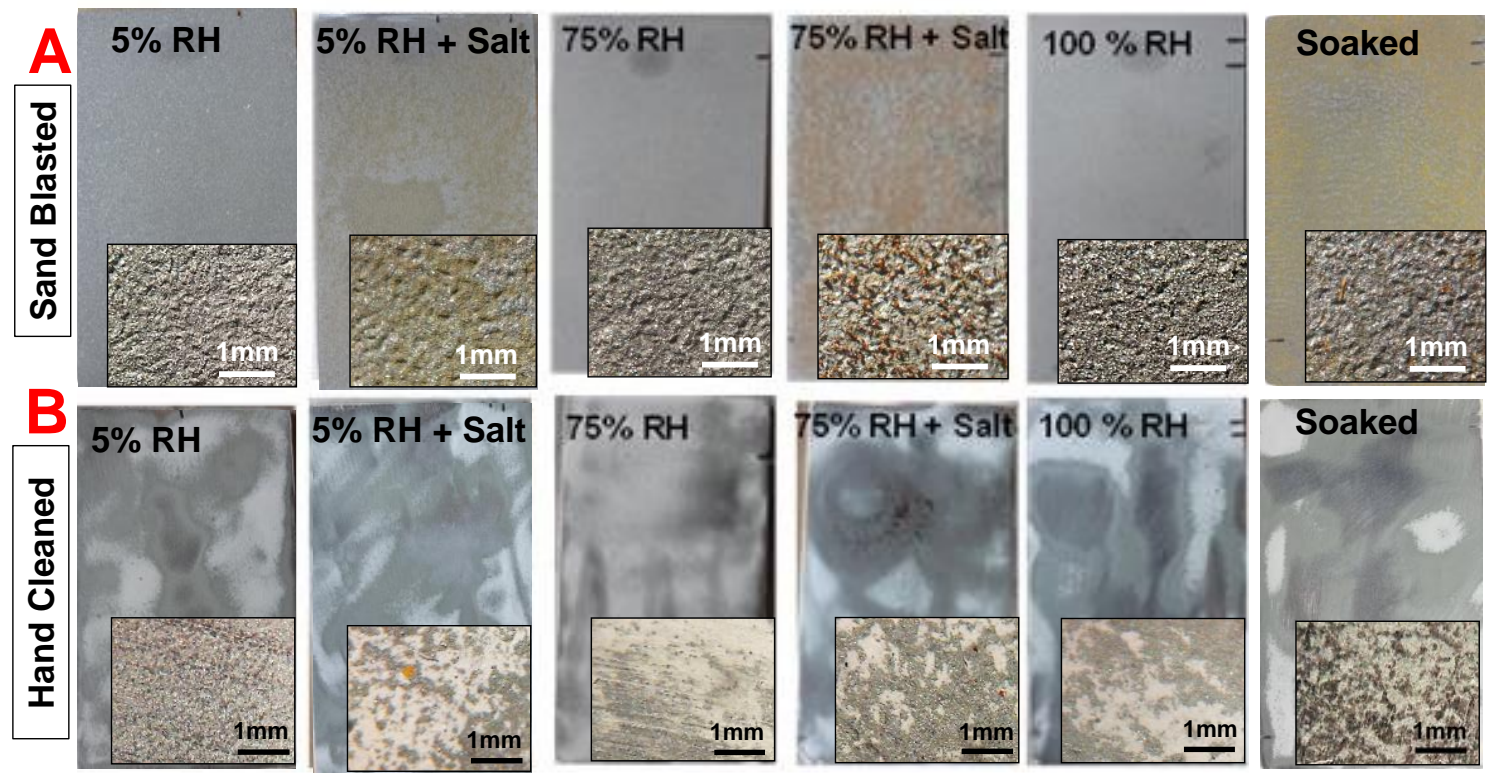

Figure 5.1. Condition of Uncoated Steel Test Panels after Exposure.

(A) Sandblasted Steel and (B) Hand Cleaned, Previously-Coated (Three-Coat) Steel.

Figure 5.1 shows the appearance of the prepared steel substrate surface after the

environmental pre-exposure. The samples with surface salt contamination showed different levels of surface changes that occurred during the pre-exposure to humidity before the application of the repair coatings. The sandblasted samples showed more sensitivity to the pre-exposure conditions than abrasion-cleaned samples. Surface oxidation was observed after the pre-exposure for all of the sandblasted salt contaminated coupons as tiny rust spots and minor rusting that formed due to the wetting and drying of chloride solution in contrast to the initially bright sandblasted finish. However, in the absence of salt contamination, the visual difference of the surface tarnishing was not distinct in the 2 days of humidity pre-exposures at 5-100\%RH levels at ambient temperatures. Unexpectedly, no visible surface rusting developed on the sandblasted coupons when solely exposed to high humidity. The initial surface oxidation that formed 
on the first day of storage in 5\% RH appeared to minimize the effect of the subsequent high humidity exposure and mitigated development of red rust. Oxidation (and rusting after the introduction of salt) was not readily observable for the hand cleaned samples where the remnant coating provided partial coverage.

Rust developed on the sandblasted samples quickly after the addition and drying of the salt solution. Prior to the 2-day exposure in the 5\% and $75 \% \mathrm{RH}$ humidity chambers, all samples had initial minor rust formation. As expected, the samples placed in the $75 \% \mathrm{RH}$ continued to rust due to the hygroscopic nature of the salt as seen in Figure5.1. Surface rust development was not observed on the abrasion-cleaned samples for similar reasons discussed above. To verify the oxide formation on the steel substrate XRD analysis was done after the environmental pre-exposure as shown in Figure 5.2. The sandblasted steel panel showed only the peaks for iron and silicon oxide. Silicon oxide was apparently available from the blast media as sand was used. Sandblasted saltcontaminated and humidity pre-exposed samples didn't show any major peak for rust formation. Humidity pre-exposed samples showed the presence of iron and silicon oxide. Salt contaminated samples also showed an indication of iron and silicon oxide. 


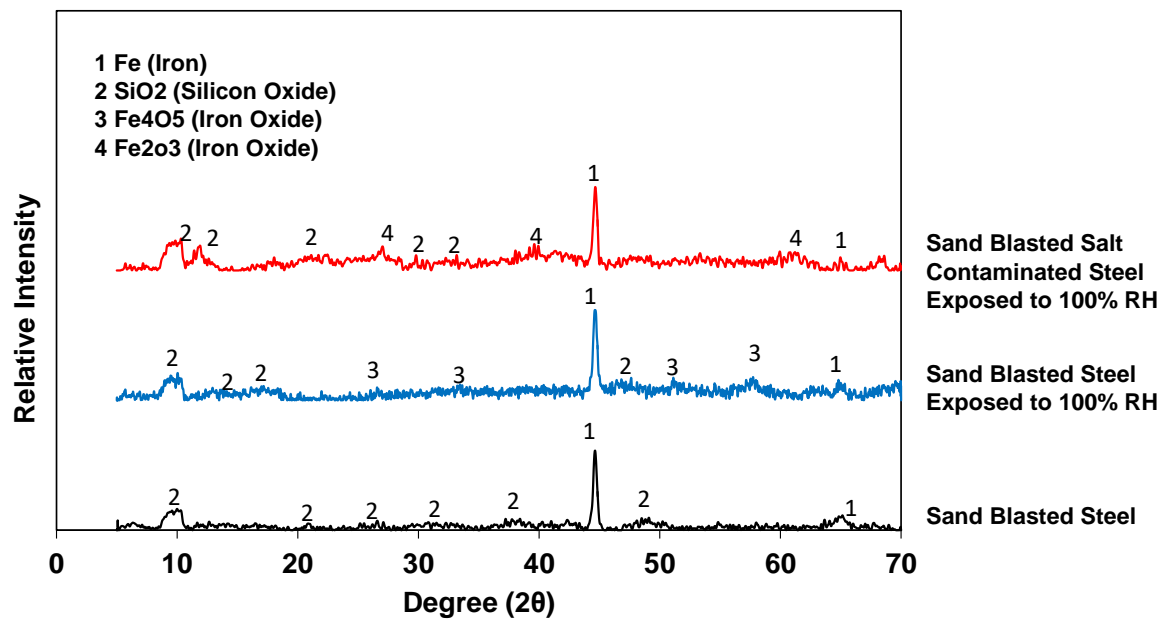

Figure 5.2. X-Ray Diffractogram for the Steel Plate after exposure.

\subsection{NPE-ZRP Coating}

\subsubsection{Material Characterization}

To identify the robustness of the material for repair application, various surface cleaning and environmental preconditioning prior to coating application was considered.

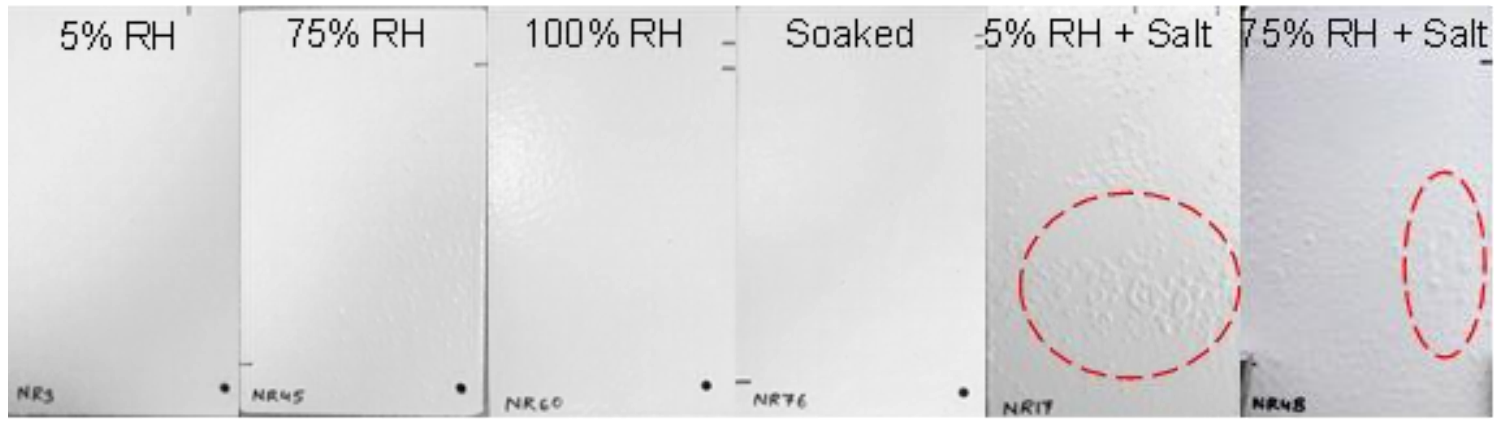

Figure 5.3. Representative Surface Condition of Repaired Test Coupons.

(Pre-exposure conditions are noted for each coupon shown)

NPE-ZRP was applied to pre-exposed sandblasted and hand cleaned coupons from different test humidity and chloride contamination exposure conditions. The effect of moisture prior to repair coat application alone to develop coating defects was nominal. Representative coating surface appearances for sandblasting and hand cleaned samples 
with exposure to moisture prior to repair application are shown in Figure 5.3. Outwardly, there were only clearly visible coating defects for the samples with either sandblast- or surface-abrasion-cleaning when the samples had pre-coating exposure to chlorides. In those cases, coating blisters readily formed immediately after repair coat application. The coating surfaces of the salt-contaminated steel coupons had an orange-peel appearance; osmotic blisters were visually identified on those particular samples in the presence of salt and moisture as shown in Figure 5.4. Osmotic blister forms due to the development of compressive stress from the expansion of permeable coating layer which allows the ingression of the water molecule to the place of less adherence of the coating layer with the substrate due to contaminants (Hare, 1984). As expected, the hand cleaned coupons retained portions of the base three-coat primer, as shown in Figure 5.5. The median thickness of the coated samples was between $\sim 250 \mu \mathrm{m}$ and $400 \mu \mathrm{m}$. The measured coating thickness for the hand cleaned coupons were accordingly higher than the sandblasted coupons, Figure 5.6. Consistent with the observed blistering for the saltcontaminated sandblast and surface-abraded samples, larger values were measured. 


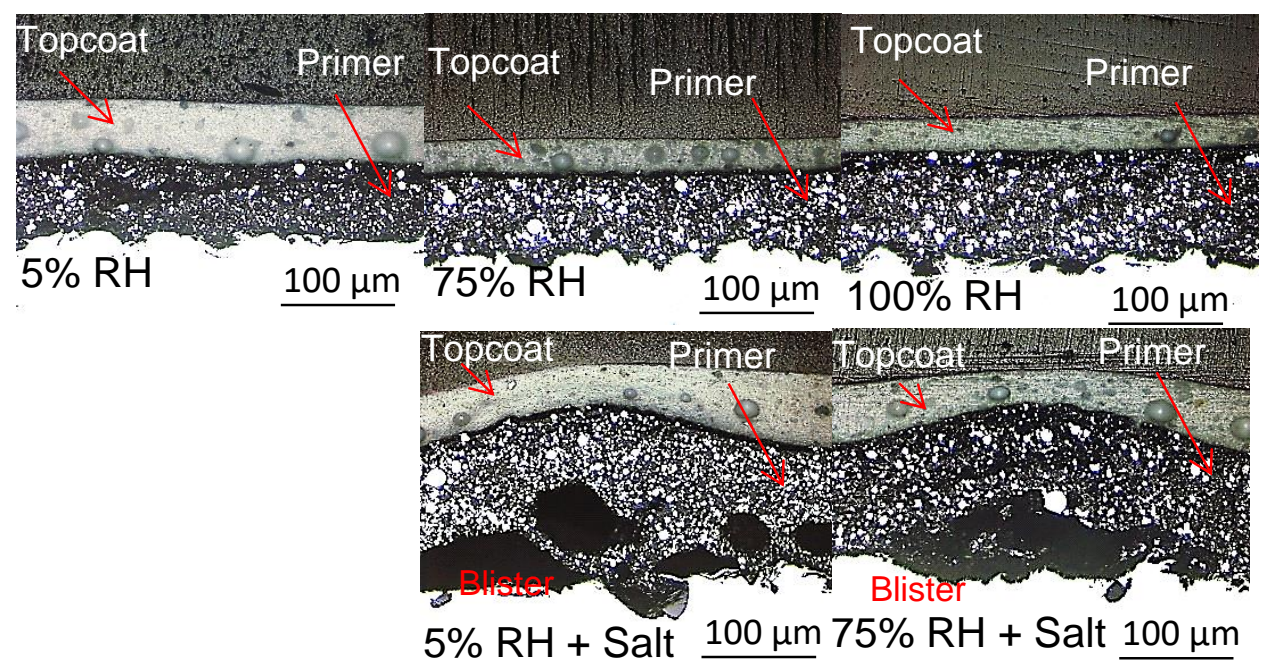

Figure 5.4. Micrographs of Repaired NPE-ZRP Samples with Sandblasted Steel. (Pre-exposure conditions are noted for each coupon shown)

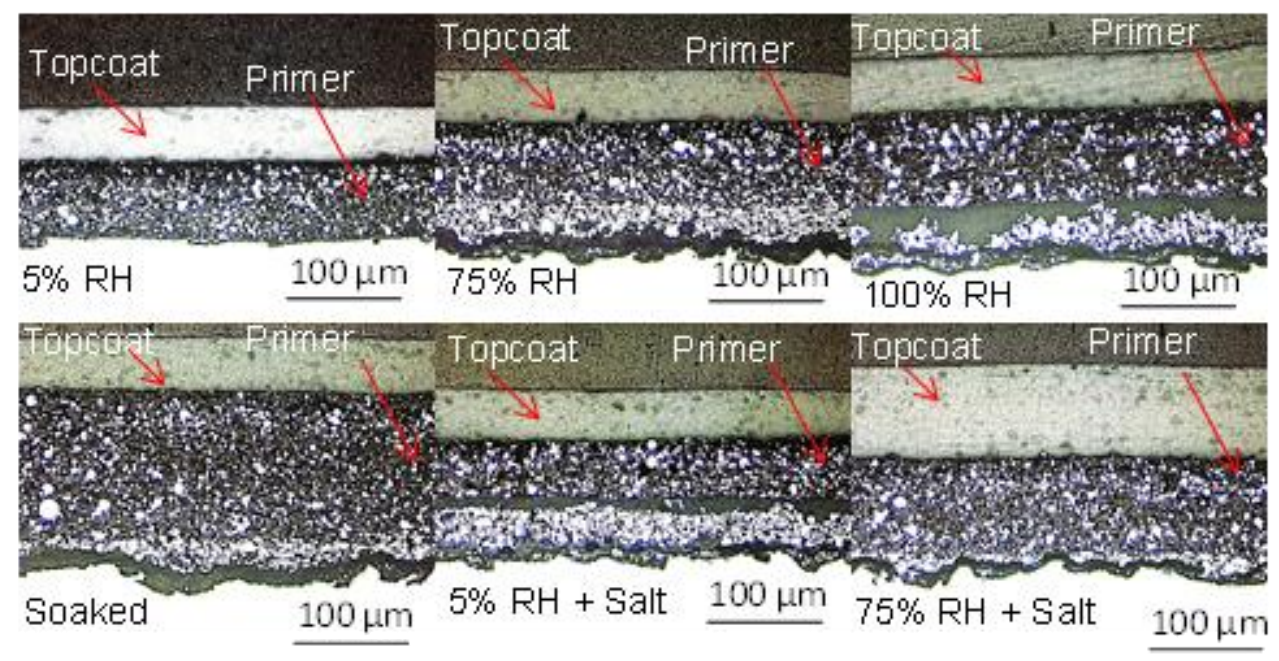

Figure 5.5. Micrographs of Repaired NPE-ZRP Samples with Hand Cleaned Steel. (Pre-exposure conditions are noted for each coupon shown) 


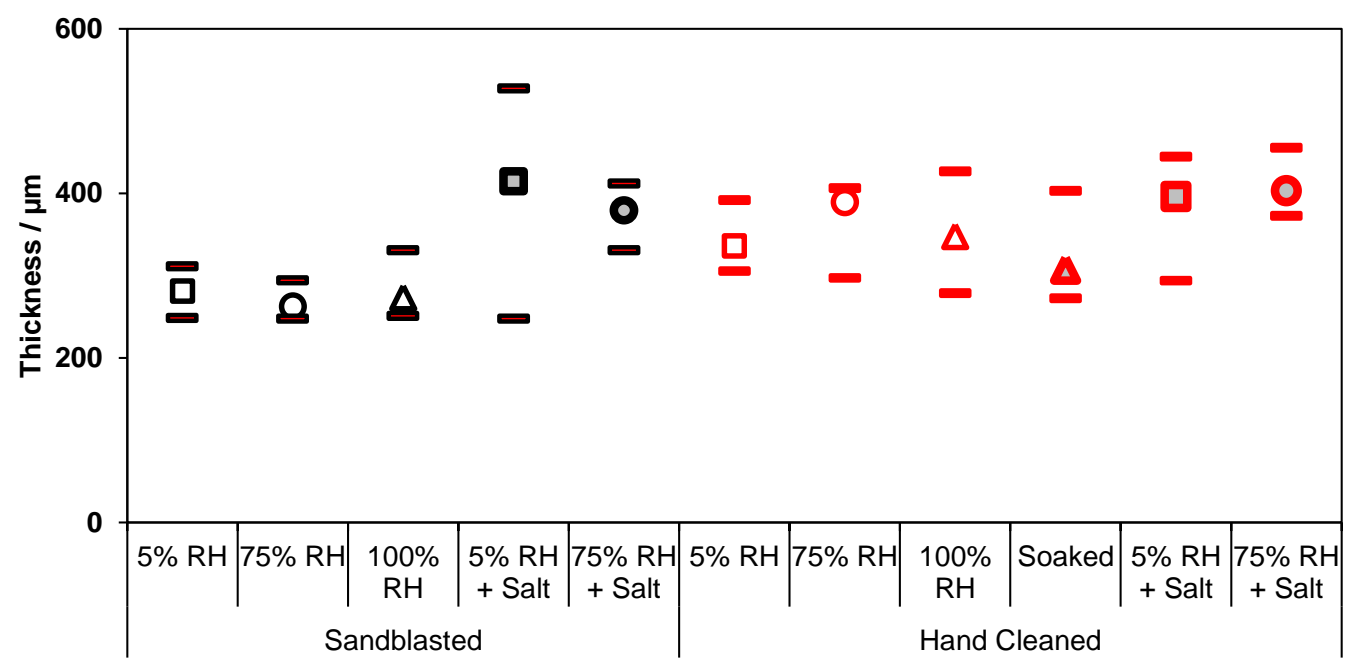

Figure 5.6. Coating Thickness of NPE-ZRP Coupons after Repair.

The coating pull-off strengths of the repaired coupons are shown in Figure 5.7.

Pull-off results were categorized as topcoat cohesive failure when both the dolly and the coupon surface had colored layers of the topcoat. The failure was considered as topcoat adhesive failure when the dolly had the topcoat material and the coupon retained the gray-colored primer. The failure was considered a primer cohesive failure when both the dolly and the coupon retained the primer. The failure was considered as primer adhesive failure when the dolly had the gray-colored primer and the coupon showed some level of grey that could be from the steel substrate, remnant mill scale, or initial surface rust. Tests that resulted in the failure of the glue to attach the dolly are shown as minimum pull-off strength. Representative pictures of different failure modalities are shown in Figure 5.8. 


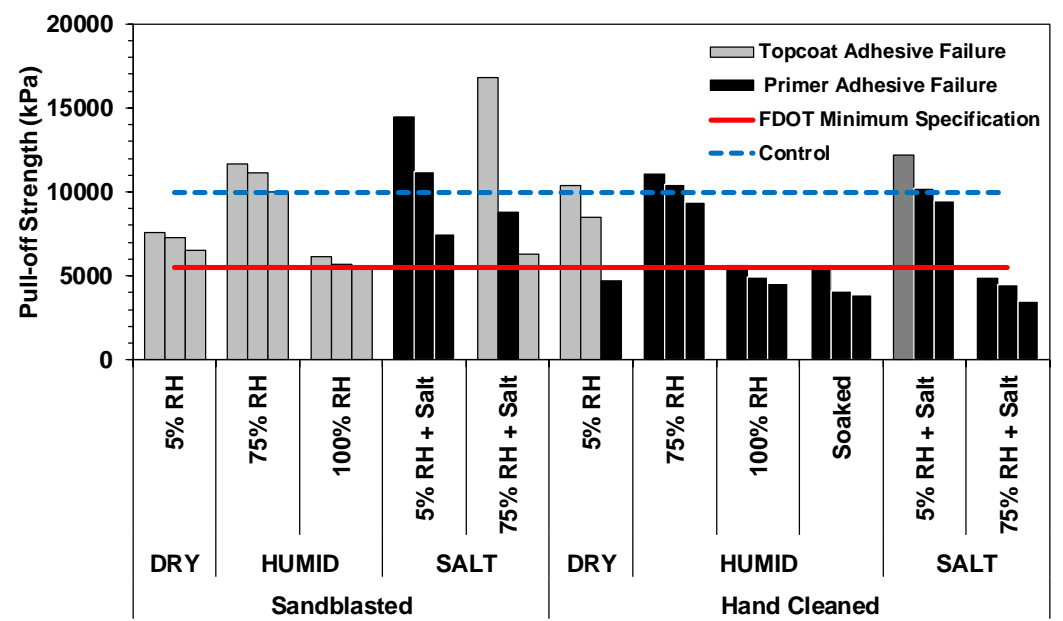

Figure 5.7. Coating Pull-off Strength of NPE-ZRP Coupons after Repair.

In Figure 5.7, the average minimum pull-off strength for NPE-ZRP applied in control condition (ideal application) and the minimum coating pull-off strength for structural steel coating systems specified by the Florida Department of Transportation (800 psi, 5,516 kPa) are shown for comparison. The NPE-ZRP coating applied in adverse environmental conditions generally showed a decrease from the control samples but still exceeded the minimum $5,516 \mathrm{kPa}(800 \mathrm{psi})$ strength requirement for all test conditions except for both sandblast and surface-abraded samples exposed to $100 \% \mathrm{RH}$ and surfaceabraded samples exposed to $75 \% \mathrm{RH}$ with salt contamination. Even though significant blistering occurred for all samples contaminated with salt, the pull-off strength testing showed values exceeding requirements for the other salt contamination test cases. For other test conditions, the coating failure modality showed adhesive primer failure (with salt contamination preconditioning and hand cleaning) and topcoat adhesive failure (with the sandblasted non-salt contaminated cases). It was apparent that the NPE-ZRP samples that were sandblasted and pre-conditioned in low to ambient moisture conditions had better adhesion than the other samples that had surface-abrasion cleaning, placed in 
high humidity, or exposed to salt contamination. It was apparent that the level of surface oxidation that formed in the preconditioning environments prior to the coating application hampered the bond between the primer and the substrate. Salt contamination, as expected, resulted in poorer performance in coating adhesion. This is consistent with the expected behavior of epoxy coatings and current application practices to use sandblast cleaning to minimize application times after surface cleaning and minimize salt exposure. After the sample preparation, which included surface cleaning (sandblast or surfaceabraded), pre-conditioning (moisture and salt contamination), and coating applicationthe test samples were exposed in environmental conditions including outdoor testing and cyclic testing.
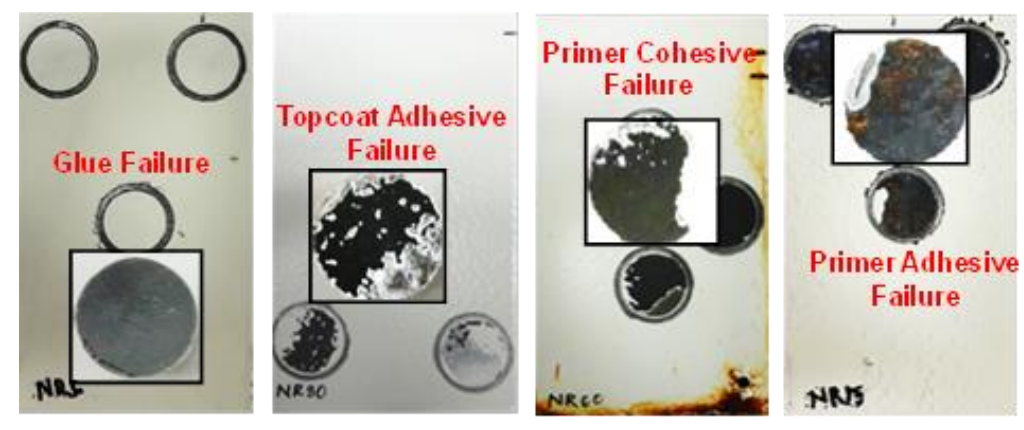

Figure 5.8. Different Failure Modality of Pull-Off Strength for NPE-ZRP Coupons.

\subsubsection{Results and Discussion}

\subsubsection{Outdoor Exposure}

\subsection{Visual}

Representative pictures of NPE-ZRP coupons after exposure are shown in Figures 5.9-5.10 (Detail in Appendix C). As described earlier, no major surface damage was apparent for the coatings except for coating blisters that formed during the sample preparation for the samples contaminated with salt. After up to 8 months of exposure 
outdoors, the samples did not show a significant change in surface appearance. The only minor surface discoloration was apparent which is apparently for the polymer degradation due to degradation by a photochemical reaction due to environmental attack (light, heat, moisture, and oxygen). Form visual appearance no difference in degradation modality was observed between the sandblasted and hand cleaned samples.

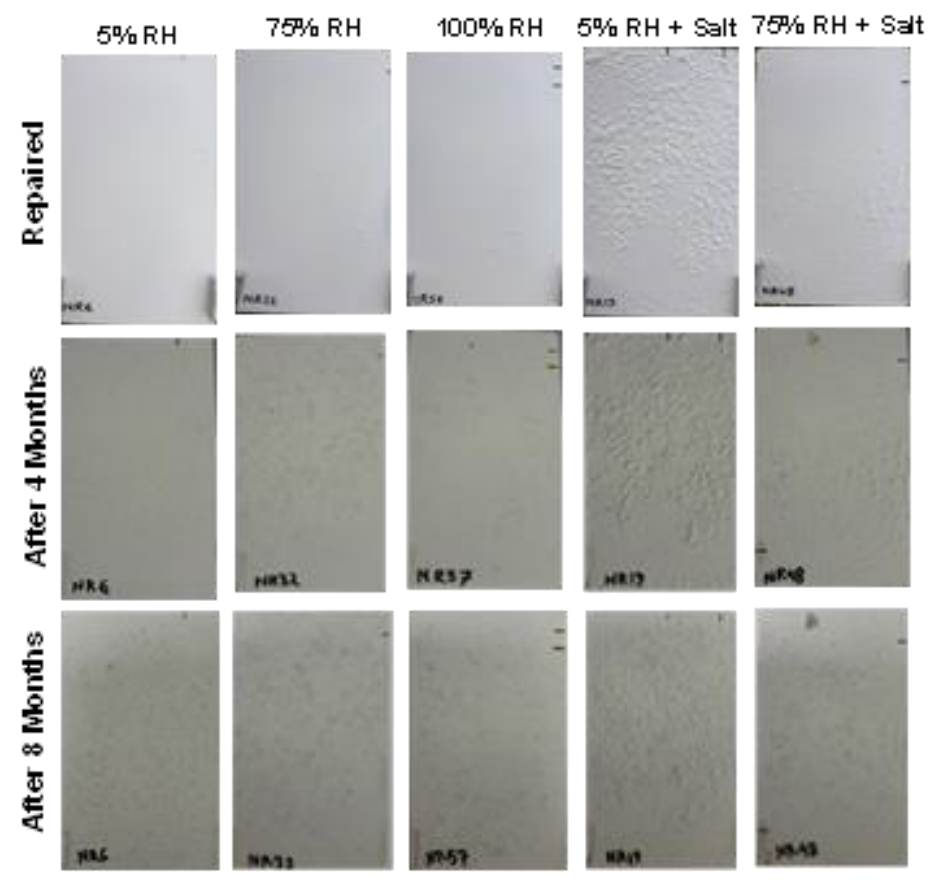

Figure 5.9. Condition of Sandblasted NPE-ZRP Samples after Outdoor Exposure. (Pre-exposure conditions are noted for each coupon shown) 


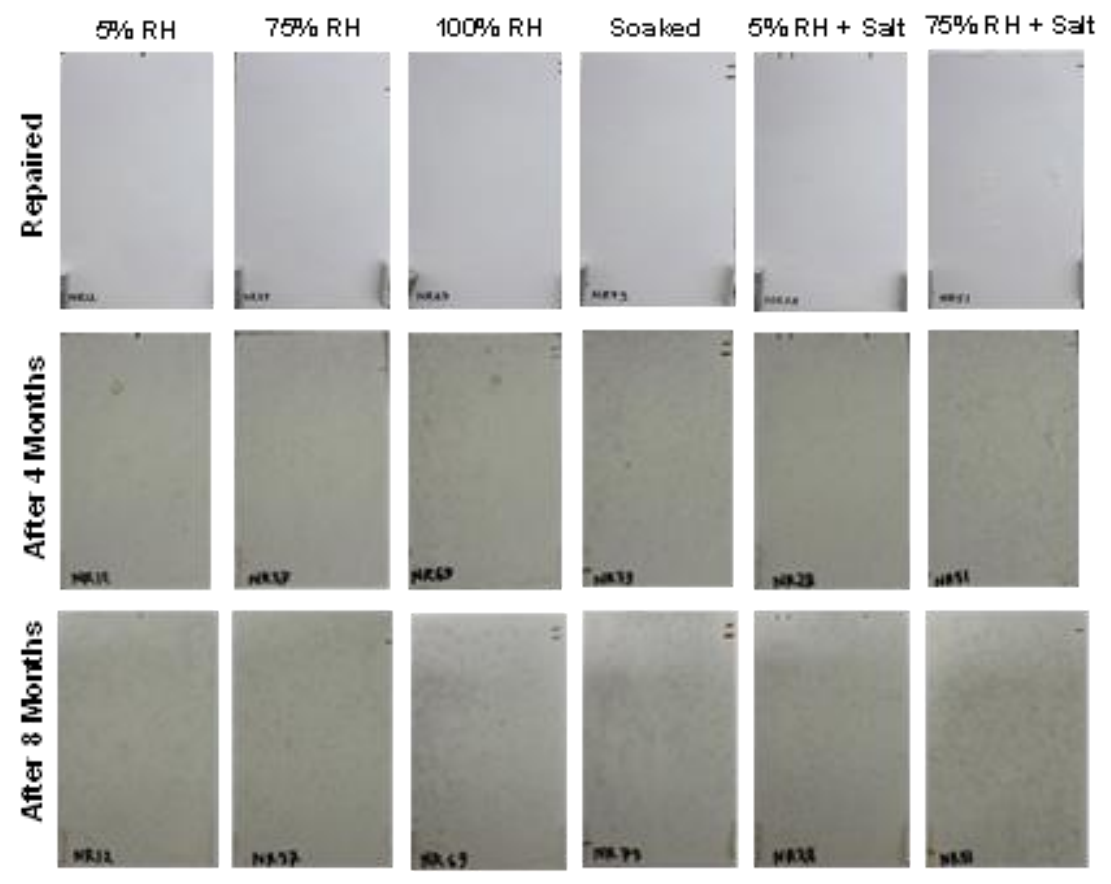

Figure 5.10. Hand Cleaned NPE-ZRP Samples after Outdoor Exposure. (Pre-exposure conditions are noted for each coupon shown)

\subsection{Coating Thickness}

As shown in Figure 5.11, in outdoor testing, no significant changes in coating thickness were measured during the 8 months of exposure. Similar trends and discussion for the sample by sample preconditioning presented earlier are relevant although some variation in the initial coating thickness was apparent. Notably, the coating thickness (repair overcoating on remnant coating layer) on the surface-abraded samples was higher than the sandblast samples and the coating thickness was higher for samples with salt contamination prior to coating application (due to osmotic blistering) than non-saltcontaminated samples. Apparent increment in the thickness for the samples with surface pre-rusting observed due to the further oxidation in those samples as oxide products occupy a greater volume than the native zinc pigment. 


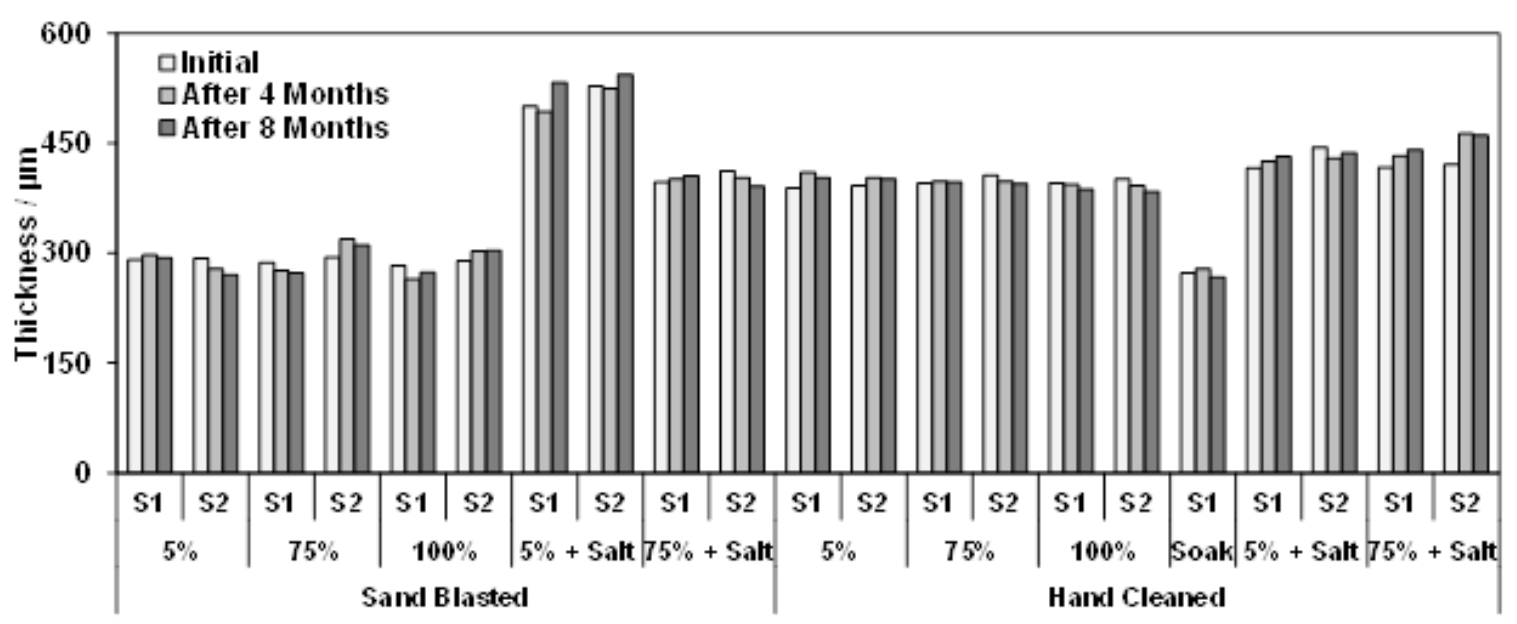

Figure 5.11. Coating Thickness of Outdoor Exposed NPE-ZRP Repaired Samples.

\subsection{Pull-off Strength Test}

After exposure, the samples exhibited some level of degradation in coating adhesion and losses in pull-off strength after outdoor exposure as shown in Figure 5.12. NPE-ZRP samples that were hand cleaned failed to meet minimum strength for all cases and primer adhesive failure was the major failure mode of coating pull-off. NPE-ZRP samples that were sandblasted failed to meet minimum strength and likewise exhibited primer adhesive failure for the chloride-contaminated cases. For the cases where the sandblast samples were exposed to only moisture, the testing typically resulted in glue failure of the pull-off dolly, but those values were still higher than for the degraded coatings. Detail of failure modality of the pull-off testing is shown in Appendix D. The minimum pull-off strength values (from the glue failure results) were mostly below the minimum required strength but the results nonetheless indicate better performance for the sandblast samples without the salt contamination. 


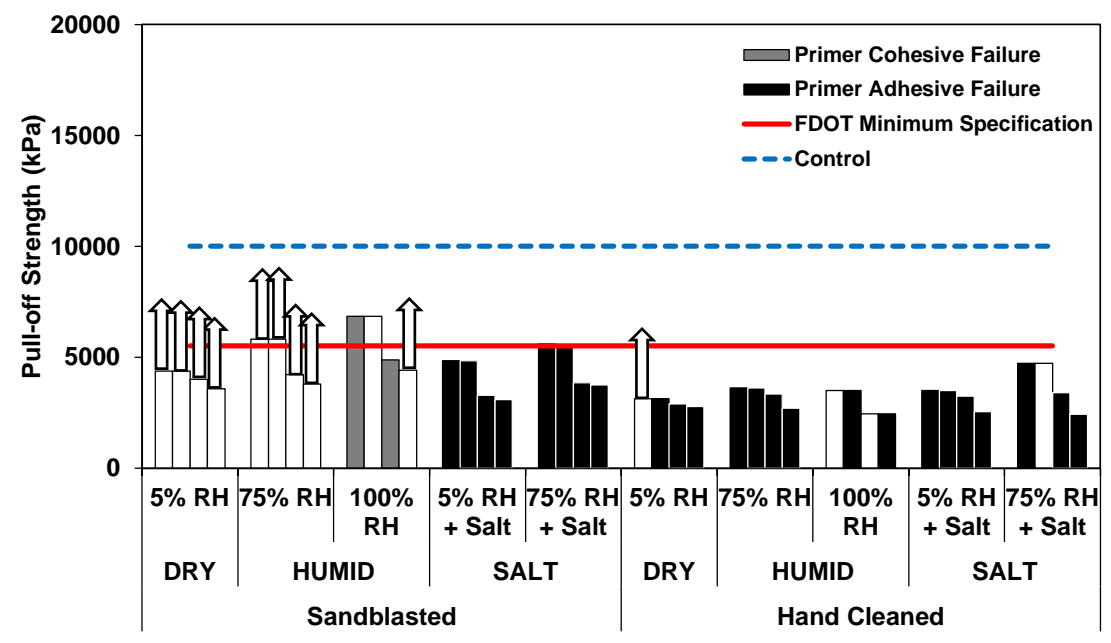

Figure 5.12. Pull-off Strength of NPE-ZRP Samples after Outdoor Exposure.

\subsubsection{Cyclic Exposure}

\subsection{Visual Observation}

Photo documentation of NPE-ZRP coupons in the as-received condition and after each exposure cycle was made. Representative photos of coated samples in the asreceived condition and after the $3^{\text {rd }}$ and $20^{\text {th }}$ cycles ( $\sim 5$ months) of exposure are presented in Figures 5.13 and 5.14, with and without defects, respectively (Detail in Appendix C). The images of the test samples with various adverse surface pre-exposure and with coating defects after cyclic exposure are shown in Figure 5.13. Rust bleed out in the perimeter of the test samples after cycle 20 was due to incidental corrosion at sample label marks on the coupon due to a lack of proper coverage on those areas. In all cases, corrosion developed at the defect site. The largest extent of corrosion developed on samples that were contaminated with salt during coating application due to the poor coating adhesion. The extent of surface corrosion even appeared more adverse for the sandblast samples than the hand cleaned samples even though similar coating blisters developed during coating application for both surface cleaning methods. The degradation 
of the salt contaminated samples apparently due to the increment in water uptake at the coating/substrate interface because of osmotic driving forces which lead to the formation of corrosion products at the interface as chloride leads to the corrosion of the steel substrate. With the time of exposure, the blister is filled with corrosion products and develops a micro-hole at the blister surface which further leads to the breaking of the blister as well as corrosion of the steel substrate as seen for the salt contaminated samples. For the non-salt-contaminated samples, no major indication of coating deterioration (other than the noted rust at defects and holidays) was observed throughout the cyclic exposure other than minor surface roughening and discoloration. The coatings appeared intact throughout the test exposure.

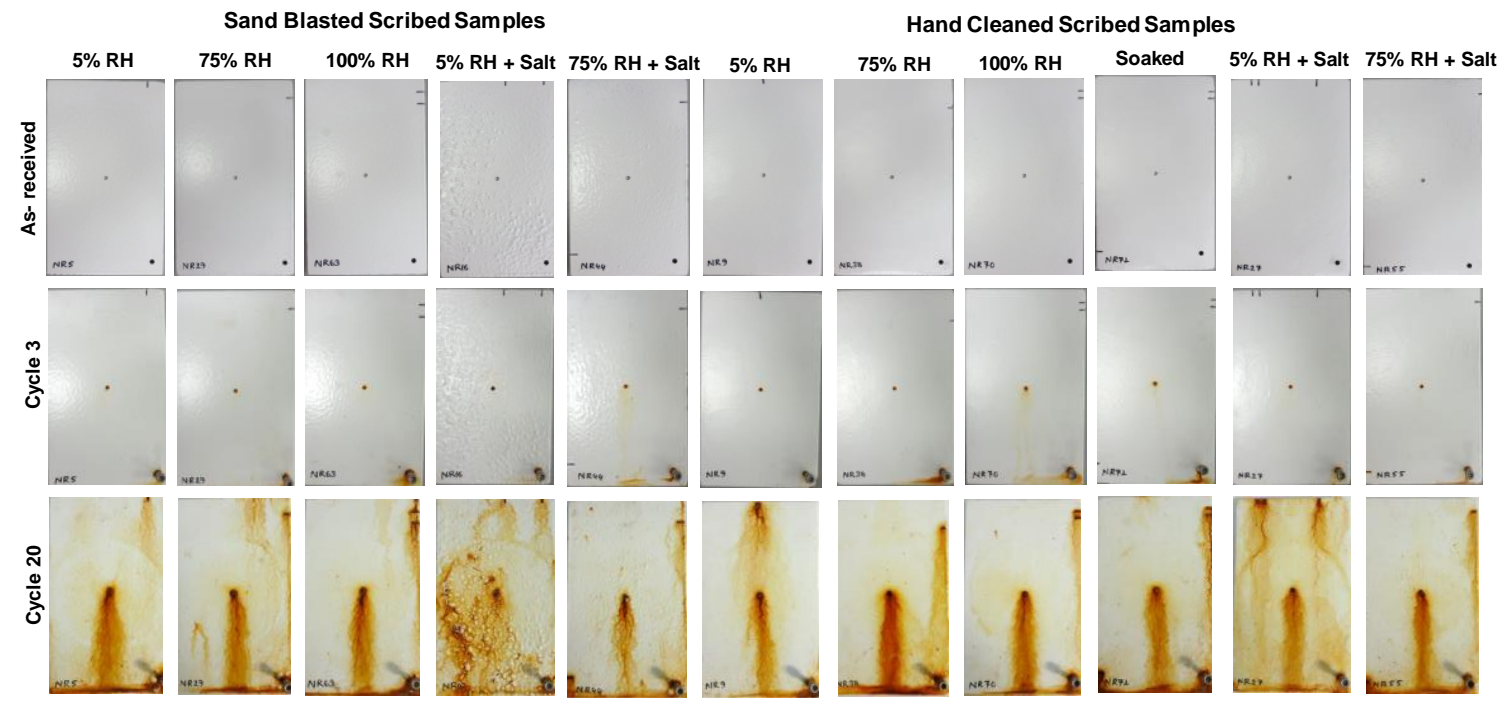

Figure 5.13. Condition of NPE-ZRP Scribed Samples after Cyclic Exposure.

Figure 5.14 shows comparative samples that did not have introduced coating defects. Like the bulk coating regions of the coupons with introduced defects, coating degradation after exposure was mostly observed for the salt-contaminated samples where the initially poor coating adhesion (due to osmotic blistering) allows for enhanced 
corrosion of the steel substrate with exposure and ingression of electrolyte. However, in the absence of local coating defects, the extent of coating degradation (although still significant) may be somewhat reduced without the easy access of moisture and salts. As mentioned earlier, a layer of zinc pigments from the original coating was retained on each of the steel substrates on the surface-abraded samples. This residual zinc layer is apparently providing some enhanced mitigation as it works as an additional barrier for the corrosive agents and also more source for cathodic protection. For example, samples with salt contamination after surface-abrasion cleaning showed less steel surface corrosion at the blister locations than samples that were sandblasted. However, the initial intact layers contained zinc pigment that was not pre-exposed. In actual service, the zinc particles in the remnant layers may not necessarily afford a similar effect if oxidized before.

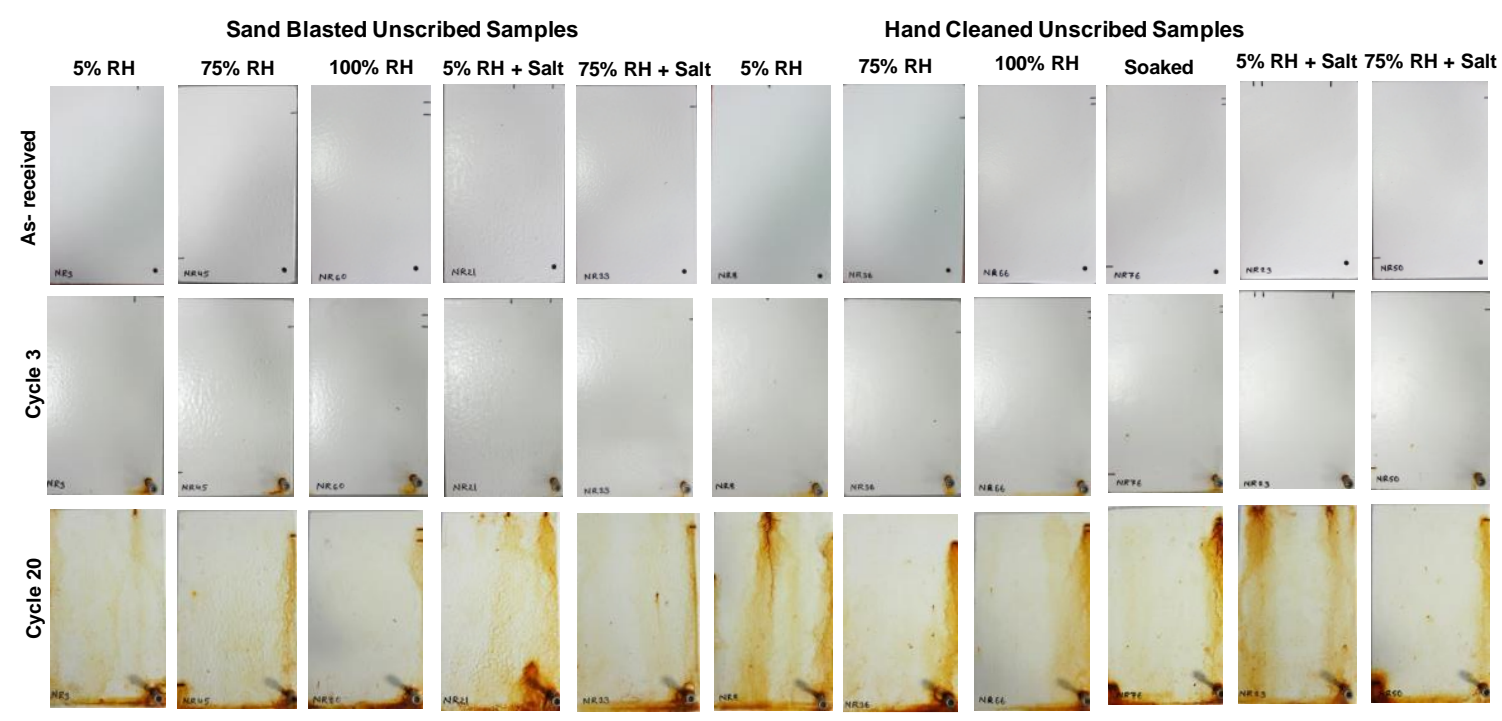

Figure 5.14. Condition of NPE-ZRP Unscribed Samples after Cyclic Exposure.

\subsection{Coating Thickness}

To identify the change with exposure, coating thickness was measured at each 
third cycle of exposure after the dry phase. Figure 5.15 represents the measured coating thickness with exposure time with different levels of humidity pre-conditioning. Coating thickness was measured for both unscribed and scribed samples to identify the effect of scribed defect. Figure 5.16 represents the measured coating thickness for samples with salt contamination.
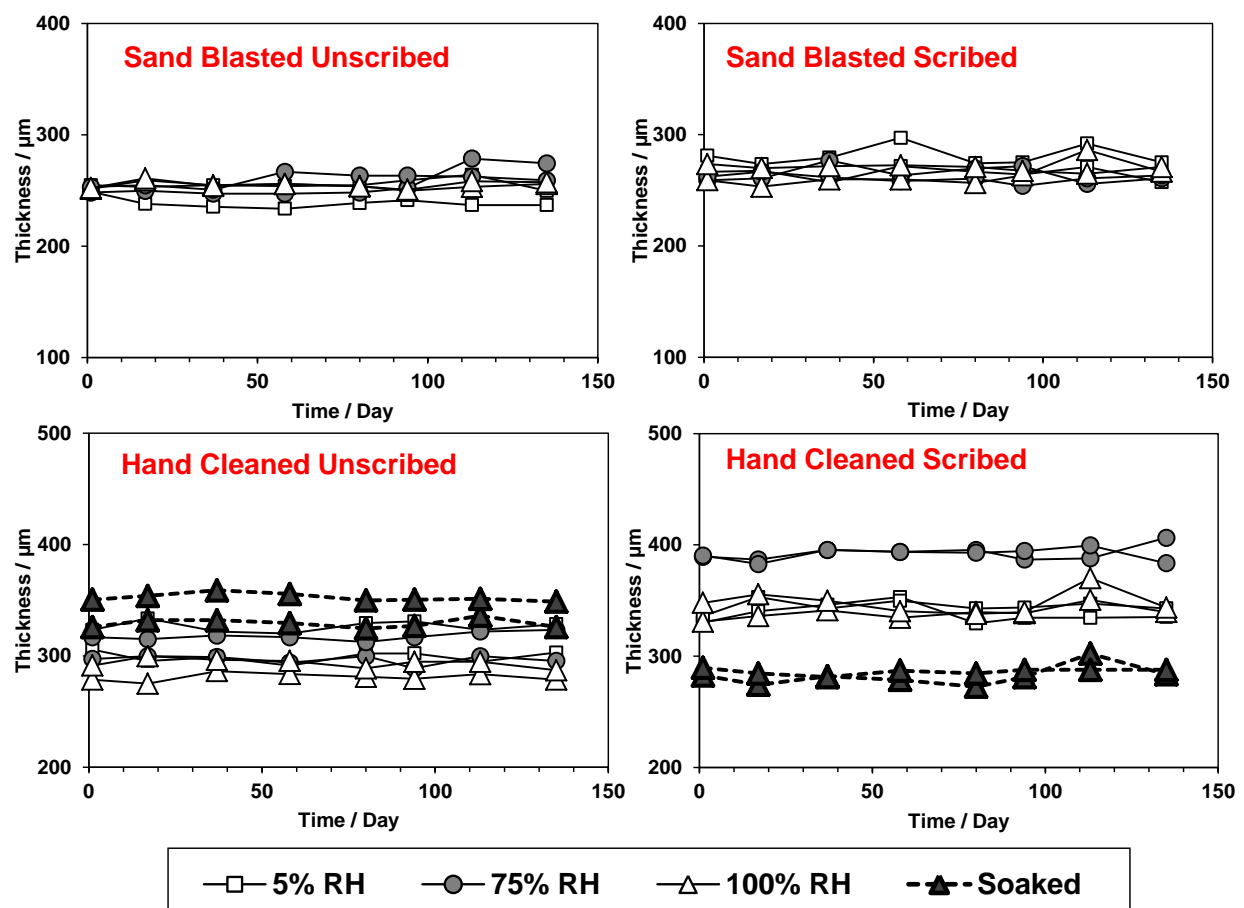

Figure 5.15. Coating Thickness of NPE-ZRP Samples after Cyclic Exposure. (Moisture Exposure during Coating Application) 

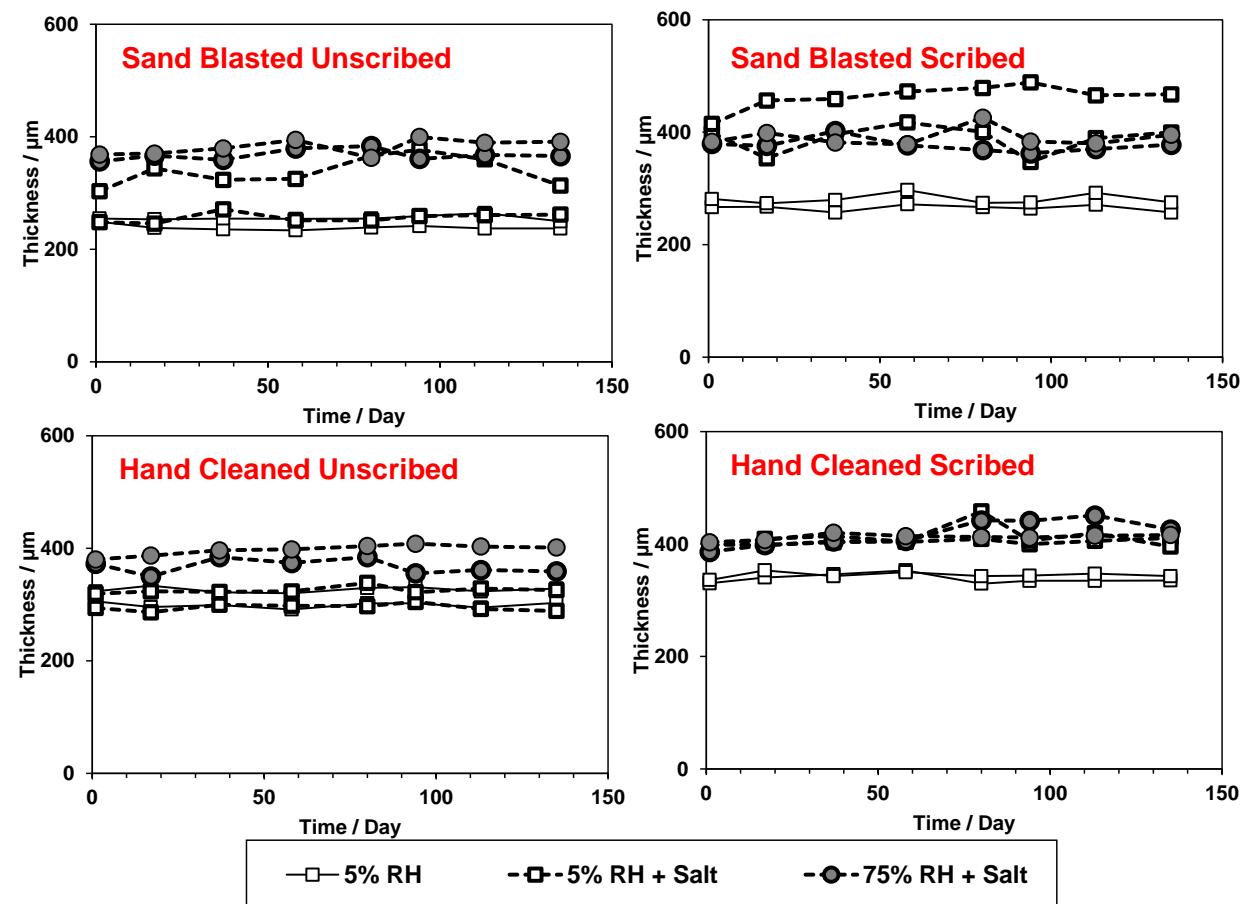

Figure 5.16. Coating Thickness of NPE-ZRP Samples after Cyclic Exposure. (Salt Exposure during Coating Application)

No significant changes in coating thickness were measured during the 20-cycle of exposure. Both unscribed and scribed samples showed similar behavior, apparently no effect of coating defect in coating thickness. Like the outdoor exposure results, similar trends and discussion for the samples based on sample preconditioning presented earlier are relevant although some variation in the initial coating thickness was apparent. The apparent coating thickness developed in the presence of osmotic blistering for the samples with salt contamination during coating application did not significantly change with the consumption of the zinc pigments and development of steel corrosion underneath. Apparent increment in the thickness for the samples observed due to the oxidation of zinc pigments with the activation by the ingression of electrolyte as oxide product occupies a greater volume than the native zinc pigment. 


\subsection{Pull-off Strength Test}

Some level of coating degradation measured as lower pull-off strength was observed after cyclic exposure; however, trends were similar to those measured after initial coating application as well as after outdoor exposure testing as shown in Figure 5.17. For those conditions, the surface-abraded samples had poorer performance than the sandblasted samples. In the former, primer adhesive failure occurred whereas topcoat adhesive failure occurred in the latter. Detail of failure modality is shown in Appendix D. However, after cyclic exposure, the surface abraded samples had lower pull-off strengths that typically were below minimum requirements. Like for the other testing, the salt contamination during coating application also reduced the pull-off strengths. Also consistent with the other testing, the effect of moisture during coating application alone did not have a strong effect on pull-off strength.

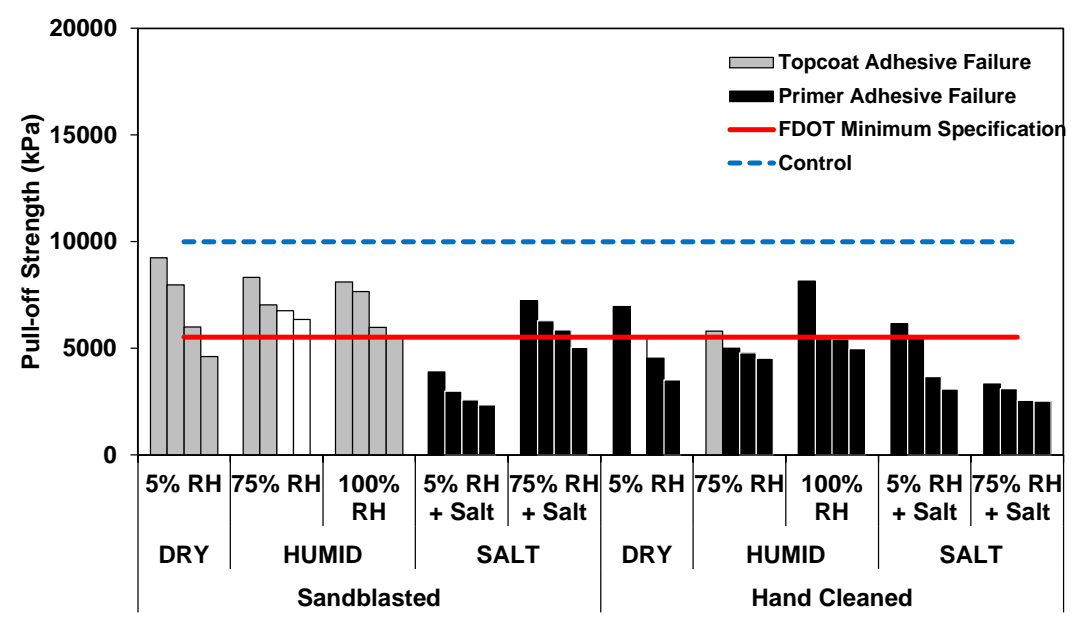

Figure 5.17. Pull-off Strength of NPE-ZRP Coupons after Cyclic Exposure.

\subsubsection{Electrochemical Analysis (OCP-LPR)}

OCP and $\mathrm{I}_{\text {corr }}$ result for unscribed and scribed NPE-ZRP samples with the various pre-coating environmental conditioning are presented in Figures 5.18-5.21. The initial 
apparent OCP for unscribed samples (Figure 5.18) was $\sim 0.0 \mathrm{~V}_{\mathrm{SCE}}$. The relatively noble potentials indicated barrier-like conditions during the early exposure times. However, with further exposure, some samples showed a drop-in potential to levels indicative of activation of the zinc pigments. The shift in the potential to a more active condition, $\sim-0.8$ $V_{\text {SCE }}$ was thought to be due to better ionic connectivity of the embedded zinc pigments to the outer test solution. This was mainly observed for samples with salt contamination during the coating application where osmotic blistering developed, but also for conditions where moisture became available in the presence of coating holidays or porous nature of the epoxy matrix. For comparative samples with intentional coating defects, the initial open-circuit potential was, as expected, electronegative ( -1 $\left.\mathrm{V}_{\mathrm{SCE}}\right)$ due to the activation of the exposed zinc pigments around the periphery of the defect sites (Figure 5.19). However, with further exposure, the potentials became more noble ( - $\left.0.7 \mathrm{~V}_{\mathrm{SCE}}\right)$ within 2 days of exposure and maintained that value to the end of the test period. The exposed steel defects should ideally be cathodically protected. However, the early shift to more noble potentials may suggest less effective protection apparently for losing of effective connectivity of the active zinc pigments with the steel substrate. Indeed, the steel corrosion was observed at the defect site after the second day of exposure. However, the terminal potential may provide some extent of beneficial galvanic coupling to the steel substrate. 

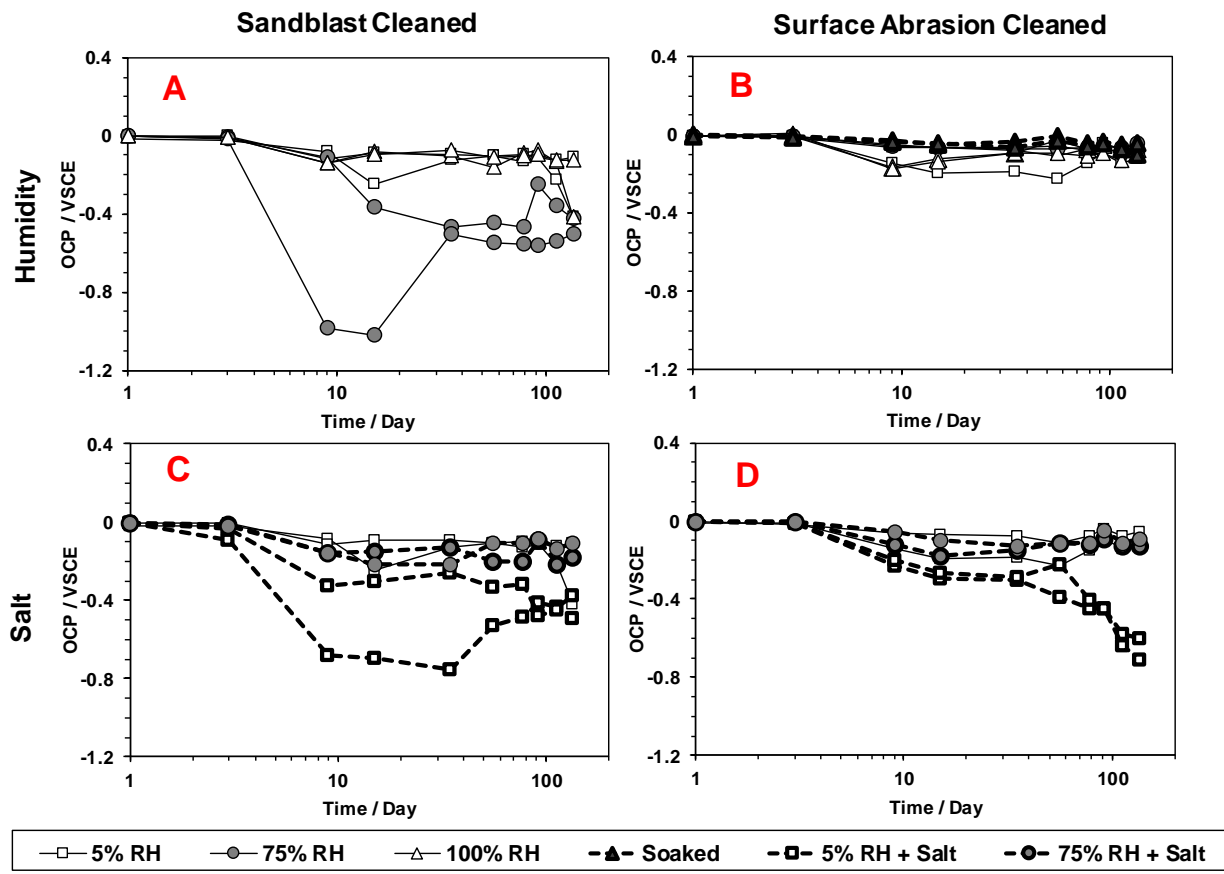

Figure 5.18. OCP of Unscribed NPE-ZRP Samples during Cyclic Exposure. A) Moisture Exposure after Sandblast B) Moisture Exposure after Hand Cleaning C) Salt Contamination after Sandblast D) Salt Contamination after Hand Cleaning
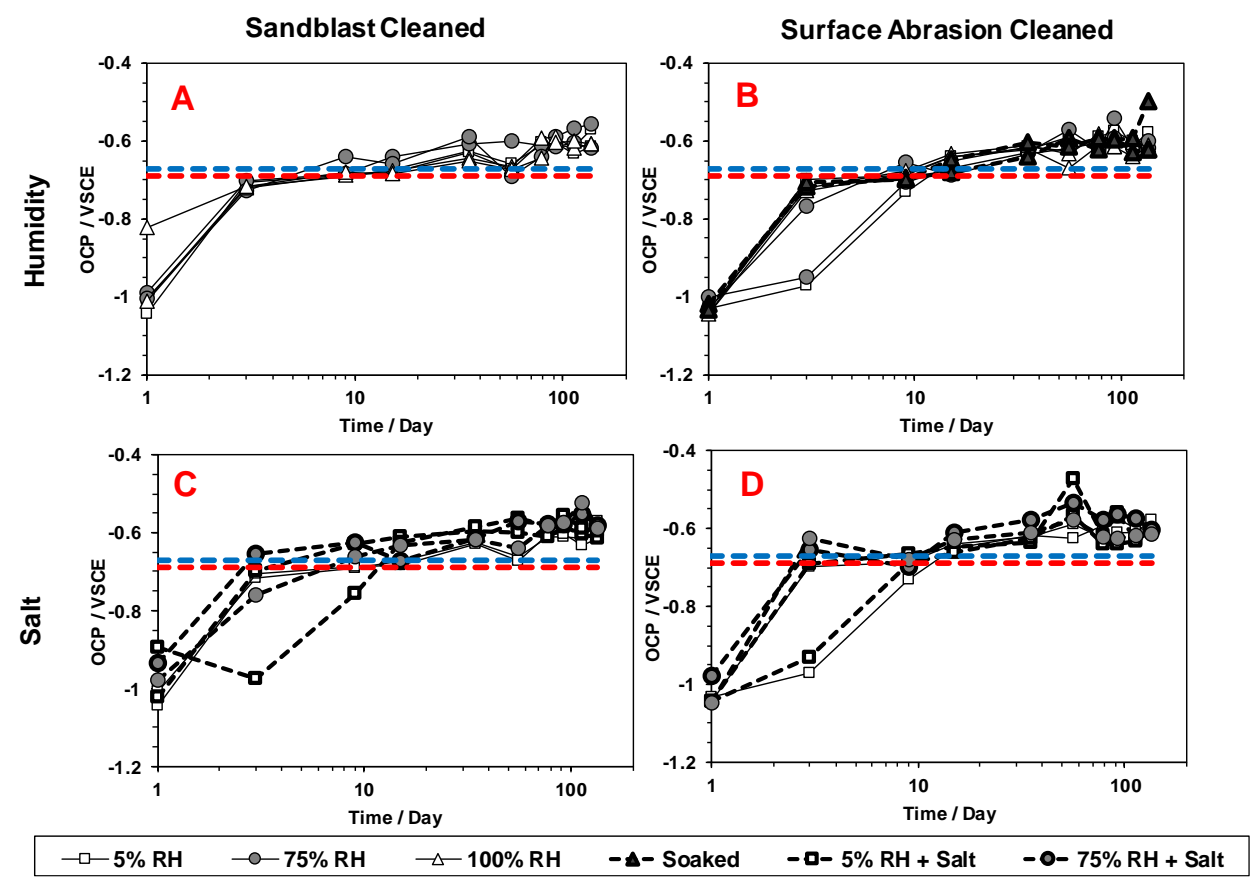

Figure 5.19. OCP of Scribed NPE-ZRP Samples during Cyclic Exposure.

A) Moisture Exposure after Sandblast B) Moisture Exposure after Hand Cleaning

C) Salt Contamination after Sandblast D) Salt Contamination after Hand Cleaning 

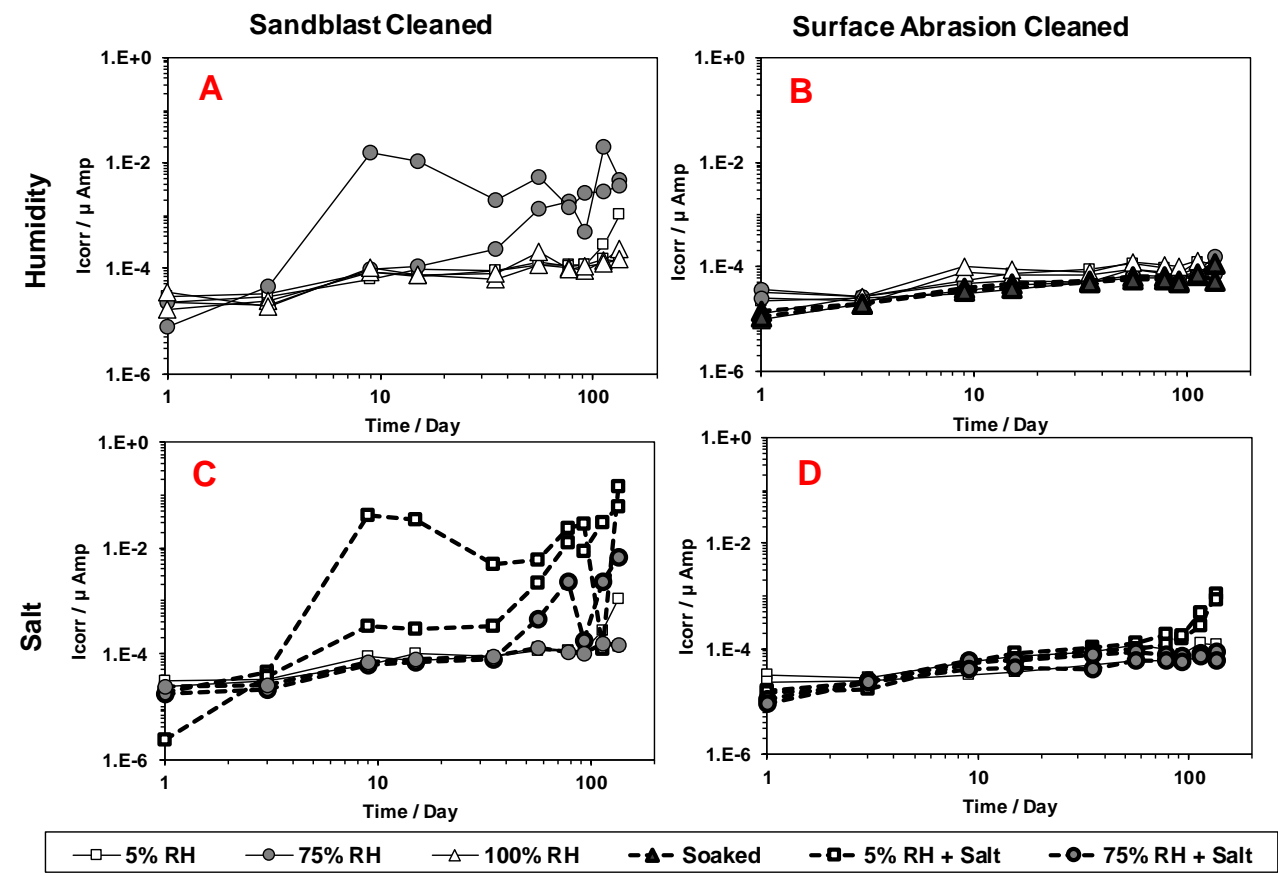

Figure 5.20. Icorr of Unscribed NPE-ZRP Samples during Cyclic Exposure.

A) Moisture Exposure after Sandblast B) Moisture Exposure after Hand Cleaning

C) Salt Contamination after Sandblast D) Salt Contamination after Hand Cleaning

The corrosion currents for the unscribed samples followed similar trends as the corresponding OCP evolution. The initial corrosion currents were very low (on the order of $10^{-5} \mu \mathrm{A}$ ) further indicating early barrier coating characteristics of the epoxy matrix (Figure 5.20). Higher corrosion rates were measured with exposure time for some samples due to the enhanced moisture presence (as discussed earlier) at coating blisters and holidays, particularly for the salt-contaminated samples as seen in the visual observation. Corrosion rates were highest for the salt-contaminated samples (as high as $0.1 \mu \mathrm{A})$. Besides the corrosion development at coating blisters due to salt contamination, the effect of moisture during the coating application was not readily apparent. For comparison, the terminal potential and $\mathrm{I}_{\text {corr }}$ of scribed sample from the testing of control samples (described in Chapter 5) are presented here as doted red (30-day immersion) and 
the blue line (60-day immersion). Similar behavior was observed for control tests, indicating the electrochemical behavior of zinc-rich primers exposed to the solution.

Corrosion currents were as expected higher (on the order of $1 \mu \mathrm{A}$ ) for the samples with intentional defects due to the exposure of the steel substrate, as shown in Figure 5.21. For comparison, the terminal corrosion current for control NPE-ZRP samples (presented in Chapter 5) were compared. The magnitude of the corrosion current for the tested samples with coating application pre-exposures and the control samples were similar at the beginning, but after extended cyclic exposure, the corrosion currents showed some indication of elevated corrosion rates. The corrosion current was relatively high throughout the test and had an apparent terminal value of $\sim 2 \mu \mathrm{A}$. This high current was measured after the apparent shift in the potential to passive-like conditions and in part accounts for the steel corrosion activity. The experimental scatter did not allow clear distinction of trends, but after $\sim$ day 50 , there was an increase in current for the sandblasted samples (particularly those that had osmotic blistering due to the salt contamination). Higher corrosion rate indicates the adverse effect of surface preconditioning prior to coating application, especially for the salt contaminated samples which also consistent with the visual observation and mechanism of blister degradation as well as corrosion of the steel substrate underneath the blister. As described earlier (and consistent with visual appearance), the comparable surface-abrasion-cleaned saltcontaminated samples with remnant zinc-rich primer showed somewhat lower corrosion currents apparently due to beneficial effect of remnant zinc layer from the previous coating application either by the enhanced barrier or by the source for more cathodic protection. With the ingression of moisture through the epoxy matrix zinc activated and 
form zinc oxide which apparently provides additional barrier protection.
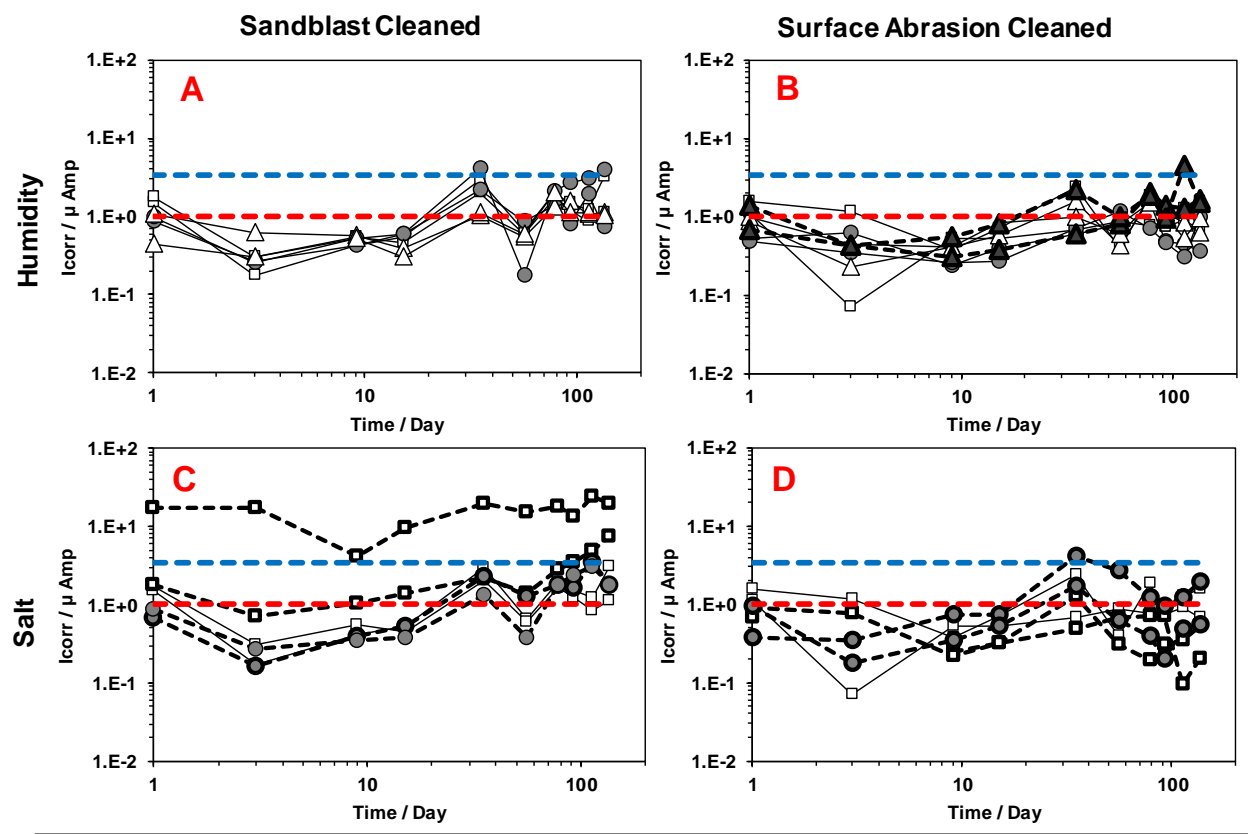

\begin{tabular}{|c|c|c|c|c|c|}
\hline$-\square-5 \% \mathbf{R H}$ & $-0-75 \%$ RH & $\triangle-100 \% \mathrm{RH}$ & - $\Delta$ - Soaked & $-\mathbf{Q}-5 \% \mathrm{RH}+$ Salt & $-0-75 \%$ RH + Salt \\
\hline
\end{tabular}

Figure 5.21. Icorr of Scribed NPE-ZRP Samples during Cyclic Exposure.

A) Moisture Exposure after Sandblast B) Moisture Exposure after Hand Cleaning

C) Salt Contamination after Sandblast D) Salt Contamination after Hand Cleaning

\subsubsection{Characterization of Zinc Consumption}

Cross-sectional micrographs were analyzed to validate the above-discussed declarations and quantify zinc consumption. Figure 5.29 shows the cross-sectional micrograph of representative sandblasted unscribed NPE-ZRP samples after the end of outdoor (8 months) and cyclic (5month) exposure in consideration to the after-repair condition. Each pre-exposure condition (before the repair coating application) is compared to identify different exposure susceptibility. Samples with different preexposure conditions showed similar behavior in both outdoor and cyclic exposure. Consistent with the sample behavior of NPE-ZRP samples for the application of new structural steel (described in chapter 5) zinc consumption was observed from the bulk 
primer layer. However, as observed in Figure 5.22, a difference was observed in the modality of zinc degradation based on the pre-exposure condition. No major degradation of the coating matrix was observed for the sandblasted, humidity pre-exposed NPE-ZRP samples other than some level of zinc consumption from the bulk primer layer, which is consistent with the pull-off strength results with comparative higher value and failure at the primer topcoat interface. This behavior is also consistent with the electrochemical results (OCP and EIS) of a potential shift to more active value and again back to nobel potential which is apparently due to the activation of zinc pigments for the diffusion of electrolyte and eventual formation of zinc oxide which is evidently displays the barrier protection with high impedance value. But major degradation was observed for the salt contaminated samples with the formation of osmotic blister which degrades more with environmental exposure. For those samples, severe zinc consumption was observed at the steel primer interface which also validates the low pull-off strength and also the significant drop of the capacitive loop of the Nyquist diagram with exposure.

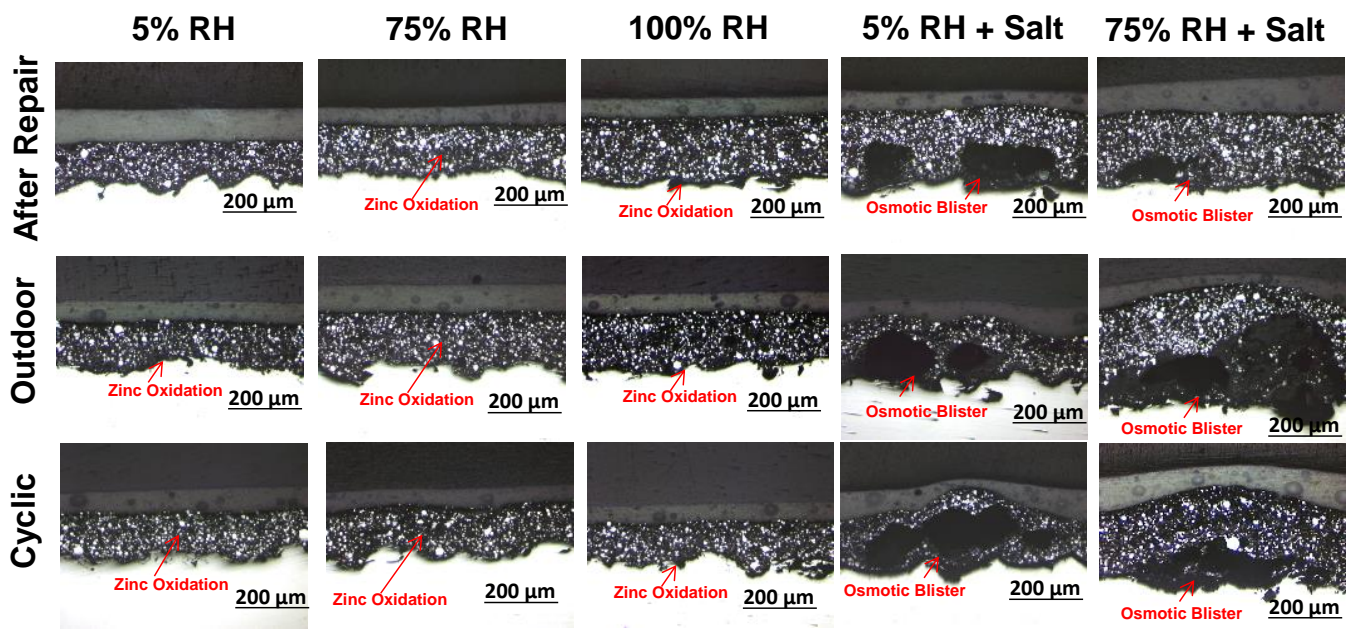

Figure 5.22. Micrograph of Sandblasted Unscribed NPE-ZRP Samples. (Pre-exposure conditions are noted for each coupon shown) 
An image processing software was used to quantify the amount and modality of zinc consumption in consideration to the as-received zinc content by calculating the reduction of actual zinc area. Figure 5.23 shows the level of zinc consumption for the unscribed samples in terms of percent activity. A similar trend of zinc consumption was observed at different exposure environment along with the different pre-exposure condition. The analogous level of zinc consumption at all different exposure portrays the consumption from the bulk primer layer. Consistent with all other findings, salt contaminated samples with $5 \% \mathrm{RH}$ pre-exposure condition degraded more with the exposure apparently due to the more absorption of moisture to the salt crystals and further reaction.

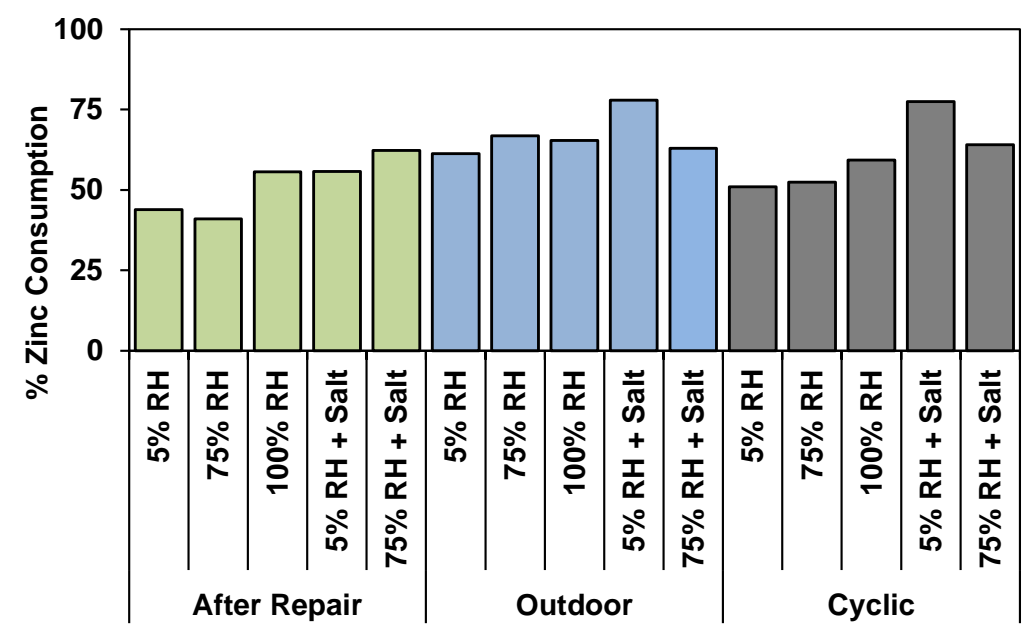

Figure 5.23. Zinc Consumption of Sandblasted Unscribed NPE-ZRP samples.

Figure 5.24 shows the cross-sectional micrograph of representative hand cleaned unscribed NPE-ZRP samples after exposure along with the after-repair condition. Each pre-exposure condition (before the repair coating application) is compared to identify different exposure susceptibility. As expected, a layer of ZRP primer is visible from the previous coating application underneath the NPE-ZRP repair layer. Some oxide 
formation is also evident on the old primer ZRP surface due to humidity pre-exposure, apparently a reason for additional barrier protection which is observed by the comparative higher impedance value. Consistent with all other exposure conditions salt contaminated samples showed more degradation in comparison to humidity exposed samples. Whereas for all other exposure conditions of NPE-ZRP coating, zinc consumption was observed from the bulk primer layer here for the hand cleaned samples the dominant consumption modality continues along with the primer/steel interface where old ZRP layer remain. Zinc oxidation at the steel/primer interface is apparent as the reason for the degradation of adhesion strength of the hand cleaned NPE-ZRP samples along with the lack of mechanical interlocking of the primer with the steel substrate.
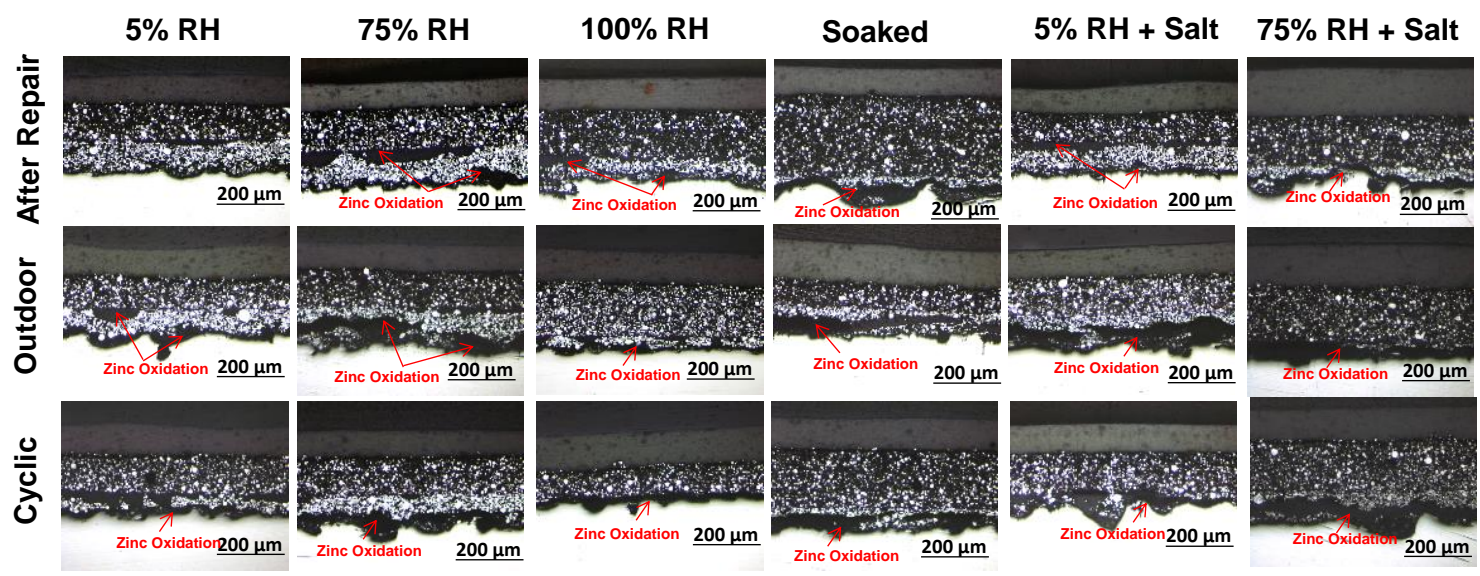

Figure 5.24. Micrograph of Handcleaned Unscribed NPE-ZRP Samples. (Pre-exposure conditions are noted for each coupon shown)

Figure $5.25 \& 5.26$ shows the cross-sectional micrograph of representative sandblasted and hand cleaned scribed NPE-ZRP samples after exposure along with the different prerepair condition. Each pre-exposure condition (before the repair coating application) is compared. Iron oxidation and zinc consumption were observed for all the samples regardless of the pre-exposure and surface cleaning differentiation. Indeed, OCP 
condition and formation of another capacitive loop indicates steel corrosion along with zinc consumption. Steel corrosion was predominant at the defect site whereas zinc consumption was either from the bulk primer layer (sandblasted samples) or at the primer/steel interface. Salt contaminated samples show greater degradation as osmotic blister formation renders the zinc pigments to provide cathodic polarization due to the discontinuity with the steel substrate. Table 5.1 summarize the information that quantifies the extent of degradation around the coating defect.

Table 5.1. Zinc Consumption Characterization of NPE-ZRP Scribed Samples.

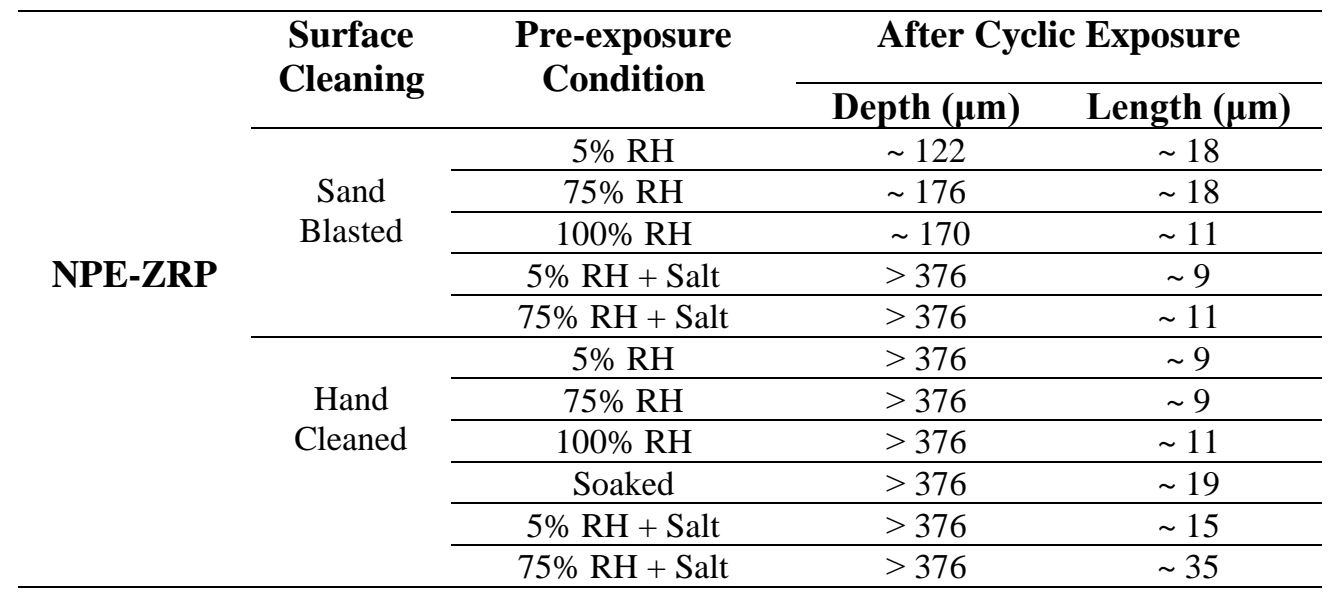



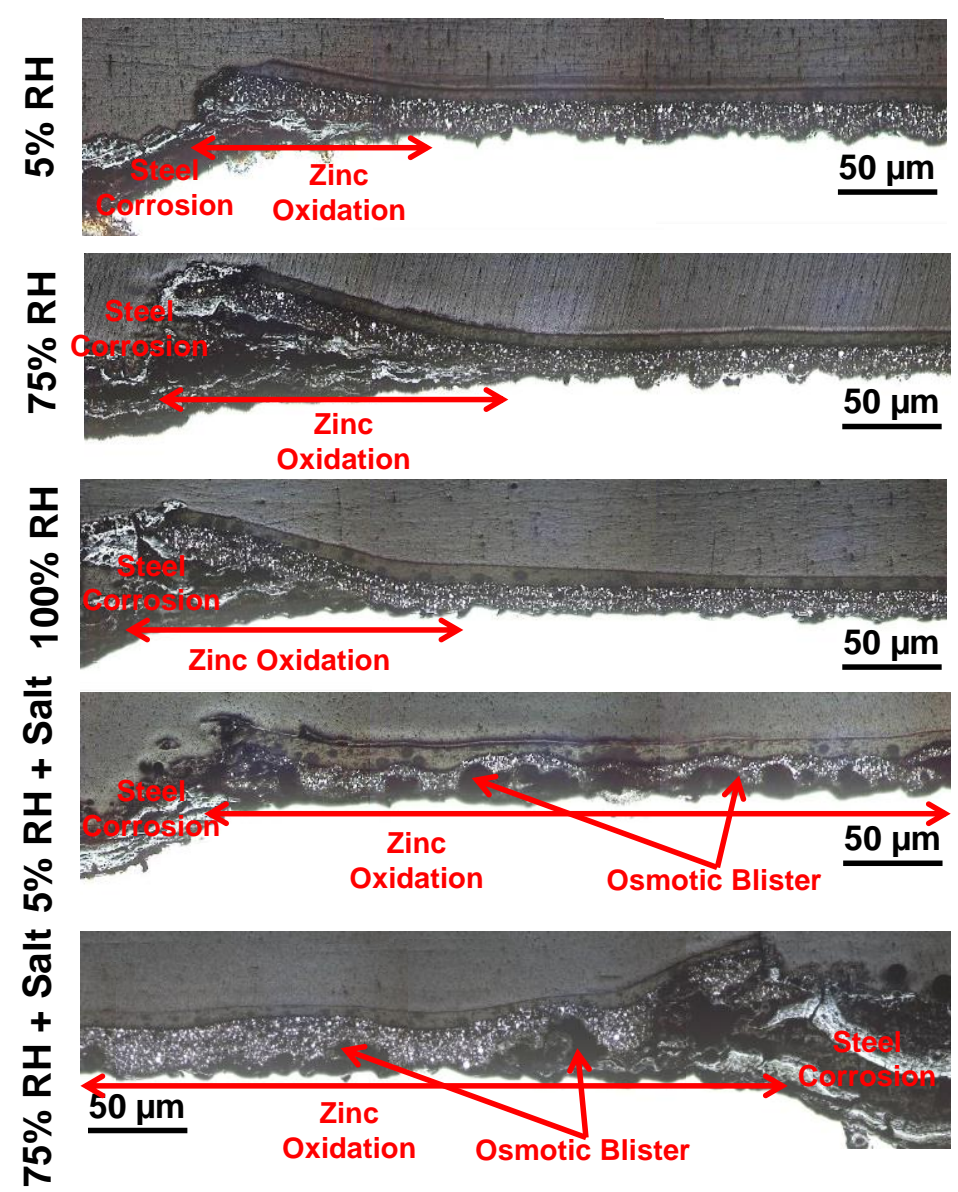

Figure 5.25. Micrograph of Sandblasted Scribed NPE-ZRP Samples.

(Pre-exposure conditions are noted for each coupon shown)

The consumption length clearly shows that the dominant zinc consumption for the hand cleaned NPE-ZRP repaired samples was along with the steel/primer interface. Regardless of the pre-exposure condition and surface contamination, with some steel corrosion around the introduced steel defect dominant zinc consumption continues along with the steel/primer interface. This differentiation occurred due to the remnant ZRP layer from the previous coating system where dominant consumption followed at the interface and with the formation of the zinc oxide layer acts as a barrier for the conductivity to the above NPE-ZRP layer. For the formation of the barrier layer, the 
influence of nano-particles on the mechanical bonding of the top NPE-ZRP layer was not persistent. Sandblasted NPE-ZRP coating also shows consumption along with the interface whereas there was early steel corrosion from insufficient galvanic protection especially around the defect site. Despite some level of degradation along with the interface around the defect site dominant zinc consumption occurred from the bulk primer layer. But the salt contaminated samples showed continuous degradation along with the interface due to the formation of osmotic blister which renders the connectivity of the zinc pigments with the steel substrate hence ineffective cathodic polarization.

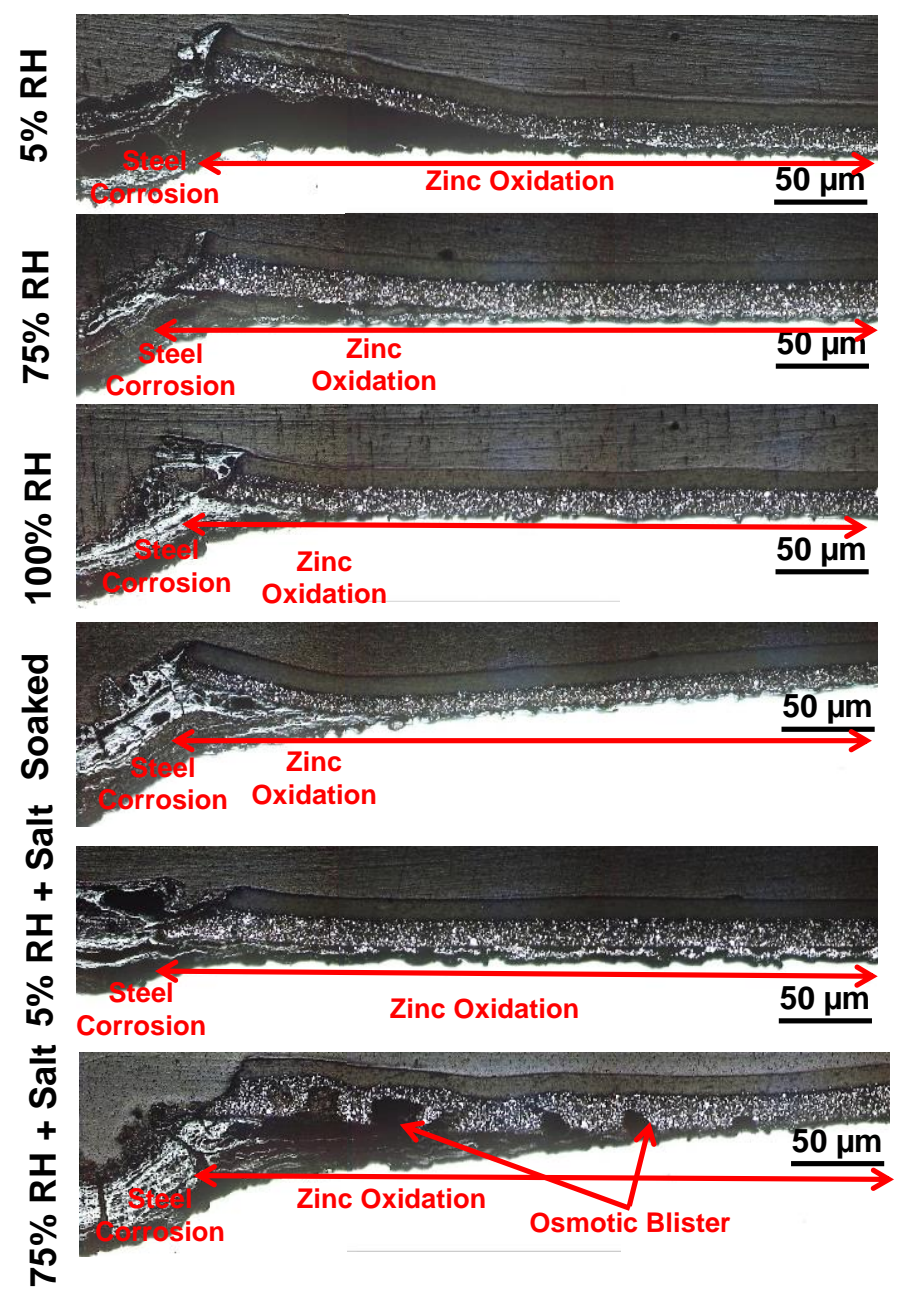

Figure 5.26. Micrograph of Hand Cleaned Scribed NPE-ZRP Samples. (Pre-exposure conditions are noted for each coupon shown) 


\subsection{ZRP Coating}

\subsubsection{Material Characterization}

A conventional three-coat system with an inorganic zinc-rich primer (ZRP) was tested in part to compare the performance of NPE-ZRP applied as a conventional repainting system. Hand cleaning was not incorporated in the test condition as sandblasting is specified for the proper application of inorganic zinc-rich coating systems. Steel coupons were exposed to different levels of moisture exposure and salt contamination after sandblast cleaning and prior to the application of conventional ZRP coating. Representative test coupons of repaired ZRP coating are shown in Figure 5.27. There were no outward visual indicators of coating degradation after the application of the three-coat system on the adverse pre-conditioned steel substrate. The measured coating thickness after coating application is presented in Figure 5.28.

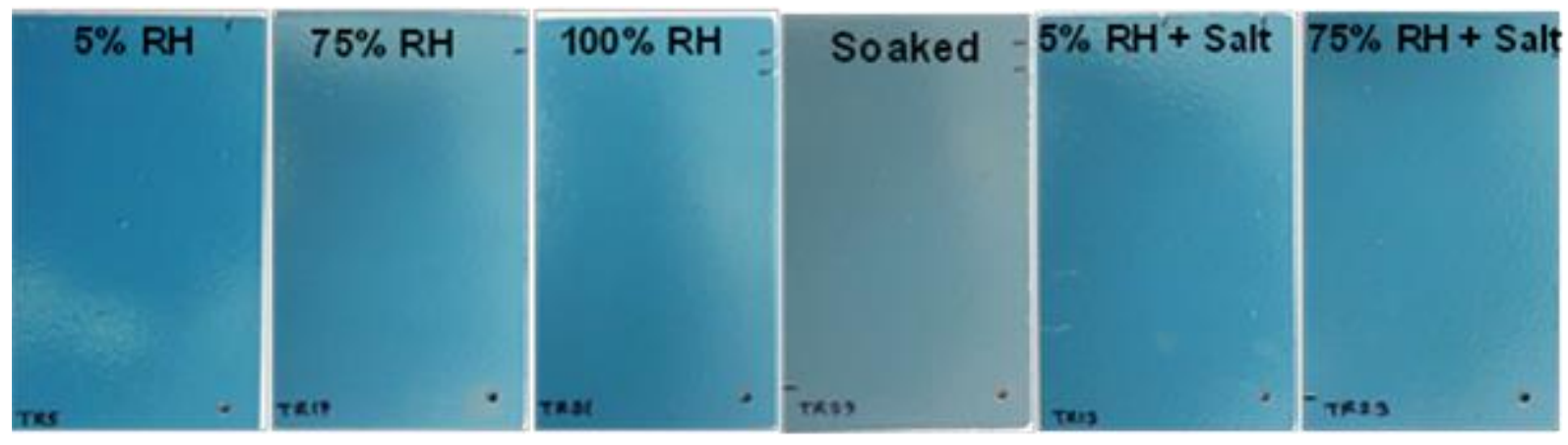

Figure 5.27. Repaired Coupons after Application of ZRP Coating.

(Pre-exposure conditions are noted for each coupon shown)

There was general variability in sample thickness due to the inconsistent application of the three coating layers (median thickness of the coupons was between $\sim 275 \mu \mathrm{m}$ and $\sim 400 \mu \mathrm{m})$. Apart from application variability, there was an indication of greater coating thickness for samples pre-exposed to moisture and salt contamination 
(apparently due to the addition of extra oxide layer) compared to the relatively more benign 5\% RH pre-exposure case. Cross-sectional micrographs of representative samples (Figure 5.29) showed that presence surface rusting occurred on the samples after sandblasting and prior to coating application according to the variability of pre-exposure. This surface oxidation was greatest for the samples contaminated with salt prior to coating, consistent with the high coating thickness measured there.

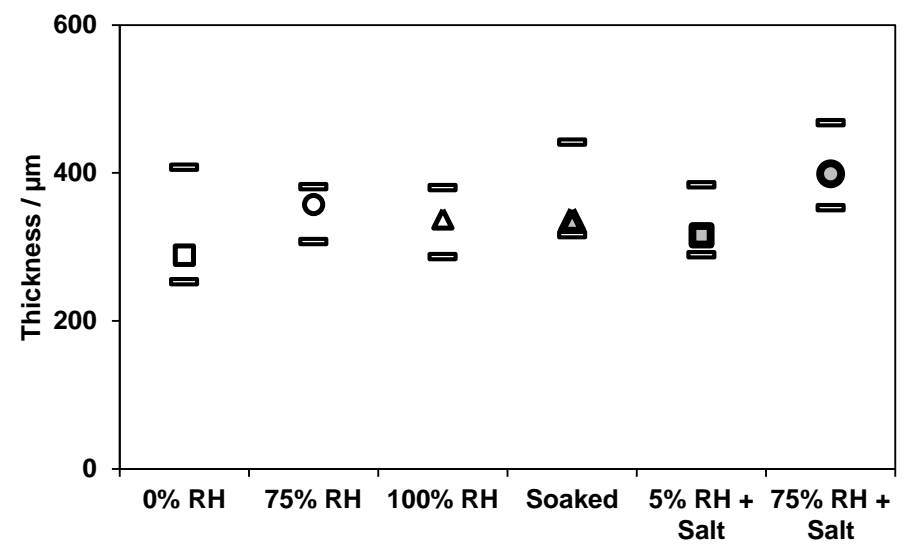

Figure 5.28. Coating Thickness of ZRP Coupons after Repair.
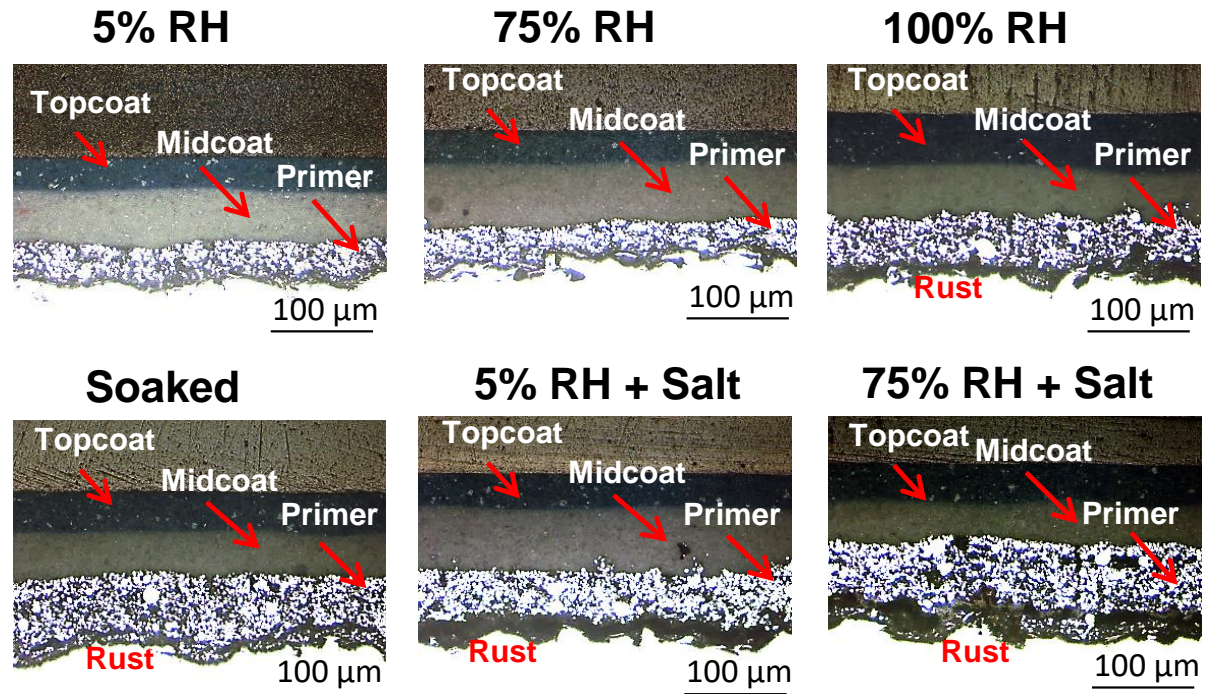

Figure 5.29. Optical Micrograph of ZRP Coating Repair Coupon. 
The coating pull-off strengths of the repaired coupons are shown in Figure 5.30. Pull-off results were categorized as a topcoat adhesive failure when the dolly had remnants of the topcoat material and the coupon retained the off-white-colored midcoat. The failure was considered a primer cohesive failure when both the dolly and the coupon retained the primer. The failure was considered a primer adhesive failure when the dolly had the silver-colored primer and the coupon showed some level of grey that could be from the steel substrate, remnant mill scale, or initial surface rust. The pull-off strength testing generally resulted in the failure of coating components, with strengths below the minimum FDOT requirements (800 psi, $5516 \mathrm{kPa}$ ) for all test conditions. The pull-off strength testing resulted in lower pull-off strengths for the samples exposed to $75 \% \mathrm{RH}$ and samples contaminated with salt, indicating the negative effect of the improper surface preparation. In the relatively benign $5 \%$ and $75 \%$ pre-exposure cases, the pull-off strength testing resulted in topcoat adhesive failure. In higher moisture exposure and with salt contamination, the testing resulted in failures of the coating primer. Representative pictures of different failure modalities are shown in Figure 5.31.

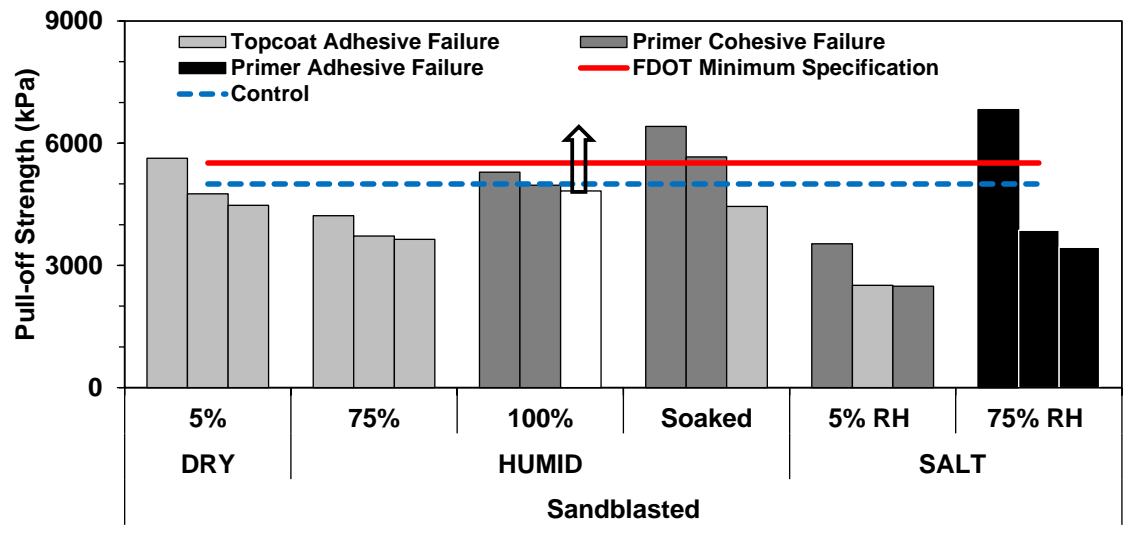

Figure 5.30. Coating Pull-off Strength of ZRP Coupons after Repair. 

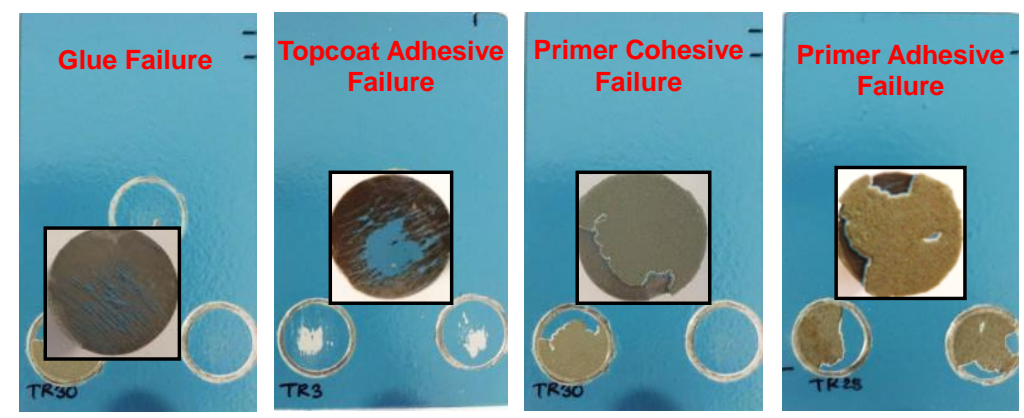

Figure 5.31. Failure Modalities of Pull-off Strength Testing for ZRP Coupons.

\subsubsection{Results and Discussion}

\subsubsection{Outdoor Exposure}

\subsection{Visual Observation}

Representative pictures of the ZRP coupons after outdoor exposure are shown in Figure 5.32 (Detail in Appendix C). No significant coating deterioration was observed up to 8 months of outdoor exposure, but some level of discoloration was observed apparently due to photochemical reaction by the environmental attack (light, heat, moisture, and oxygen) on the exposed coated surface. Surface discoloration started within 1 month of exposure and progressively deteriorated over the 8 months of the exposure period. 


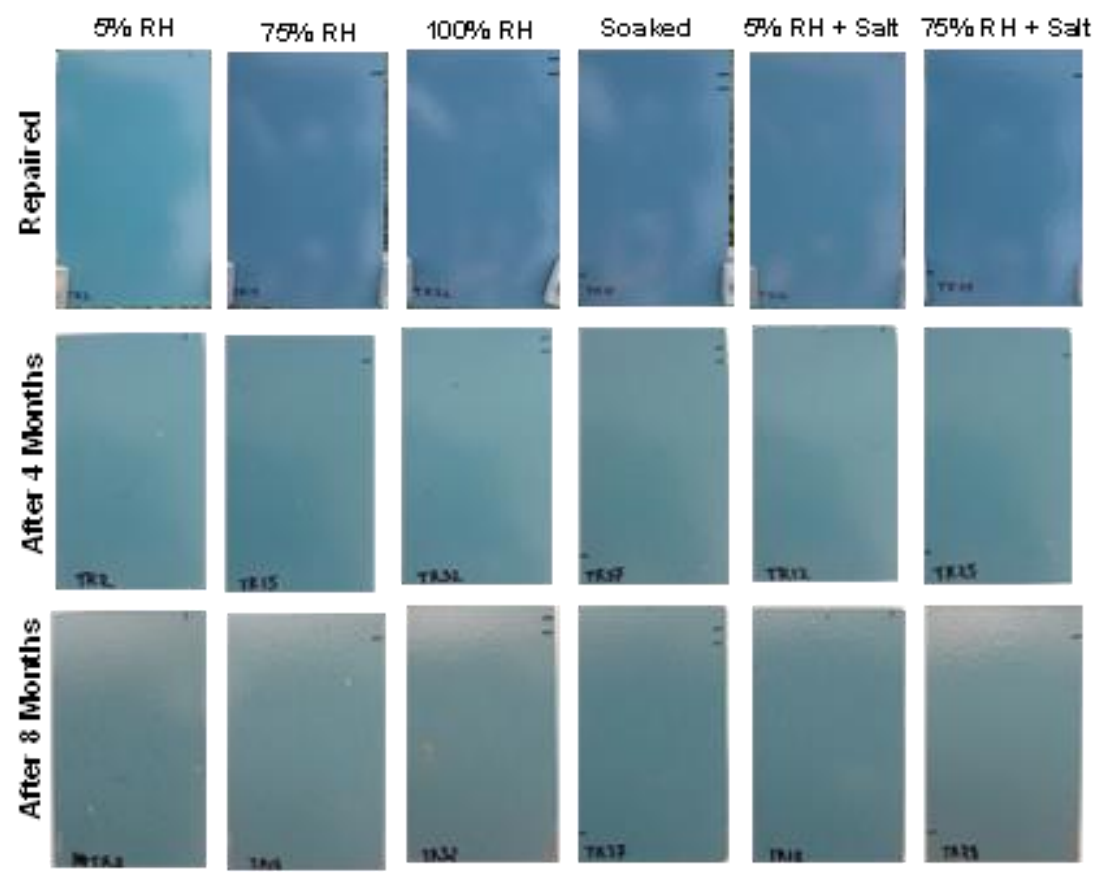

Figure 5.32. Condition of the Sandblasted ZRP Samples after Outdoor Exposure.

\subsection{Coating Thickness}

As shown in Figure 5.33, no significant changes in coating thickness were measured during the 8 months of exposure. The coating thickness measurements could not readily resolve any extent of coating degradation. The apparent differentiation of coating thickness due to the development of surface oxide layer during the pre-exposure, generally observed for the entire test sample population, was not well-manifested for the subpopulation of samples exposed in outdoor exposures. A slight decrease in coating thickness apparently due to the polymer degradation by the atmospheric attack at the outdoor environment whereas the increment is evidently associated with the corrosion product developed with further exposure. 


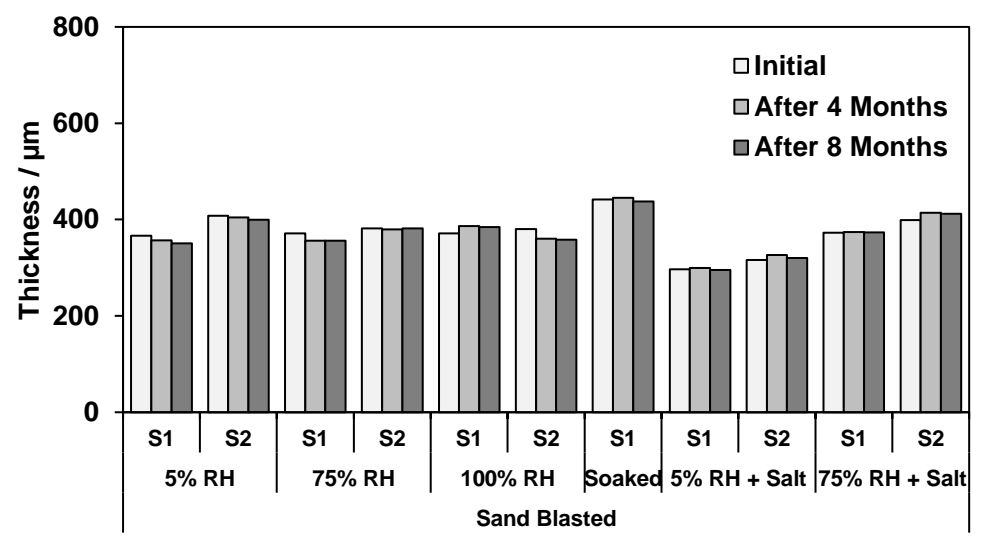

Figure 5.33. Coating Thickness Change of ZRP Samples after Outdoor Exposure.

\subsection{Pull-off Strength Test}

After exposure, all samples exhibited degradation in coating adhesion and loss in pull-off strength (relative to the testing immediately after coating application) as shown in Figure 5.34. Furthermore, all testing resulted in lower pull-off strengths than control ZRP samples indicating the detrimental effect of the improper surface preparation along with outdoor exposure. Like earlier testing after repair coating application, ZRP samples failed to meet minimum strength for all cases. In comparison to the coating pull-off strengths for samples immediately after coating application, testing resulted in failure at the primer for all test conditions, including the relatively benign $5 \%$ and $75 \%$ RH preexposure (where separation previously occurred at the topcoat). Primer adhesive failure occurred at values less than $\sim 3,000 \mathrm{kPa}$ for the salt-contaminated samples. The detail of failure modality is shown in Appendix D. 


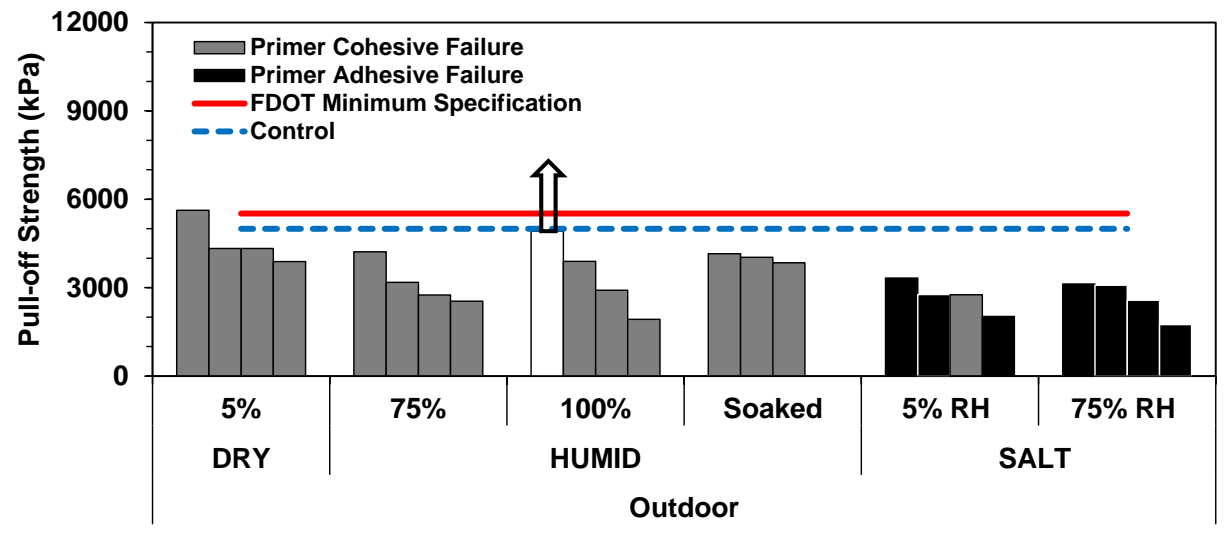

Figure 5.34. Pull-off Strength of ZRP Coupons after Outdoor Exposure.

\subsubsection{Cyclic Exposure}

\subsection{Visual Observation}

Photo documentation of ZRP samples in the as-received (pre-exposed) condition and after each exposure cycle was made. Representative photos of coated samples in the as-received condition and after $3^{\text {rd }}$ and $20^{\text {th }}$ cycle (5 months) of exposure with and without intentional coating defects are presented in Figures 5.35-5.36 (Detail in Appendix C), respectively. No major indication of coating deterioration was observed for the ZRP unscribed samples throughout the cyclic wet/dry exposure, but minor surface roughening and discoloration was observed. The coating appeared intact throughout the test exposure. For samples with an introduced coating defect, rust bleed-out was observed within the defect site. The rust was observed after the salt solution immersion step in the first cycle. The extent of outward bleed-out appeared to increase and continue throughout the remainder of the test cycles. Accumulation of corrosion products at the defect site was not observed over time due to the change of test electrolyte for each wet cycle and the vertical orientation during the salt-fog exposure. 


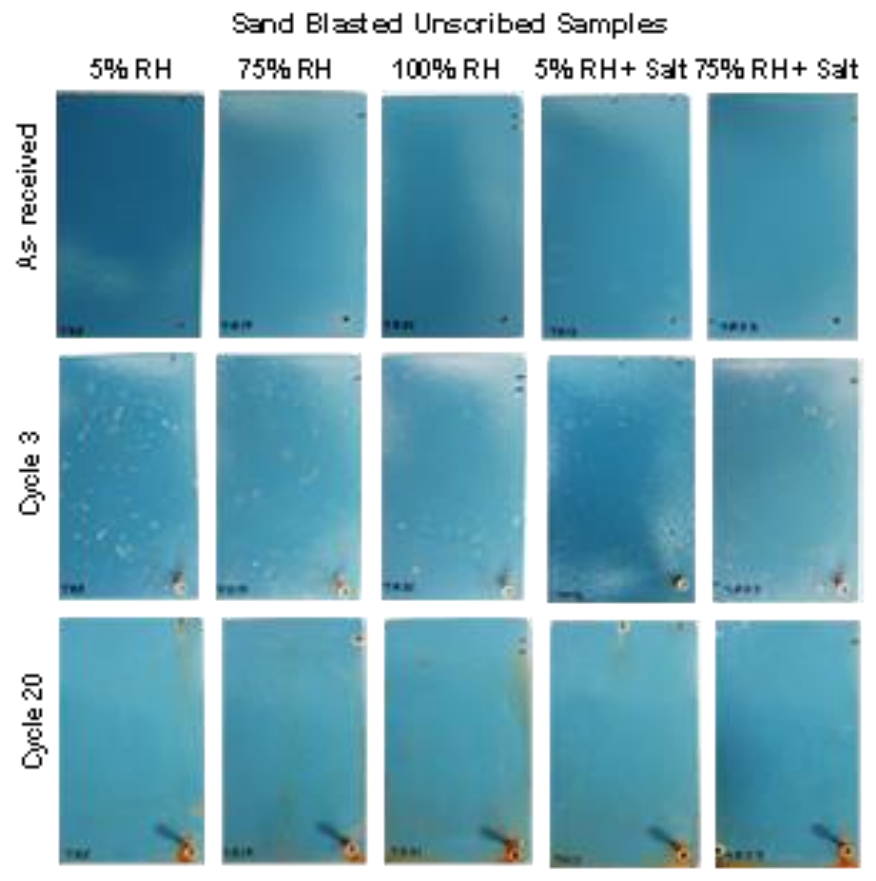

Figure 5.35. Typical Condition of Scribed ZRP Samples after Cyclic Exposure.

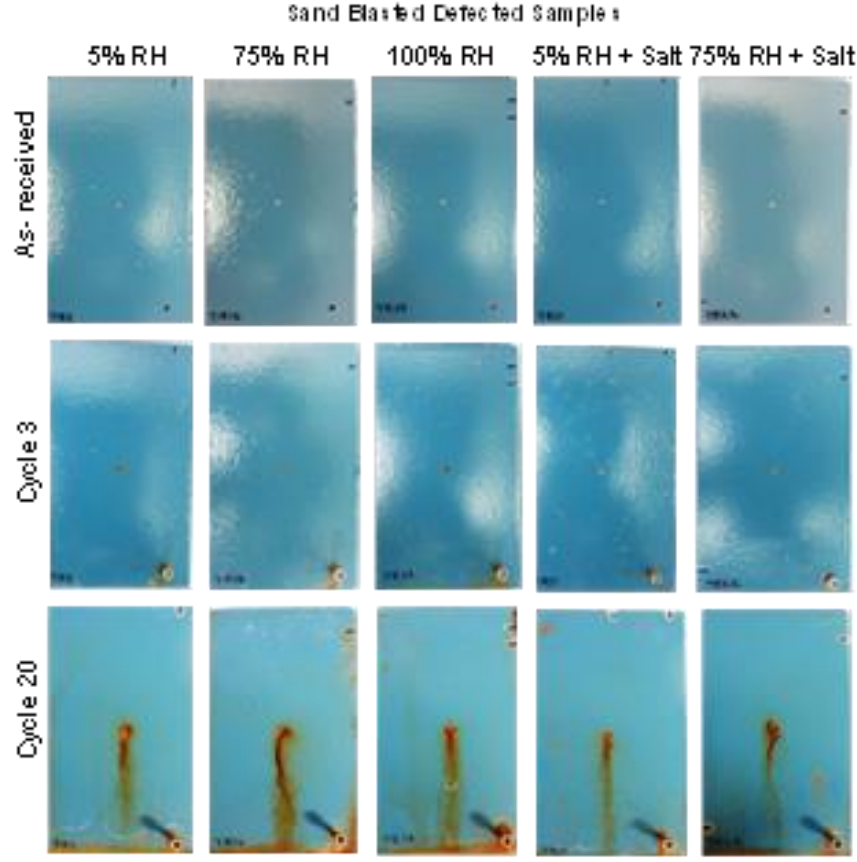

Figure 5.36. Typical Condition of Unscribed ZRP Samples after Cyclic Exposure.

\subsection{Coating Thickness}

Coating thickness was measured at each third cycle of exposure after the dry 
phase. Figures 5.37-5.38 represents the measured coating thicknesses with exposure for the scribed and unscribed samples. Both unscribed and scribed samples showed similar behavior, apparently no effect of coating defect in coating thickness. Consistent with the outdoor exposure results, thickness change did not show a clear effect of the various levels of pre-exposure to moisture and salt contamination (prior to coating application). As the samples were subjected to the cyclic testing, indicating that the steel surface oxide layer that formed prior to coating did not significantly change with the introduction of moisture and salt during the cyclic testing as well as coating thickness. Variation in the initial coating thickness was apparently due to the application inconsistency as well as developed rusting during pre-exposure. Apparent increment in the thickness observed due to the oxidation of zinc pigments with the activation by the ingression of electrolyte or oxide formation due to the corrosion of steel substrate whereas decrease can be associated with the polymer degradation due to hydrolysis. Thickness increase and decrease apparently counteract each other and didn't reveal any clear indication of degradation during the course of exposure.
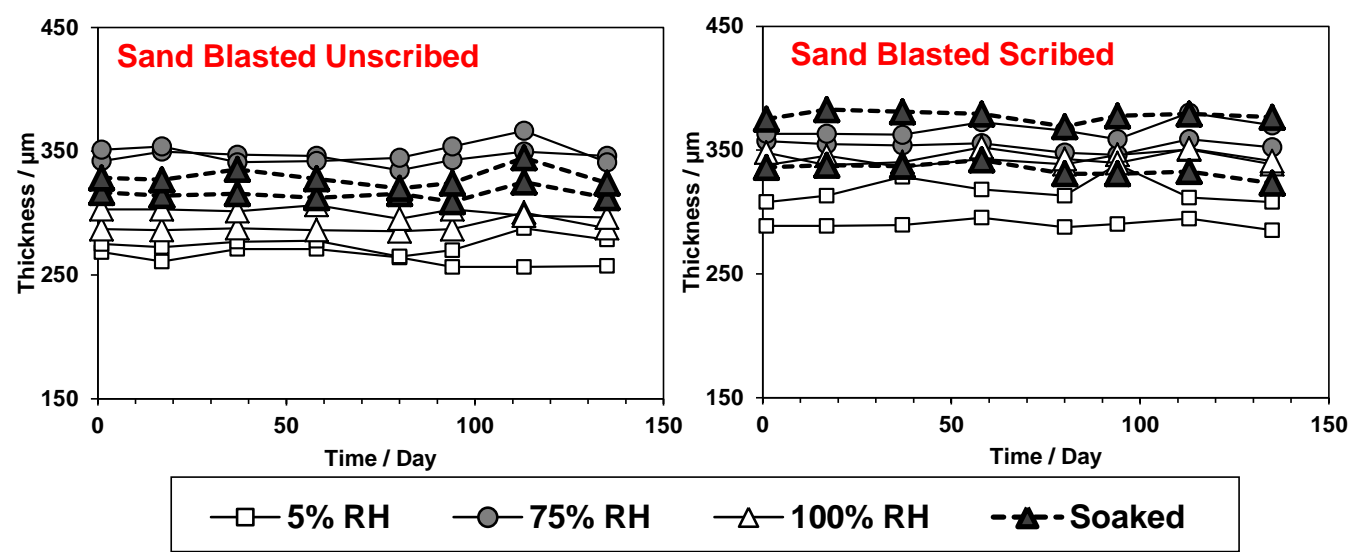

Figure 5.37. Coating Thickness of ZRP Coupons after Cyclic Exposure. (Moisture Exposure during Coating Application) 


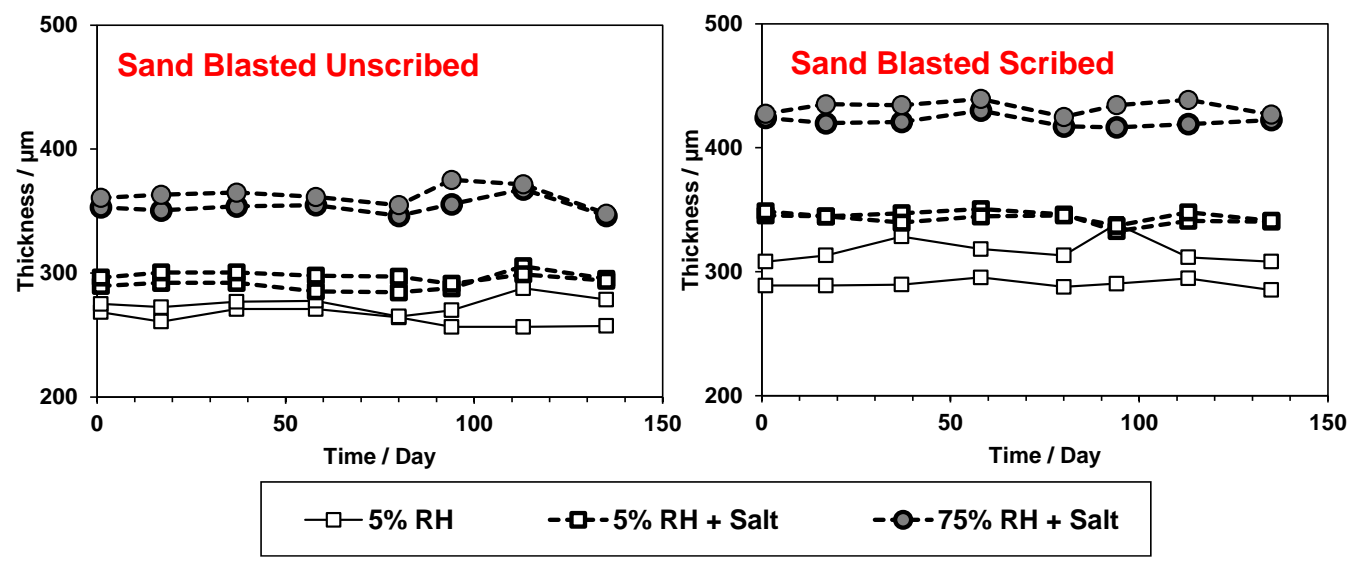

Figure 5.38. Coating Thickness of ZRP Coupons after Cyclic Exposure. (Salt Exposure during Coating Application).

\subsection{Pull-off Strength Test}

After cyclic exposure, all samples exhibited similar degradation as described for samples immediately after coating application and after outdoor exposure (Figure 5.39). Like the samples for the other exposure conditions, the ZRP samples failed to meet minimum strength for all cases, and all testing resulted in lower pull-off strengths than control ZRP samples, further implicating the negative effect of the improper surface preparation. The pull-off test failure modality generally resulted in primer failure except for the $5 \% \mathrm{RH}$ pre-exposure case. Pre-exposure to humidity and salt contamination, like for the other exposure conditions, resulted in primer adhesive failure at relatively low pull-off strengths $(<3,000 \mathrm{kPa})$. The detail of failure modality is shown in Appendix D. 


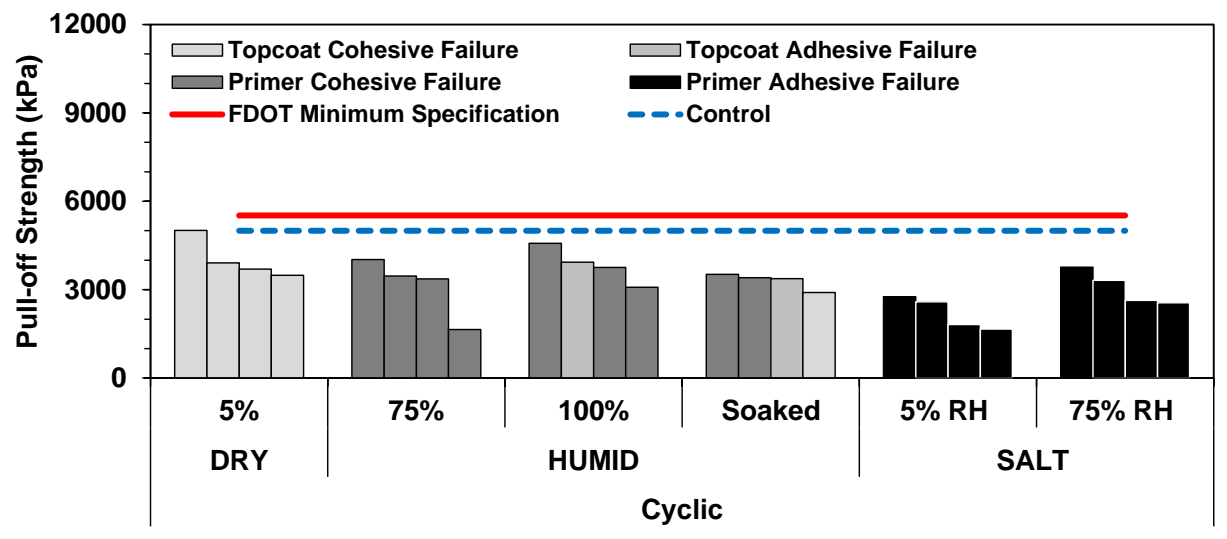

Figure 5.39. Pull-off Strength of ZRP Coupons after Cyclic Exposure.

\subsubsection{Electrochemical Analysis (OCP-LPR)}

OCP and $\mathrm{I}_{\text {corr }}$ result for unscribed and scribed ZRP samples with the various precoating environmental conditioning are presented in Figures 5.40-5.41. For unscribed samples, the relatively noble potential values obtained in initial OCP measurements indicated good coating barrier characteristics early in the exposure regiment. Around day 10 , the potentials dropped to negative values $\left(\sim-0.8 \mathrm{~V}_{\mathrm{SCE}}\right)$ indicative of moisture activating the zinc pigments but rebounded to noble potentials $\left(-0.2 \mathrm{~V}_{\mathrm{SCE}}\right)$ until the end of the test exposure at day 140. The corrosion currents for the unscribed samples, consistent with the OCP trends, were very low (in the order of $10^{-5} \mu \mathrm{A}$ ) for all cases. The development of surface rust prior to coating application would likely be a factor in the corrosion development possibly where moisture availability could be enhanced.

However, this was not manifested for the other samples that had surface oxidation prior to the coating application. Also, the coating degradation described by loss of coating adhesion was observed for all the pre-exposure conditions and was not well-correlated to the developed corrosion currents.

For the scribed samples, early negative potentials $\left(\sim-1.0 \mathrm{~V}_{\mathrm{SCE}}\right)$ were indicative of 
exposed zinc activity at the periphery of the coating defect. The shift to more noble potentials was indicative of zinc consumption. This potential shift may suggest less effective cathodic protection. The higher corrosion currents were consistent with the larger exposed steel area than compared to the non-scribed samples. The initially high corrosion rates were likely due to the early zinc activity, but after depletion of the zinc, the remnant zinc was not readily accessible to afford continued beneficial cathodic polarization, as exhibited by the continued steel corrosion that developed at the defect site and the subsequent increase in steel corrosion current. The effect of the precoating moisture exposure and salt contamination was not well-manifested in the electrochemical characteristics of the samples. For comparison, the terminal potential and $\mathrm{I}_{\mathrm{corr}}$ of scribed samples from the testing of control samples (described in Chapter 5) are presented here as doted red (30-day immersion) and the blue line (60-day immersion). Similar behavior was observed for control tests, indicating the general electrochemical behavior of zincrich primers exposed to the solution. 

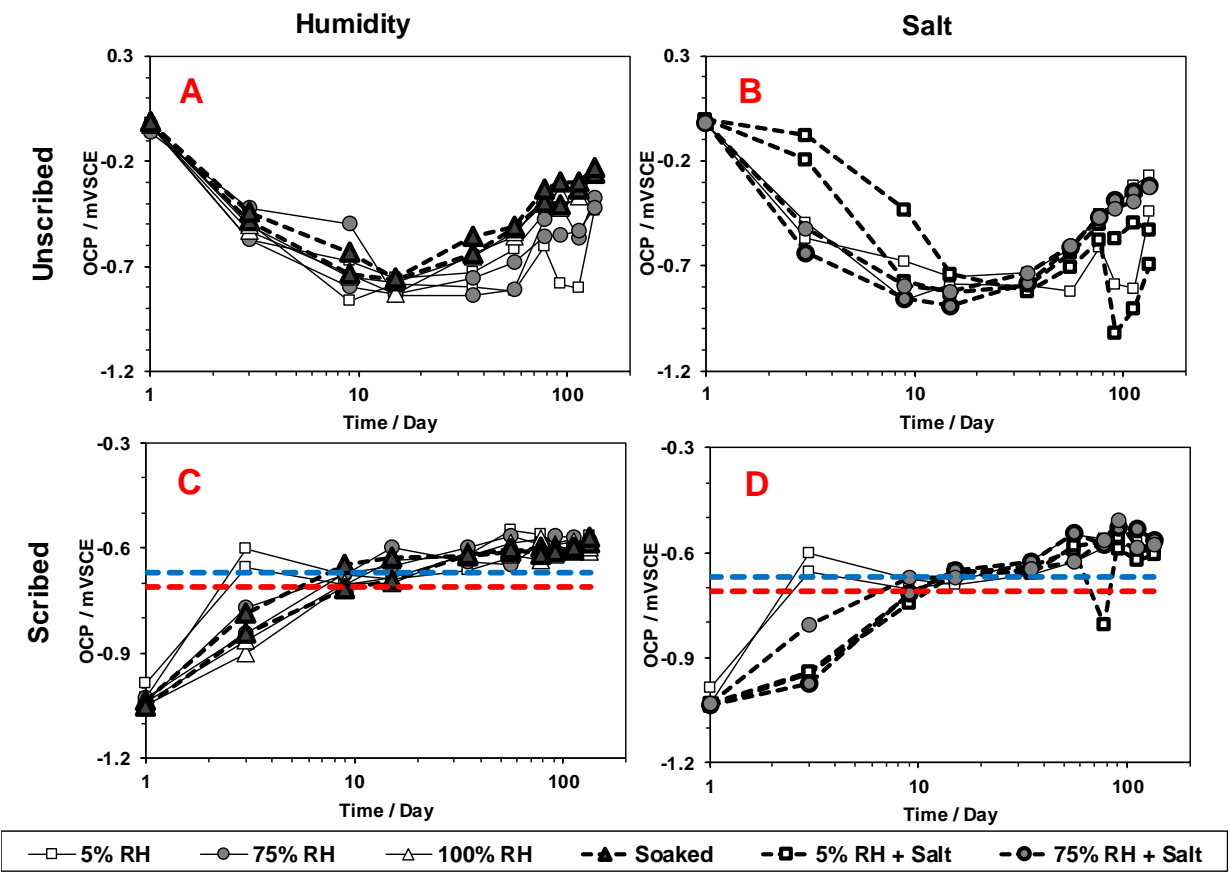

Figure 5.40. OCP of ZRP Samples during Cyclic Exposure.

A) Moisture Exposure of Unscribed Samples. B) Salt Exposure of Unscribed Samples. C) Moisture Exposure of Scribed Samples. D) Salt Exposure of Scribed Samples.
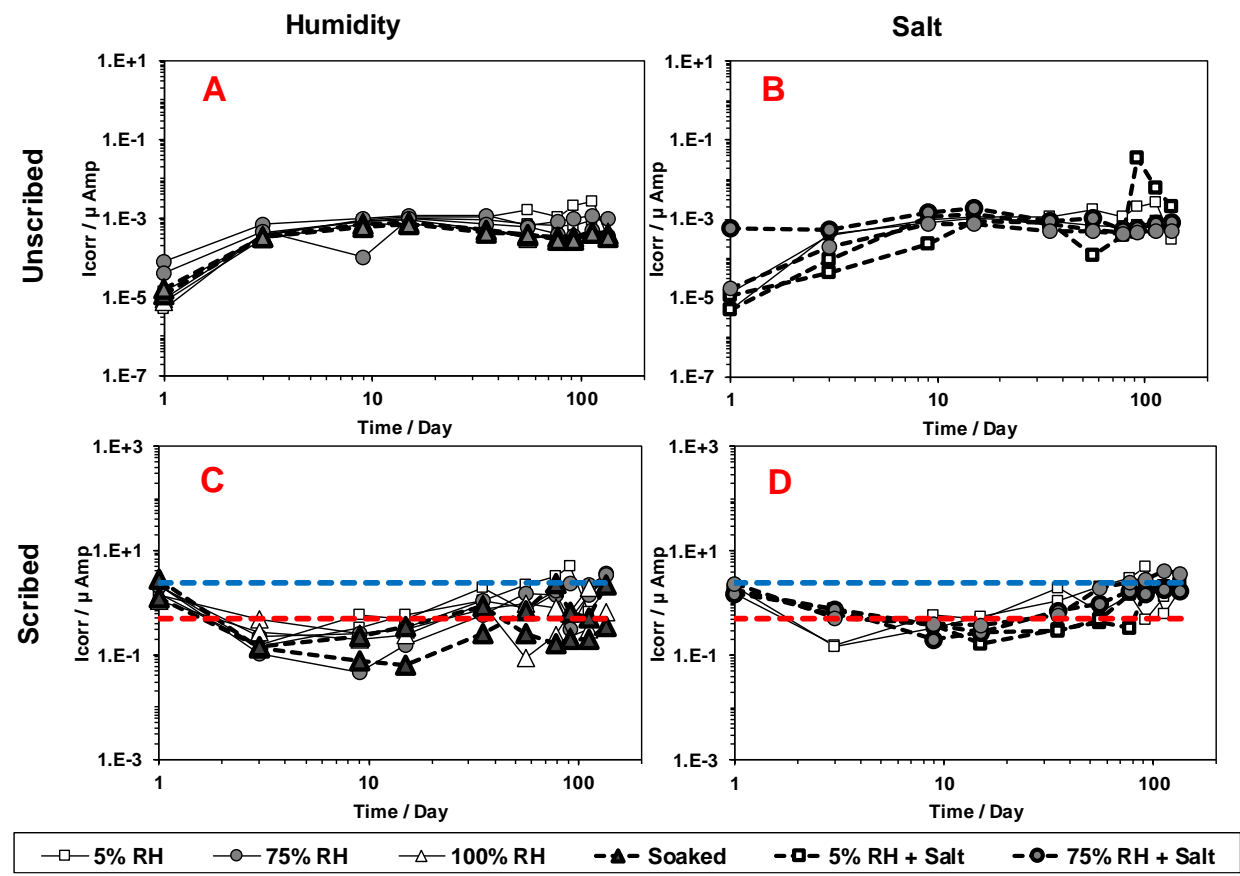

Figure 5.41. Icorr of ZRP Samples During Cyclic Exposure.

A) Moisture Exposure of Unscribed Samples. B) Salt Exposure of Unscribed Samples. C) Moisture Exposure of Scribed Samples. D) Salt Exposure of Scribed Samples. 


\subsubsection{Characterization of Zinc Consumption}

Cross-sectional micrographs were analyzed to quantify zinc consumption. Figure 5.42 shows the cross-sectional micrograph of representative sandblasted unscribed ZRP samples after the end of outdoor (8 months) and cyclic (5 months) exposure in consideration to the after-repair condition. Each pre-exposure condition (before the repair coating application) is compared to identify different exposure susceptibility. Samples with different pre-exposure conditions showed similar behavior in both outdoor and cyclic exposure. Consistent with the behavior of ZRP samples for the application of new structural steel (described in chapter 5) zinc consumption was observed along with the steel/ primer interface. However, as observed in Figure 5.42, no difference detected in the modality of zinc degradation based on the pre-exposure condition. Despite the preexposure condition, the zinc consumption continues along with the steel/ primer interface. This behavior is also coherent with the electrochemical results (OCP and EIS) of a potential shift to more active value and again back to noble potential which is apparently due to the activation of zinc pigments for the diffusion of electrolyte and eventual formation of zinc oxide which is evidently displays the barrier protection with high impedance value. However, for the salt contaminated samples, the degradation was greater due to the presence of salt concentration which accelerates the reaction rate. Degradation of the bulk primer layer was also observed in forms of crack formation apparently due to the improper bond with the steel substrate as the coating was applied over the oxidized steel substrate. The degradation at the coating interface is consistent with the lower pull-off strength results and also consistent for the salt contaminated 
samples where the adhesive failure was observed at the interface as well as the significant drop of the capacitive loop of the Nyquist diagram with exposure.

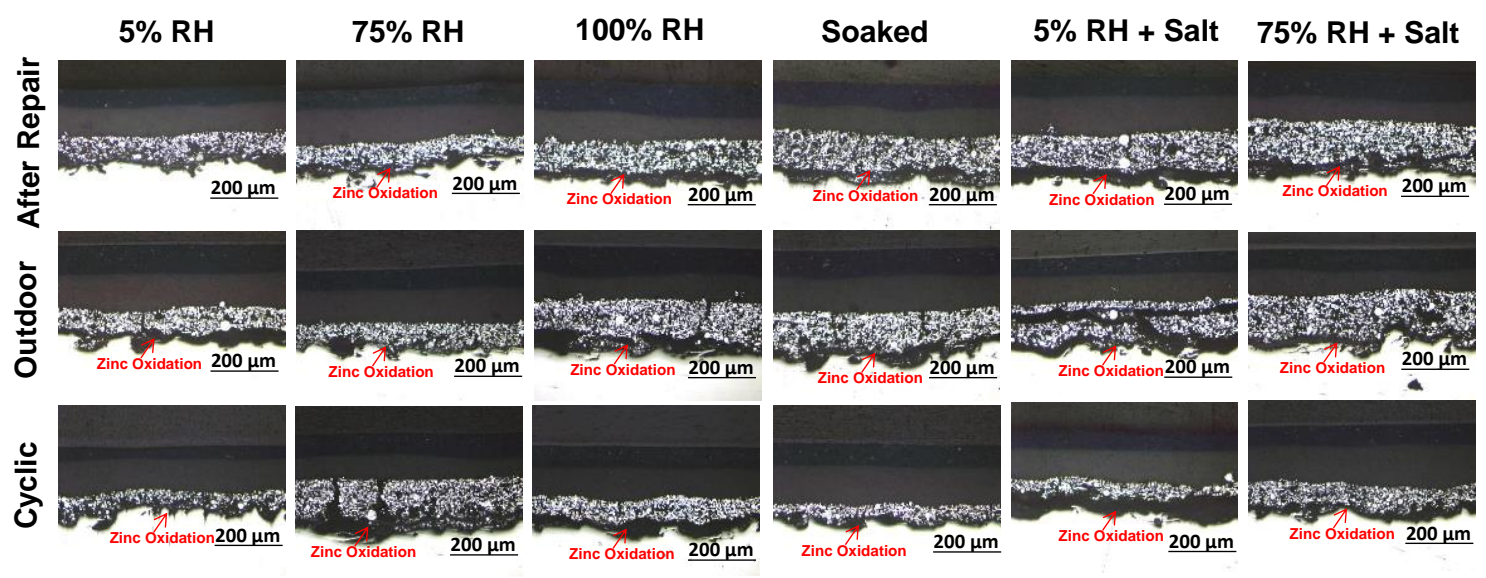

Figure 5.42. Micrograph of Hand Cleaned Unscribed ZRP Samples.

(Pre-exposure conditions are noted for each coupon shown)

An image processing software was used to quantify the amount and modality of zinc consumption in consideration to the as-received zinc content by calculating the reduction of actual zinc area. Figure 5.43 shows the level of zinc consumption for the unscribed samples in terms of percent activity. A similar trend of zinc consumption was observed at different exposure environment along with the different pre-exposure condition. Zinc consumption percentage after environmental exposure did not show a significant increase apparently portrays the good barrier effect of the three-coat system. Consistent with all other findings, salt contaminated samples with 5\% RH pre-exposure condition degraded more with the exposure apparently due to the more absorption of moisture to the salt crystals and further reaction. 


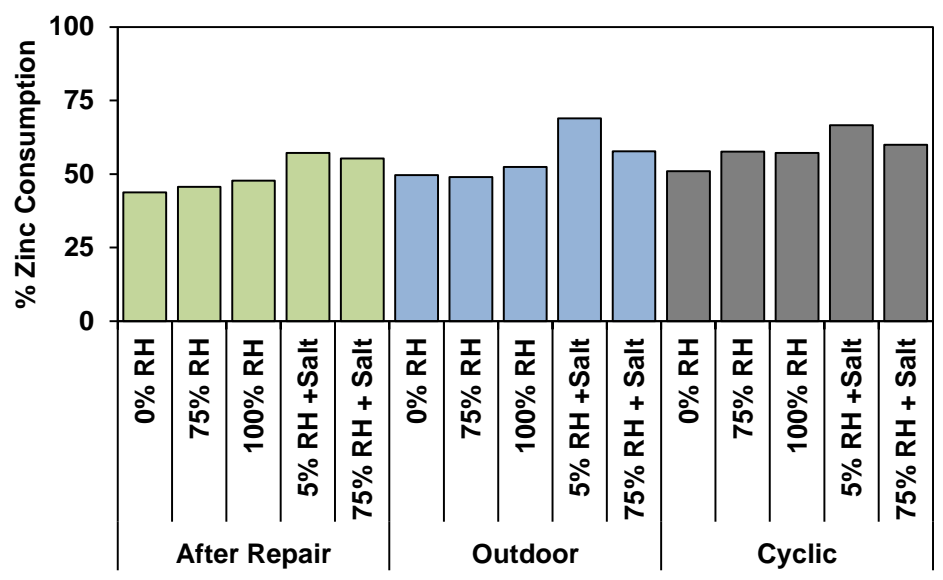

Figure 5.43. Zinc Consumption of Sandblasted Unscribed ZRP samples.

Figure 5.44 shows the cross-sectional micrograph of representative scribed ZRP samples after exposure along with the different pre-repair condition. Each pre-exposure condition (before the repair coating application) is compared. Iron oxidation and zinc consumption were observed for all the samples regardless of the pre-exposure and surface cleaning differentiation. Indeed, OCP condition and formation of another capacitive loop indicates steel corrosion along with zinc consumption. Steel corrosion was predominant at the defect site whereas zinc consumption was at the primer/steel interface. Salt contaminated samples show greater degradation as oxide formation (from the reaction with salt) renders the zinc pigments to provide cathodic polarization due to the discontinuity with the steel substrate. Table 5.2 summarizes the information that quantifies the extent of degradation around the coating defect. 

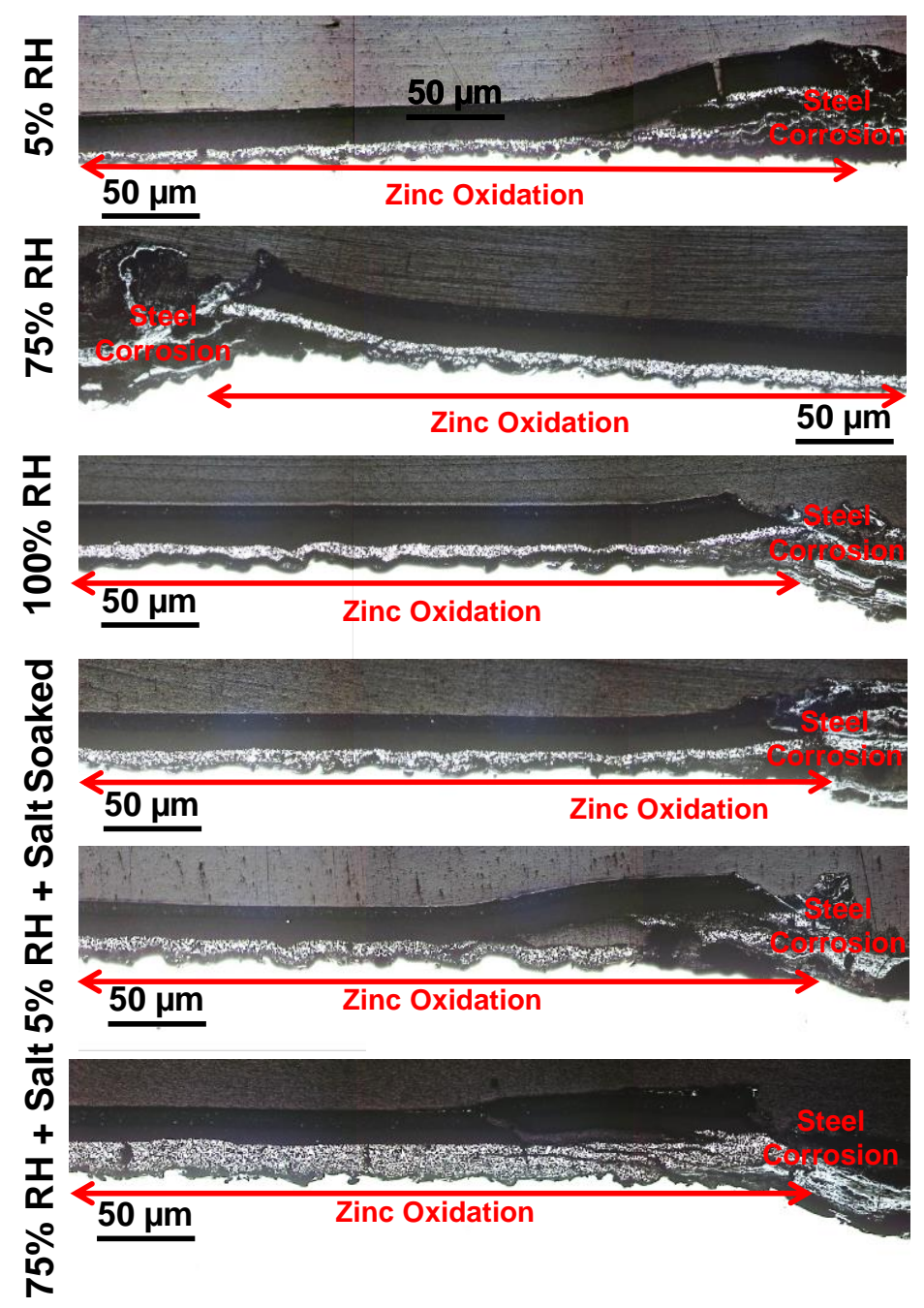

Figure 5.44. Micrograph of Sandblasted Scribed ZRP Samples.

(Pre-exposure conditions are noted for each coupon shown)

Table 5.2. Zinc Consumption Characterization of ZRP Scribed Samples.

\begin{tabular}{|c|c|c|c|c|}
\hline \multirow{8}{*}{ ZRP } & \multirow{2}{*}{$\begin{array}{l}\text { Surface } \\
\text { Cleaning }\end{array}$} & \multirow{2}{*}{$\begin{array}{l}\text { Pre-exposure } \\
\text { Condition }\end{array}$} & \multicolumn{2}{|c|}{ After Cyclic Exposure } \\
\hline & & & Depth $(\mu \mathrm{m})$ & Length $(\mu \mathrm{m})$ \\
\hline & \multirow{6}{*}{$\begin{array}{l}\text { Sand } \\
\text { Blasted }\end{array}$} & $5 \% \mathrm{RH}$ & $>376$ & $\sim 6$ \\
\hline & & $75 \% \mathrm{RH}$ & $>376$ & $\sim 6$ \\
\hline & & $100 \% \mathrm{RH}$ & $>376$ & $\sim 6$ \\
\hline & & Soaked & $>376$ & $\sim 6$ \\
\hline & & $5 \% \mathrm{RH}+$ Salt & $>376$ & $\sim 9$ \\
\hline & & $75 \%$ RH + Salt & $>376$ & $\sim 9$ \\
\hline
\end{tabular}


The consumption length clearly shows that the dominant zinc consumption was along with the steel/primer interface. Regardless of the pre-exposure condition and surface contamination, with some steel corrosion around the introduced steel defect dominant zinc consumption continues along with the steel/primer interface. But the salt contaminated samples showed greater degradation along with the interface due to the formation of the oxide layer which renders the connectivity of the zinc pigments with the steel substrate hence ineffective cathodic polarization. 


\section{CHAPTER 6}

\section{IMPROVEMENTS IN COATING DURABILITY BY NANO-PARTICLES}

\subsection{Background}

Zinc-rich coating systems can be organic or inorganic in nature based on binder characteristics. Inorganic zinc-rich primers are a composite mixture of metallic zinc dust and a reactive binder solution usually a silicate, sodium silicate, potassium, lithium and ethyl silicate (mostly used nowadays). The individual zinc particles are surrounded and interlocked chemically by the inert binder matrix. With the loss of solvent (either water or alcohol) the reactive binder gets concentrated and causes some ionization of zinc and form silicic acid by the hydrolysis of silicate which forms a complex zinc silicate matrix that binds the metallic zinc pigments together [Charles C. Munger]. The silicic acid reacts with the ferrous ions on the steel substrate and creates an intimate chemical bonding with the surface, additional mechanical adhesion occurred due to wetting of the surface profile. In mechanical adhesion coating penetrates into the pores or voids of a rough surface, then interlocks onto the substrate and forms the bond. Thus, the silicate matrix gives both cohesive and adhesive strength to the cured primer matrix.

Organic zinc-rich primers contain metallic zinc particles encapsulated in a variety of resins, such as chlorinated hydrocarbons, epoxies (mostly used) and other polymers. Epoxy resins are mostly considered for ease of cure and processing, excellent moisture, solvent as well as chemical resistance and good adhesive strength. The organic resin forms a mechanical bond with the steel substrate by penetrating and interlocking into the pores or voids of a rough surface when a wet coating is applied. The mechanical interlocking theory describes adhesion based on the interaction between adhesive joining 
surfaces by high surface roughness and their mutual mechanical joining [S. J. Marshall, et. al., 2010]. According to the theory, adhesion occurs by the penetration of adhesives into pores, cavities, and other surface irregularities of the substrate surface by displacing the trapped air. Epoxy coatings are prone to the diffusion of corrosive agents (i.e., oxygen, water, and ions) with exposure to aggressive environments. The hydroxyl ions $\left(\mathrm{OH}^{-}\right)$generated at the cathode site increase the $\mathrm{pH}$ concentration underneath the coating (interface) facilitates the degradation of the adhesion of the coating with steel substrate and thus accelerates the corrosion of the metal beneath the coating.

Inorganic ZRP's are reported to provide longer corrosion protection in comparison to organic ZRP's. But zinc-rich epoxies provide some advantages compared to zinc silicates such as less demanding curing conditions (epoxies cure faster regardless of the RH level), easier to repair (less challenging surface cleaning and overcoating). Zinc silicates often develop mud cracking even at moderate film thickness which is very vulnerable for the bonding of primer to the steel substrate. Furthermore, another drawback is the topcoating the inorganic zinc-rich coatings because of the porous nature of the primer. The binder partially wets and binds the zinc particles together and to the substrate which often leads to the formation of pinholes, voids, or bubbles that facilitate the ingression of electrolytes as well as corrosion initiation. Good adhesion of the coating to the steel substrate can minimize corrosion and is the most important aspect of long-term durability. The mechanism for the bonding between the coating and the substrate interface has been a primordial critical topic for theoretical research of surface engineering. NPE-ZRP coatings expected to have improved mechanical performance due to the addition of nanoparticles. Usually, nano-particles are covalently bonded to the epoxy resin matrix. The 
mechanical performances of the composite coatings are strongly influenced by the phase morphology developed during mixing of nano-particles and the polymers, and the interfacial adhesion between the phases. The good interfacial adhesion between tubes and polymer matrix, uniform dispersion of nanotubes into the matrix and adhesion of the coating with the substrate material is required to enhance the mechanical performance.

The adhesion between the coating layer and the substrate surface can be affected by the surface roughness, substrate porosity, irregularities on the substrate, density of adhesives, environmental conditions during the coating application and wettability of the substrate. The compatibility of the coating components may be compromised when applied in non-ideal conditions. For practical assessment of coating application quality, coating adhesion is often considered. Standards include mechanical pull-off tests. However, the assessment of the adhesion of multi-coat polymeric systems can be complicated by the degradation of the separate coating components. Pull-off testing consists of the tensile force applied perpendicular to the coating surface. In non-ideal situations where coating compatibility is compromised, (i. e., with the development of coating blisters, peeling and disbondment) the developed strain in the coating layers may not be planar. Placement of the mechanical dolly for the pull-off testing would not necessarily reflect all the complications due to the non-uniform strain development and viscoelastic properties of the materials. Moreover, coating maintenance is costly, with an estimated annual steel bridge coating maintenance cost of $\$ 500$ million (NCHRP, 2007). So, interest lies in the improvement of coating durability. Literature suggested that thermodynamic wetting properties (i. e., contact angle, surface energy and work of adhesion) of polymeric surfaces can be useful to assess coating adhesion characteristics 
(Rudawska and Kuczmaszewski, 2006; Dmitruk, et. al., 2018; Johari et. al., 2016). So, it was tried to evaluate the adhesion of a nano-particle enriched zinc-rich composite coating (NPE-ZRP) to the steel substrates by the application of surface measurements to assess the degradation of multilayer (two-coat) coating compatibility with non-ideal steel substrate preconditioning. Different levels of pre-exposure conditions included high humidity, surface moisture and salt contamination with improper surface cleaning. The results of the traditional ZRP coating system (three-coat) are compared to address the influence of the incorporation of carbon nano-particles. A one-coat NPE-ZRP coating system was also assessed to identify the extent of degradation with exposure duration in different exposure environment. Finally, a statistical approach was used to correlate the coating durability parameters and identify the influence of nano-particles on coating durability.

\subsection{Material Characterization}

The sample's surface characteristics after pre-exposure to moisture and chloride contamination are described in detail in chapter 5. In general, the samples showed different levels of surface changes during the pre-exposure before the application of the repair coatings. The sandblasted samples showed more sensitivity to the pre-exposure conditions than hand cleaned samples (ie surface oxidation, rust). Figure 6.1 shows the contact angle measurement of the pre-exposed steel substrate. Humidity pre-exposed substrate shows a hydrophobic surface with a contact angle $>90^{\circ}$. Both sandblasted and hand cleaned samples showed the hydrophobicity due to the created surface roughness from the surface cleaning. Salt-contaminated samples showed a comparative lower contact angle due to the formation of iron oxide as water is adsorbed on the surface of 
metal oxide by hydrogen bonding to facilitate the formation of the adsorption complex.

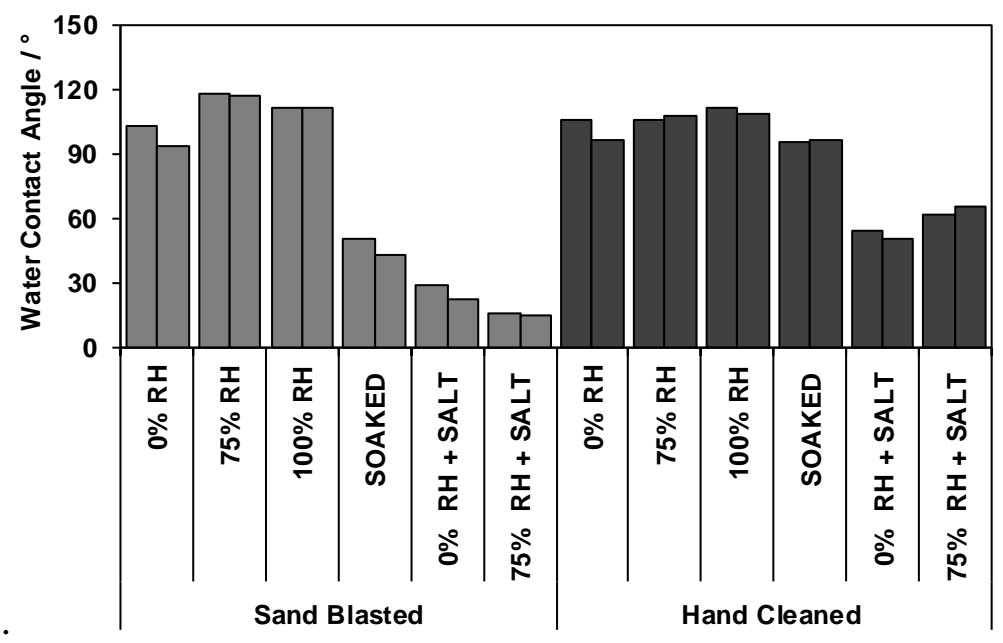

Figure 6.1. Contact Angle of the Pre-exposed Steel Substrate.

A characteristic of the tested samples after the repair coating application is summarized in Table 6.1. Representative metallographic cross-sections of NPE-ZRP and ZRP samples of the control sample prior to exposure are shown in Figure 6.2. There was generally a good anchor profile of the sandblasted steel substrate. The zinc primer sometimes showed interlayer defects. Also, hand cleaned samples often retained a significant remnant of old zinc primer (with zinc pigments generally showing bright reflection under plane light indicating non-oxidized state). After surface environmental pre-treatment in high humidity and salt contamination, a layer of rust formed immediately on the steel substrate below the zinc-rich primer (Figure 6.3). Moreover, osmotic blisters developed on the salt-contaminated samples due to the hygroscopic nature of the salt. 
Table 6.1. Characteristics of the Coatings under Study.

\begin{tabular}{cccccc}
\hline $\begin{array}{c}\text { Test } \\
\text { Group }\end{array}$ & $\begin{array}{c}\text { Steel } \\
\text { Substrate }\end{array}$ & Primer & Midcoat & Topcoat & $\begin{array}{c}\text { Total Thickness } \\
\text { (mils) }\end{array}$ \\
\hline \multirow{4}{*}{ A } & Sand blasted & $\begin{array}{c}\text { Zinc-rich silicate } \\
\sim 3 \text { mils }\end{array}$ & $\begin{array}{c}\text { Epoxy } \\
\text { midcoat } \\
\sim 5 \text { mils }\end{array}$ & $\begin{array}{c}\text { Polyurethane } \\
\text { topcoat } \\
\sim 2 \text { mils }\end{array}$ & $\sim 10$ \\
\cline { 3 - 6 } & Hand cleaned & $\begin{array}{c}\text { Zinc-rich epoxy } \\
\text { (with carbon nano-particles) } \\
\sim 5 \text { mils }\end{array}$ & No midcoat & $\begin{array}{c}\text { Epoxy } \\
\sim 4 \text { mils }\end{array}$ & $\sim 9$ \\
\cline { 2 - 6 } & $\begin{array}{c}\text { Zinc-rich epoxy } \\
\text { (with nano-particles) } \\
\sim 9 \text { mils }\end{array}$ & No midcoat & $\begin{array}{c}\text { Epoxy } \\
\sim 4 \text { mils }\end{array}$ & $\sim 13$ \\
\hline B & Sand blasted & $\begin{array}{c}\text { Zinc-rich epoxy } \\
\text { (with carbon nano-particles) } \\
\sim 7 \text { mils }\end{array}$ & No midcoat & $\begin{array}{c}\text { No } \\
\text { topcoat }\end{array}$ & $\sim 7$ \\
\hline
\end{tabular}
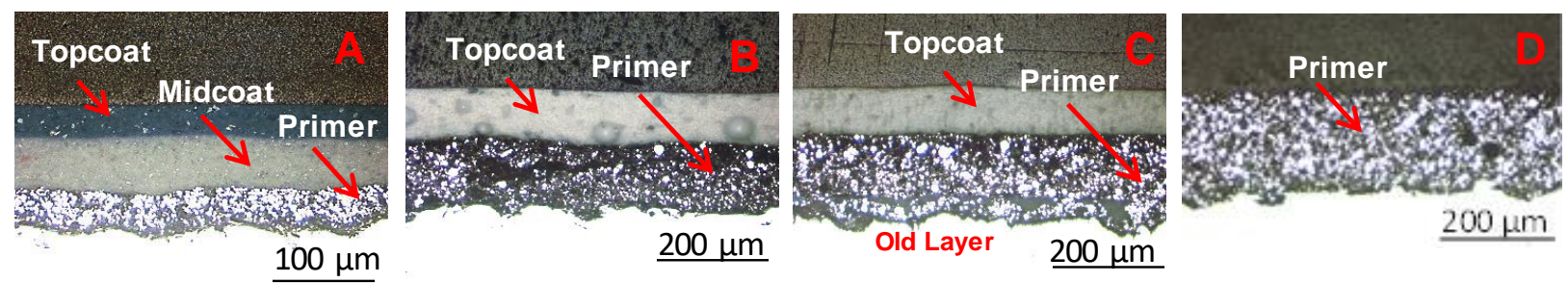

Figure 6.2. Optical Micrograph of NPE-ZRP Coating.

(A) Sand Blasted ZRP (3-Coat) (B) Sand Blasted NPE-ZRP (2-Coat) and (C) Hand Cleaned NPE-ZRP (2-Coat) (D) Sand Blasted NPE-ZRP (1-Coat)

Susceptibility to soluble salt varies with coating type, thickness, and exposure environment. The inherent level of interlayer primer imperfections, the introduction of non-ideal surface contaminants, exposure to moisture, and subsequent formation of coating defects would evidently impact the integrity of the coating interface adhesion. It was posed that both chemical interactions with water and contaminants as well as mechanical strain due to coating deformations would weaken the interface of the primer to the steel substrate and the primer to the topcoat. Conventional destructive pull-off testing would not discriminate these interactions and would rather only identify the point of weakest attachment. The identification of loss of material compatibility by this method 
remains a challenge. Nevertheless, quantification of pull-off strength with complementary identification of the coating layer that could be first separated will provide a base to assess the viability of surface wetting measurements to identify mechanisms of degradation of NPE-ZRP coating systems with adverse pre-conditioning with exposure.
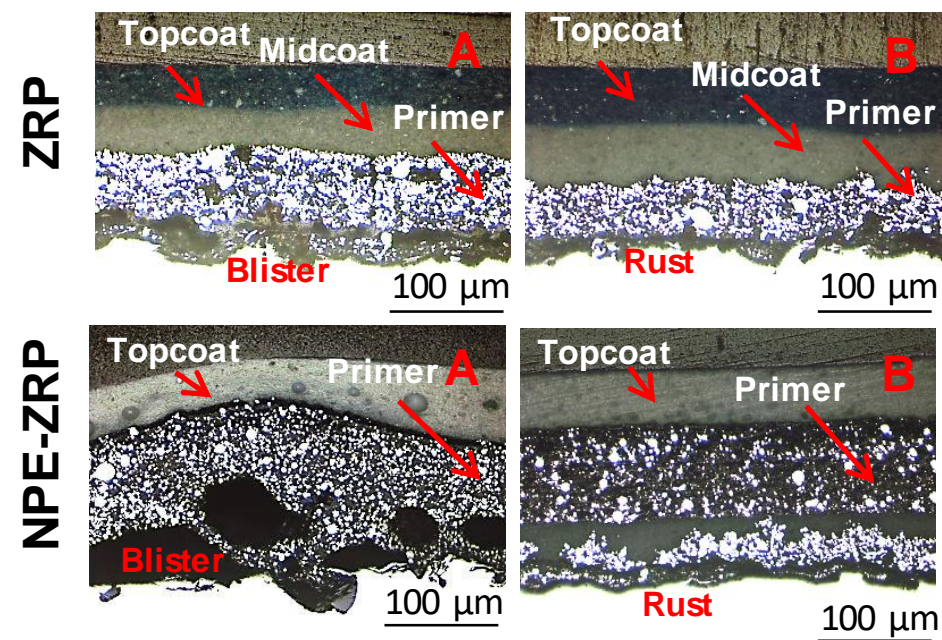

Figure 6.3. (A) Osmotic Blister formation on the salt contaminated samples.

(B) Rust Development at the interface of Humidity exposed samples.

\subsection{Coating Durability by Surface Measurements}

\subsubsection{Test Group A}

\subsubsection{Pull-off Strength}

Pull-off results were categorized as topcoat cohesive failure when both the dolly and the coupon surface had colored layers of the topcoat. The failure was considered a topcoat adhesive failure when the dolly had the topcoat material and the coupon retained the gray-colored primer. The failure was considered a primer cohesive failure when both the dolly and the coupon retained the primer. The failure was considered a primer adhesive failure when the dolly had the gray-colored primer and the coupon showed 
some level of grey that could be from the steel substrate, remnant mill scale, or initial surface rust. Tests that result in failure of the glue to attach the dolly are shown as minimum bound of pull-off strength. A representative picture of different failure modality is shown in Figure 6.4.
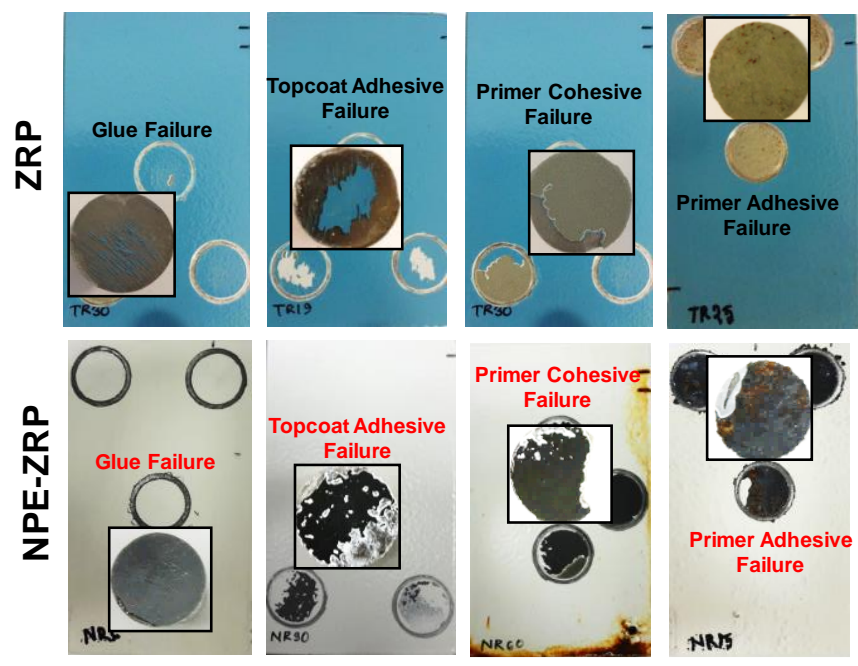

\section{Figure 6.4. Different Failure Modality of Pull-off Strength.}

In Figures 6.5-6.6, the minimum specified coating pull-off strength for structural steel coating systems specified by the Florida Department of Transportation (800 psi, $5516 \mathrm{kPa}$ ) is shown for comparison (FDOT, 2018). Prior to the exposure, the ZRP coating generally in the border of the minimum $5516 \mathrm{kPa}(800 \mathrm{psi})$ strength requirement for all test conditions indicating the overall marginal bond strength of the coating system. 


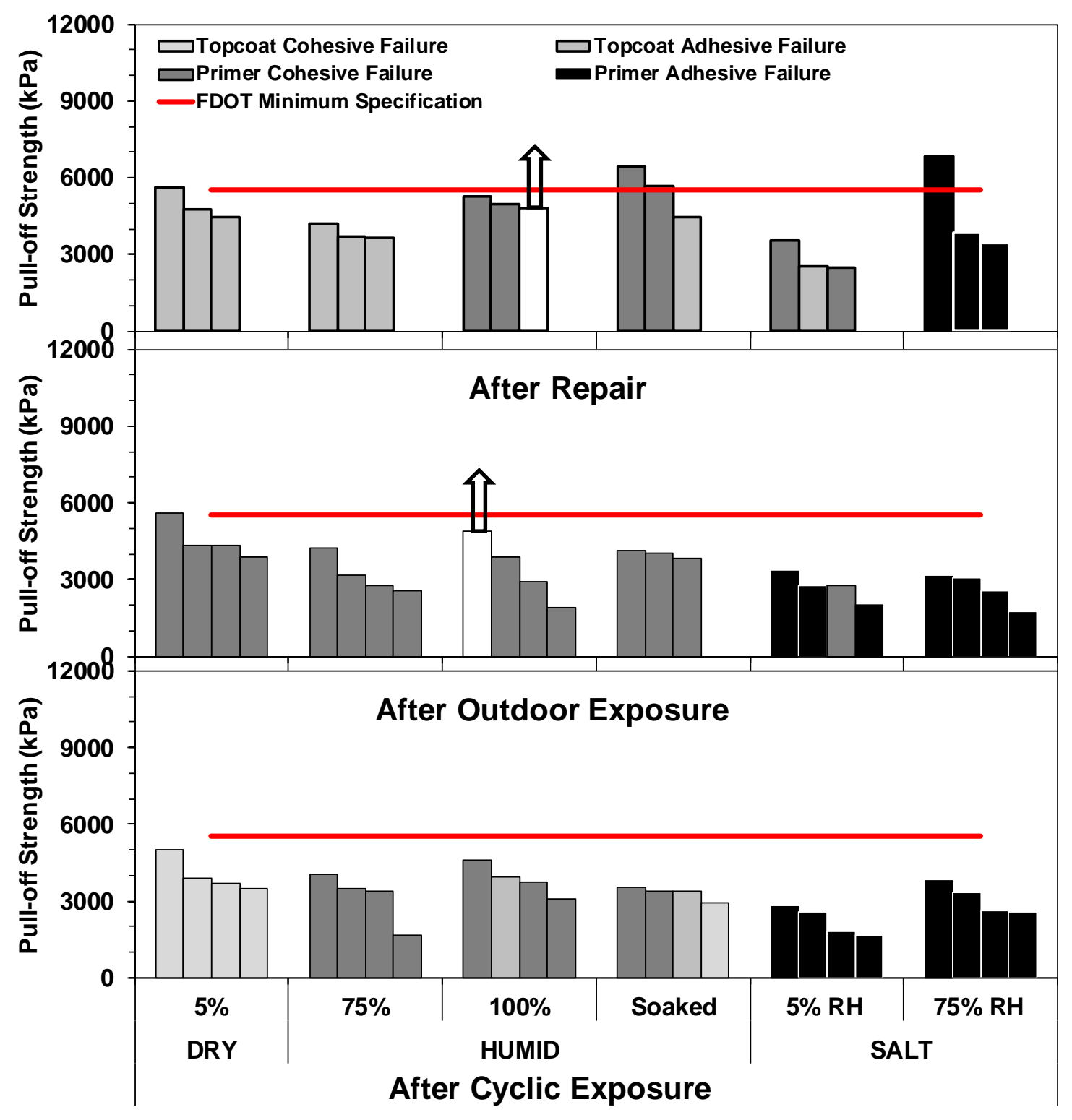

Figure 6.5. Coating Pull-off Strength of ZRP (Test Group A).

(Arrow indicates apparent higher value) 


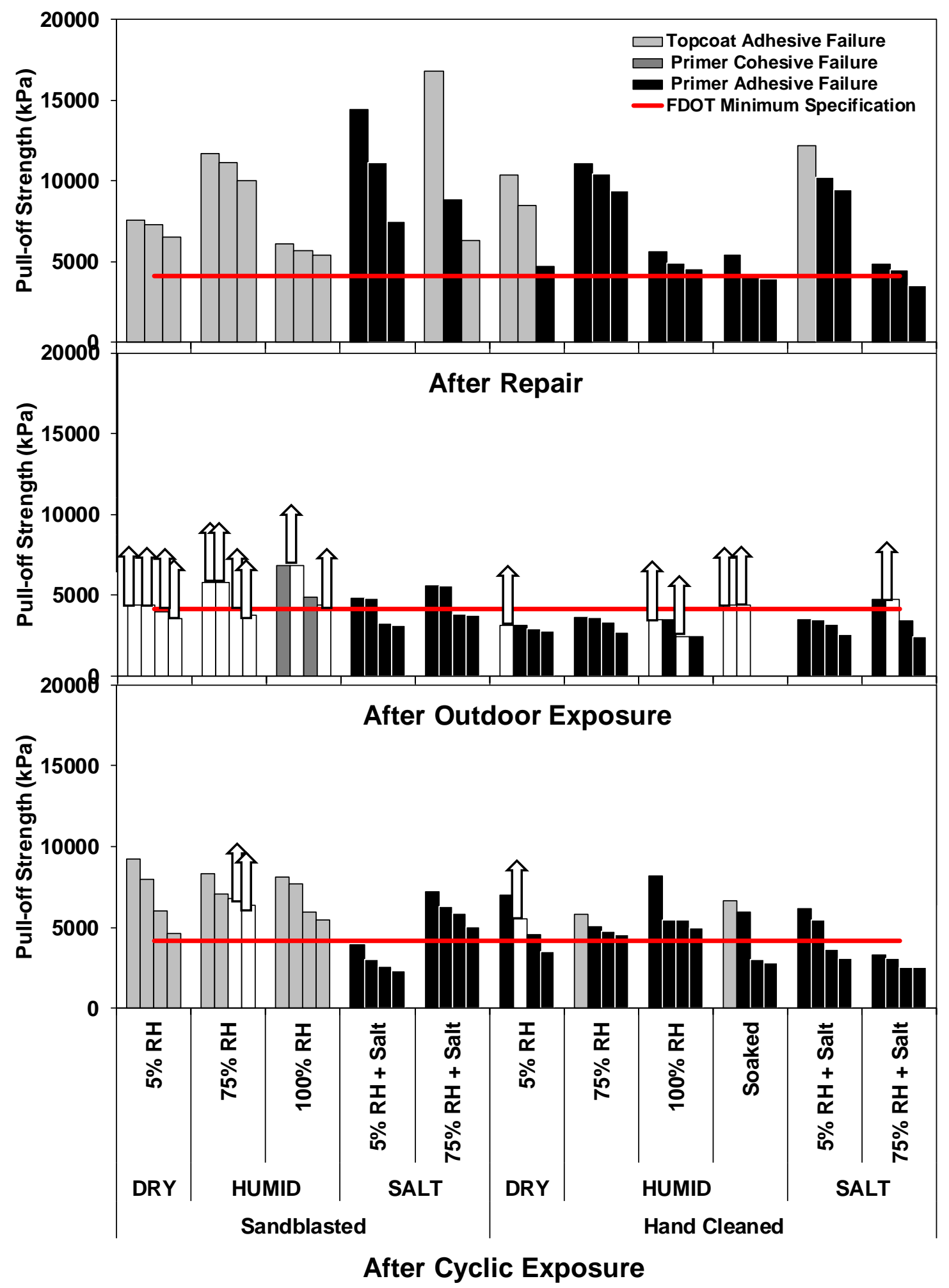

Figure 6.6. Coating Pull-off Strength of NPE-ZRP (Test Group A). (Arrow indicates apparent higher value) 
The pull-off strength testing resulted in comparative lower bond strengths for the samples exposed to high humidity (100\% RH and Soaked) and samples contaminated with salt by primer degradation, indicating the negative effect of the improper surface preparation. In the relatively benign $5 \%$ and $75 \%$ pre-exposure cases, the pull-off strength testing resulted in topcoat adhesive failure. After both outdoor and cyclic wet/dry exposure, all samples exhibited degradation in coating adhesion and loss in pulloff strength. In comparison to the coating pull-off strengths for samples immediately after coating application, testing resulted in failure at the primer for all test conditions, including the relatively benign 5\% and $75 \% \mathrm{RH}$ pre-exposure. Primer adhesive failure occurred for the salt-contaminated samples with lower strength.

Prior to the exposure, the NPE-ZRP coating generally exceeded the minimum $5516 \mathrm{kPa}(800 \mathrm{psi})$ strength requirement for all test conditions. Samples preconditioned with salt contamination and hand cleaning showed primer adhesive failure and topcoat adhesive failure was observed with the sand blasted 5-100\% RH preconditioned samples. It was apparent that the NPE-ZRP samples that were sandblasted and placed in preexposure condition 5-100\%RH had better adhesion than those samples that were hand cleaned or exposed to salt contamination. It is apparent that the level of surface oxidation (either iron or zinc oxidation from the previous layer) that formed in the preconditioning environments prior to the coating application would hamper the bond between the primer and the substrate. Salt contamination, as expected, resulted in poorer performance in coating adhesion.

Generally, all samples exhibited degradation in coating adhesion and loss in pulloff strength after both outdoor and wet/dry cyclic exposure. Comparative higher bond 
strength was observed for NPE-ZRP repaired samples than ZRP coated samples. After exposure, all ZRP samples and NPE-ZRP samples that were hand cleaned failed to meet minimum strength for all cases. NPE-ZRP samples that were sandblasted failed to meet minimum strength when chlorides were present, but the effect of preconditioning humidity alone (even up to $100 \%$ ) was not pronounced and abled to maintain its integrity with a strength higher than the specified.

\subsubsection{Wetting Property}
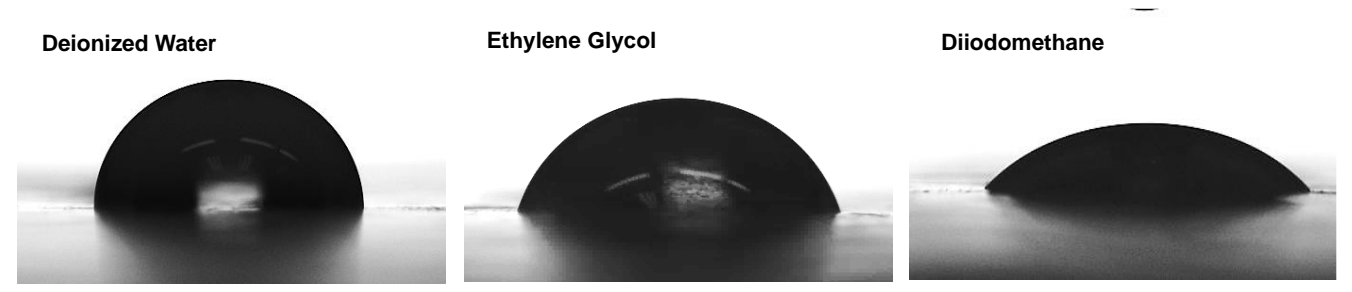

Figure 6.7 Liquid droplet on Test Group A Sample in As-received Condition.

Contact angles were measured on the repaired coated samples in as-received condition and after outdoor and cyclic exposure using three different wetting liquids (Deionized water, Ethylene Glycol, and Diiodomethane). The magnitude of the contact angle depends primarily on the ratio of the adhesive forces between liquid and solid and the cohesive forces of the liquid. When adhesive forces are less in relation to the cohesive forces, the contact angle is more than 90 degrees. Hydrophobic surfaces are considered anti-corrosives because they repel water; minimize the ingression of corrosive agents. In the as-received condition of NPR-ZRP coating, the contact angle using deionized water for all different pre-exposure conditions was measured $\sim 90^{\circ}$, indicating a hydrophobic surface that is not favorable for wetting. Whereas for ZRP coating in asreceived condition, the contact angle was measured $\sim 80^{\circ}$, indicating hydrophilic surface 
which is favorable for wetting. Regardless of the pre-exposure condition hydrophobic surface of NPE-ZRP coating further justified the improved barrier protection apparently due to the inclusion of nano-particles. Contact angle condition measured using Ethylene Glycol and Diiodomethane was $\sim 60^{\circ} \& \sim 50^{\circ}$ for NPE-ZRP and $\sim 60^{\circ} \& \sim 45^{\circ}$ for ZRP, respectively. Figure 6.7 shows the images obtained for the contact angle measurements for the three wetting liquids.

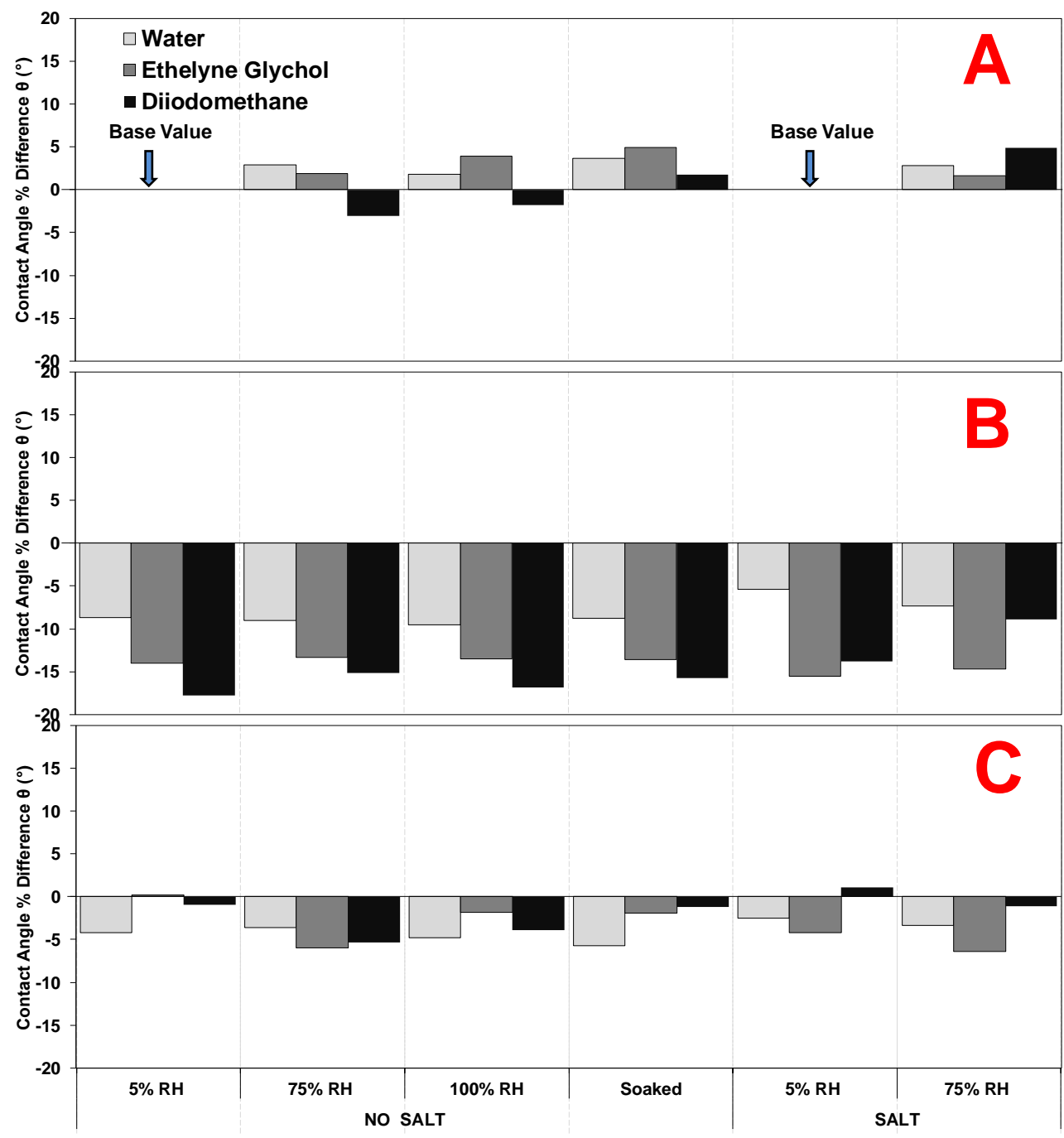

Figure 6.8. Percent Difference in Contact Angle for ZRP Samples (Test Group A). A) As received Condition. B) After Outdoor Exposure C) After Cyclic Exposure (Values are average of multiple readings on duplicate samples) 

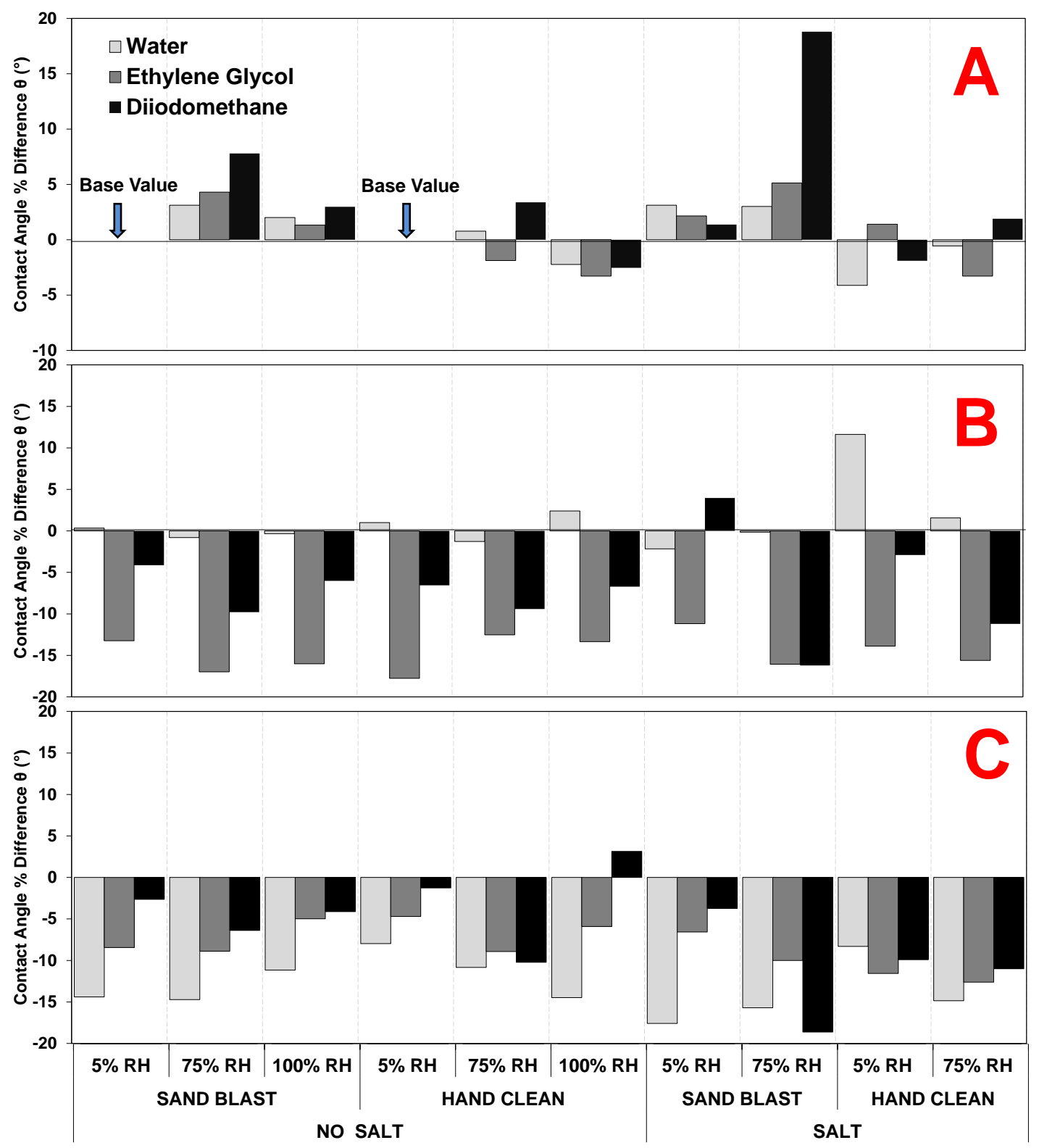

Figure 6.9. Percent Difference in Contact Angle of NPE-ZRP Samples (Group A). A) As received Condition. B) After Outdoor Exposure C) After Cyclic Exposure (Values are average of multiple readings on duplicate samples)

Figure 6.8 and 6.9 shows the percent change in contact angle for the different preexposure condition for ZRP and NPE-ZRP coating respecting. For as-received samples, the percent change was calculated with consideration of the control case (pre-exposure to $5 \% \mathrm{RH})$ as a reference. Percent change for each pre-exposure test condition was 
calculated as the normalized contact angle difference for samples before and after cyclic or outdoor exposure. In the as-received (environmentally pre-conditioned but nonexposed) condition for ZRP samples, measurement showed an increase in contact angle regardless of humidity and salt pre-exposure. After both outdoor and cyclic exposure, the testing resulted in a reduction in contact angle which is due to the degradation of the topcoat which more severe in the outdoor samples.

NPE-ZRP samples in as-received condition showed an increase in contact angle for the sand blasted and hand cleaned samples pre-exposed to humid condition. In contrast, salt contaminated samples showed an apparent decrease in contact angle, which is apparently due to the degradation of the coating layers. Indeed, coating blisters were visible on those samples. Furthermore, after both outdoor and cyclic exposure, the testing resulted in a greater reduction in contact angle, indicating degradation of the coating layers due to exposure.
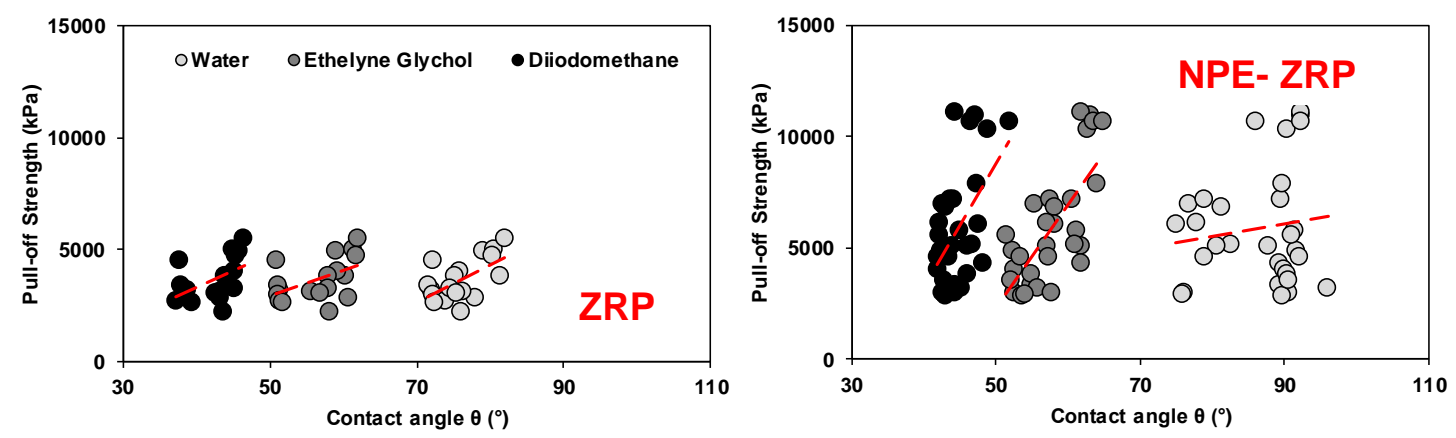

\section{Figure 6.10. Correlation between Contact Angle and Pull-off Strength (Group A).}

The pull-off strength versus contact angle plot for ZRP coating showed a general linear trend as shown in Figure 6.10. Generally, lower pull-off strength measurements corresponded to test samples with lower measured contact angles regardless of the wetting solution. The variation of contact angle data for each wetting solution is small 
$\sim 5^{\circ}$. Some scattered data point was also observed where lower pull-off strength observed with higher contact angle specifically for the salt contaminated samples where greater degradation observed at the steel/coating interface. The data points on the pull-off strength versus contact angle plot were somewhat scattered, and the correlation between the contact angle and pull-off strength was evidently related to the extent of hydrophobicity of the wetting solutions (Figure 6.10). Also, the variation of contact angle data for each wetting solution is small $\sim 10^{\circ}$. Nevertheless, there was a general trend of the measured contact angle and the corresponding measured pull-off strength. Generally, lower pull-off strength measurements corresponded to test samples with lower measured contact angles regardless of the wetting solution.
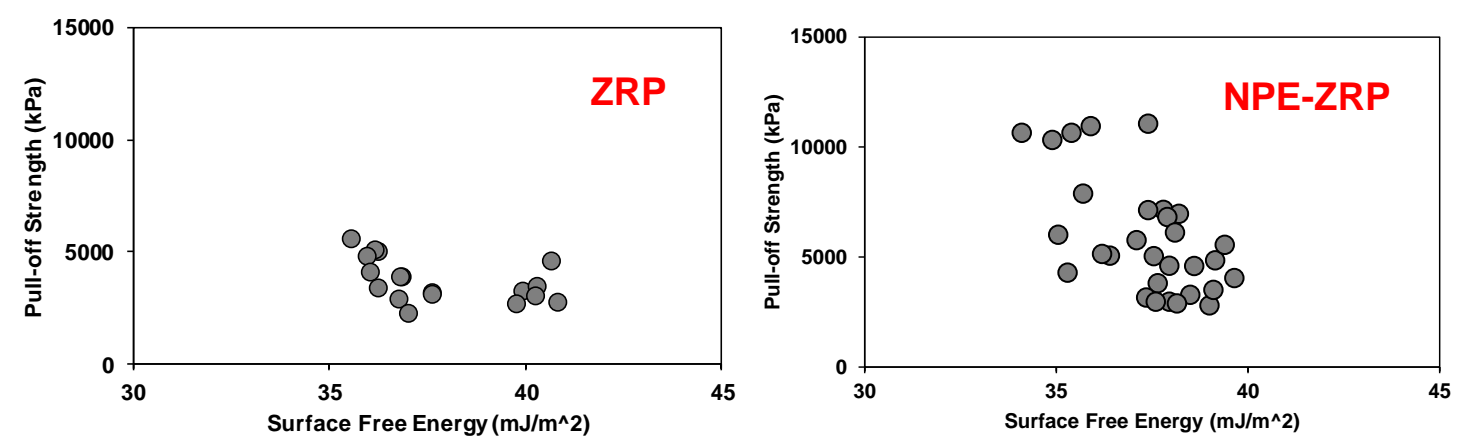

Figure 6.11. Correlation between Surface Energy and Pull-off Strength (Group A).

Due to the scatter related to the level of hydrophobicity of the wetting solution, surface energy calculations from the contact angle data were made. It was posed here that good coating adhesion would relate to high surface tension and correspondingly lower surface energy. For ZRP coating mostly lower pull-off strength was observed regardless of the surface energy posed the weak bond strength of the coating system (Figure 6.11). There was a general trend of higher surface energy with the corresponding reduction in pull-off strength for NPE-ZRP coating as shown in Figure 6.11. 
Clear differentiation in the hydrophobicity between the two-coating system clearly indicates the influence of incorporation of carbon nanotubes which increase the barrier characteristics of the NPE-ZRP coating system. Also, the reduction in contact angle indicates the degradation of the coating system due to salt contamination as well as insufficient surface preparation (hand cleaning). For ZRP low contact angle regardless of pre-exposure indicates less bond strength and reduction after environmental exposure indicates further degradation. So, the results are promising for a more detail evaluation of coating layer degradation. The role of undercoating iron oxidation, zinc pigment consumption, moisture levels within the coating, as well as mechanical strain on the coating is expected to be relevant and is expected to account for some of the experimental data scatter. An important geometric characteristic of practical application in terms of the surface wetting parameters is the relatively thick primer layer, application of midcoat and topcoat. The steel/coating interface would likely be in part obscured in the surface wetting in such conditions. Also, the primer to midcoat and midcoat to topcoat interface may play a contributing factor. Therefore, additional tests (Test Group B) with only the primer layer of NPE-ZRP coating were tested.

\subsubsection{Test Group B}

\subsubsection{Pull-off Strength}

The NPE-ZRP samples in Test Group B consist of only the primer layer and did not have any adverse environmental preconditioning. Most of the pull-off tests resulted in failures of the glue. The lower bound pull-off strength of samples in the as-received condition was more than 10,000 kPa. Furthermore, after 12 months of outdoor exposures, pull-off testing did not result in coating adhesive or cohesive failures, but the mechanical 
dolly after testing typically showed evidence of residual particles that appeared to be removed from the primer top surface. The residual particles were thought to be the surface zinc oxidation product with exposure. For the salt-fog exposure, the measured pull-off strength values were lower than the initial as-received value and cohesive failure of the primer was observed on samples exposed for 12 months.

\subsubsection{Wetting Property}

Contact angle measurement of NPE-ZRP Test Group B coating without topcoat and substrate preconditioning is presented in Table 6.2. As there was no protection from a topcoat and the zinc pigments from the exposed coating surface were consumed during the environmental exposure by creating micro galvanic cells as well as for better connectivity with the steel substrate. This change in surface characteristics evidently was reflected in the change in contact angle with the time of exposure. In the outdoor exposure, the contact angle increased to a value as high as $\sim 120^{\circ}$ consistent with the effects of zinc oxidation products likely filled capillaries and pores with oxidation products. The salt-fog exposed samples after 4 months of exposure showed similar behavior as an increase in contact angle but after 12 months of exposure, a significant reduction in contact angle was observed as shown in Figure 6.12.

Table 6.2. Contact Angle of NPE-ZRP Sample without Topcoat.

\begin{tabular}{ccccc}
\hline \multirow{2}{*}{ Exposure } & \multirow{2}{*}{ Duration } & \multicolumn{3}{c}{ Contact Angle $\boldsymbol{\theta}\left({ }^{\circ}\right)$} \\
\cline { 3 - 5 } & & Water & Ethylene Glycol & Diiodomethane \\
\hline Asreceived & 0 & 71.25 & 60.2 & 53.3 \\
\hline Outdoor & 4 months & 115 & 64.8 & 38 \\
\hline Outdoor & 12 months & 118.5 & 57.8 & 38.8 \\
\hline Salt-Fog & 4 months & 101.1 & 61 & 50.8 \\
\hline Salt-Fog & 12 months & 54.7 & $\sim 5$ & $\sim 13.2$ \\
\hline
\end{tabular}

*Values are average of multiple readings on duplicate samples 


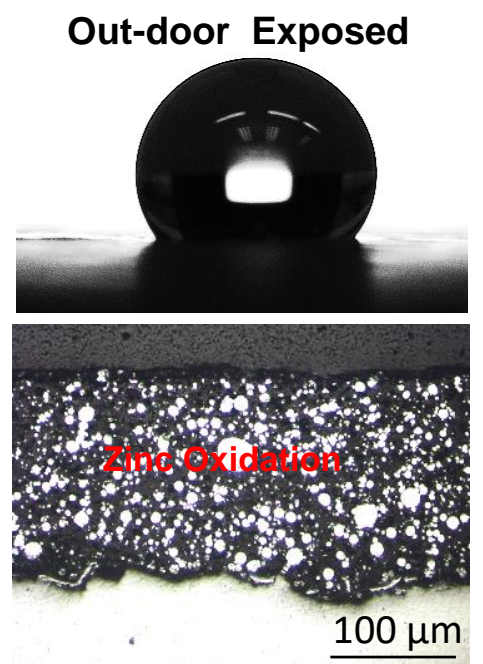

Salt-fog Exposed
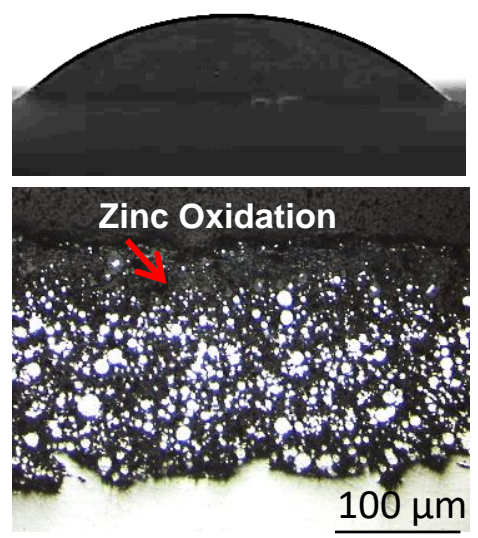

Figure 6.12. Liquid droplet on Test Group B Sample after Exposure.

The results largely relate to the changes on the surface as zinc particles are oxidized in the aggressive environments and the subsequent surface roughening and pore filling. Due to these additional surface parameters, comparison to Test Group A samples to explore the application of surface wetting parameters cannot be easily compared. Nevertheless, the large changes in contact angle as the NPE-ZRP primer is exposed to the environment (and results in apparent degradation of the coating materials) are positive indicators that surface wetting measurements can be used for assessment of coating durability.

Pull-off testing, in general, showed that hand cleaned NPE-ZRP samples failed to meet minimum strength for all cases after exposure. No preconditioning humidity exposure (even up to 100\%) effect was observed on sandblasted NPE-ZRP samples but failed to meet minimum strength when there was chloride contamination. Quantification of pull-off strength with identification of NPE-ZRP coating layer of degradation by surface wetting property measurements was thought to reveal the role of undercoating 
iron oxidation, zinc pigment consumption, moisture levels within the coating, as well as mechanical strain on the coating as a base to assess the degradation mechanisms of NPEZRP coating. After both outdoor and cyclic exposure reduction in contact angle measurements was evident and there was a general trend of higher surface energy with the corresponding reduction in pull-off strength. But the measured contact angle data showed a very small variation in range with the samples consisted topcoat. The measured small change in the contact angle of the two-coat system can be due to the degradation of the top exposed layer. Samples without topcoat to verify the influence of thick practical coating layer as well as primer-to-topcoat interface on wetting parameter showed a distinct change in contact angle as zinc particles are oxidized in the aggressive environments and the subsequent surface roughening and pore filling. Nevertheless, the changes in contact angle as the NPE-ZRP primer is exposed to the environment are positive indicators that surface wetting measurements can be used for assessment of coating durability.

\subsection{Statistical Approach to Find Coating Durability}

The long-term durability of zinc-rich coating systems depends on the coating characteristics (zinc pigment amount, size, distribution), proper application (surface preparation) and bond strength with the steel substrate. A statistical approach was used to develop a relationship between primer thickness, pull-off strength and zinc consumption that can provide a better understanding of interactions among test variables. Based on the model, a durability range was proposed by the interdependent parameters.

\subsubsection{Multiple Regression Analysis}

To develop the statistical model, both NPE-ZRP and ZRP coated steel samples 
were used. NPE-ZRP and ZRP coatings were applied over sand blasted steel substrate, exposed to different levels of humidity level $(5 \%, 75 \%$ and $100 \% \mathrm{RH})$ before the coating application. After the coating application samples were exposed to cyclic wet/dry exposure for $\sim 5$ months. Different coating parameters such as zinc content, zinc size distribution ratio, primer thickness, zinc consumption, humidity pre-exposure, and pulloff strength measurements were investigated by bivariate analysis and multi-collinearity check to identify the significant parameter for durability model development by multiple regression analysis. Multicollinearity can affect any regression model and it occurs when two or more variables overlap so much that their effects are indistinguishable in the model outcome. Bivariate correlation between every two variables was analyzed to check for multicollinearity and the results are as follows.:

\section{Investigated Parameters:}

- Significant: Time, Thickness, Pull-off, Zinc consumption.

- Non-significant: Humidity, zinc content, Zinc size ratio.

During the wet/dry cyclic exposure current data was recorded over time as represented in Figure 6.13. A comparative smaller corrosion current was observed for the NPE-ZRP samples. 

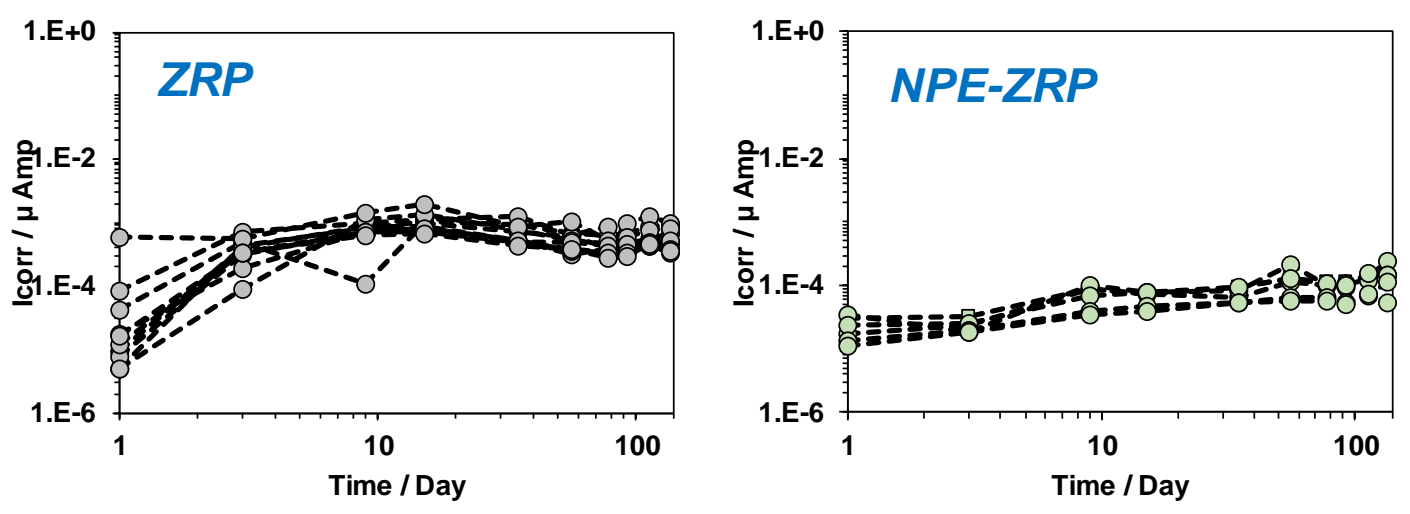

Figure 6.13. Corrosion Current Evolution the Cyclic Wet/dry Exposure.

Figure 6.14 shows the distribution of $\%$ cross-sectional zinc area for both coatings in as-received condition for multiple samples. Zinc content was greater in the crosssection area for ZRP coating. For further analysis of zinc consumption, it was assumed that zinc density was $25 \%$ for NPE-ZRP and $35 \%$ for ZRP coating in as-received condition.

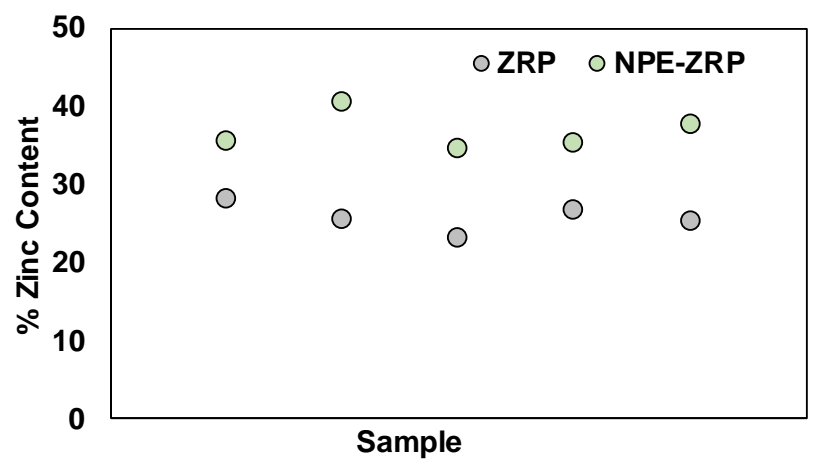

Figure 6.14. Distribution of \% Cross-sectional Zinc Area.

Equivalent mass loss was calculated from the cumulative charge using Faradaic conversion. Total zinc content was calculated for each different sample by considering its zinc distribution, primer thickness and the exposed area to wet/dry exposure. Zinc consumption and associated \% zinc decrease were estimated considering the calculated 
mass loss for along the exposure duration by Faradaic conversion as shown in Figure 6.15. As can be seen from the $\%$ zinc decrease that zinc consumption was comparatively smaller for NPE-ZRP coating.
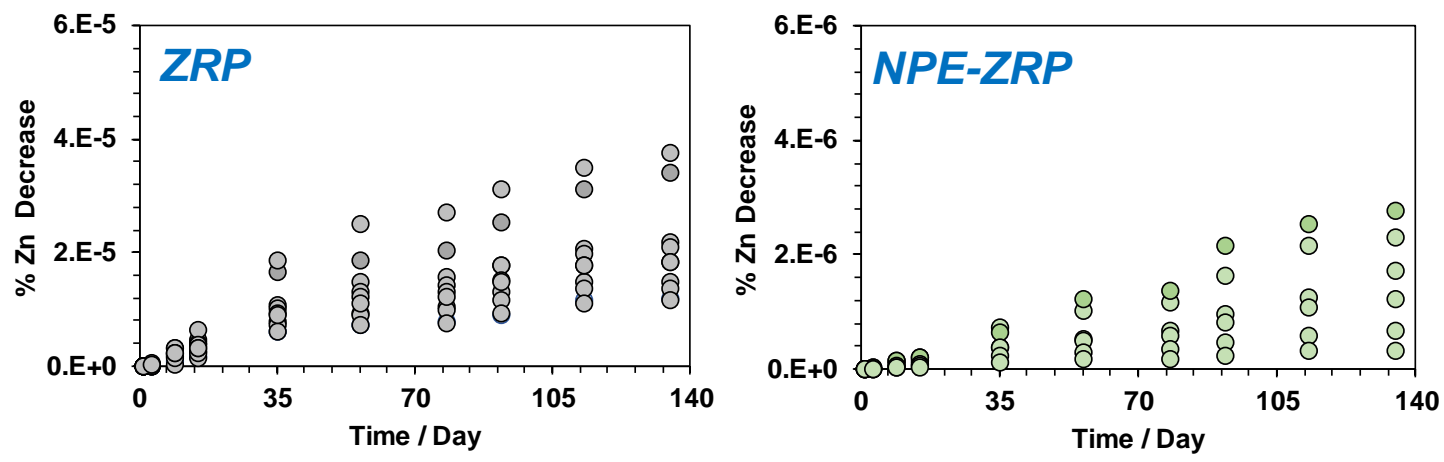

Figure 6.15. \% Zinc Decrease Calculated from Cumulative Charge.

Based on the preliminary analysis of defining significant parameters, a statistical model was developed to correlate primer thickness, pull-off strength to the zinc consumption over time by multiple regression analysis. Based on the previous results (described in chapter 5) it was assumed that pre-exposure to RH didn't have an effect on the coating performance and zinc consumption variations due to different humidity were not considered in the regression analysis. Regression analysis was performed on the performance data of both coating systems to identify and understand the above relationships. This correlation would be specific to the cyclic exposure condition. The relationship between the variables can be understood with the numerical correlation on how the variation of parameters affects each other with time. Multiple regression analysis of the above combinations of variables was conducted using R Language, and correlations with R-squared values higher than 0.6 were identified. The analysis information is as follows: 


\section{Dependent variable:}

- Pull-off Strength

- $\quad$ Thickness

- Time

\section{Proposed zinc consumption,}

$$
\begin{gathered}
Y=M_{1}+M_{2} \times T+M_{3} \times D+M_{4} \times P \\
M_{1}, M_{2}, M_{3}, M_{4}=\text { Regression Coefficients } \\
T=\text { Time } \\
D=\text { Primer Thickness } \\
P=\text { Pull - off Strength } \\
Y=\% \text { Zinc Consumption }
\end{gathered}
$$

Table12. Coefficients from Multiple Regression Analysis for Both Coatings.

\begin{tabular}{c|c|c|c|c|c|c}
\hline \multirow{2}{*}{ Coefficients } & \multicolumn{2}{|c|}{ Estimate } & \multicolumn{2}{c|}{ t value } & \multicolumn{2}{c}{ Pr(>|t|) } \\
\cline { 2 - 7 } & ZRP & NPE-ZRP & ZRP & NPE-ZRP & ZRP & NPE-ZRP \\
\hline Intercept & $1.301 \mathrm{e}-05$ & $7.022 \mathrm{e}-07$ & 2.988 & 2.756 & $0.00356^{* *}$ & $0.00788 * *$ \\
\hline Day & $1.582 \mathrm{e}-07$ & $1.137 \mathrm{e}-08$ & 14.021 & 11.661 & $<2 \mathrm{e}-16 * * *$ & $<2 \mathrm{e}-16 * * *$ \\
\hline Thickness & $-5.826 \mathrm{e}-08$ & $-6.923 \mathrm{e}-09$ & -2.324 & -7.015 & $0.02222 *$ & $3.27 \mathrm{e}-09 * * *$ \\
\hline Pull-off & $-1.984 \mathrm{e}-09$ & $8.667 \mathrm{e}-11$ & -1.996 & 2.692 & $0.04881 *$ & $0.00934 * *$ \\
\hline
\end{tabular}

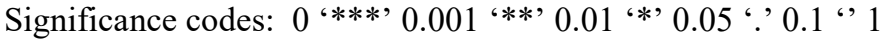

Proposed Equation for Zinc Consumption:

\section{ZRP Coating,}

$$
\begin{gathered}
Y=1.301 E^{-05}+1.582 E^{-07} \times T-5.826 E^{-08} \times D-1.984 E^{-09} \times P \\
R^{2}=0.6805
\end{gathered}
$$




\section{NPE-ZRP Coating,}

$$
\begin{gathered}
Y=7.022 E^{-07}+1.137 E^{-08} \times T-6.923 E^{-09} \times D+8.667 E^{-11} \times P \\
R^{2}=0.772
\end{gathered}
$$

The appropriateness of the multiple regression model can be tested by the T-test.

From table 1, it can be clearly seen that for both coatings model all regression coefficients (dependent variables) are significantly based on the P-value. Based on the regression model equation a triangular relationship was developed between the dependent variables as shown in Figures 6.16-6.17 for both coatings. To develop the durability range with better performance a zone was created as shown in the red region, by the limiting primer thickness specified by the manufacturer $(150 \mu \mathrm{m}$ for ZRP and $200 \mu \mathrm{m}$ for NPE-ZRP) and minimum pull-off strength ( 5000 kPa, FDOT maintenance manual). For ZRP coating system, none of the samples fell into the red region due to failure to meet the minimum pull-off strength requirements. All the samples fall into the blue region.

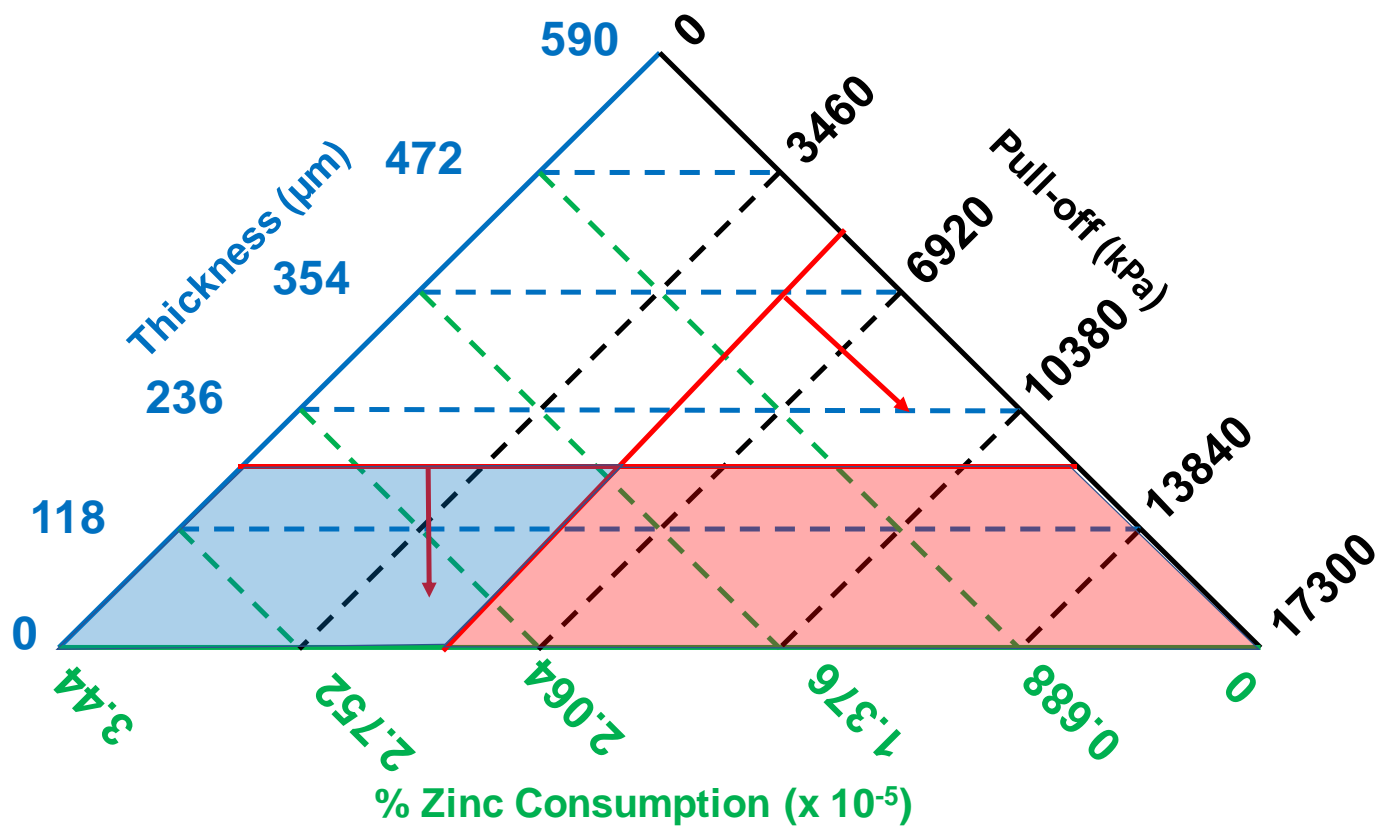

Figure 6.16. Proposed Range for Durability of ZRP Coating. 
For NPE-ZRP coating system, a wide red region was identified with very high pull-off strength value as glue failure was observed for some of the samples. But the majority of the samples fall into the blue region. The difference in durability range between NPE-ZRP and ZRP coating systems also clearly indicates the influence of incorporation of carbon nano-particles by higher pull-off strength and lower zinc consumption with improved performance.

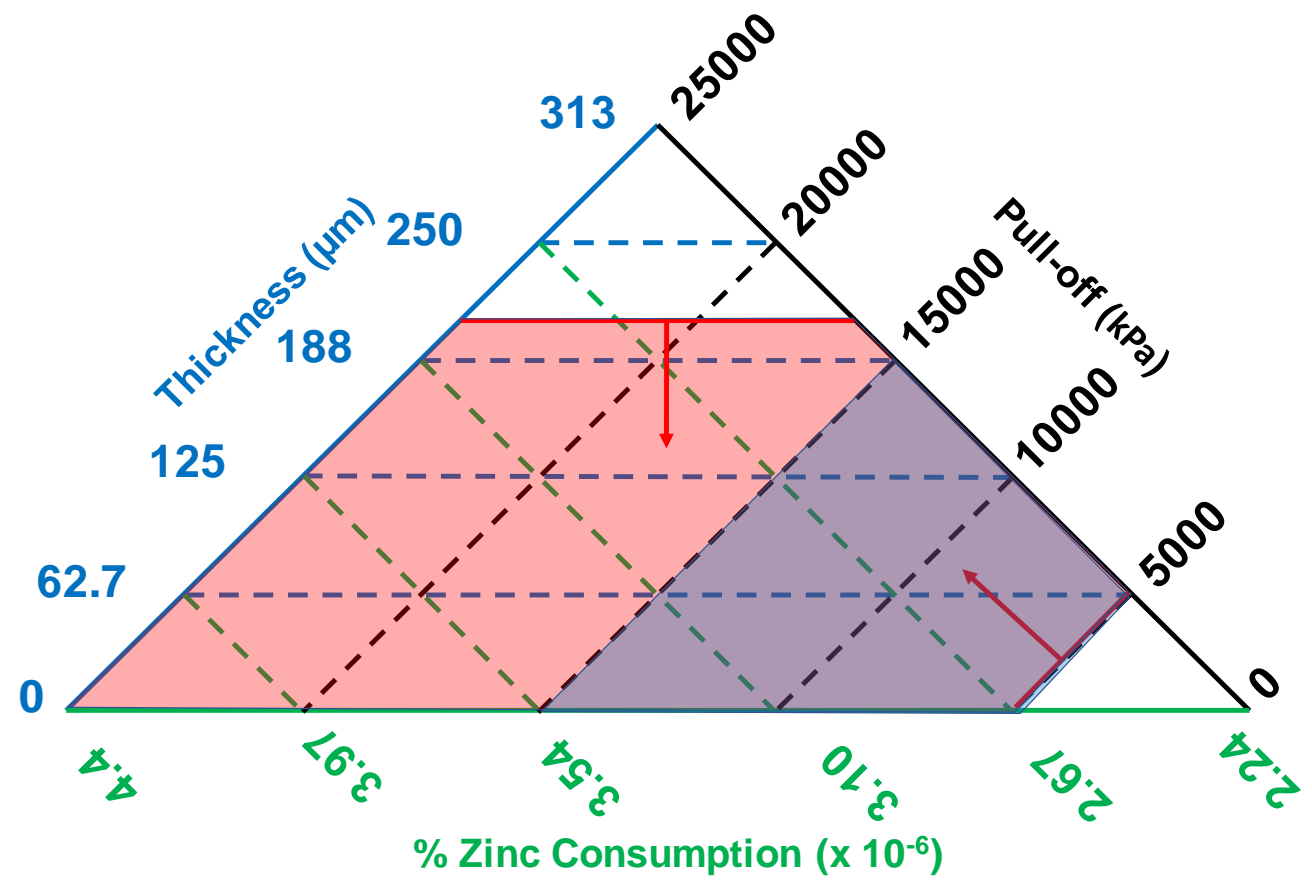

Figure 6.17. Proposed Range for Durability of NPE-ZRP Coating. 


\section{CHAPTER 7}

\section{ZINC CONSUMPTION MECHANISM}

\subsection{Introduction}

Zinc-rich primer (ZRP) based coating systems are widely used to protect steel infrastructure from aggressive exposure environments since the 1980's. These coating systems provide corrosion protection of the steel substrate by both barrier and sacrificial mechanism (Abreu et. al., 1999). The physical barrier to environmental exposure develops from the polymeric coating layer and embedded zinc pigments provide the sacrificial cathodic protection when exposed. To overcome the limitation of using a high volume of zinc content which is vulnerable for the primer bond strength to the steel substrate, carbon nano-particles are incorporated due to its beneficial mechanical and electrical characteristics. In this effort, a nano-particle enriched zinc-rich primer coating system (NPE-ZRP) was evaluated to provide corrosion protection. A tradition zinc-rich coating system (ZRP) was also evaluated to compare the overall performance of the NPE-ZRP coating system for our highway steel bridge application.

\subsection{Role of Nano-Particles on Barrier and Cathodic Protection}

Samples were evaluated in aggressive salt-fog, water immersion and outdoor exposure (beach and inland). Both of the coating systems exhibited good barrier protection when it was free of inherent coating defect (pinhole) as seen from all the unscribed samples from all different exposure (presented in chapters 4 and 5). No visual indication of major degradation was observed for the samples except some level of surface roughening or staining observe which is apparently due to the degradation of polymer layer (mostly in outdoor exposure by photochemical reaction and hydrolysis in 
prolonged moisture exposure) and oxidation of the exposed zinc pigments. Due to the porous nature of the polymeric material the electrolyte diffuses through the coating layer and activates the zinc pigments. By the development of micro galvanic cells between zinc pigments or cathodic polarization between the zinc pigments and steel, substrate forms stable zinc oxide which blocks the inherent pore spaces and enhanced the barrier protection further. Figure 7.1 shows the OCP evolution of both NPE-ZRP and ZRP unscribed samples during 60 days of exposure in $3.5 \% \mathrm{NaCl}$ aqueous solutions. OCP of repaired samples was also shown to compare the effect of probable deficiency in surface cleaning. The initial OCP of all samples was $\sim-0.0 \mathrm{~V}_{\mathrm{SCE}}$ except NPE-ZRP samples for application in new infrastructures. Comparative more active potential for those samples was due to the direct exposure of zinc pigments to the electrolyte as there was no addition barrier from the topcoat like another set of samples. The relatively noble potentials and the low corrosion current indicate barrier protection provided by all the coated samples. The differentiation between the two coating systems performance is that even with the thinner coating thickness NPE-ZRP (only primer layer) coating showed a similar potential evolution as ZRP coating which consists of two additional layers of barrier above the primer layer. Again, the repaired NPE-ZRP samples (two-layer) showed consistent evolution of noble potential whereas ZRP coating (three layers) showed much more active potential with the diffusion of electrolyte. Comparative enhanced barrier performance of NPE-ZRP coating even with fewer layers and less thickness is apparently due to the contribution of the nano-particles which are filling the inherent pore spaces of the epoxy matrix. 

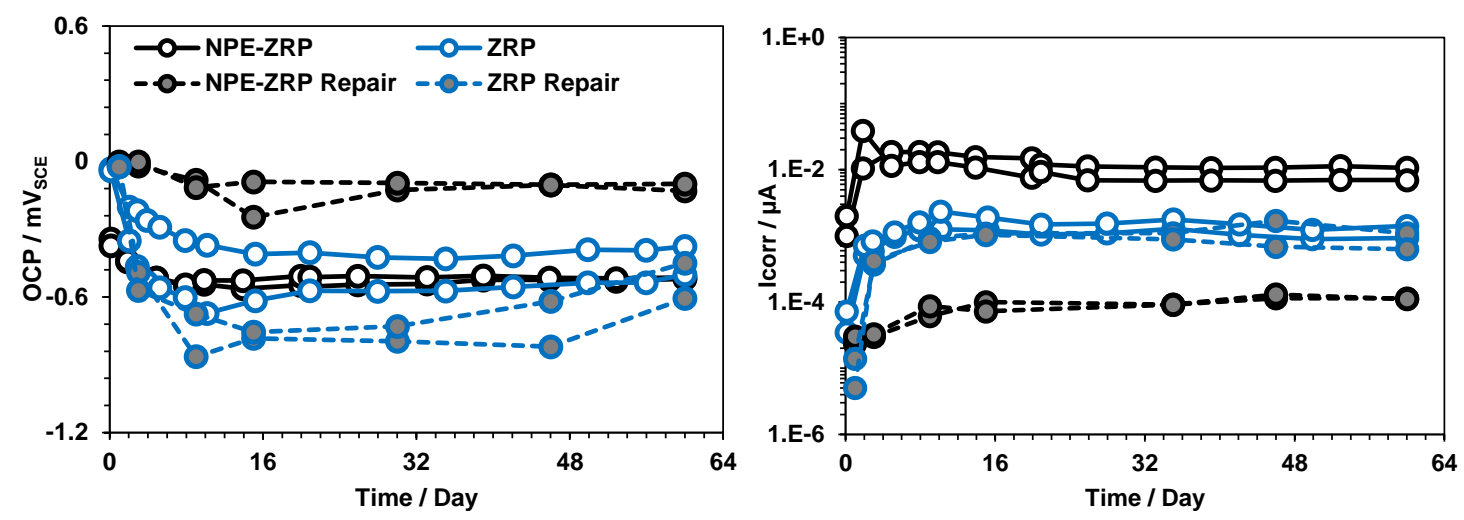

Figure 7.1. OCP and Corrosion Current over Time.

The evolution of more corrosion currents for the NPE-ZRP samples without topcoat specifies the enhanced continuity of the zinc pigments. This performance can also be verified by the early behavior of the scribed samples presented in chapter 4 where the early faster activity was observed for the exposed zinc pigments of the NPE-ZRP samples. Within two days of exposure, the OCP shifted to the sustained noble potential of $\sim-0.7 \mathrm{~V}_{\mathrm{SCE}}$ indicates less effective but some extent of beneficial galvanic coupling to the steel substrate. The associated high current after the apparent shift in the potential to passive-like conditions apparently in part accounts for the steel corrosion activity. Slightly higher corrosion current was measured for NPE-ZRP in comparison to ZRP coating apparently due to the enhanced conductivity for the incorporation of carbon nanotubes with less amount of zinc percentage as NPE-ZRP contains $\sim 75 \%$ zinc content whereas in ZRP the amount is $85 \%$. With the consumption of zinc pigments a layer of oxide forms around the zinc pigments (Figure 7.2) which makes them unconducive for further galvanic coupling. Point analysis was also done to verify the formation of zinc oxide around the zinc pigments. EDS point mapping confirms the formation of zinc oxide by detecting oxygen for points 1 and 3 . Whereas point 2 indicates pure zinc which no 
detection of on that point. Formation of zinc oxide and associated pore-blocking enhanced the barrier protection of the coating system which was confirmed by the EIS analysis (Chapter $4 \& 5$ ). So, with the less amount of zinc content for NPE-ZRP coating showed the similar galvanic coupling of ZRP coating with higher zinc content confirms enhanced galvanic protection by the inclusion of carbon nano-particles. Enhanced barrier protection of the NPE-ZRP coating with less coating thickness also verified the positive impact of the inclusion of carbon nano-particles. But the depletion of cathodic protection within two days of exposure when the steel substrate is exposed suggested that the inclusion of carbon nano-particles with the reduced zinc from traditional zinc content for prolonged cathodic protection was not achieved. Indeed, effective cathodic protection was valid during the first day of exposure which was confirmed the OCP potential of $\sim 1.0 \mathrm{~V}_{\mathrm{SCE}}$, (which is potential of active zinc) and no visual indication of rust development. So apparent more improvement in the composition of the coating is required either with the increment of zinc content or efficient dispersion of the nanoparticles to provide effective continuity which will lead to prolonging cathodic protection. 

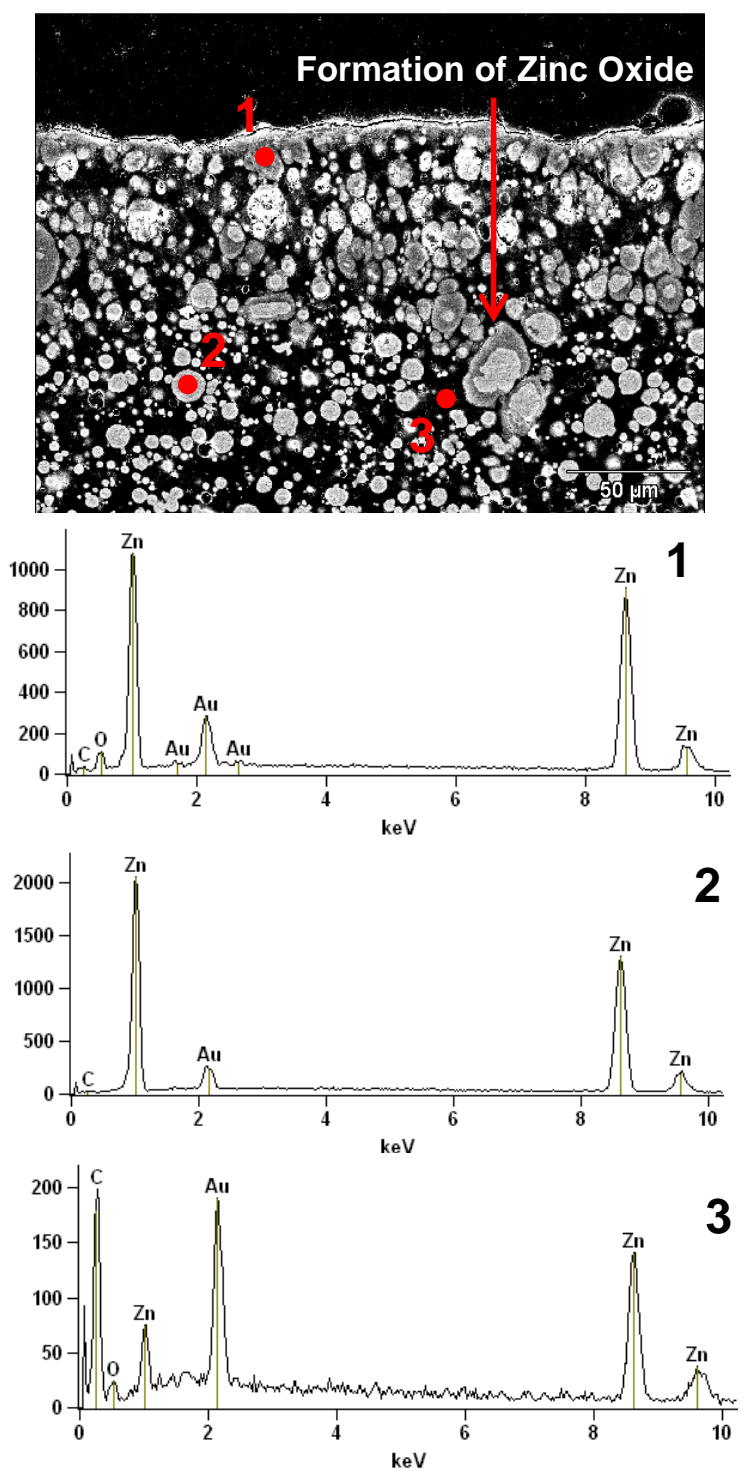

Figure 7.2. EDS Point Mapping on NPE-ZRP Sample.

\subsection{Role of Nano-Particles on Coating Bond Strength}

NPE-ZRP samples exposed to different environmental exposure exhibit higher mechanical performance compared to traditional ZRP coating. After both outdoor (inland and beach) exposure, NPE-ZRP coating pull-off testing generally resulted in glue failures indicating apparent overall integrity with no adhesive or cohesive failures of the primer layer. However, the coating pull-off test after exposure gave some indication of minor 
coating degradation by the residual particles on the dolly surface were thought to be the surface zinc oxidation product deposited on the primer surface from the exposed zinc pigments. But the salt-fog exposed samples indicated possible degradation of NPE-ZRP in extended exposures (after 12 months) to moist salt environments by the primer cohesive failure. EDS elemental mapping was done on the salt-fog exposed samples to identify the extent of degradation. Figure 7.3 shows the EDS mapping on the top part of the 24 months exposed NPE-ZRP sample and Figure 7.4 shows the EDS mapping on the bottom part of the same sample.
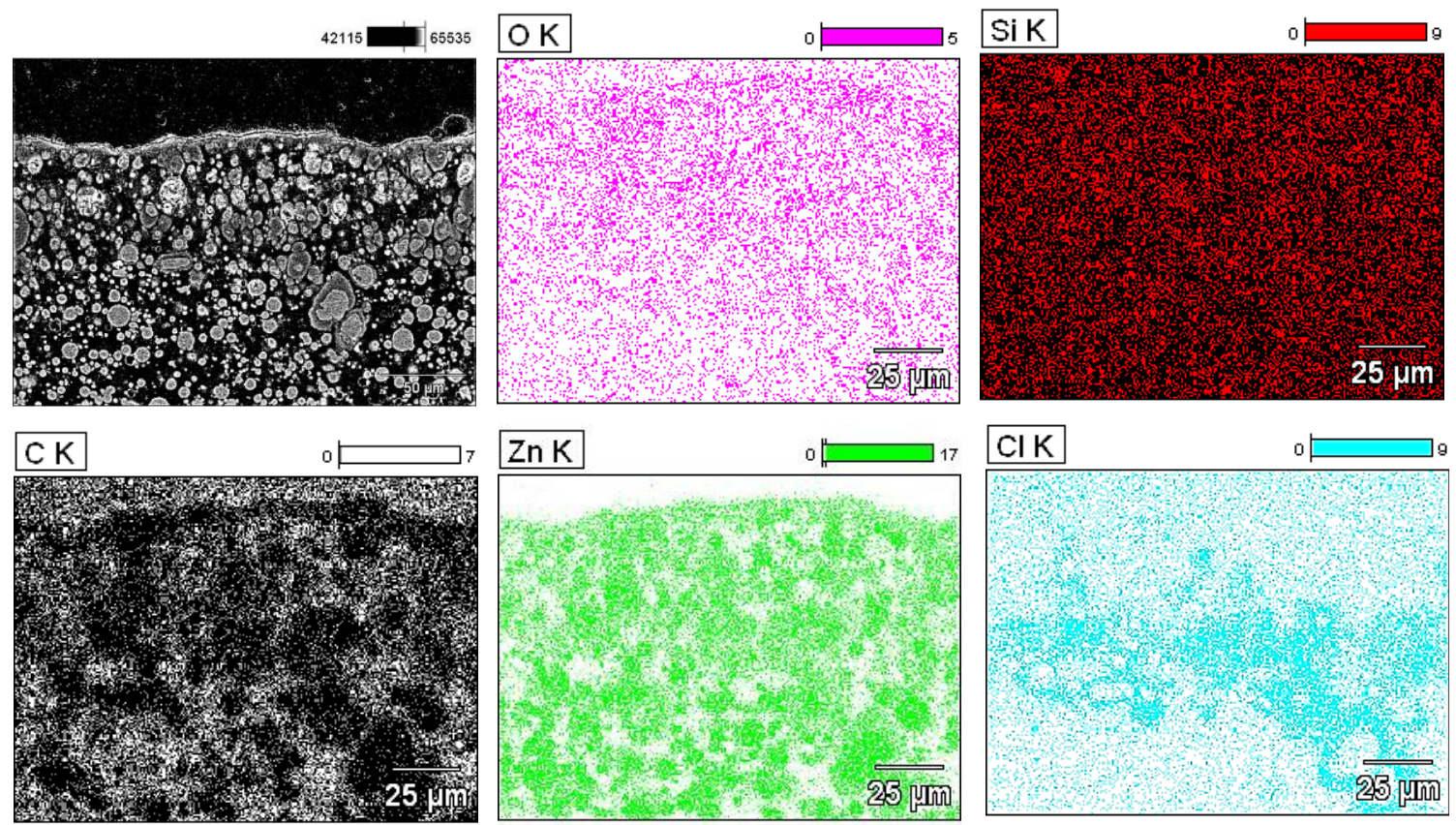

Figure 7.3. EDS Mapping of Salt-fog Exposed NPE-ZRP Unscribed sample (Top). 

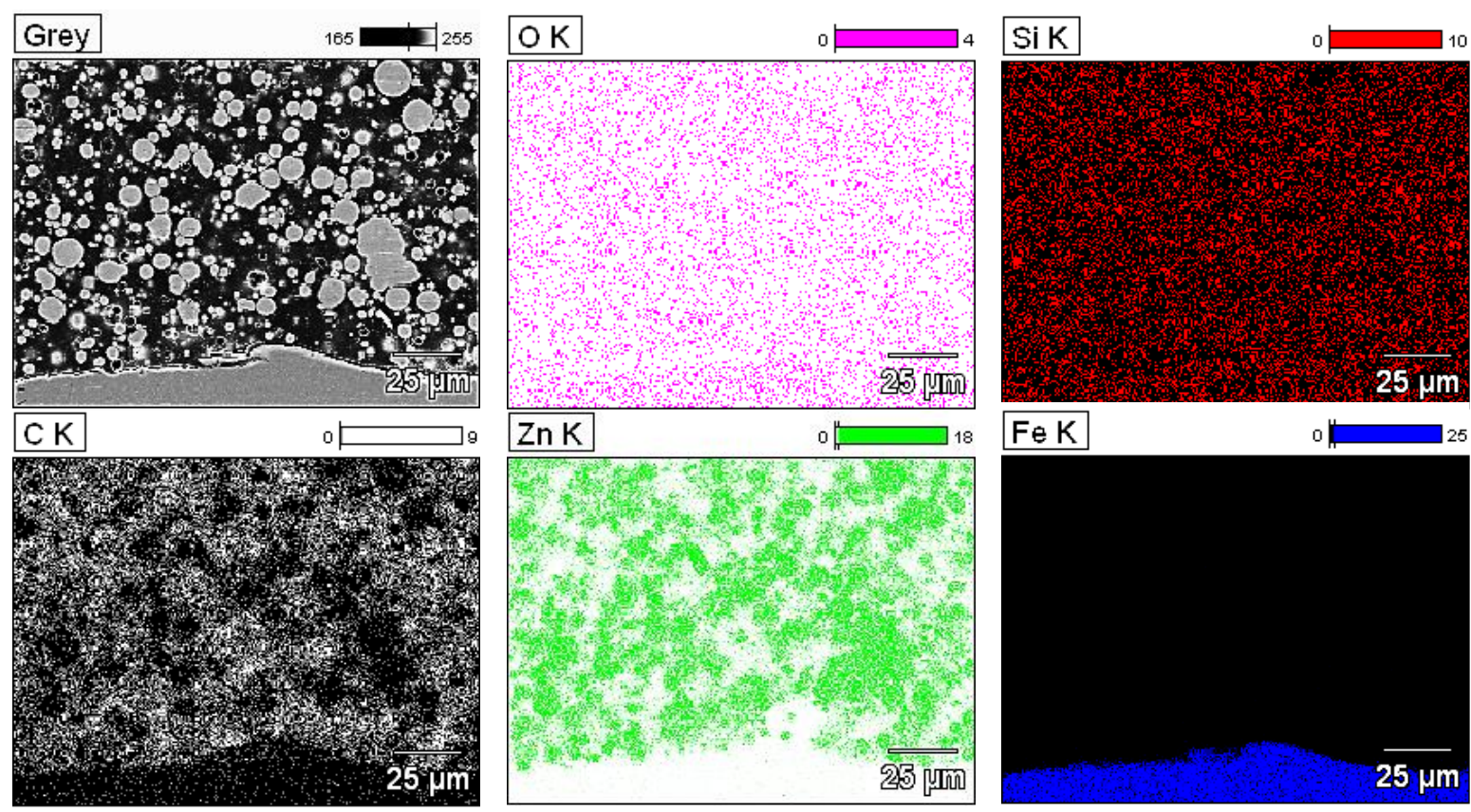

Figure 7.4. EDS Mapping of Salt-fog Exposed NPE-ZRP Unscribed sample (Botm).

The formation of zinc oxide is evident at the top part of the EDS mapping by more concentrated oxygen availability in that region and also by the chlorine ring formation around the zinc pigments. XRD analysis also confirmed the zinc oxide formation by the reaction with chlorine. The formation of zinc oxide was evidently continuing from the exposed top surface towards the bulk primer layer with the passage of electrolyte. Formation of heavy zinc oxide along the cross-sectional thickness makes the primer layer brittle which is the reason for primer cohesive failure. With further exposure when electrolyte and chloride reach the substrate formed of zinc further reactions apparently cause the primer adhesive failure.

ZRP coating initially either resulted in topcoat adhesive or primer cohesive failure. With the exposure aggressiveness when electrolyte diffuses through the coating layers zinc consumption continues along with the steel/primer interface. Further, the 
cathodic reaction at the steel interface increases the $\mathrm{pH}$, which leads to the disbondment of the primer layer from the steel substrate which is clearly defined in the SEM image and EDS mapping (Figure 7.5).
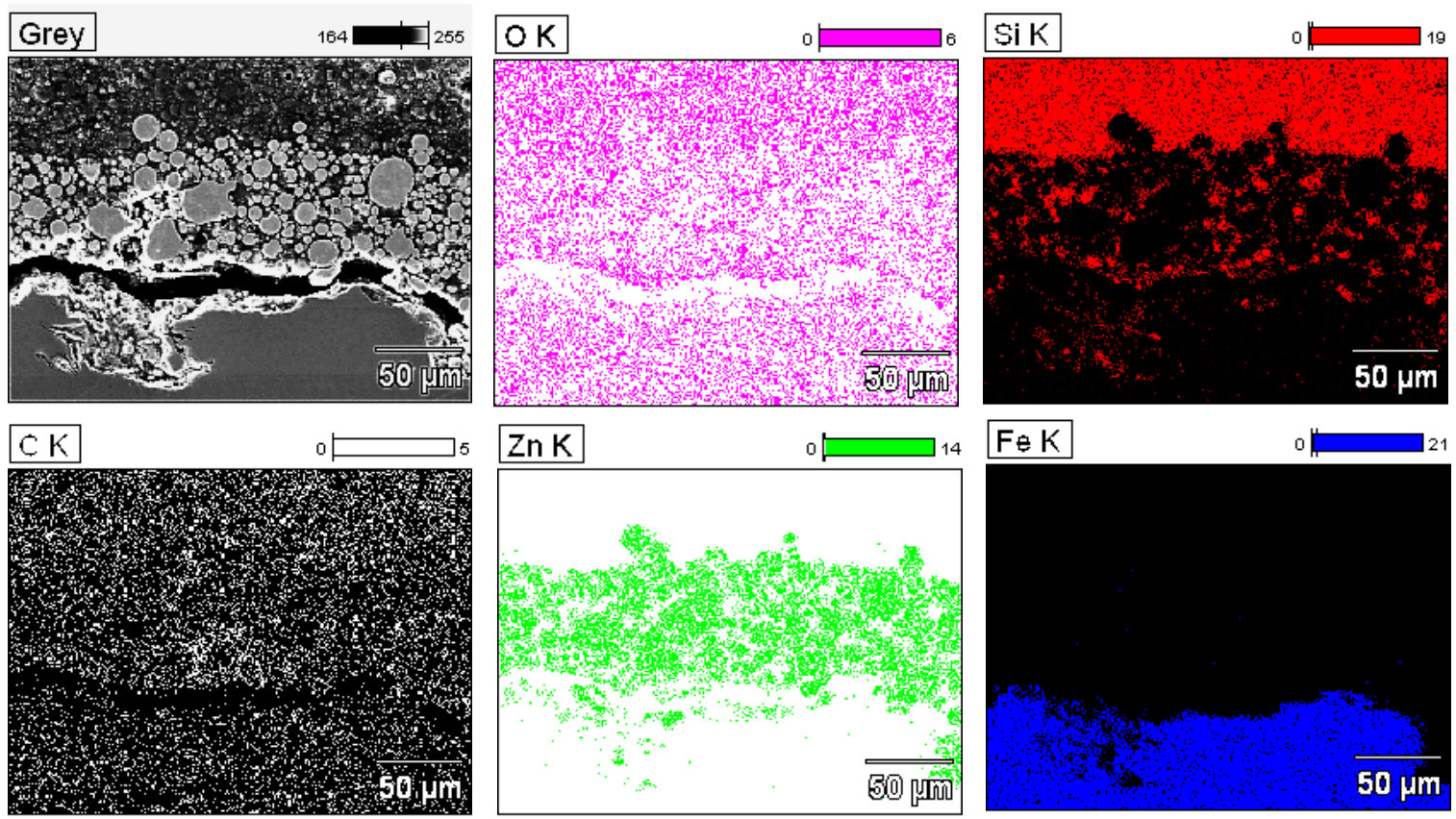

Figure 7.5. EDS Mapping of a Salt-fog Exposed ZRP Unscribed sample.

Figure 7.6 and 7.7 shows the EDS mapping of 60 days solution immersed scribe sample for NPE-ZRP and ZRP coating. Similar behavior in terms of zinc consumption was observed for both coatings. For NPE-ZRP coating after some initial consumption around the coating defect, zinc consumption continues from the bulk primer layer and no disbondment occurs at the steel primer interface which implicates the beneficial effect of carbon nano-particles in the primer matrix. Inclusion of carbon nano-particles enhanced the mechanical performance of the primer matrix which increased the cohesive as well as adhesive bond the primer with the steel substrate. But for ZRP coating after some initial consumption around the coating defect, zinc consumption continues along with the 
steel/primer interface and resulted in disbondment indicates the poor mechanical

performance of the primer bond with the steel substrate due to formation of zinc oxide.
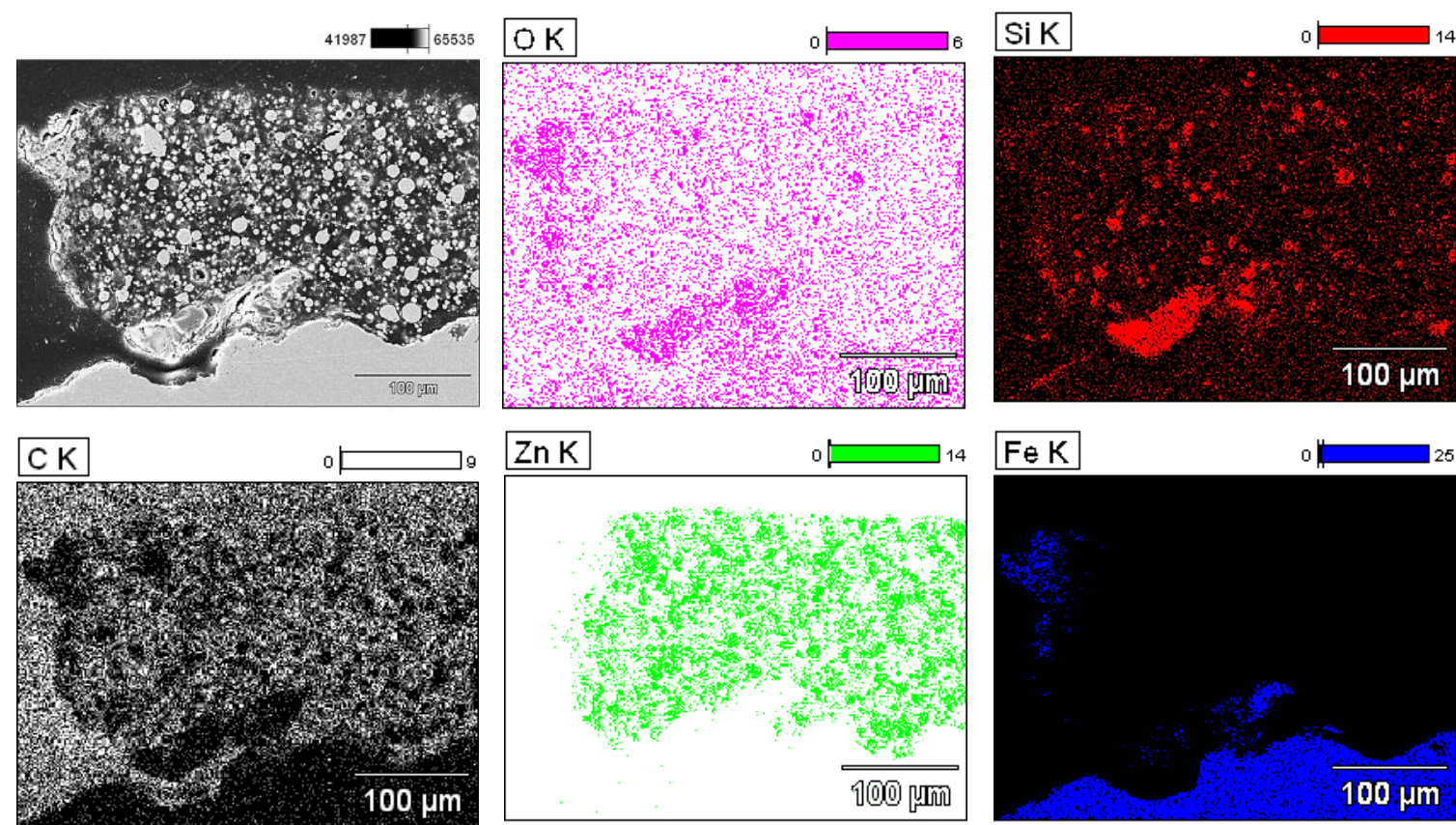

Figure 7.6. EDS Mapping of a Solution Immersed NPE-ZRP Scribed sample.
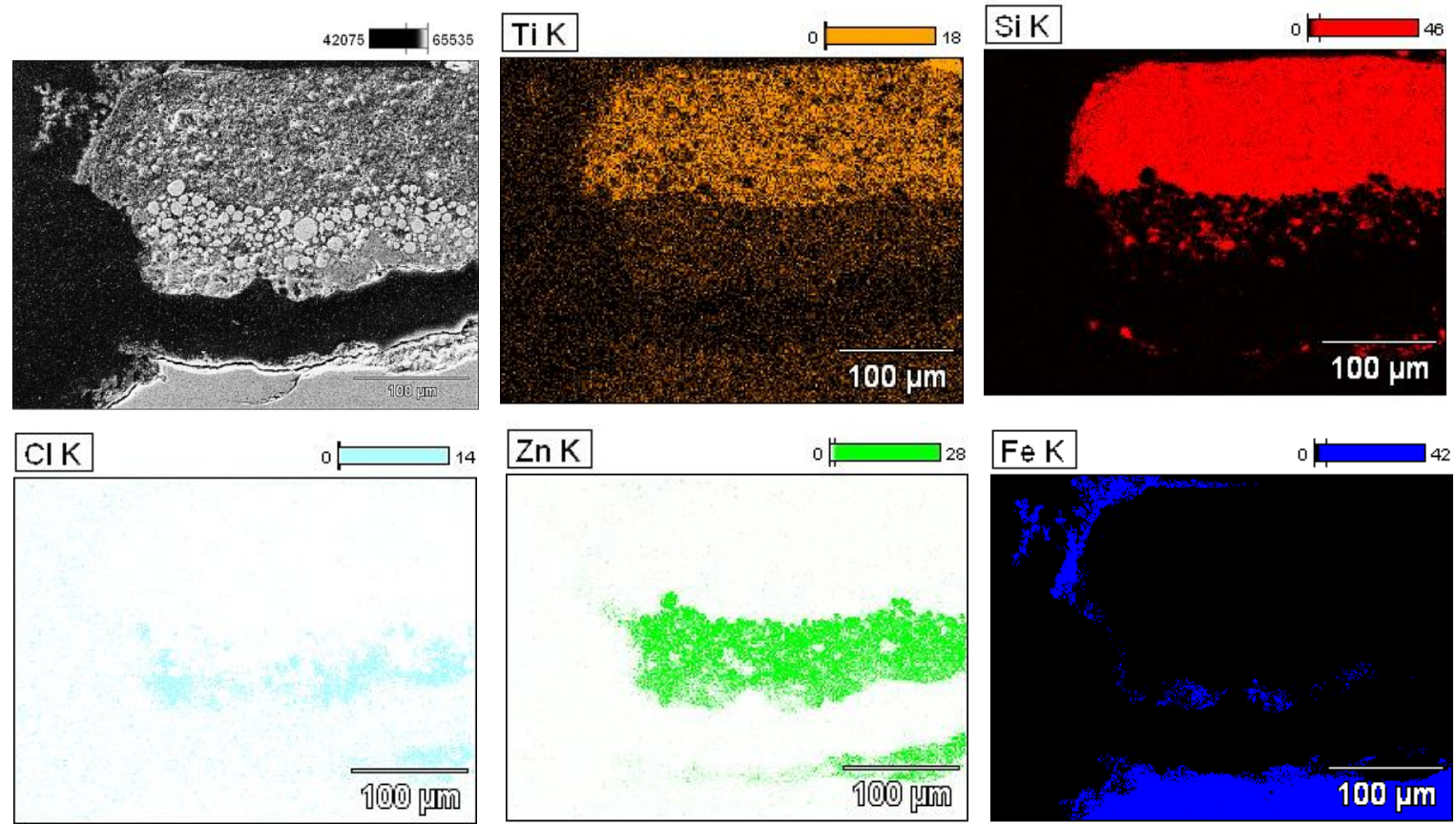

Figure 7.7. EDS Mapping of a Solution Immersed ZRP Scribed sample. 


\section{CHAPTER 8}

\section{CONCLUSIONS}

A nano-particle enriched zinc-rich epoxy primer (NPE-ZRP) coating system was exposed in the outdoor and salt-fog environment to access the integrity of the coating system in humid and aggressive marine bridge environment. The NPE-ZRP was assessed to identify probable enhanced coupling of the zinc pigments to the steel substrate and enhanced mechanical performance in comparison to the conventional zinc-rich primer (ZRP). Electrochemical tests were done in immersion conditions to get further information about the degradation mechanism of the coating. Deficient surface preparation was incorporated to address if the incorporation of carbon nano-particles can mitigate the associated adverse impact during coating repair application. Repaired samples were exposed to outdoor and aggressive alternate wet/ dry exposure to access the coating durability. The findings of those test results are summarized in the following paragraphs

- $\quad$ NPE-ZRP coating characterized as a two-coat coating system containing zinc-rich epoxy primer followed by an epoxy topcoat. Metallic zinc pigments are widely dispersed in the epoxy matrix reinforced by carbon nano-particles. Cross-sectional zinc density is greater in traditional inorganic zinc-rich primer. The distribution of the zinc size is not well graded for NPE-ZRP samples. Carbon nano-particles reinforced the continuity between zinc pigments instead of the high-volume content of traditional ZRP coating.

- The intact coating system provides superior barrier performance for both NPEZRP and ZRP coating as seen by OCP \& EIS analysis. The porous nature of the 
epoxy matrix allows electrolyte penetration from the exposure environment which facilitates the activation of zinc pigments from the primer layer and the associated formation of zinc oxide further enhanced barrier protection. Comparative improved barrier performance was observed for NPE-ZRP coating system even with fewer coating layer as well as less thickness, outwardly due to the influence of inert carbon nano-particles which block the inherent pore spaces in the epoxy matrix.

- Upon exposure through defect region, the duration of high zinc activity (<$\left.1000 \mathrm{mV} V_{\mathrm{SCE}}\right)$ in salt solution was very short ( 2 days) in OCP condition for both NPE-ZRP and ZRP coating. OCP shift to more noble potentials apparently indicates apparent less effective galvanic protection.

- Poor electrical conductivity for the zinc corrosion products that formed around the zinc pigments apparently limits the electrical contact between zinc pigments and the steel substrate which leads to poorer galvanic protection as well as to subsequent steel corrosion by the reduction of active zinc to steel area.

- For unscribed samples, initial zinc consumption was dominant along the top exposed surface for NPE-ZRP coating whereas for ZRP coating it was mostly at the steel primer interface. For scribed samples after initial zinc consumption around the periphery of the coating defect, for NPE-ZRP coating, dominant galvanic contributions attributed from the bulk zinc pigments apparently due to improved galvanic coupling by the nano-particles whereas for ZRP coating zinc consumption continues along with the coating/steel interface. The difference in zinc consumption modality for NPE-ZRP coating from the bulk primer layer 
confirms the enhanced connectivity of the zinc pigments through the carbon nanoparticles even with less amount of zinc content compared to ZRP coating.

- Higher pull-off strength was observed for NPE-ZRP coating apparently due to the uniform dispersion of carbon nano-particles in the epoxy matrix which enhanced the cohesive bond and interlocking within the pore spaces of the steel substrate which facilitate improved adhesive strength by the high strength nano-particles.

- Apparent enhanced conductivity and enhanced coating mechanical adhesion due to enhanced connectivity of zinc pigments afforded by the nano-particles allows greater galvanic contribution from the zinc pigments within the bulk primer coating for NPE-ZRP coating. Moreover, validation of the laboratory results with the extended outdoor exposures showed no detrimental effect of the replacement of nano-particles with conventional zinc content in NPE-ZRP coating.

- Pre-exposure of a prepared steel substrate to moisture content before the repair coating application didn't show a significant detrimental effect on coating Durability. Salt contamination, as well as rust formation and remnant coating layer from inappropriate surface cleaning of previously coating surface, showed to degrade the coating bond strength as well as durability.

\section{RECOMMENDATIONS FOR FUTURE RESEARCH}

In this research, the effectiveness and protection provided by the NPE-ZRP coating system have been identified but there would benefit from future research to improve the system and make it more durable for long service life. Based on the research finding the following recommendation is proposed. 
- Further research should compare the different percentages of zinc/nano-particle ratio to identify the optimum amount that can contribute to the long-term galvanic protection and can reduce the negative effect of the formation of the galvanic cell between the steel substrate and nano-particles.

- Research to develop a procedure that can help the zinc pigments to remain active for a longer time to provide enhanced cathodic protection. That will overcome the problem of losing the conductivity of zinc pigments due to the formation of zinc oxide around the outer surface with consumption.

- An in-depth exploration of how the nano-particles are contributing to the coating cohesive bond within the bulk primer layer and their interaction with the dispersed zinc pigments as well to the adhesive bond with the steel substrate would be very helpful to improve the coating durability. 


\section{REFERENCES}

Abreu, C.M., Izquierdo, M., Merino, P., Nóvoa, X.R. \& Pérez, C. (1999). A New Approach to the Determination of the Cathodic Protection Period in Zinc-Rich Paints. Corrosion Journal, 55(12), 1173-1181.

Alblas, B.P., \& Londen, A.M., (1997). The effect of chloride contamination on the corrosion of steel surfaces: a literature review. Protective Coating. Europe. 2(2), 16-25.

American Galvanizers Association. Duplex Systems-Painting over Hot-Dip Galvanized Steel. 2012. The HDG Coating. Retrieved on May 13, 2017, from https://www.galvanizeit.org/uploads/publications/Paint_Over_Galvanized_Steel_Duplex Systems.pdf

American Galvanizers Association. The HDG Coating. Retrieved on May 15, 2017, from https://www.galvanizeit.org/hot-dip-galvanizing/what-is-hot-dip-galvanizing-hdg/thehdg-coating

American Galvanizers Association. Corrosion Rate. Retrieved on May 15, 2019, from https://galvanizeit.org/corrosion/corrosion-process/corrosion-rate

Amo, B. D., Romagnoli, R., Deyá, C., \& González, J.A. (2002). High performance waterbased paints with non-toxic anticorrosive pigments. Progress in Organic Coatings, 45, 389-397.

Appleman, B. R. (1987). Painting Over Soluble Salts: A Perspective. Paint Square. Retrieved on May 4, 2017, from http://www.paintsquare.com/library/articles/SSPCBiblio2.PDF

Arronche, L., Gordon, K., Ryu, D., La Saponara, V., \& Cheng, L. (2013). Investigation of galvanic corrosion between AISI 1018 carbon steel and CFRPs modified with multiwalled carbon nanotubes. Journal of Materials Science. 48(3), 1315-1323.

DOI: $10.1007 / \mathrm{s} 10853-012-6876-2$

ASTM B 117-16. (2016). Standard Practice for Operating Salt Spray (Fog) Apparatus. ASTM Book of Standards.

ASTM D 4541-02. (2002). Standard Test Method for Pull-Off Strength of Coatings Using Portable Adhesion Testers. ASTM Book of Standards.

ASTM G 7M-11. (2011). Standard practice for atmospheric environmental exposure testing of nonmetallic materials. ASTM Book of Standards.

Axelsen, S.B. \& Knudsen, O.Ø. (2011). The effect of water-soluble salt contamination on coating performance. NACE, CORROSION Conference. (Paper- 11042) 
Barsoukov, E., \& Macdonald, J. R. (2005). Impedance spectroscopy: theory, experiment, and applications ( $2^{\text {nd }}$ ed.). Hoboken: Wiley Inter Science.

Bayer, G. T. \& Zamanzadeh, M. (2004). Failure Analysis of Paints and Coatings. Published Internally by Matco Associates.

Bernecki, T., Clement, K., Cox, E. Kogler, R., Lovelance, C., Peart, J., \& Verma, I. (1997). Bridge maintenance coatings. FHWA Study Tour for Bridge Maintenance Coatings.

Bierwagen, G. P. (1996). Reflections on corrosion control by organic coatings. Progress in Organic Coatings, 28 (1), 43-48.

Broomfield, J. P. (1997). Corrosion of Steel in Concrete: Understanding, Investigation, and Repair, E \& FN Spon, UK, 240.

Biezma1, M. V. \& Schanack, F. (2007). Collapse of Steel Bridges, / Journal of Performance of Constructed Facilities, Vol. 21(5), p. 398-405.

Cabanelas, I., Collazo, A., Izquierdo, M., No’voa, X.R. \& Pe'rez C. (2007). Influence of galvanised surface state on the duplex systems behavior. Corrosion Science, Vol. 49 (4), 1816-1832.

Castro, P., Sagüés, A. A., Moreno, E. I., Maldonado, L., \& Genescá, J. (1992). Characterization of activated titanium solid reference electrodes for corrosion testing of steel in concrete. Corrosion Science, 52 (8), 609-617.

Catbas, F. N., Malekzadeh, M. \& Khuc, T. (2013). Movable Bridge Maintenance Monitoring. Florida Department of Transportation. (Project No. BDK78-977-10).

Cederquist, S.C. (2000). Motor Speedway Bridge Collapse Caused by Corrosion. Materials performance Vol. 39(7), p.18-19.

Chang \& Chung (1999). Steel bridge protection policy, Indiana Department of Transportation and Purdue University, 2. (Report No. FHWA/IN/JTRP-98/21)

Chang, L.M. \& Georgy, M. (1999). Metallization of steel bridges: research and practice. Indiana DOT and Purdue University. (Report No. FHWA/IN/JTRP-98/21)

Chang, L., Zayed, T., \& Fricker, J.D. (1999). Steel bridge protection policy: Evaluation of bridge coating system for INDOT steel bridges. Indiana Department of Transportation and Purdue University. (Report No. FHWA/IN/JTRP-98/21-II) 256 
Chong, S.L. \& Yao, Y. (2007). Selecting Overcoats for Bridges. Federal Highway

Administration, Vol. 71(2). (Publication Number: FHWA-HRT-07-006).

Containing Carbon Nanotubes on Carbon Steel in Simulated Concrete Pore Environments. Corrosion, 72 (11), 1397-1423.

Cubides, Y. \& Castaneda, H. (2016). Corrosion Protection Mechanisms of Carbon Nanotube and Zinc-Rich Epoxy Primers on Carbon Steel in Simulated Concrete Pore Solutions in the Presence of Chloride Ions. Corrosion Science, 109, 145-161.

Cubides, Y., Su, S. S. \& Castaneda, H. (2016). Influence of Zinc Content and Chloride Concentration on the Corrosion Protection Performance of Zinc-Rich Epoxy Coatings

Dehri, I. \& Erbil, M. (2000). The effect of relative humidity on the atmospheric corrosion of defective organic coating materials: an EIS study with a new approach. Corrosion Science, 42, 969-978.

Dmitruk, A., Mayer, P., \& Pach, J. (2018). Pull-off strength of thermoplastic fiberreinforced composite coatings, Journal of Adhesion Science and Technology. 32 (9), 997-1006.

Drozdz, S. (2012). Carbon Nanotube-Infused Zinc Coating Combines Barrier Properties with Cathodic Protection. U.S. Army Engineer Research and Development Center.Construction Engineering Research Laboratory, Retrieved on February 3, 2016, from http://www.erdc.usace.army.mil/Media/NewsStories/tabid/9219/Article/476175/carbonnanotube-infused-zinc-coating-combines-barrier-properties-with-cathodic.aspx

Dudley, J. (2003). Specifications and Polyurea Elastomeric Coating/Lining Systems. PDA Annual Conference, Reno, Nevada.

El-Mahdy, G. A., Nishikata, A., \& Tsuru, T. (2000). AC impedance study on corrosion of 55\% Al-Zn alloy-coated steel under thin electrolyte layers. Corrosion Science. 42(9), 1509-1521.

Fancy, S. F., Sabbir, M. A., Lau, K., \& DeFord, D. (2017). Corrosion Performance of Nano-Particle Enriched Epoxy Primer for Marine Highway Bridge Application. CORROSION/17, New Orleans, LA. (Paper No. 9539)

Federal Highway Administration. (1995). Bridge Coatings Technical Note. Zinc-Rich Bridge Coatings. Retrieved on May 8, 2017, from https://www.fhwa.dot.gov/publications/research/infrastructure/structures/bridge/zinc.cfm

Federal Highway Administration. (2015). Steel Bridge Design Handbook. Corrosion Protection of Steel Bridges, 19. (Publication No. FHWA-HIF-16-002). 
Florida Department of Transportation, Bridge Environment Data Retrieved on January 5, 2017 from

http://www.fdot.gov/materials/administration/resources/library/publications/bridge/enviro nment.pdf

Florida Department of Transportation. (2018). Standard Specifications for Road and Bridge Construction, Section 975.

Fontana, M., \& Greene, N. (1986). Corrosion Engineering (3rd ed.): McGraw Hill.

Francis, R., (2016). What Do You Know about Inorganic Zinc Coatings? Materials Performance. Retrieved on January 5, 2018, from http://www.materialsperformance.com/articles/coating-linings/2016/06/what-do-you-knowabout-inorganic-zinc-coatings

Fuente, D. de la., Chico, B. \& Morcillo, M. (2006), The Effects of Soluble Salts at the Metal/ Paint Interface: Advances in Knowledge. Portugaliae Electrochimica Acta, 24, 191-206.

Fukuda, H., Kondoh, k., Umeda, J., \& Fugetsu, B. (2011). Fabrication of magnesium based composites reinforced with carbon nanotubes having superior mechanical properties. Materials Chemistry and Physics. 127(3), 451-458.

DOI:10.1016/j.matchemphys.2011.02.036

Gergelya, A., Pászti, Z., Mihályc, J., Drotár E. \& Töröka, T. (2014). Galvanic function of zinc-rich coatings facilitated by percolating structure of the carbon nanotubes. Part II:

Protection properties and mechanism of the hybrid coatings. Progress in Organic Coatings, 77, 412-424.

Hammouda, N., Chadli, H., Guillemot, G. \& Belmokre, K. (2011). The Corrosion Protection Behaviour of Zinc Rich Epoxy Paint in 3\% NaCl Solution. Advances in Chemical Engineering and Science, 1, 51-60.

Hare, C. H. (1984). Non-Osmotically-Induced Blistering Phenomena on Metal. Journal of Protective Coatings \& Linings: The voice of the Steel Structures Painting Council. Pittsburgh, Pa: Technology Publ. Co.

Hare, C. H. (1989). Barrier Coatings. Journal of Protective Coatings \& Linings, 6 (2), 5969.

Helsel, J. L. (2015). Problems with Galvanizing. The Journal of Protective Coatings \& Linings. Retrieved on January 23, 2016, from http://www.paintsquare.com/archive/?fuseaction=view\&articleid=5658 
Hsu, C. H., \& Mansfeld, F. Technical Note: Concerning the conversion of the constant phase element parameter Y0 into a capacitance. Corrosion Science, 57 (9), 747-748.

Iijima, S. (1991) “Helical microtubules of graphitic carbon”, Nature, 354, 56.

Jeffery, L. (2009). Fall of the Eighth Wonder: The Kinzua Bridge. Retrieved at January 23, 2019, from https://pabook.libraries.psu.edu/literary-cultural-heritage-map-pa/featurearticles/fall-eighth-wonder-kinzua-bridge

Johari, N., Hassan, N. A., Hassan, N., \& Ani, M. H. (2016). Effect of Nanoparticles on Wettability of Nanocoating on Carbon Steel. International Journal of Engineering Materials and Manufacture. 1(2), 71-74.

Jones, D. A. (1996). Principle and preservation of corrosion. (2nd ed.): Prentice Hall Kodumuri, P. \& Lee, S. K. (2012). Federal Highway Administration 100-Year Coating Study. Federal Highway Administration. (Repost No. FHWA-HRT-12-044).

Khalid, M., Naser, M. A. \& Joe, B. (2018). Investigation, Design, and Repair of a Major Bridge Foundation Failure: I-43 Leo Frigo Memorial Bridge.Transportation Research Board 97th Annual Meeting, Washington DC, United States.

Kline, E. (2009). Durable Bridge Coatings. Modern Steel Construction.

Kodumuri, P. \& Lee, S. K. (2012). Federal Highway Administration 100-Year Coating Study. Federal Highway Administration. (Report No. FHWA-HRT-12-044).

Koger, R.A., Brydl, D., \& Highsmith, C. (1998). Recent FHWA experience in testing and implementing metallized coatings for steel bridges. Corrosion-98. NACE Int. Houston, TX. (Paper No. 499)

Kogler Jr, R. A. \& Chong, S.L. (1997). Steel Bridge Coatings Research. Public Roads, 61(1).

Kolek, Z. (1997). Characterization of Water Penetration inside Organic Coatings by Capacitance Measurements. Progress in Organic Coating, 30(4), 287-292.

LeBozec, N., Persson, D., Thierry, D. \& Axelsen, S.B. (2004). Effect of Climatic Parameters on Filiform Corrosion of Coated Aluminum Alloys. Corrosion Journal, Corrosion Engineering Section, 60 (6), 584-593.

Li, X., \& Castaneda, H. (2018). Application of Electrochemical Techniques on Study of Effect of Nano-ZnO in Conductive Polyaniline Containing Zinc-Rich Primer.

International Journal of Spectroscopy, 2018, 15. https://doi.org/10.1155/2018/7160381.

Liu W. M. (1998). Efficiency of barrier-effect and inhibitive anti-corrosion pigments in primers. Materials and Corrosion, 49, 576-584. 
Mahdavian, M., \& Attar, M. M. (2006). Another approach in analysis of paint coatings with eis measurement: phase angle at high frequencies. Corrosion Science, 48(12), 41524157.

Materials Performance (2002, July). Corrosion Costs and Preventive Strategies in the United States. NACE International, Houston, TX.

Mayne, J. E. O. (1959). “The problem of painting rusty steel.” Journal of Applied Chemistry 9, 673-680. 257.

McCafferty, E. (2010). Introduction to corrosion science. Springer New York Dordrecht Heidelberg London, 127.

Meade, B.W., Palle, S., Hopwood II, T. \& Younce, Rick. (2010). Effect of Chloride Contamination on Coating Performance. Research Report, Kentucky Transportation Center. (KTC-10-10/SPR 366-08-1F).

Mittal, K. L. (1983). Adhesion Aspects of Polymeric Coatings. New York: Plenum Press.

Morcillo, M. (2003). Soluble salts: their incidence on the protection of metallic structures by paint coatings. Rev. Metal. Madrid, Extr. 219-227.

Morrison, J. D. (1980). Report on the Relative Corrosivity of Atmospheres at Various Distances from the Seacoast, NASA-Kennedy Space Center. (Report No. MTB 099-74)

Munger, C. G. \& Vincent, L. D. (2014). Corrosion Prevention by Protective Coatings. Edition 3, NACE International. (ISBN 1575902621, 9781575902623).

Murray, J. N. (1997). Electrochemical test methods for evaluating organic coatings on metals: An update. part iii: multiple test parameter measurements. Progress in Organic Coating, 31 (4), 375-391.

Myers, J. J., Zheng, W., \& Washer, G. (2010). Structural steel coatings for corrosion mitigation. Missouri Department of Transportation. (Report No. OR11.006)

National Atmospheric Deposition Program /National Trends Network, (2015), Chloride Ion Concentration and Wet Deposition. Retrieved on April 9, 2017, from http://nadp.sws.uiuc.edu/maplib/pdf/2015/Cl_conc_2015.pdf and http://nadp.sws.uiuc.edu/maplib/pdf/2015/Cl_dep_2015.pdf

National physical Laboratory. (2015). Corrosion protection of steel bridges. Online corrosion guide, Corus Construction \& Industrial, Retrieved on January 8, 2015, from http://resource.npl.co.uk/docs/science technology/materials/life_management_ofmaterial s/publications/online_guides/pdf/protection_of_steel_bridges 
NCHRP, (2007). Guidelines for the Selection of Snow and Ice Control Materials to Mitigate Environmental Impacts. National Cooperative Highway Research Program, Transportation Research Board, Washington, D. C. (Report No. 557)

NTSB (1983). Collapse of Suspended Span of Route 95 Highway Bridge over the Mianus River, Greenwich, Connecticut, highway accident report. Retrieved on April 9, 2019, from https://eng-resources.uncc.edu/failurecasestudies/bridge-failure-cases/mianus-riverbridge/

Park, S. M. \& Shon, M. Y. (2015). Effects of multi-walled carbon nano tubes on corrosion protection of zinc rich epoxy resin coating. Journal of Industrial and Engineering Chemistry, 21, 1258-1264.

Phifer, E.M. (1993). Evaluation of experimental painting of A588 steel bridges. Michigan Department of Transportation. (Report No. R-1324).

Porter, F. C., (1994). Corrosion Resistance of Zinc and Zinc Alloys. Marcel Dekker Inc., New York, 334.

Pouliotte, J. (2012). FDOT New Directions in Steel Corrosion Protection, Presentation.

Prasanna, R. (2016). Effect of Humidity on Surface Preparation and Coating Application. Coatings and Linings, Materials Performance. Retrieved on May 2017, from http://www.materialsperformance.com/articles/coating-linings/2016/02/effect-ofhumidity-on-surface-preparation-and-coating-application

Praveen, B.M., Venkatesha, T.V., Naik, Y. A. and Prashantha, K. (2007). Corrosion Studies of Carbon Nanotubes-Zn Composite Coating. Surface \& Coatings Technology. 201, 5836-5842.

Prosek, T. \& Thierry, D. (2004). A Model for the Release of Chromate from Organic Coatings. Progress in Organic Coatings, 49, 209-21.

Rashidi N, Alavi-Soltani S, \& Asmatulu R. (2007). Crevice corrosion theory, mechanisms and prevention methods. Proceedings of the 3rd Annual GRASP Symposium, Wichita State University, 215-216.

Rudawska, A., \& Kuczmaszewski, J. (2006). Surface free energy of zinc coating after finishing treatment. Materials Science-Poland. 24(4), 975-981.

Sabbir, M. A. (2017). Corrosion Degradation Mechanism of CBPC Coating System for Highway Bridge Steel Components", Ph.D. Dissertation. DOI:

10.25148/etd.FIDC001797. 
Schaefer, K. \& Miszczyk, A. (2013). Improvement of electrochemical action of zinc-rich paints by addition of Nano particulate zinc. Corrosion Science, 66, 380-391.

Seneviratne, A.M.G., Sergi, G., \& Page, C.L. (2000). Performance characteristics of surface coatings applied to concrete for control of reinforcement corrosion. Construction and Building Materials, 14(01), 55-59.

Shreepathi, S., Bajaj, P. \& Mallika, B.P. (2010). Electrochemical Impedance Spectroscopy Investigations of Epoxy Zinc Rich Coatings: Role of Zn Content on Corrosion Protection Mechanism. Electrochemical Acta, 55(18), 5129-5134.

Sørensen, P. A., Kiil, S., Dam-Johansen, K. \& Weinel, C. E. (2009). Anticorrosive coatings: a review. Journal of Coatings Technology and Research, 6 (2), 135-176.

SSPC-SP 10 Near-White Metal Blast Cleaning. SSPC Standard.

SSPC-SP 3 Power Tool Cleaning. SSPC Standard.

Su X.L, \& Zhang X.Y. (2004). Bonding strength of interface between the coating and the substrate and its measurement. Electroplating \& Pollution Control. 24, 6-11.

Syed, S. (2006). Atmospheric Corrosion of Materials. Emirates Journal for Engineering Research, 11(1), 1-24.

Tam, C. K. \& Stiemer, S. F. (1996). Development of Bridge Corrosion Cost Model for Coating Maintenance. Journal of Performance of Constructed Facilities, 10(2), 47-56.

Tesla Nanocoating. Retrieved on June 12, 2015, from http://www.teslanano.com/product/1101-primer/

Transportation Research Synthesis. (2014). Transportation Agency Practices Currently Employed for Bridge Maintenance Painting Operations: Findings from a National Survey, KTA-Tator, Inc.(TRS 1404)

Wise, M. E., Semeniuk, T. A., Bruintjes, R., Martin, S. T., Russell, L. M. \& Buseck, P. R. (2007). Hygroscopic behavior of $\mathrm{NaCl}$-bearing natural aerosol particles using environmental transmission electron microscopy. Journal of Geophysical Research, 112, 1-12.

Yeomans, S. R. (2004). Galvanized Steel Reinforcement in Concrete: An Overview. Elsevier Science. (ISBN: 0-08-044511-X).

Zhang, D., Qian H., Wang, L. \& Lia, X. (2016). Comparison of barrier properties for a super hydrophobic epoxy coating under different simulated corrosion environments. Corrosion Science, 103, 230-241. 
Zhang, Y., Bai, Y. \& Yan, B. (2010). Functionalized carbon nanotubes forpotential medicinal applications. Drug Discov Today, Vol. 15(11-12), p.428-35.

Zhmurkin, D. (2009). Corrosion resistance of bolt coatings. Technical Paper, Tyco Electronics.

Zierden, D. F. (2014). Humidity in Florida. Retrieved on April 3, 2017, from https://climatecenter.fsu.edu/images/docs/Humidity-in-Florida.pdf 
APPENDICES 
APPENDIX - A

Inland Exposure NPE-ZRP Unscribed Sample

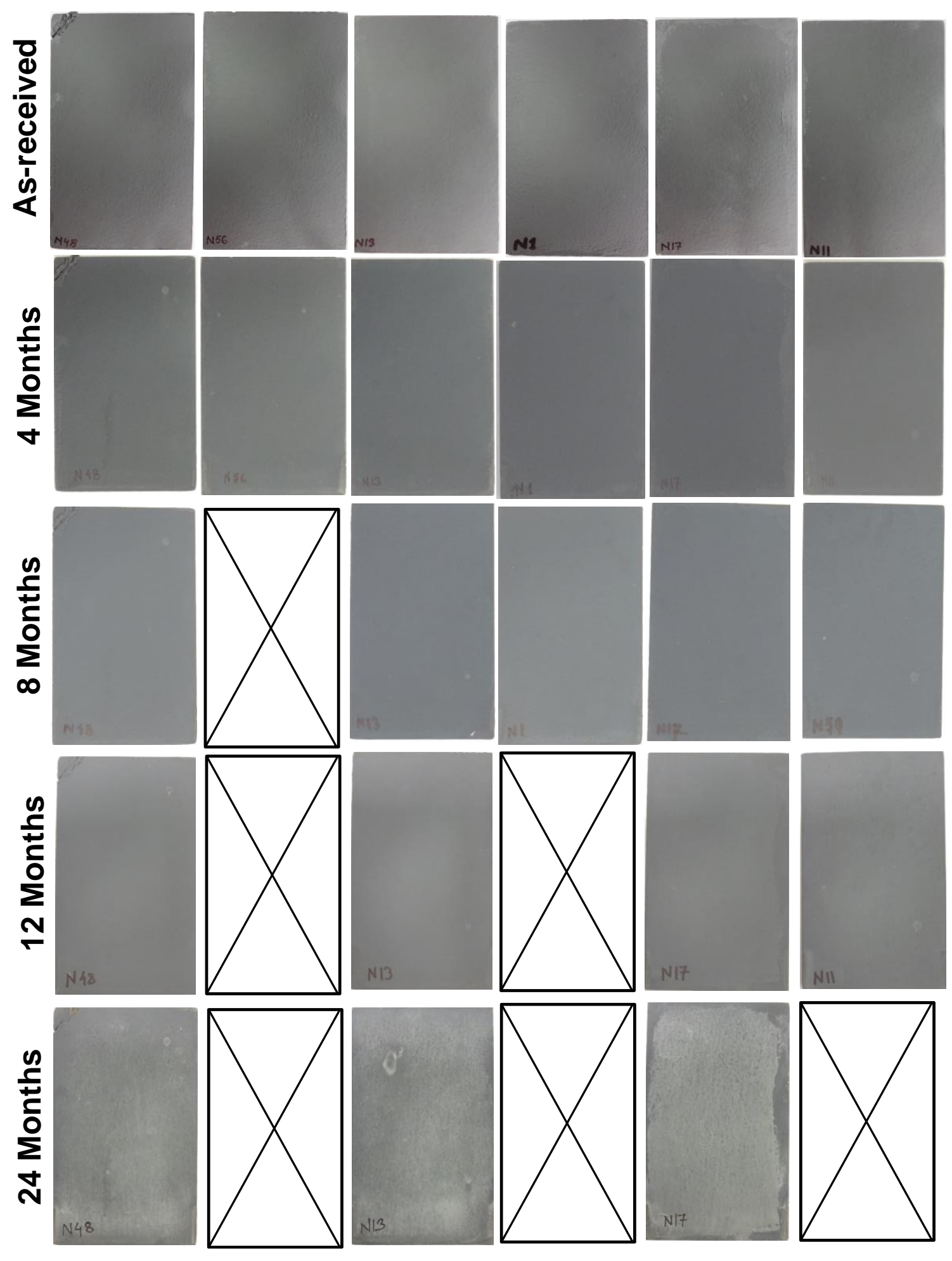


Inland Exposure NPE-ZRP Unscribed Sample
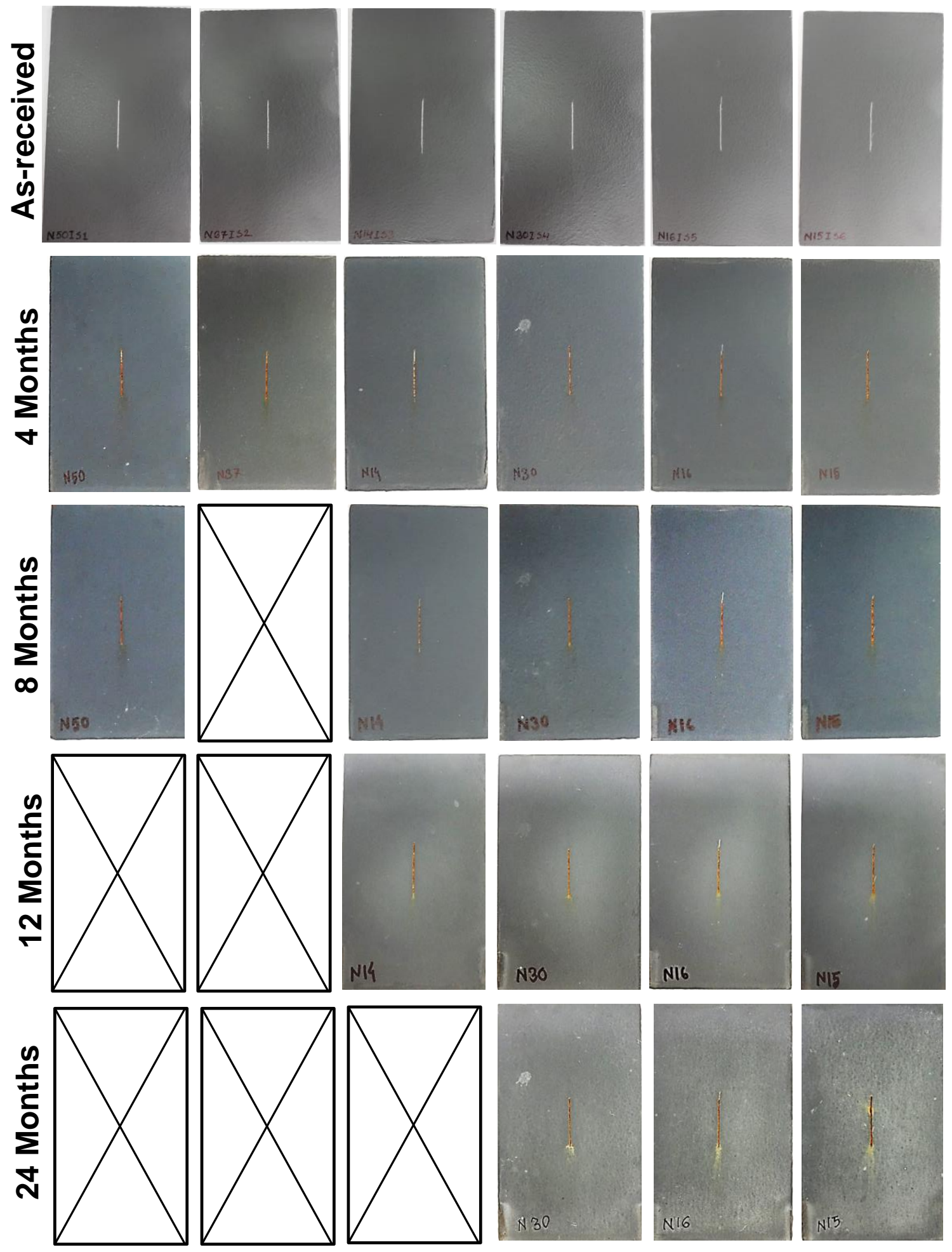
Beach Exposure NPE-ZRP Unscribed Sample

$\frac{8}{d}$
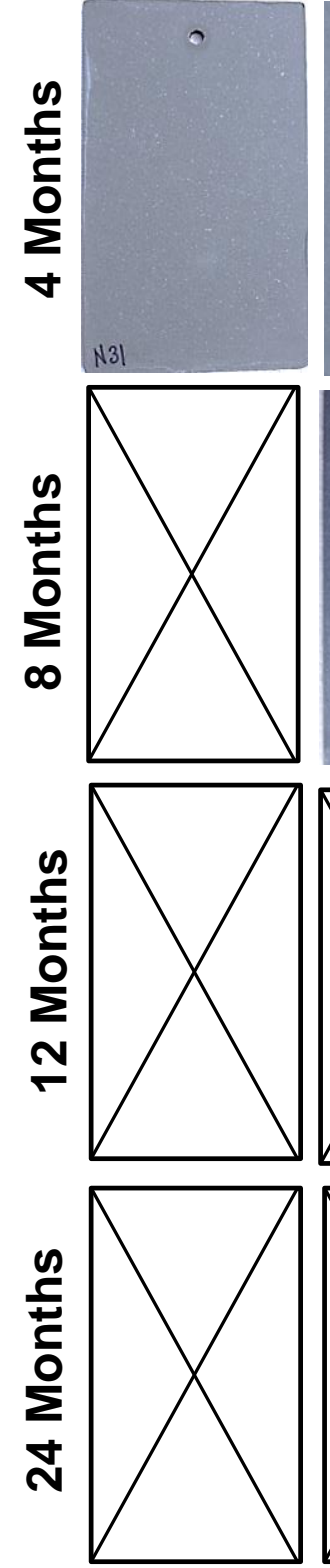
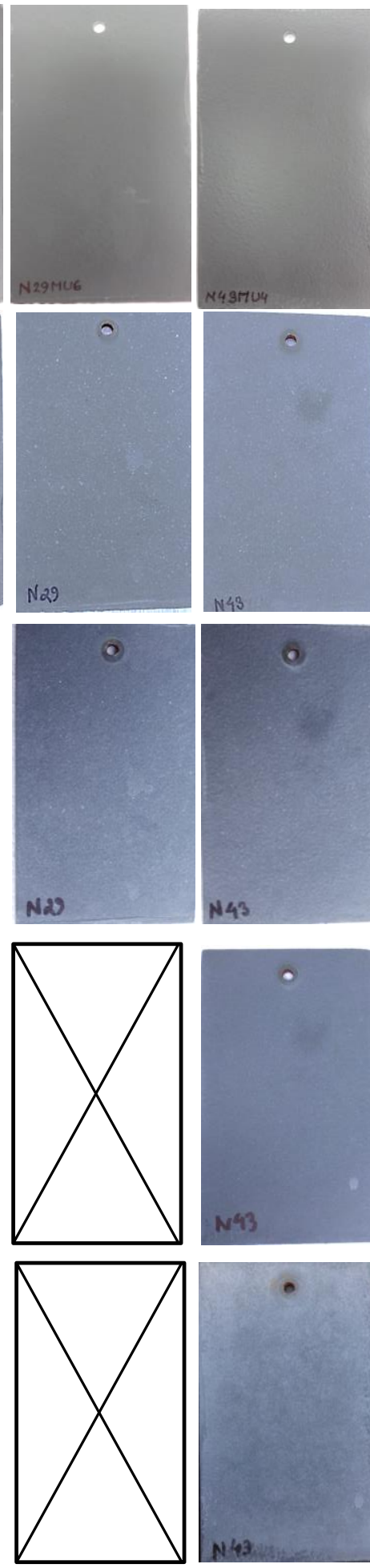
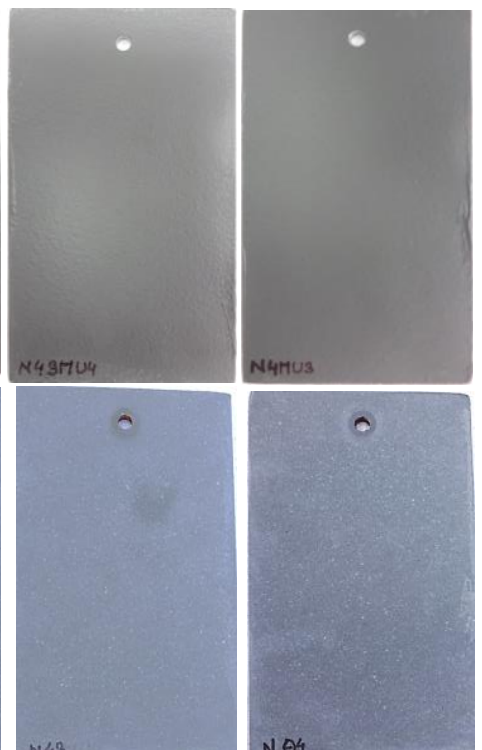

NO4
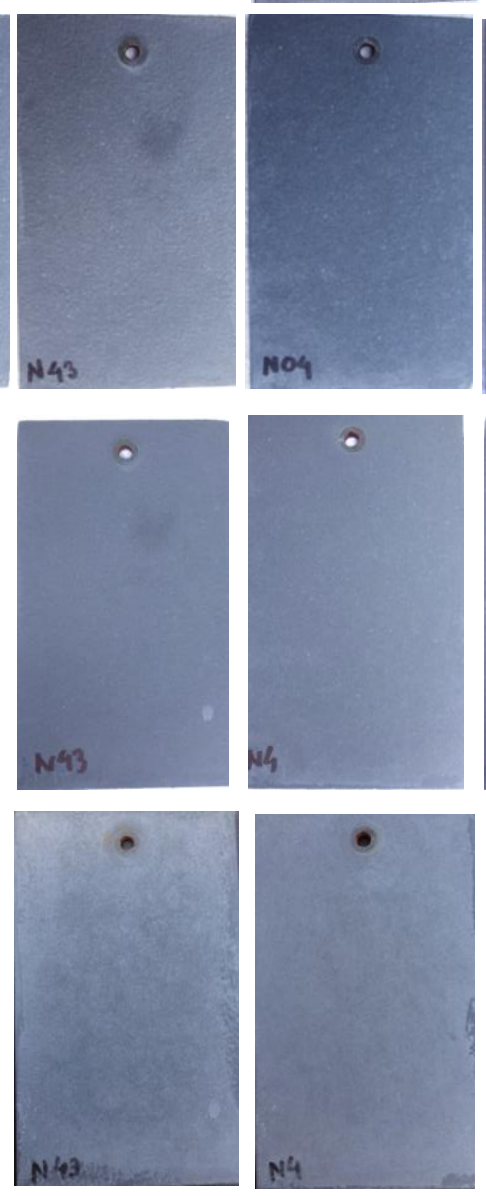
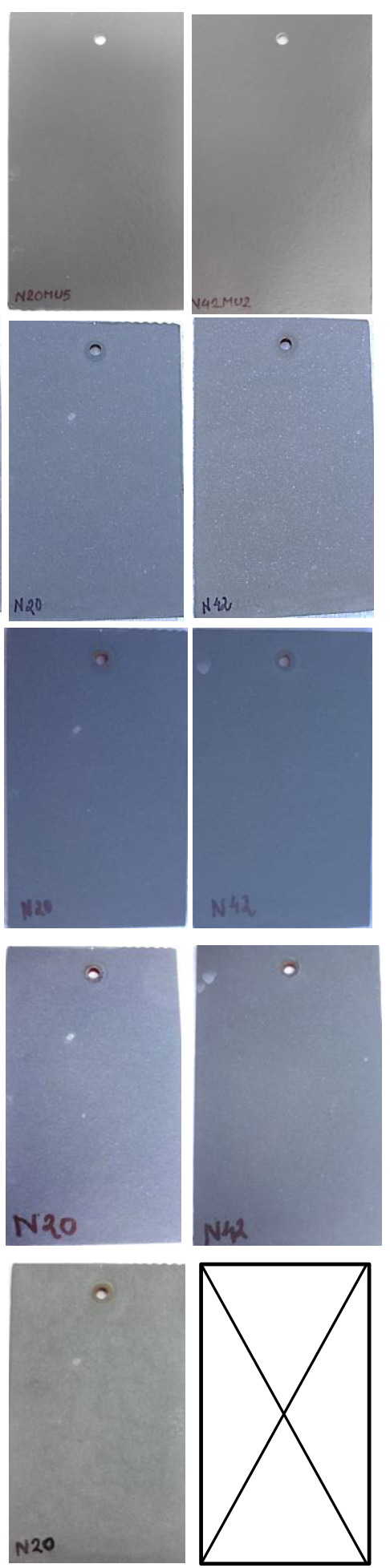
Beach Exposure NPE-ZRP Scribed Sample
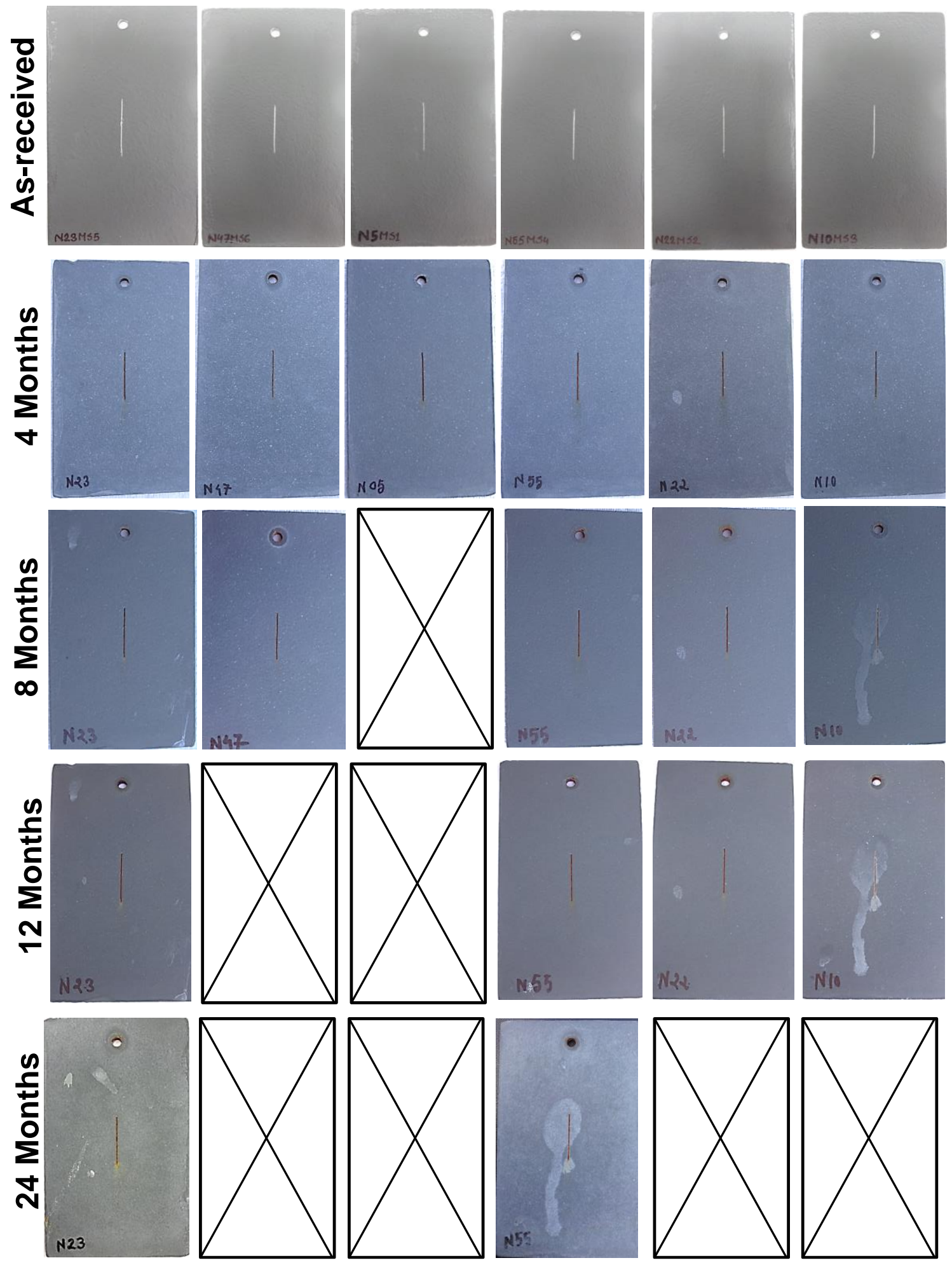
Salt-fog Exposure NPE-ZRP Unscribed Sample
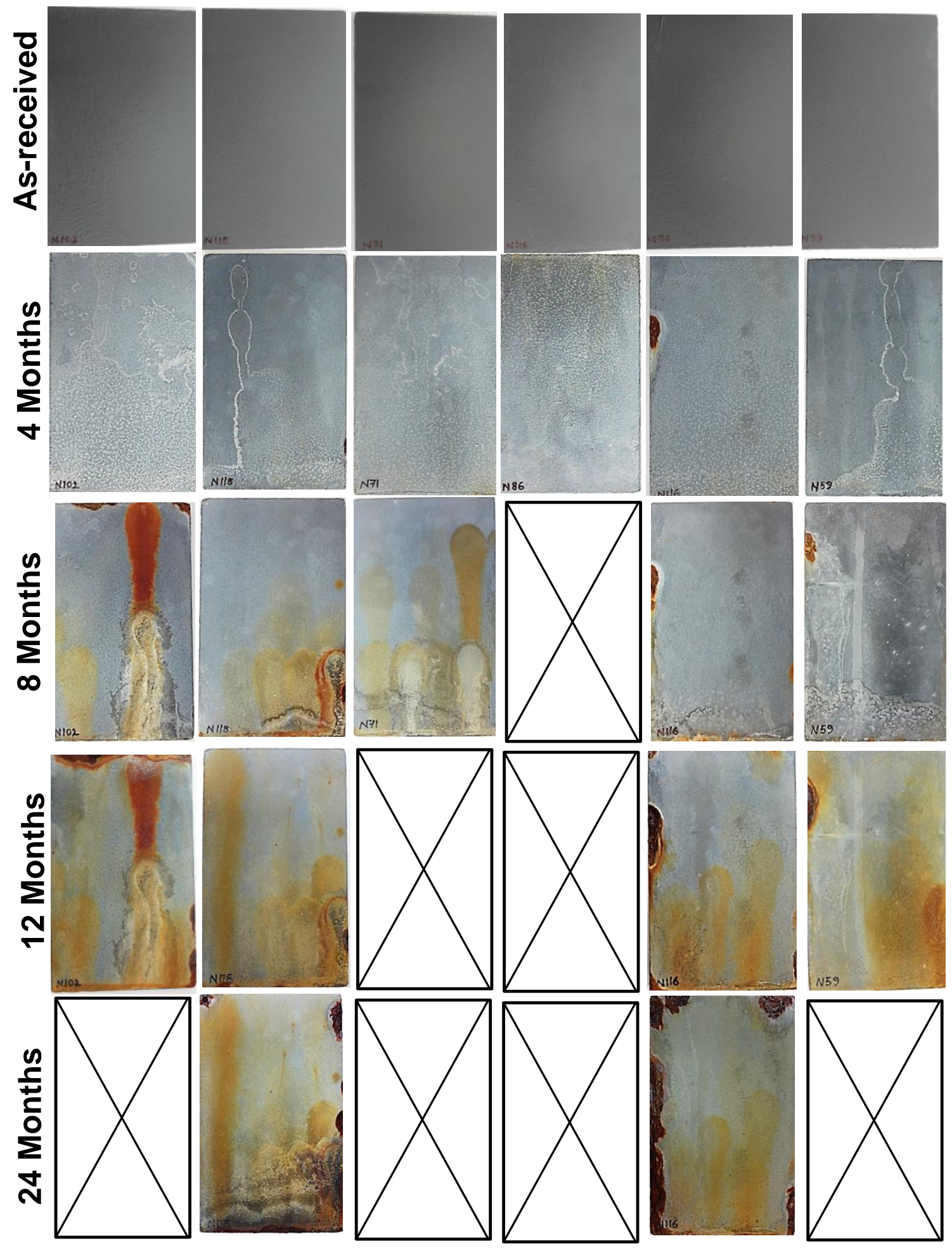
Salt-fog Exposure NPE-ZRP Scribed Sample
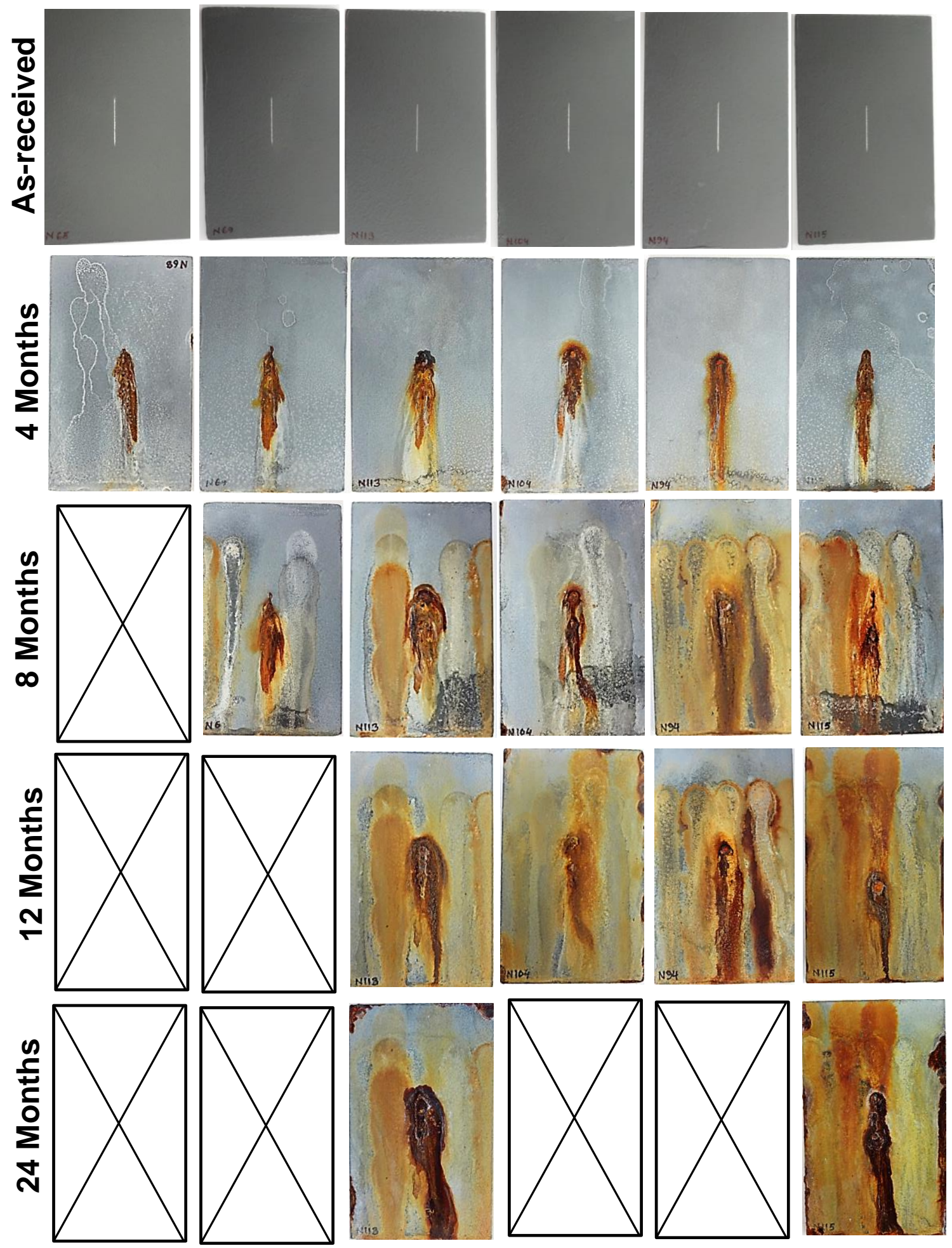
Inland Exposure ZRP Unscribed Sample

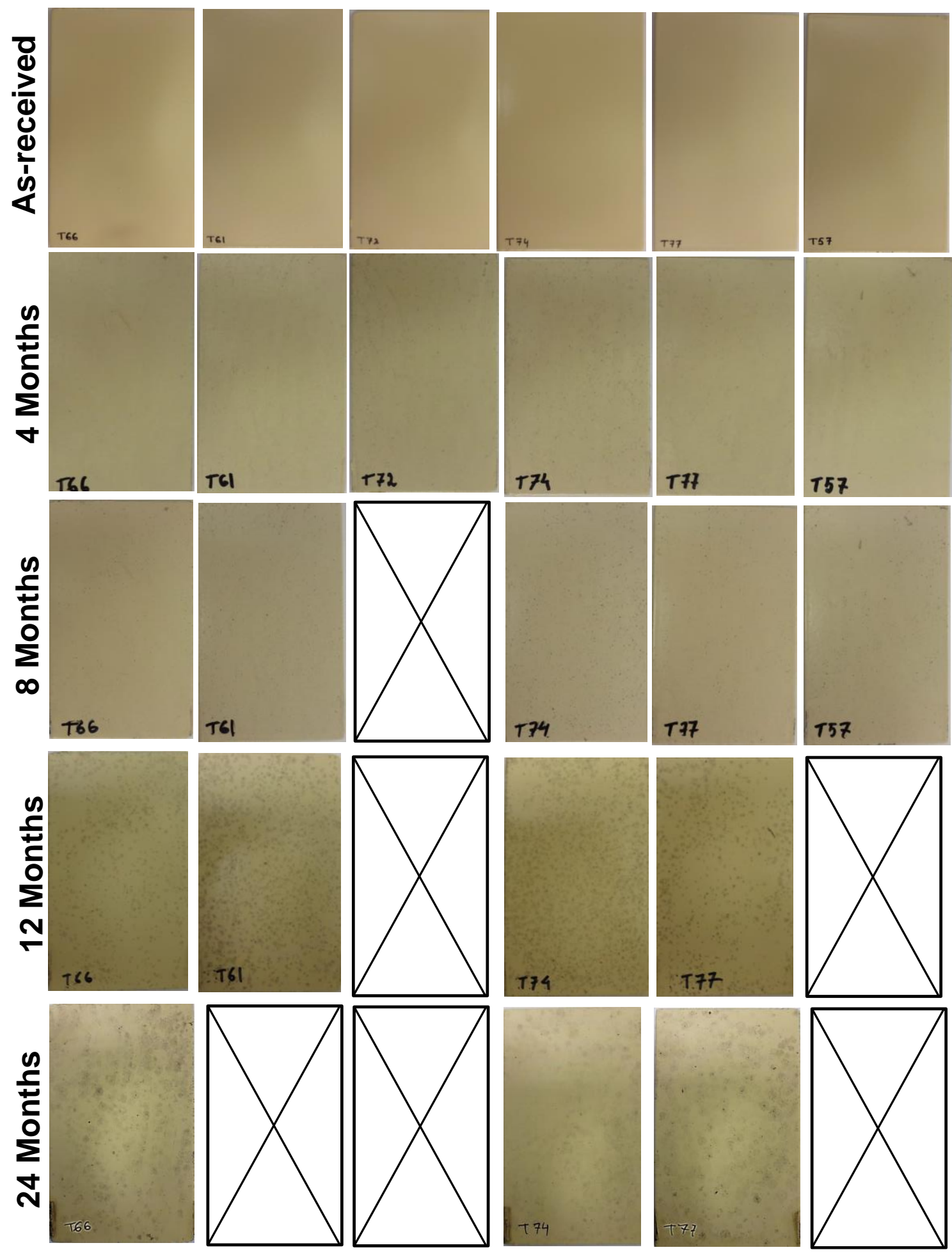


Inland Exposure ZRP Scribed Sample

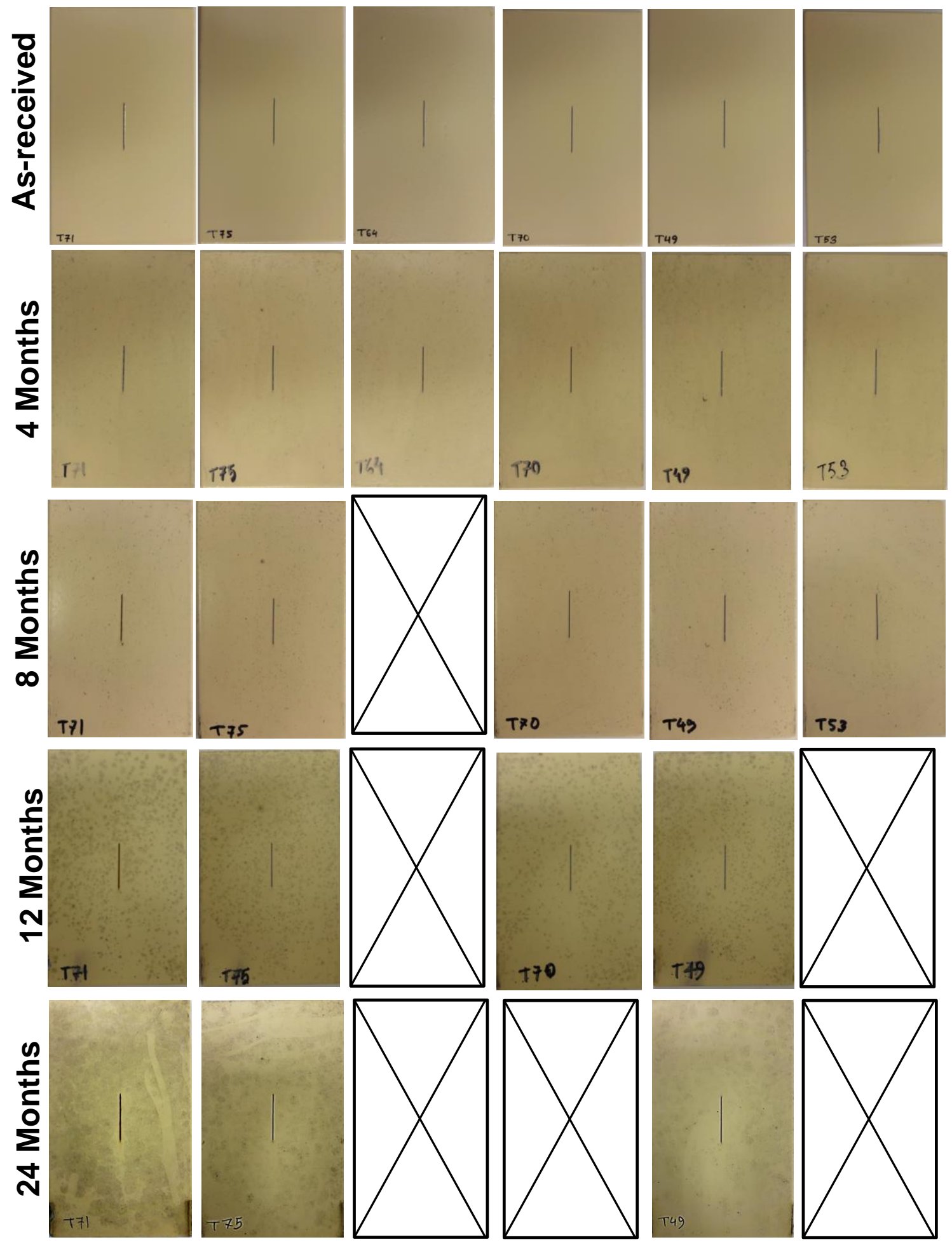


Beach Exposure ZRP Unscribed Sample
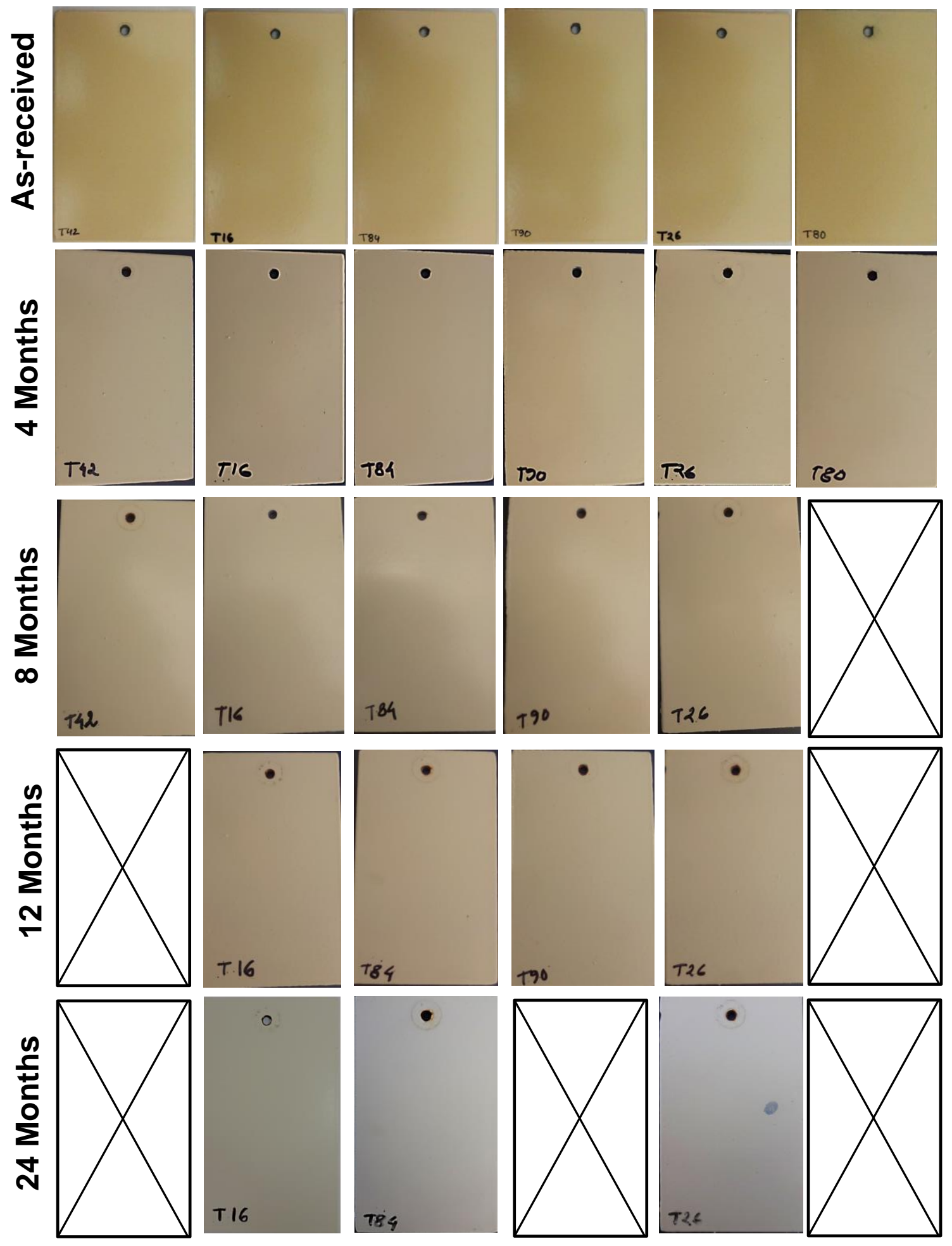
Beach Exposure ZRP Scribed Sample
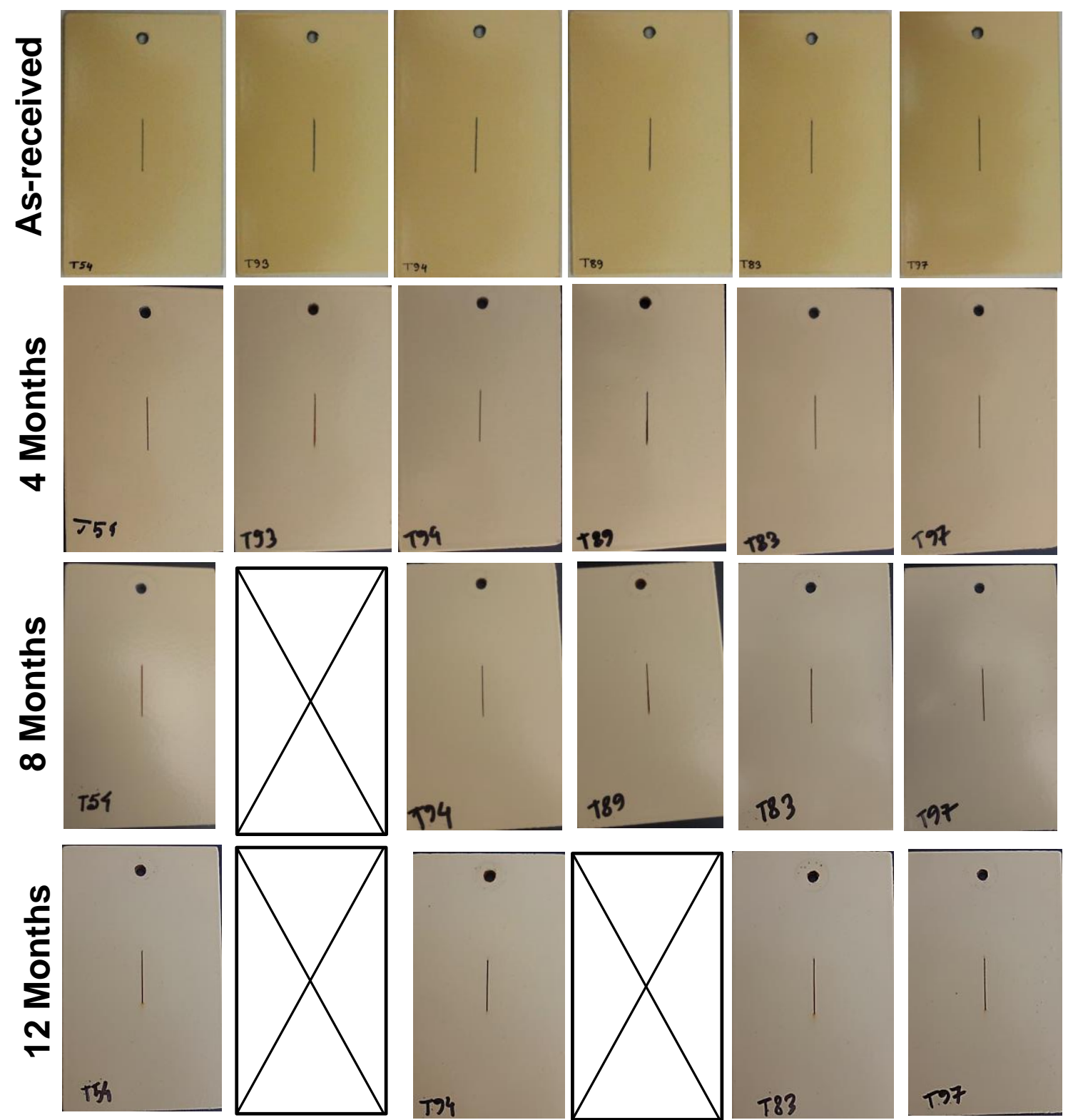

ז04
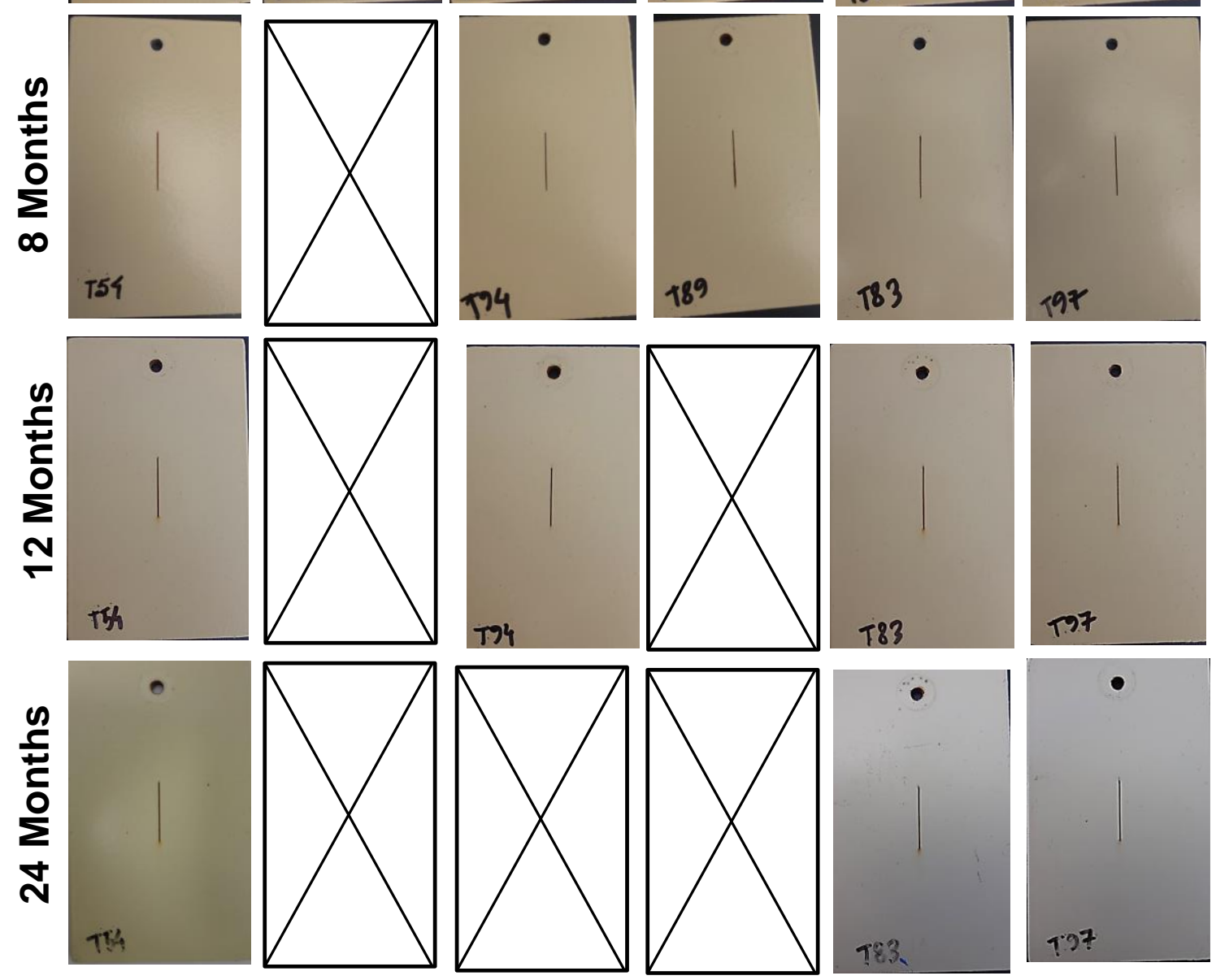
Salt-fog Exposure ZRP Unscribed Sample
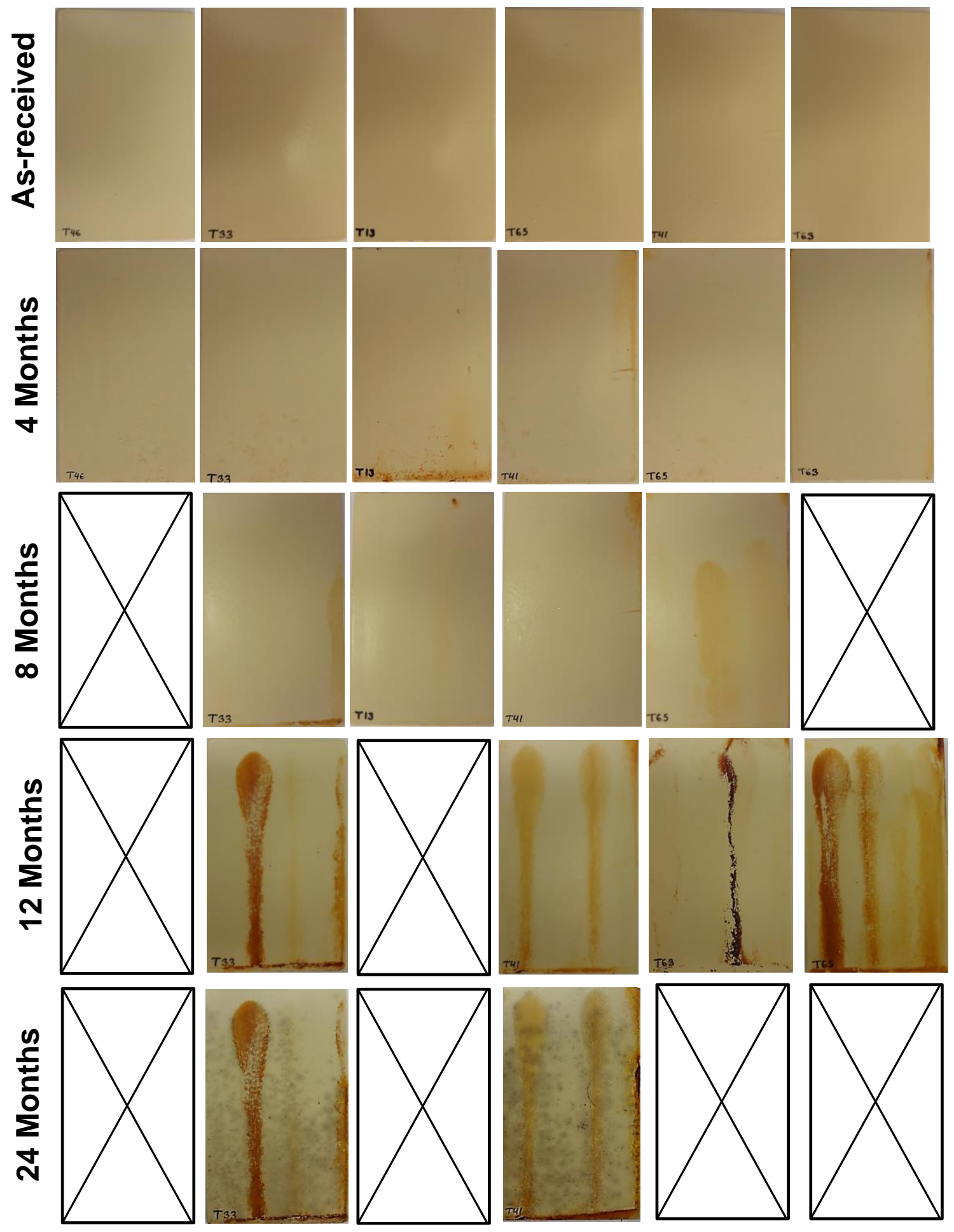
Salt-fog Exposure ZRP Scribed Sample
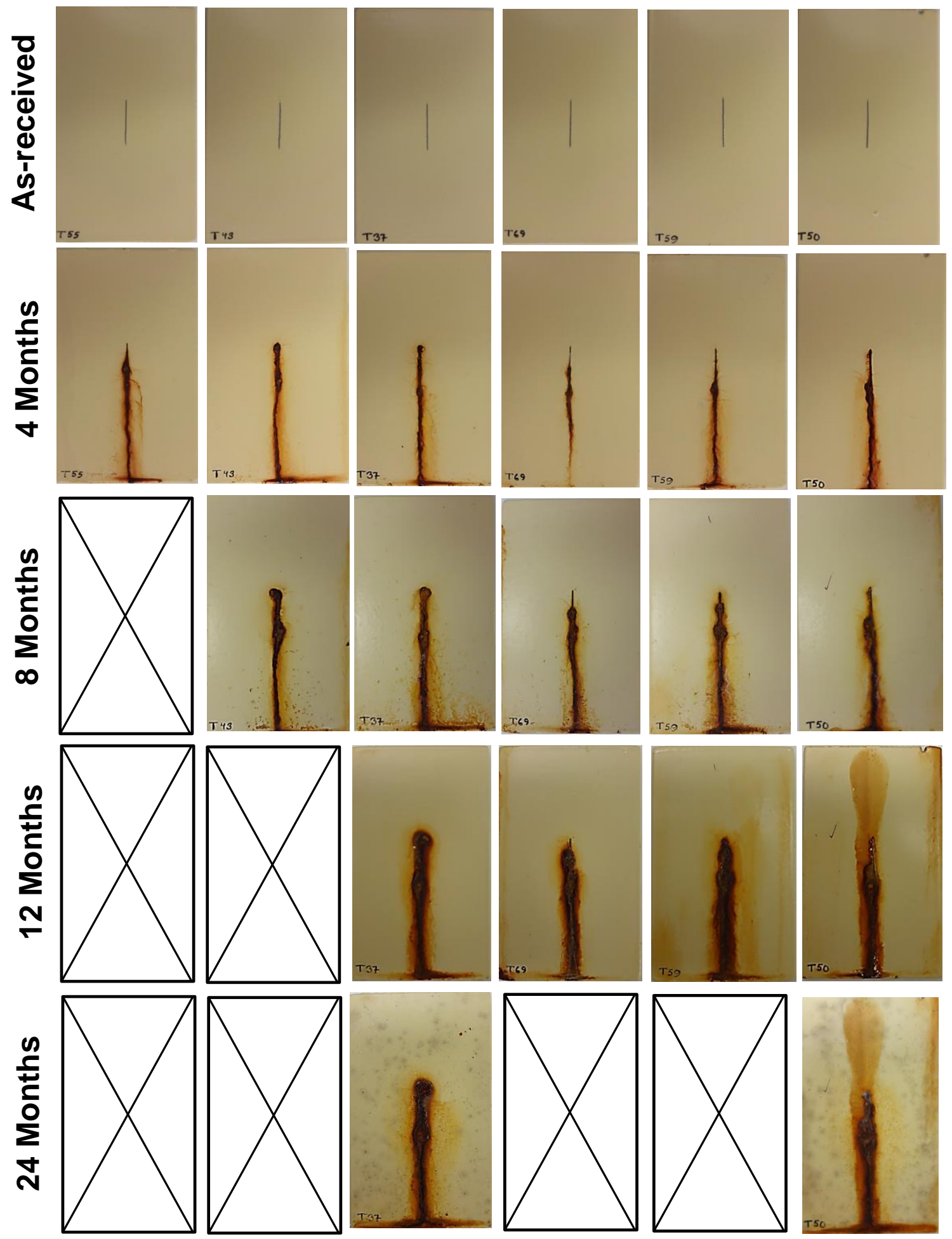


\section{APPENDIX - B}

Pull-off Strength Failure Modality of NPE-ZRP Samples
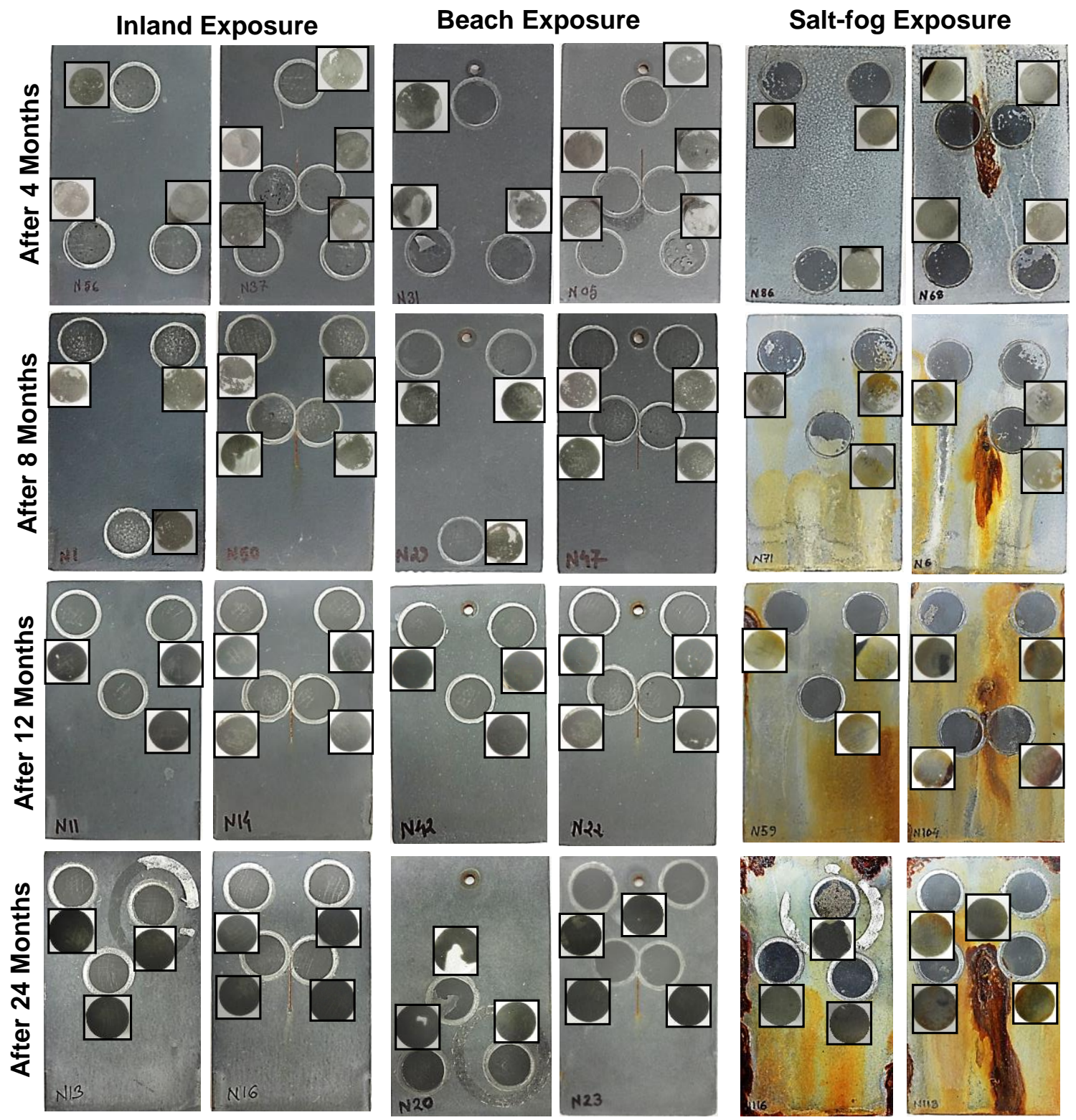


\section{Pull-off Strength Failure Modality of ZRP Samples}
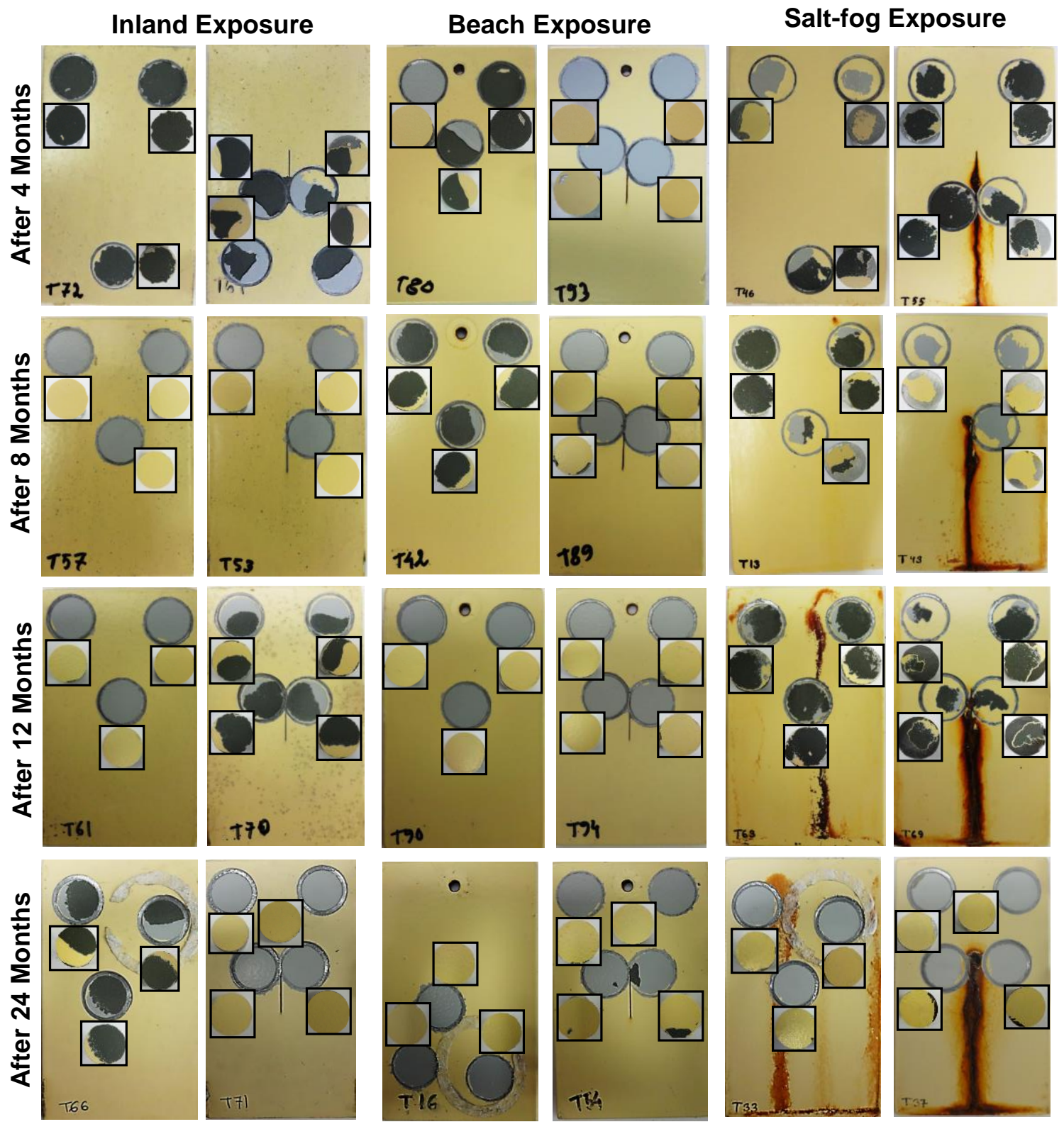
APPENDIX C- Exposed Repair Samples

Out-door Exposure NPE-ZRP Repaired Sample (Sand-blasted)
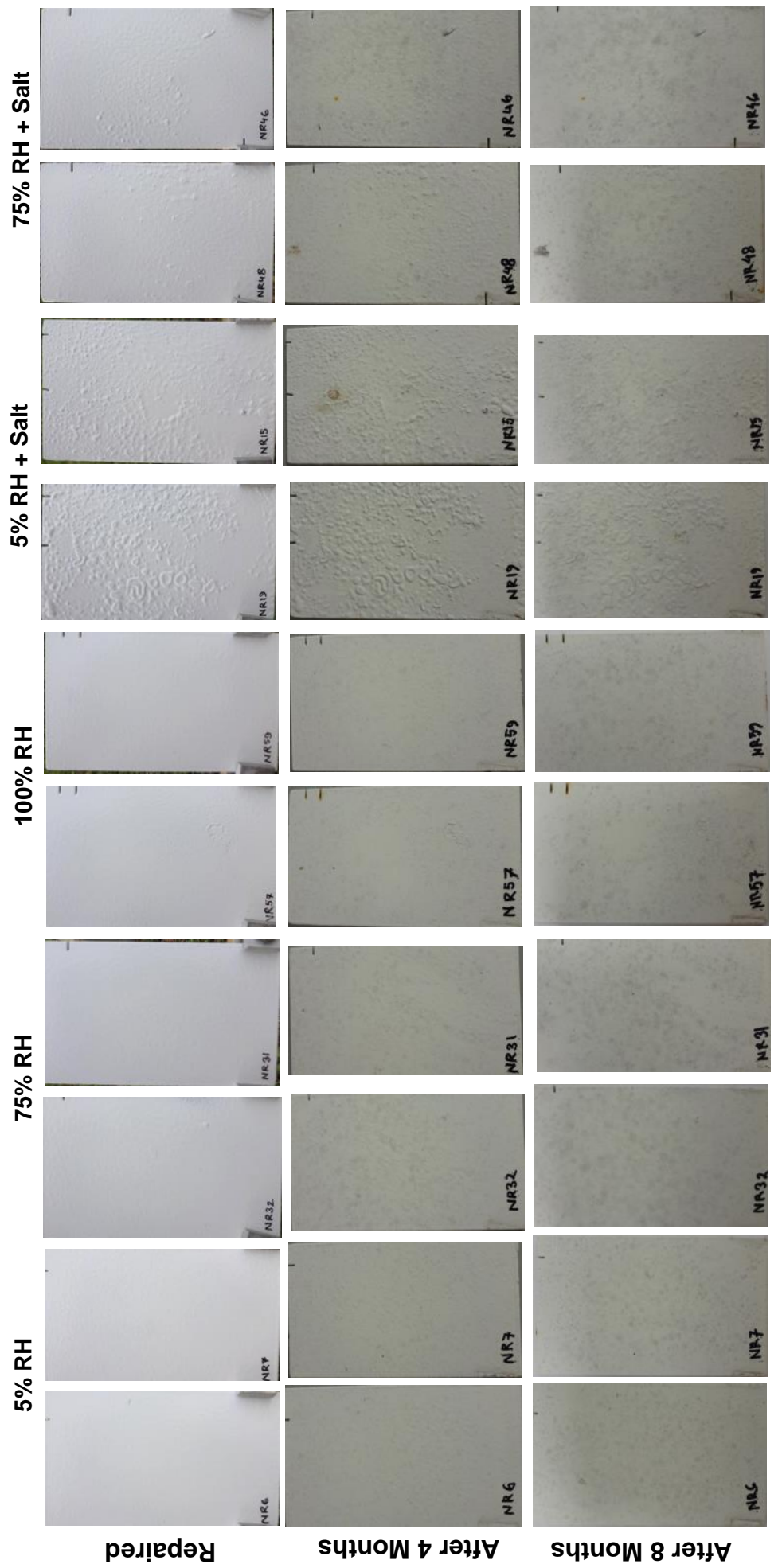
Out-door Exposure NPE-ZRP Repaired Sample (Hand-Cleaned)
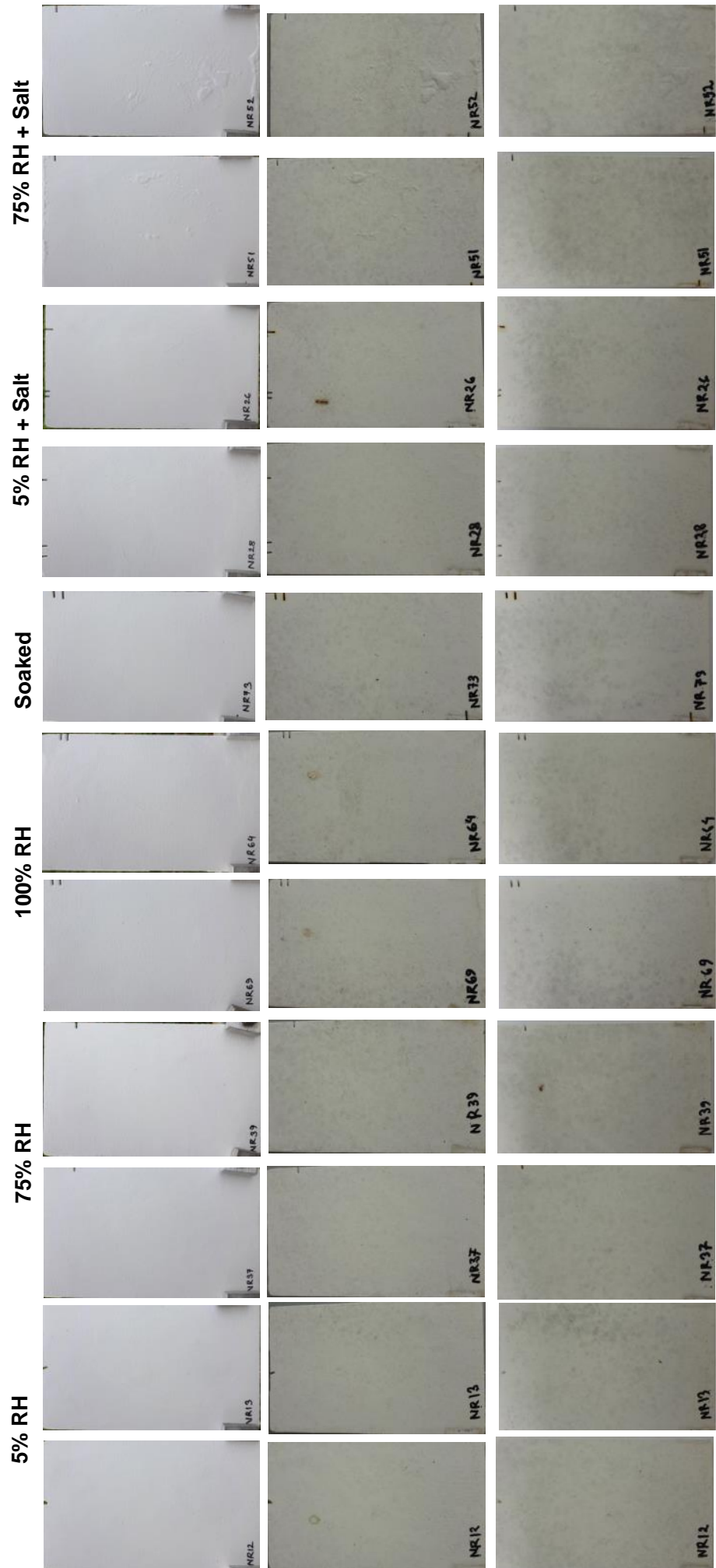

рәл!̣edəy

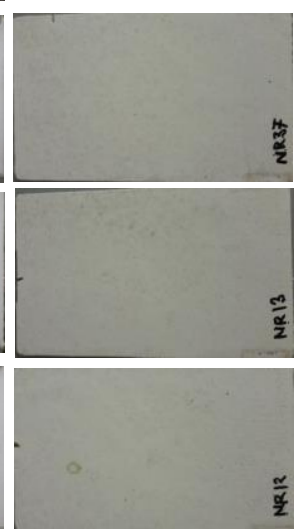

Sप1uoW t גән४
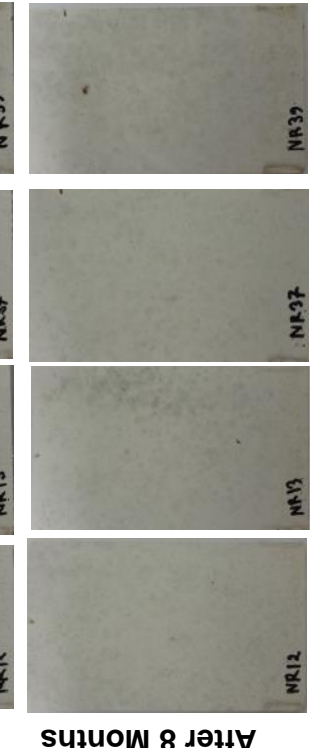
Cyclic Exposure NPE-ZRP Repaired Sample (Sand-blasted + 5\% RH)

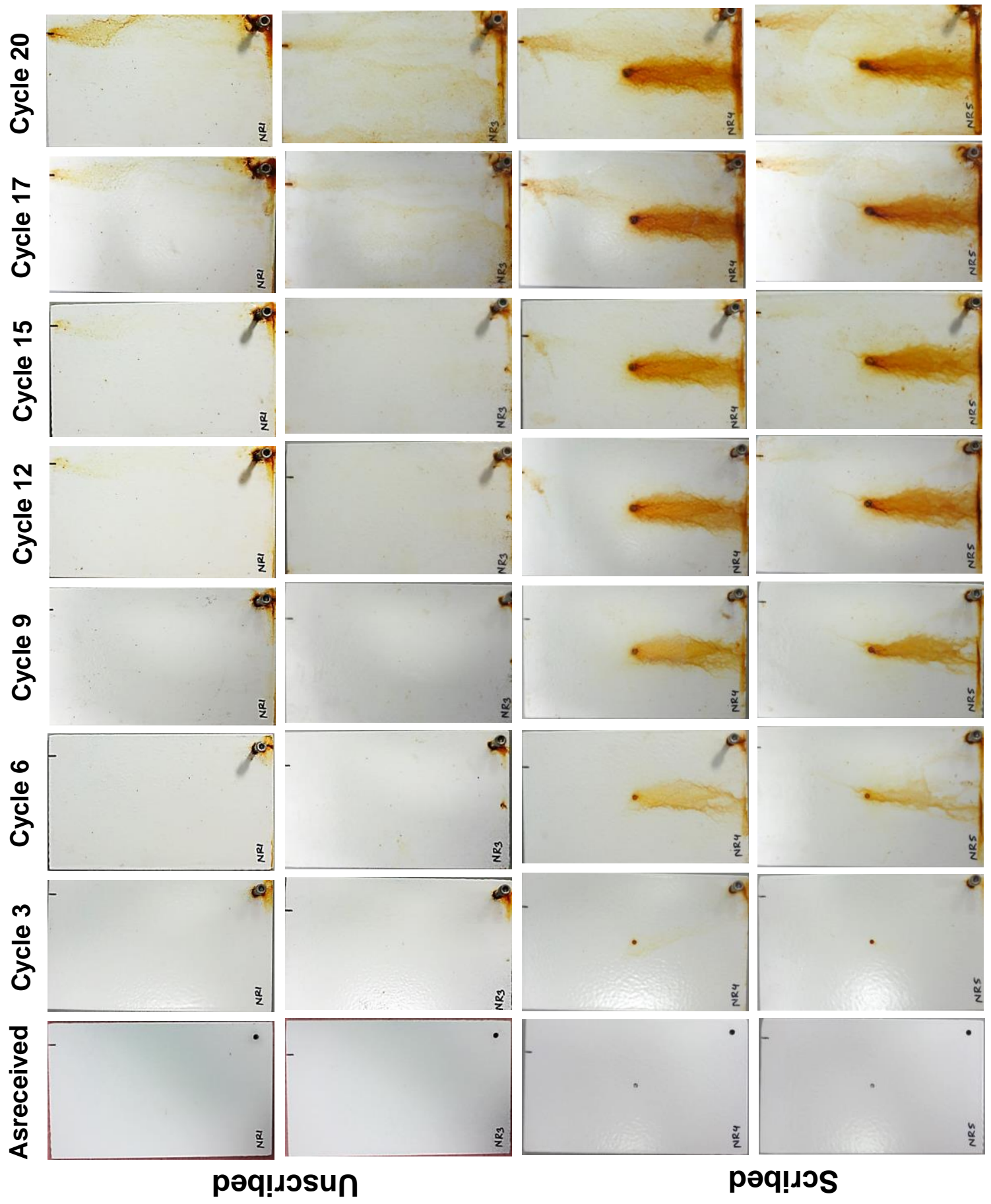


Cyclic Exposure NPE-ZRP Repaired Sample (Sand-blasted + 75\% RH)

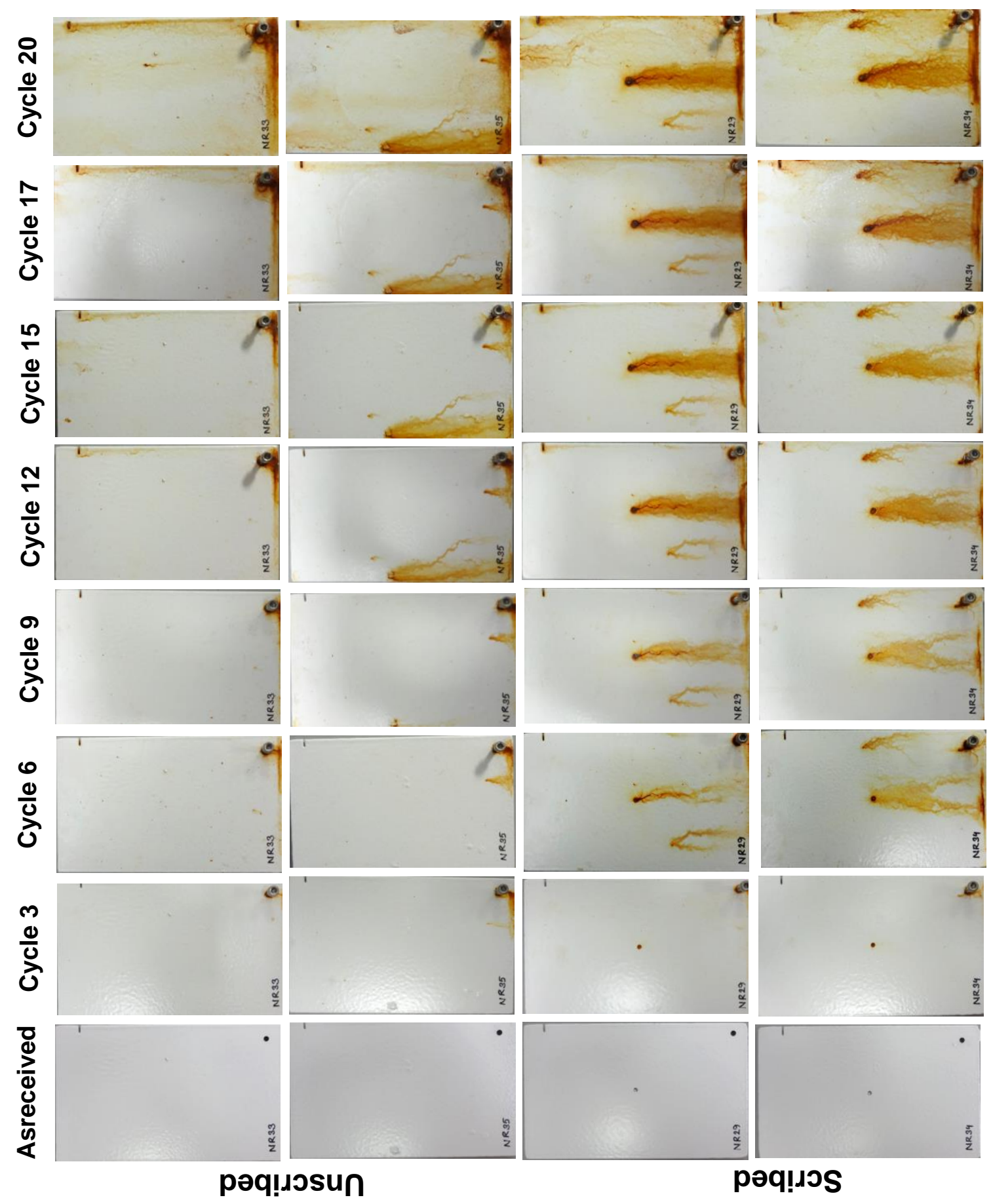


Cyclic Exposure NPE-ZRP Repaired Sample (Sand-blasted + 100\% RH)

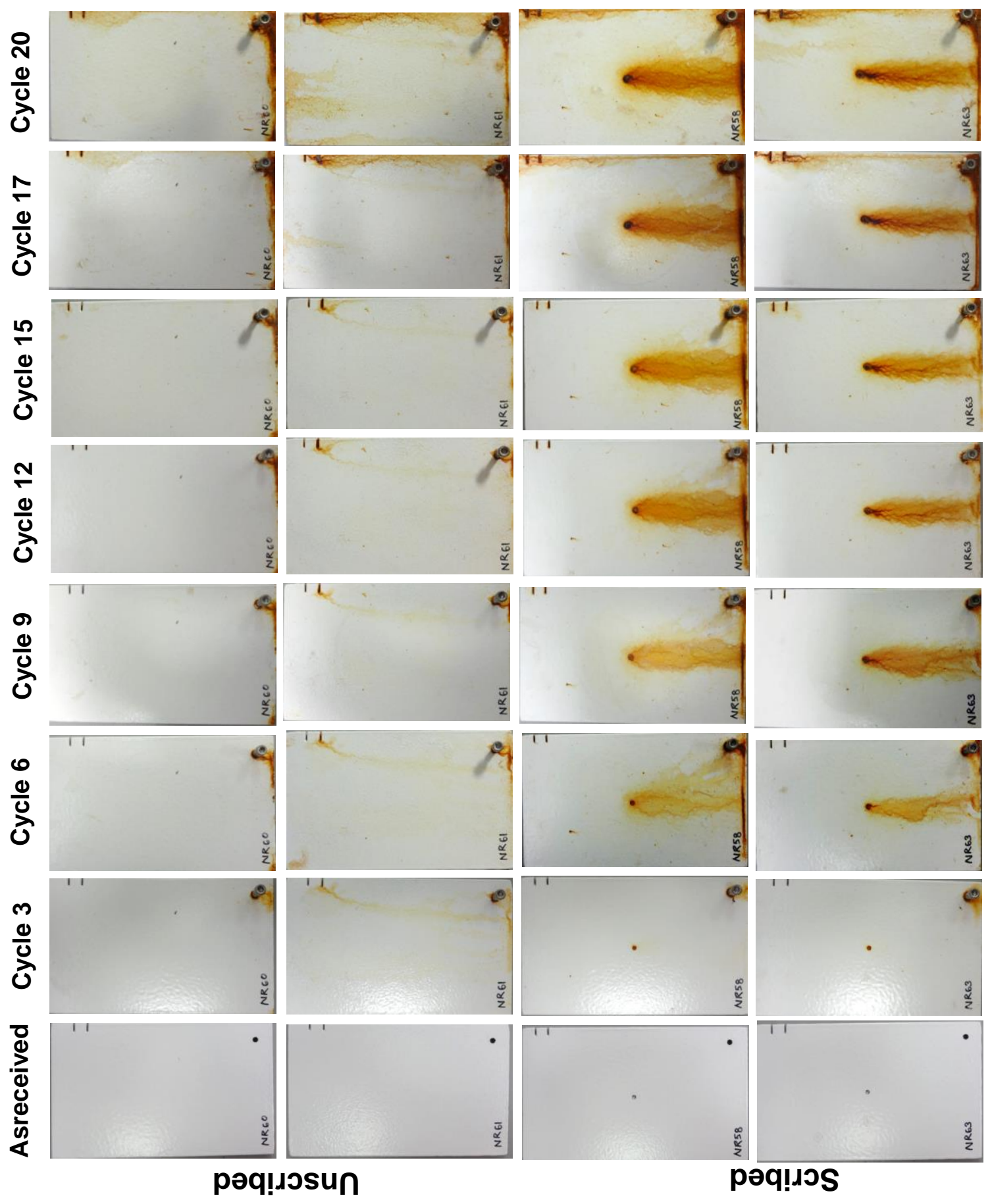


Cyclic Exposure NPE-ZRP Repaired Sample (Sand-blasted + 5\% RH \& Salt)

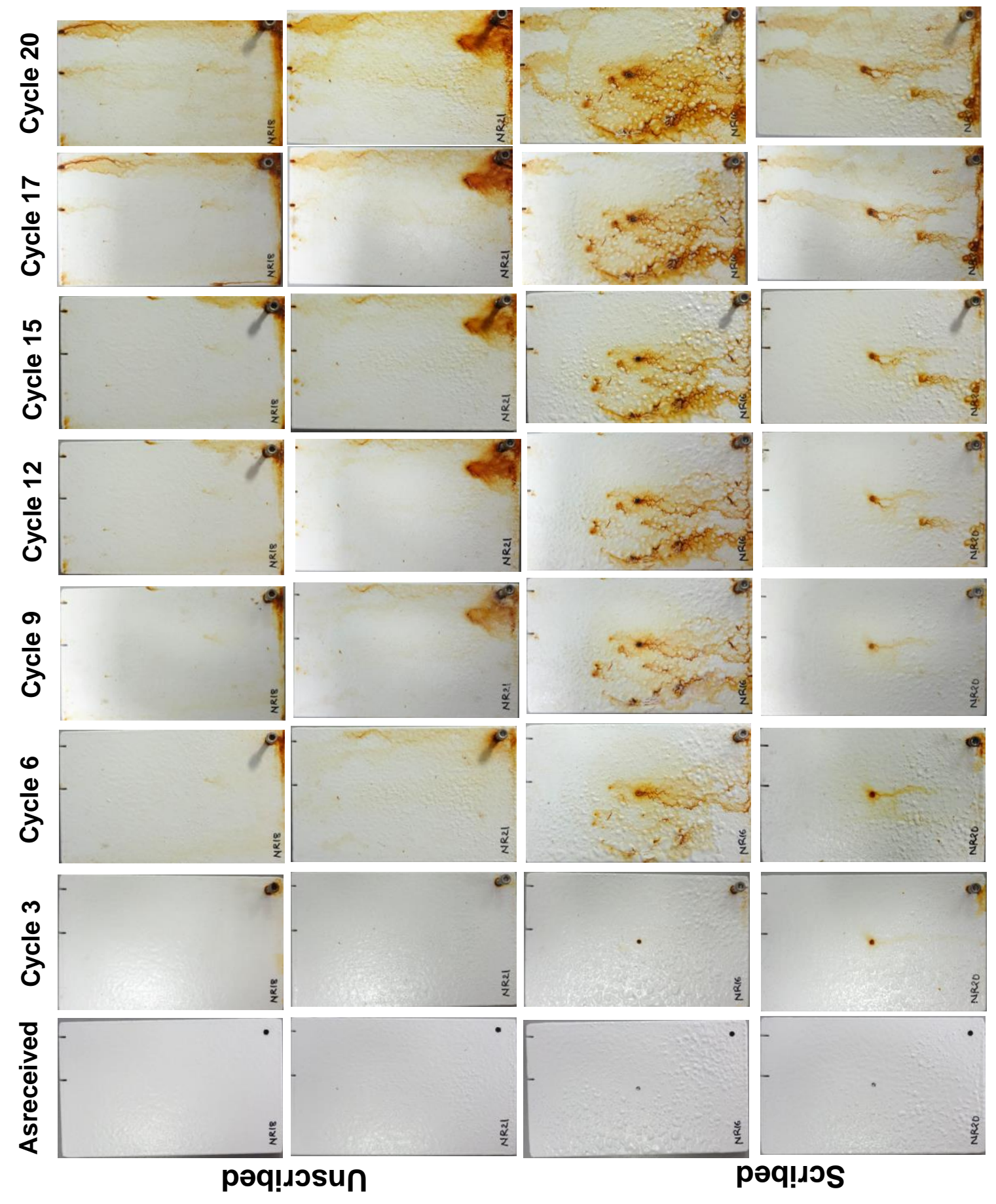


Cyclic Exposure NPE-ZRP Repaired Sample (Sand-blasted + 75\% RH \& Salt)

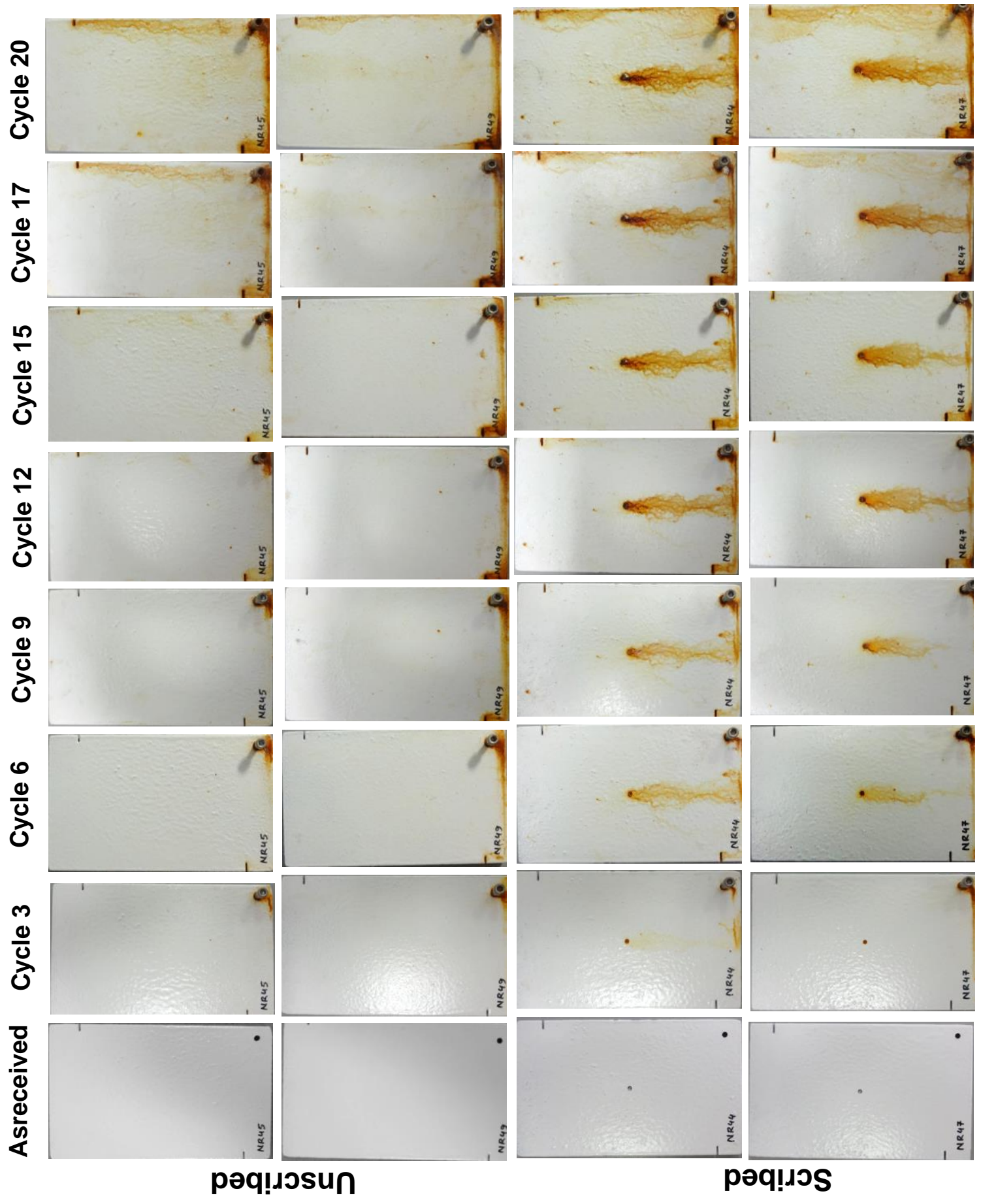


Cyclic Exposure NPE-ZRP Repaired Sample (Hand-cleaned + 5\% RH)

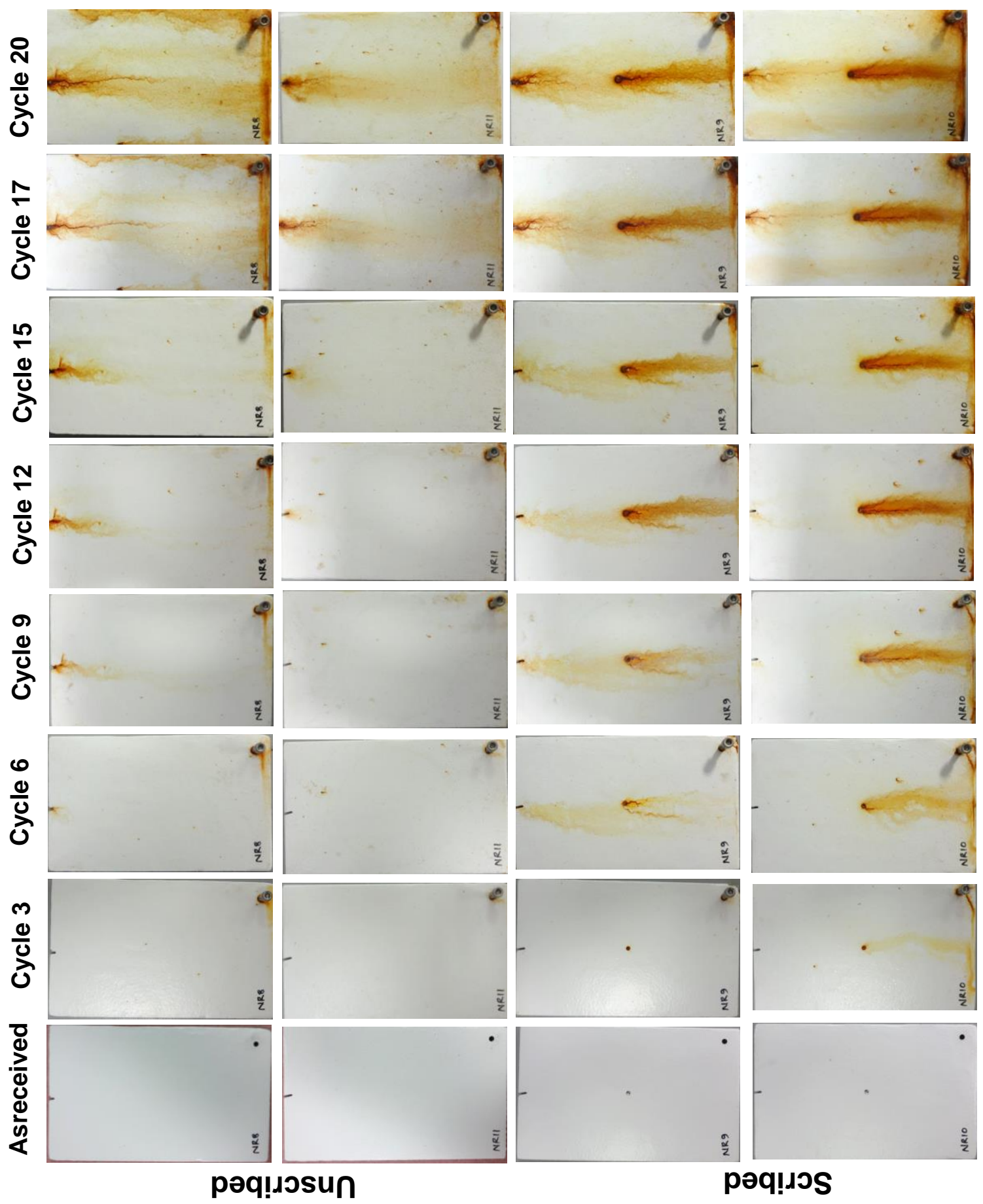


Cyclic Exposure NPE-ZRP Repaired Sample (Hand-cleaned + 75\% RH)

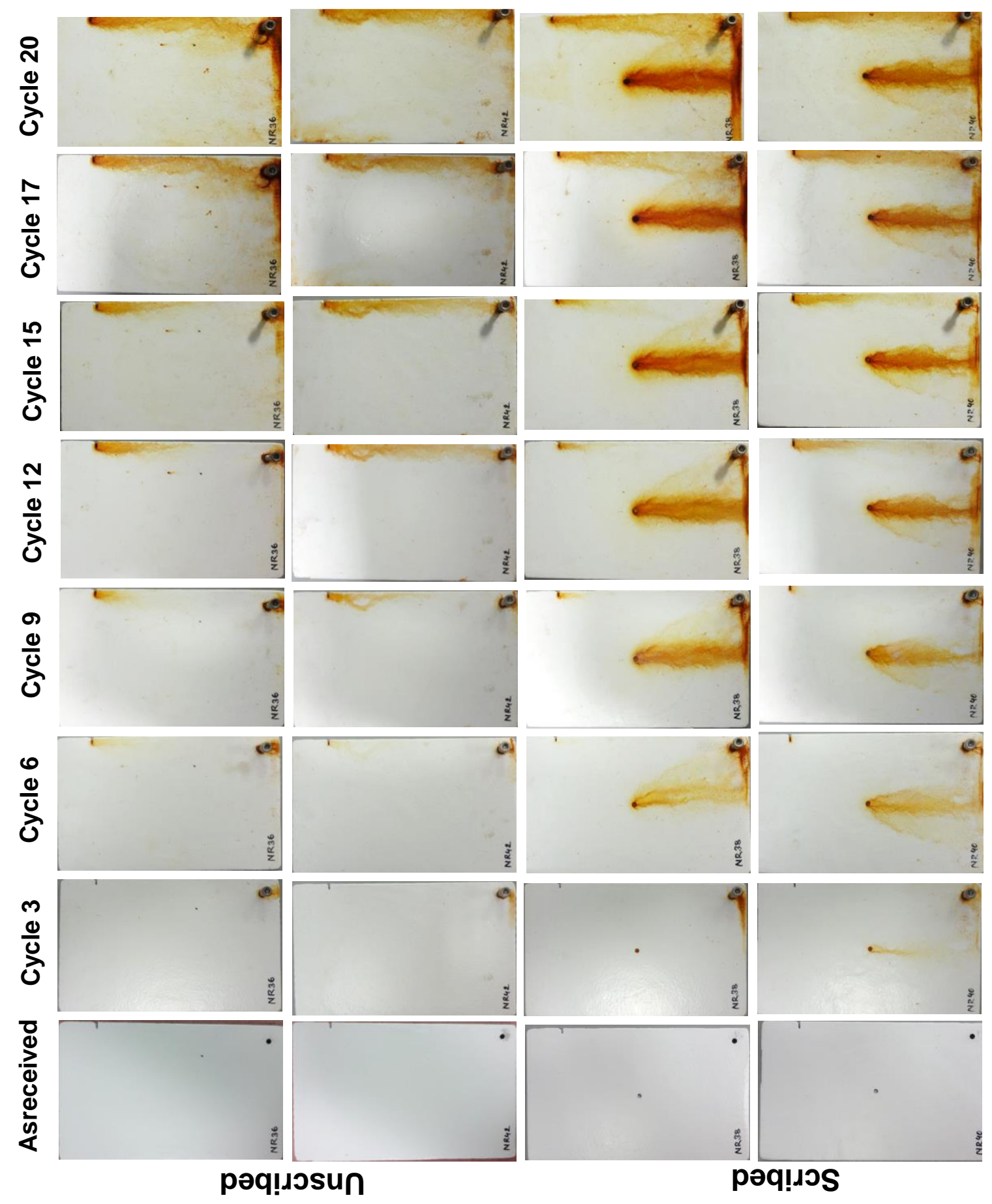


Cyclic Exposure NPE-ZRP Repaired Sample (Hand-cleaned + 100\% RH)

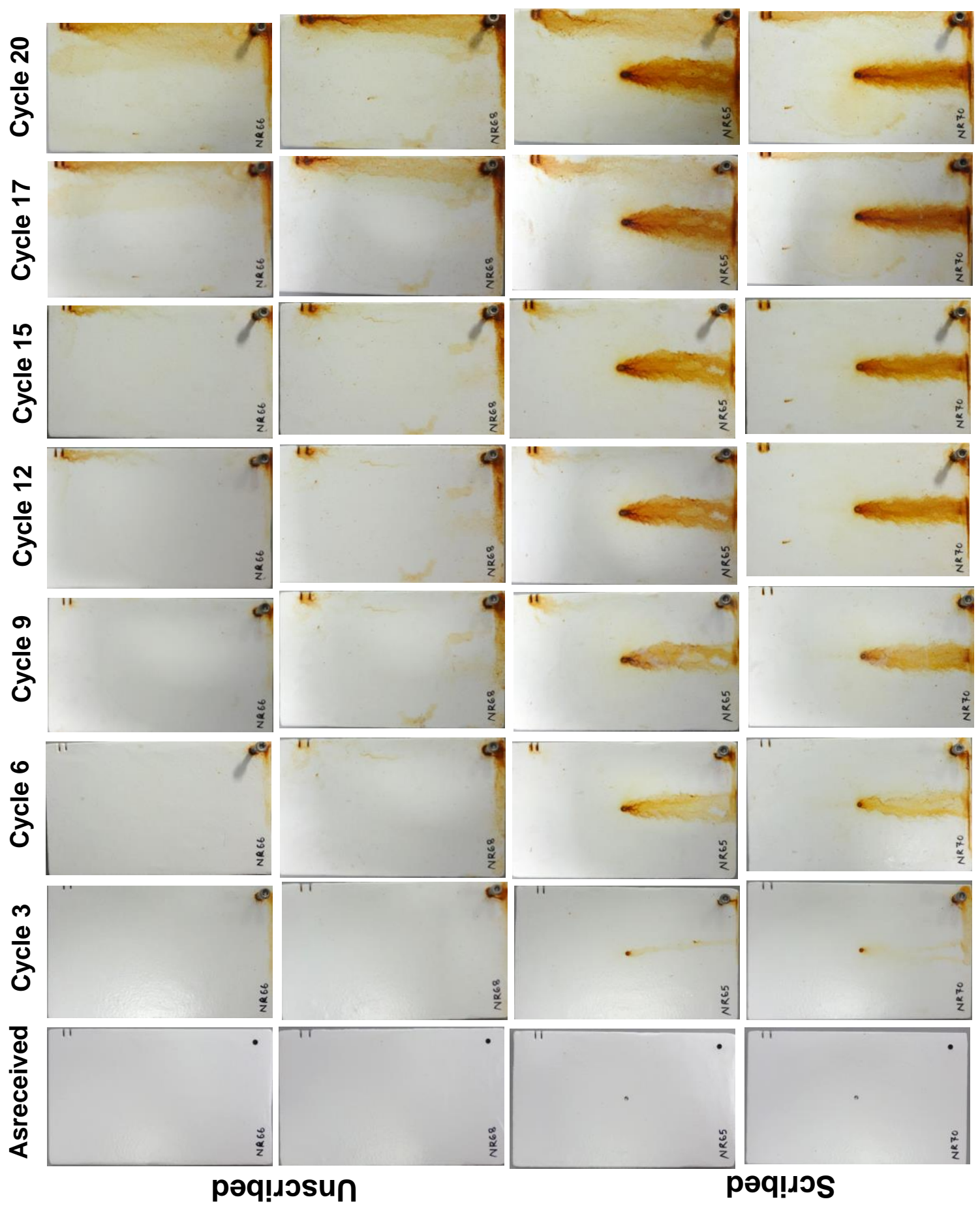


Cyclic Exposure NPE-ZRP Repaired Sample (Hand-cleaned + Soaked)

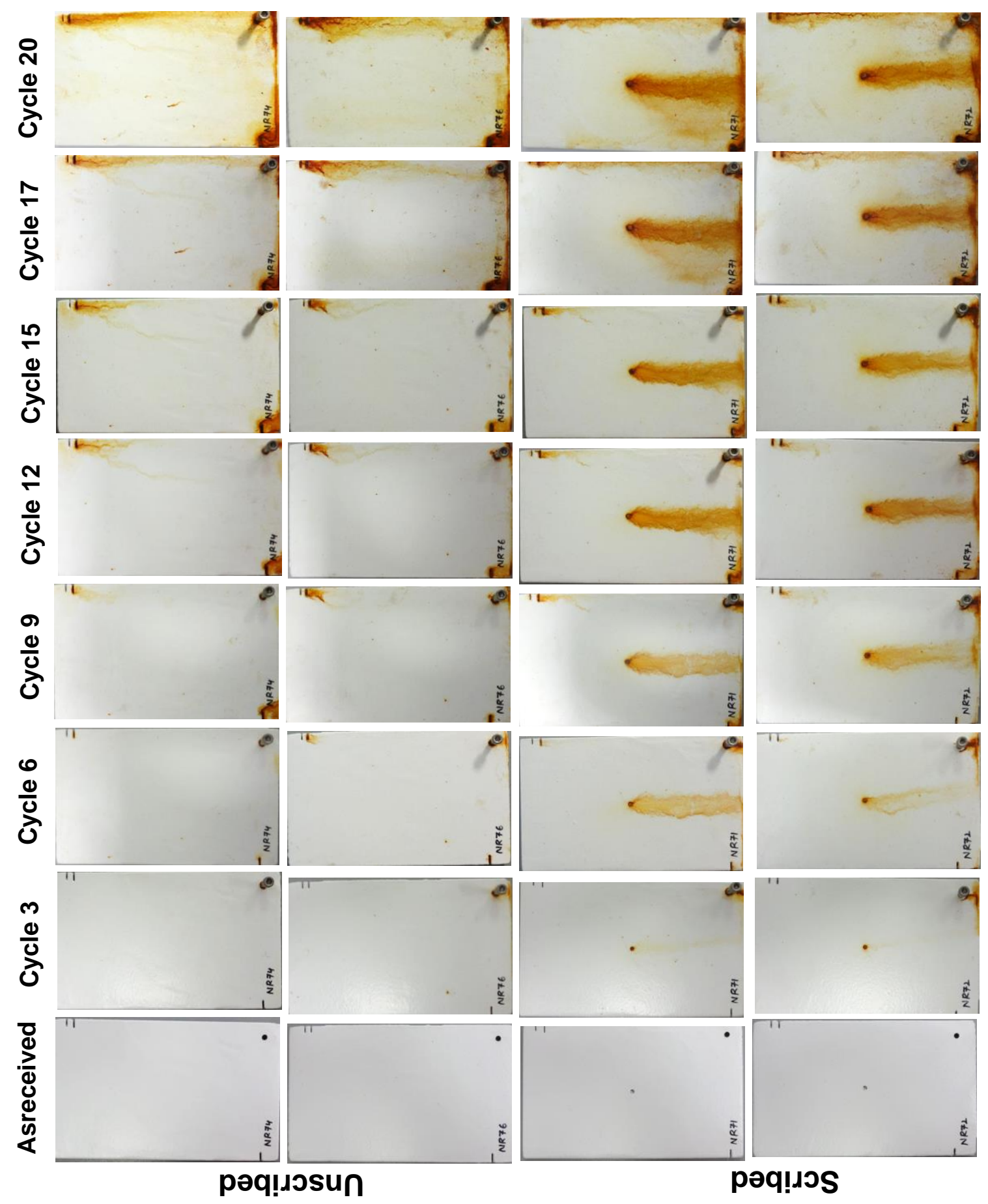


Cyclic Exposure NPE-ZRP Repaired Sample (Hand-cleaned + 5\% RH \& Salt)

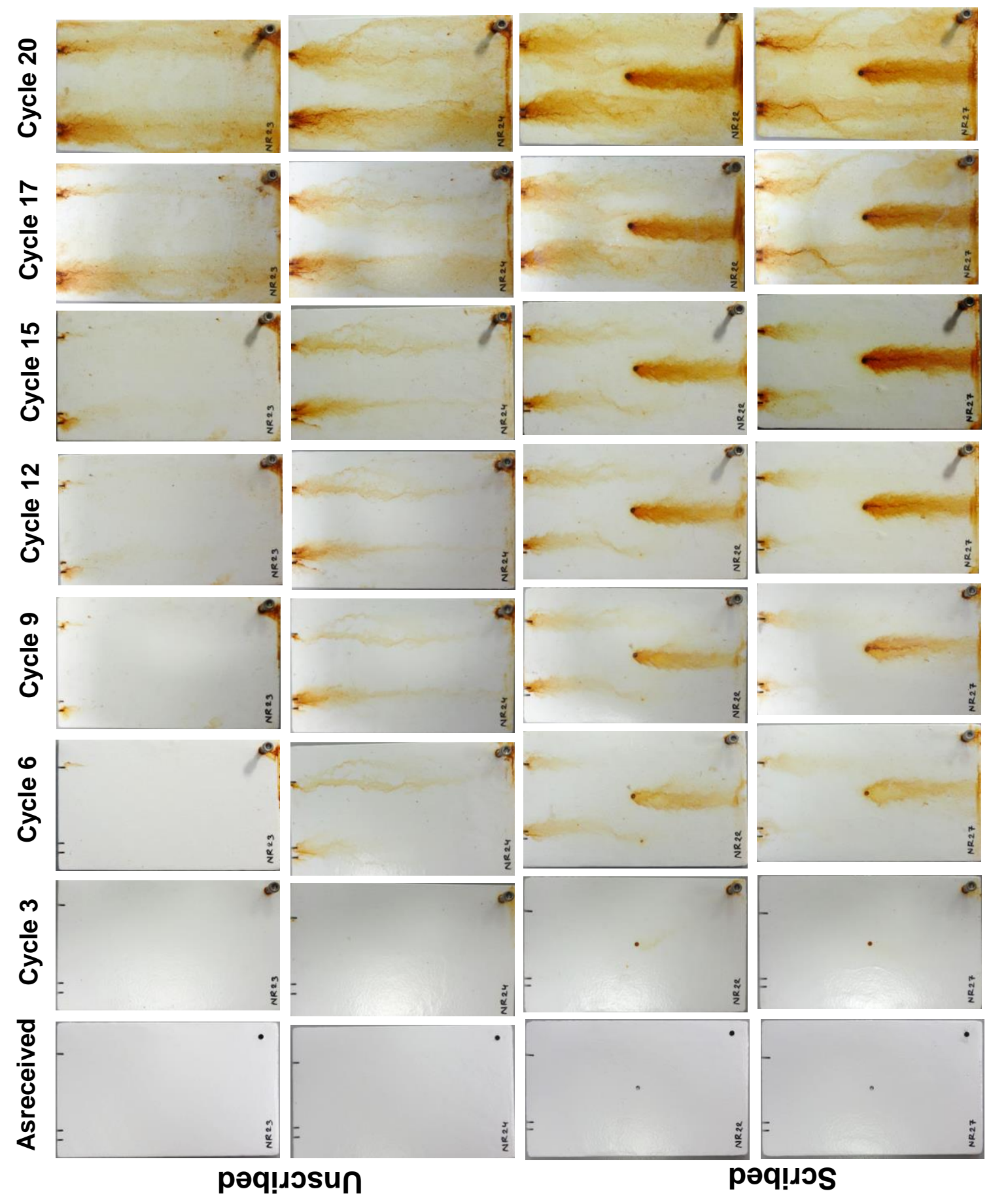


Cyclic Exposure NPE-ZRP Repaired Sample (Hand-cleaned + 75\% RH \& Salt)

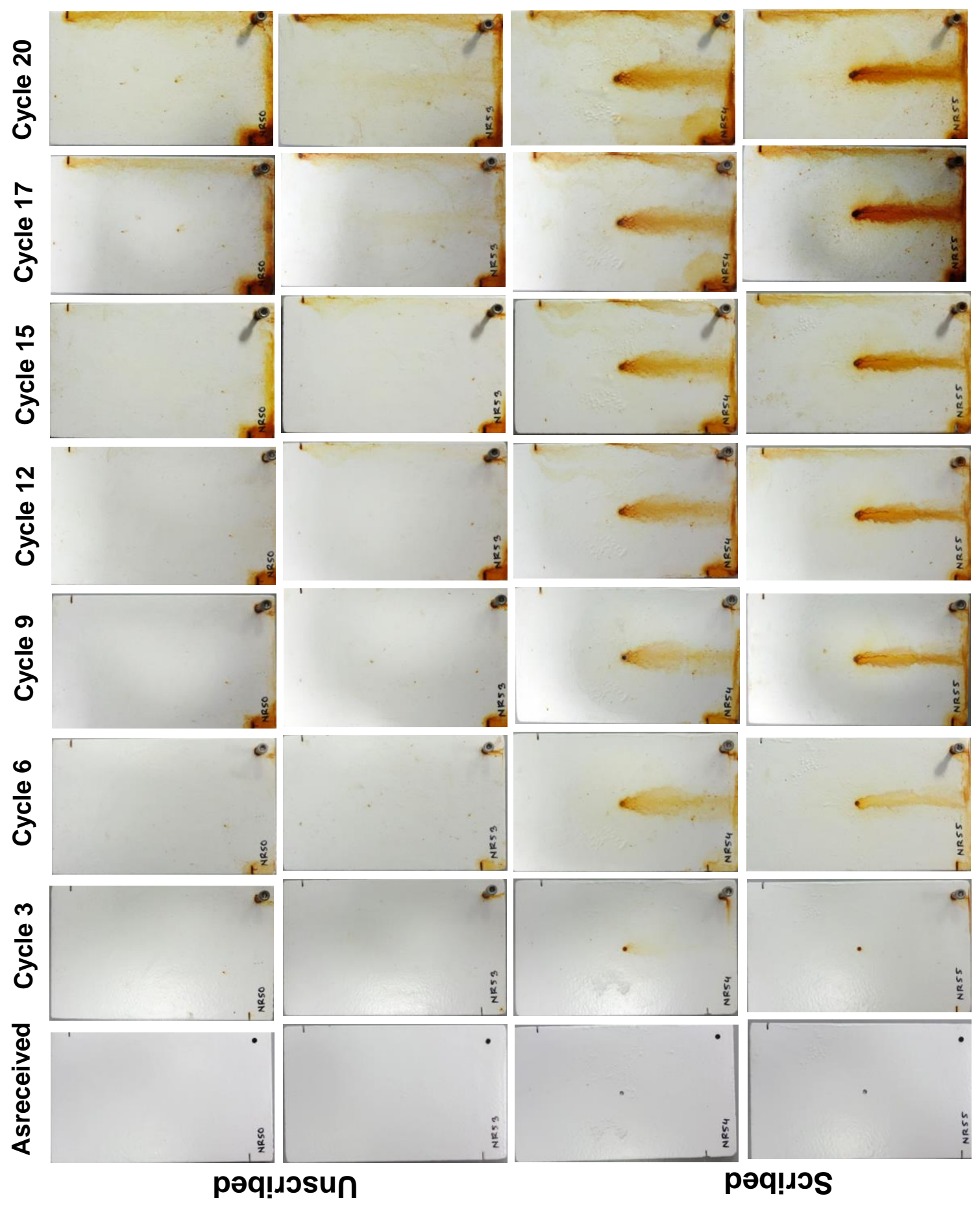


Out-door Exposure ZRP Repaired Sample (Sand-blasted)
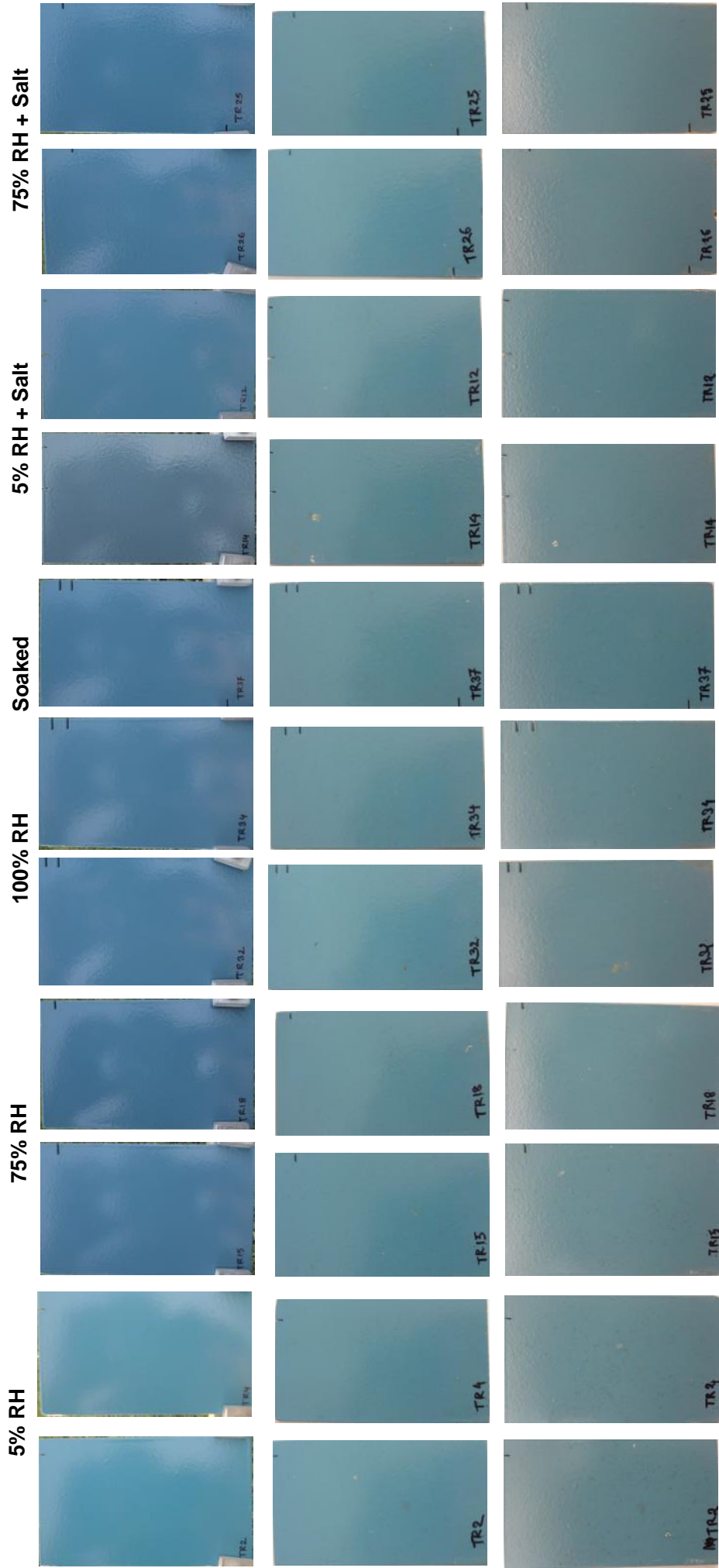

pəג!̣edəy
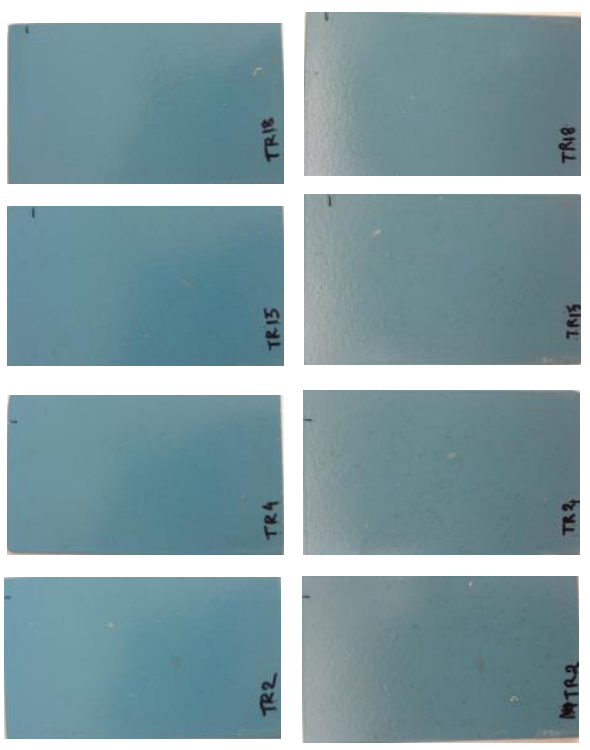

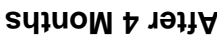

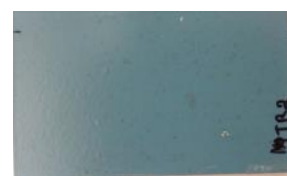

SपIUOW 8 גəH४ 
Cyclic Exposure ZRP Repaired Sample (Sand-blasted + 5\% RH)

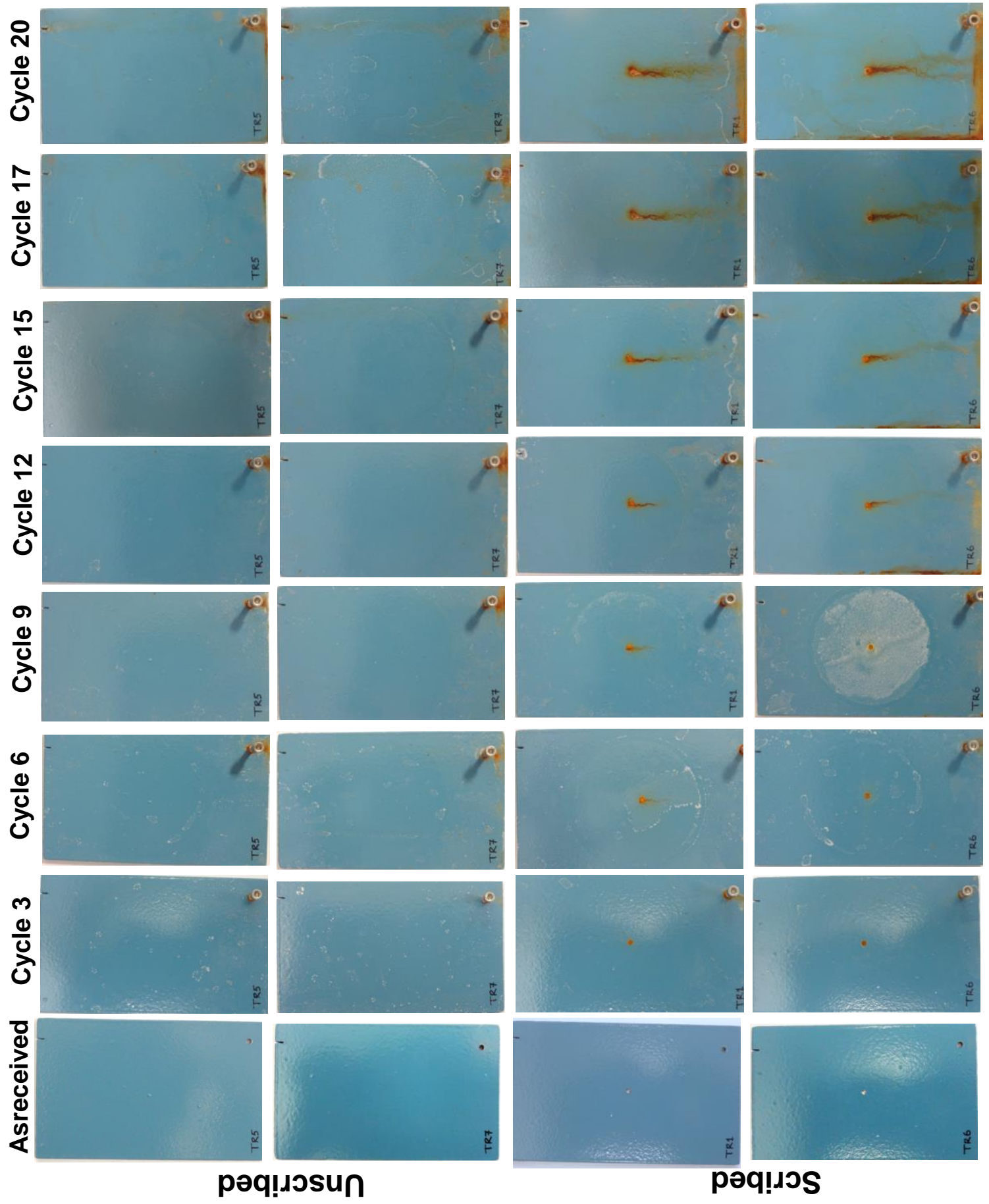


Cyclic Exposure ZRP Repaired Sample (Sand-blasted + 75\% RH)

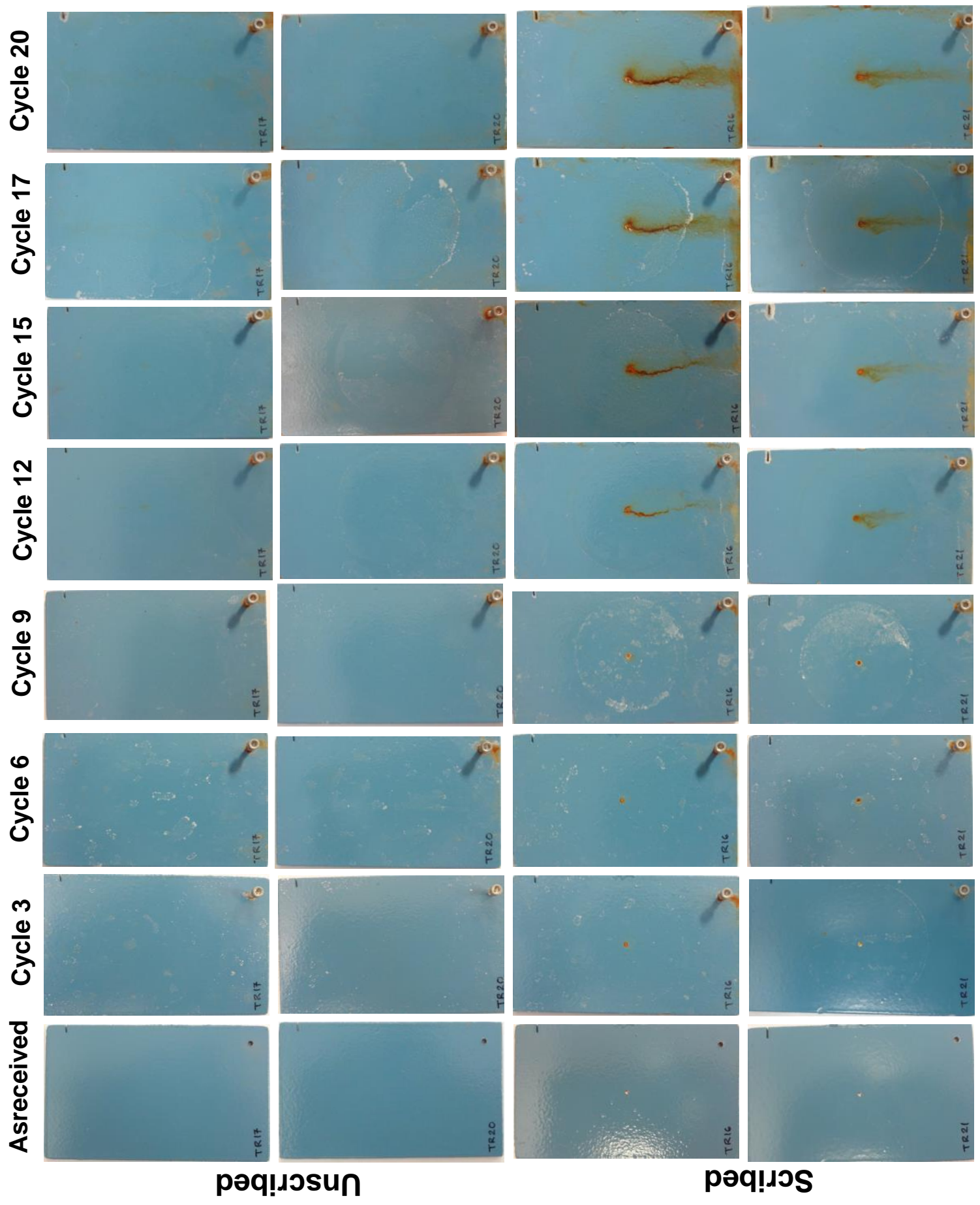


Cyclic Exposure ZRP Repaired Sample (Sand-blasted + 100\% RH)

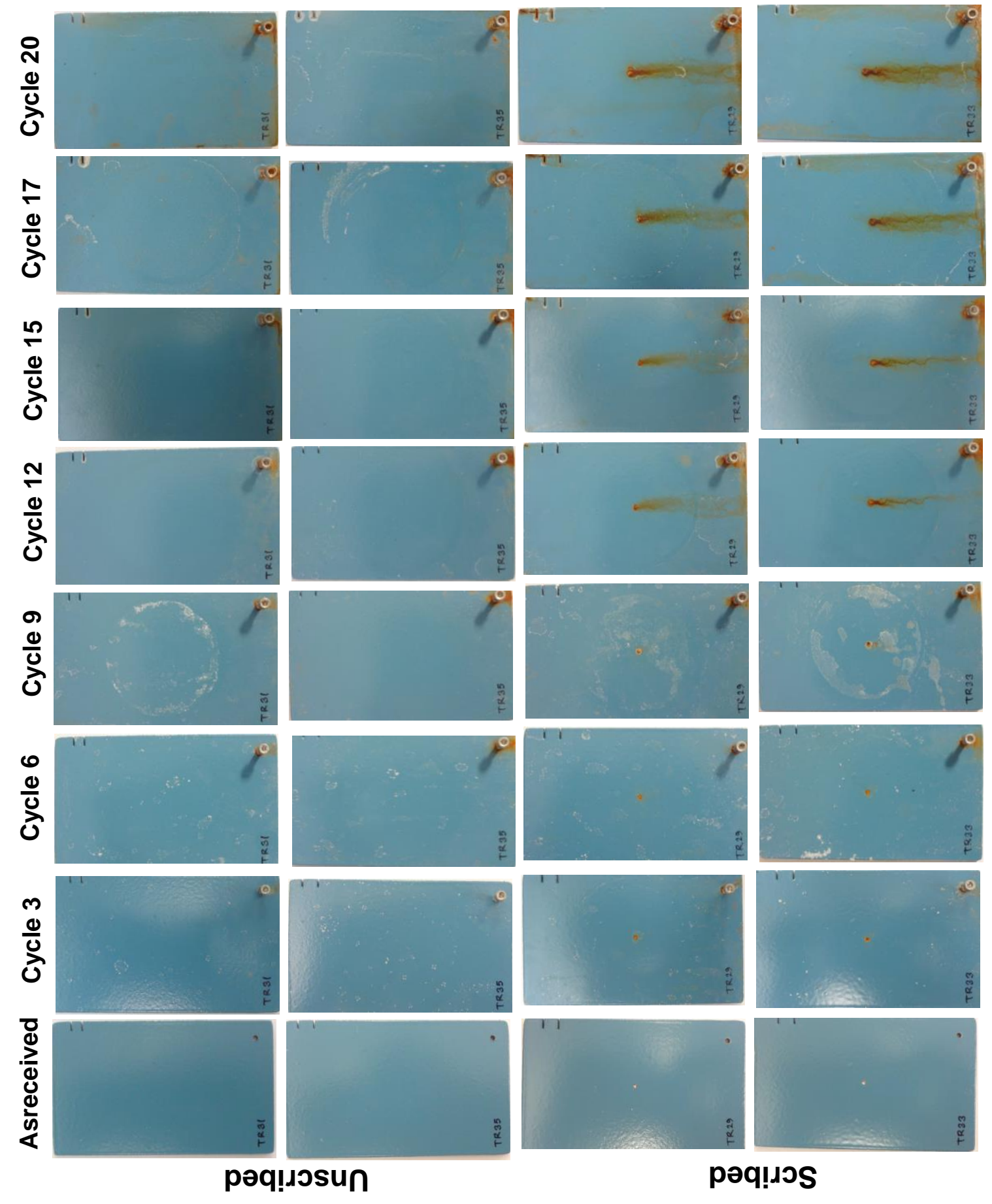


Cyclic Exposure ZRP Repaired Sample (Sand-blasted + Soaked)

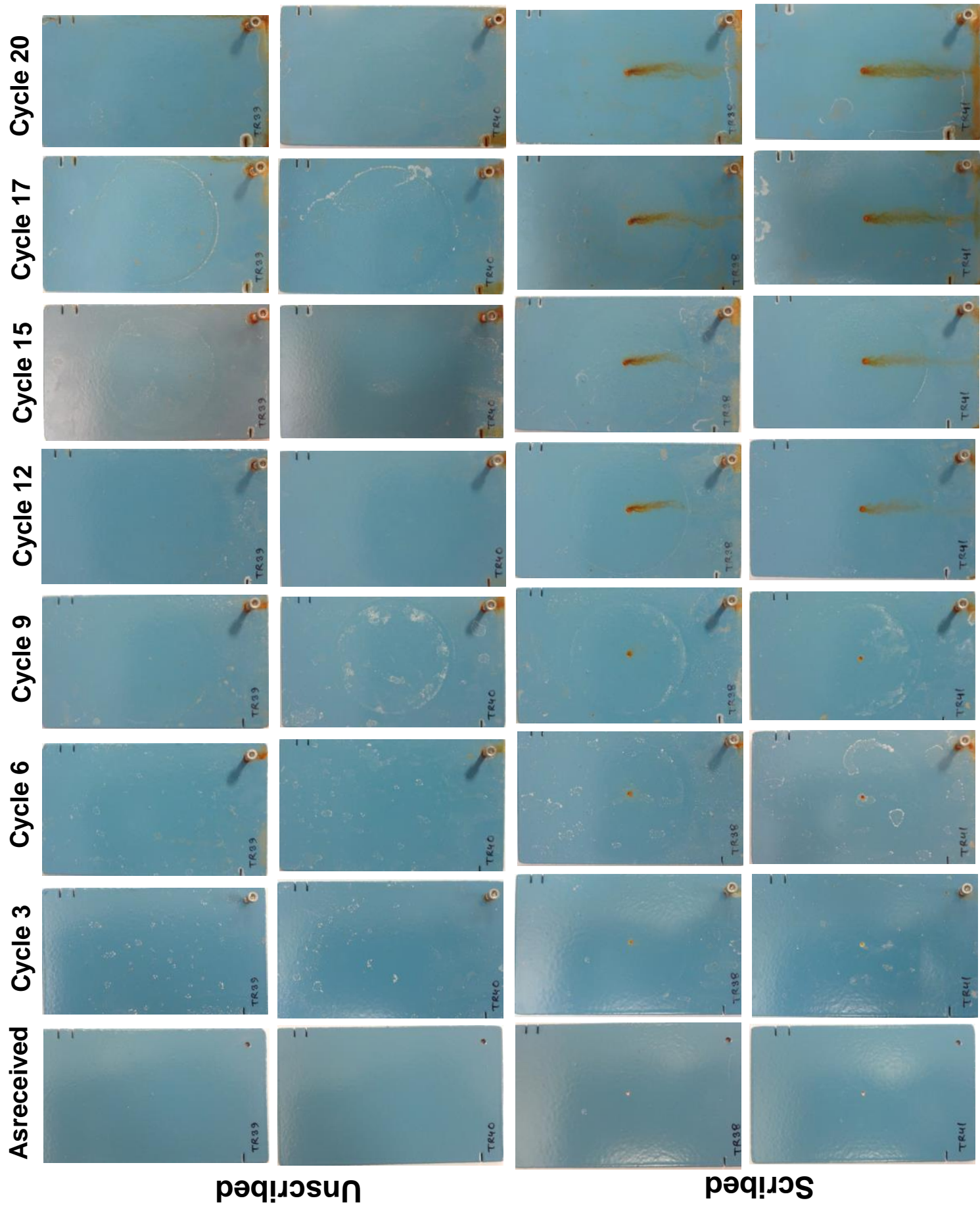


Cyclic Exposure ZRP Repaired Sample (Sand-blasted + 5\% RH \& Salt)

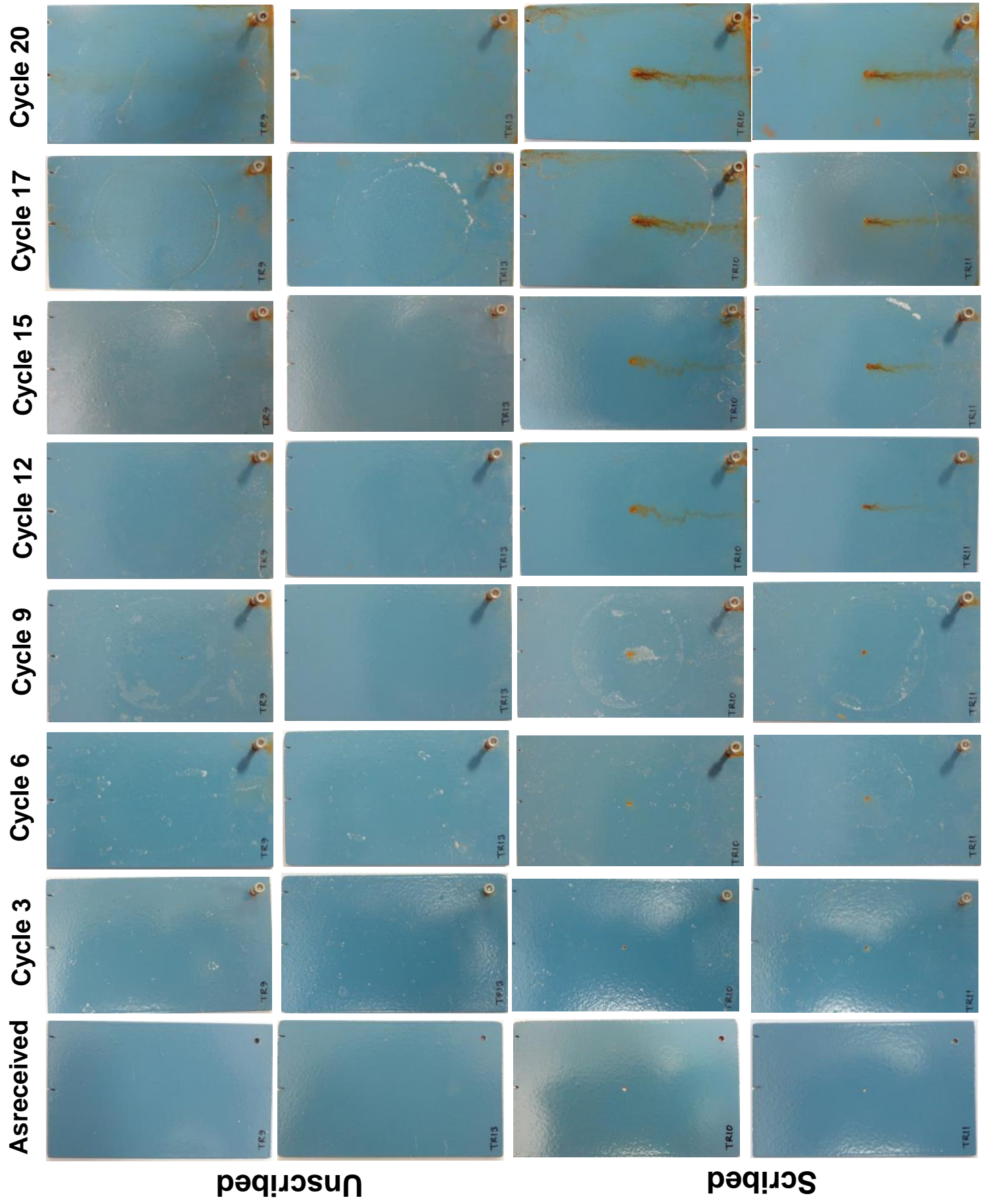


Cyclic Exposure ZRP Repaired Sample (Sand-blasted + 75\% RH \& Salt)

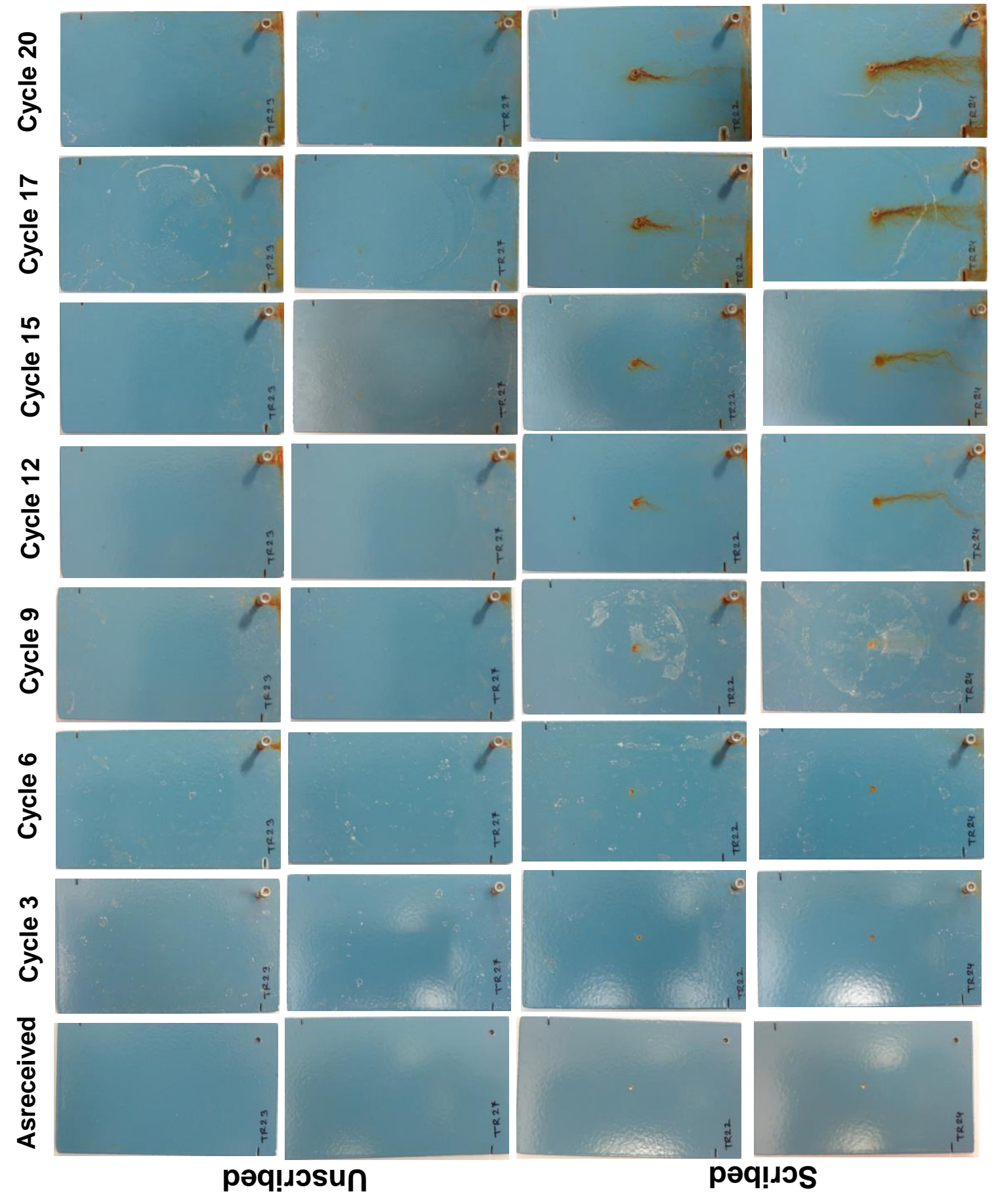




\section{APPENDIX D- Repair Samples Failure Modality}

Pull-off Strength Failure Modality of NPE-ZRP Repaired Samples (Sand-blasted)
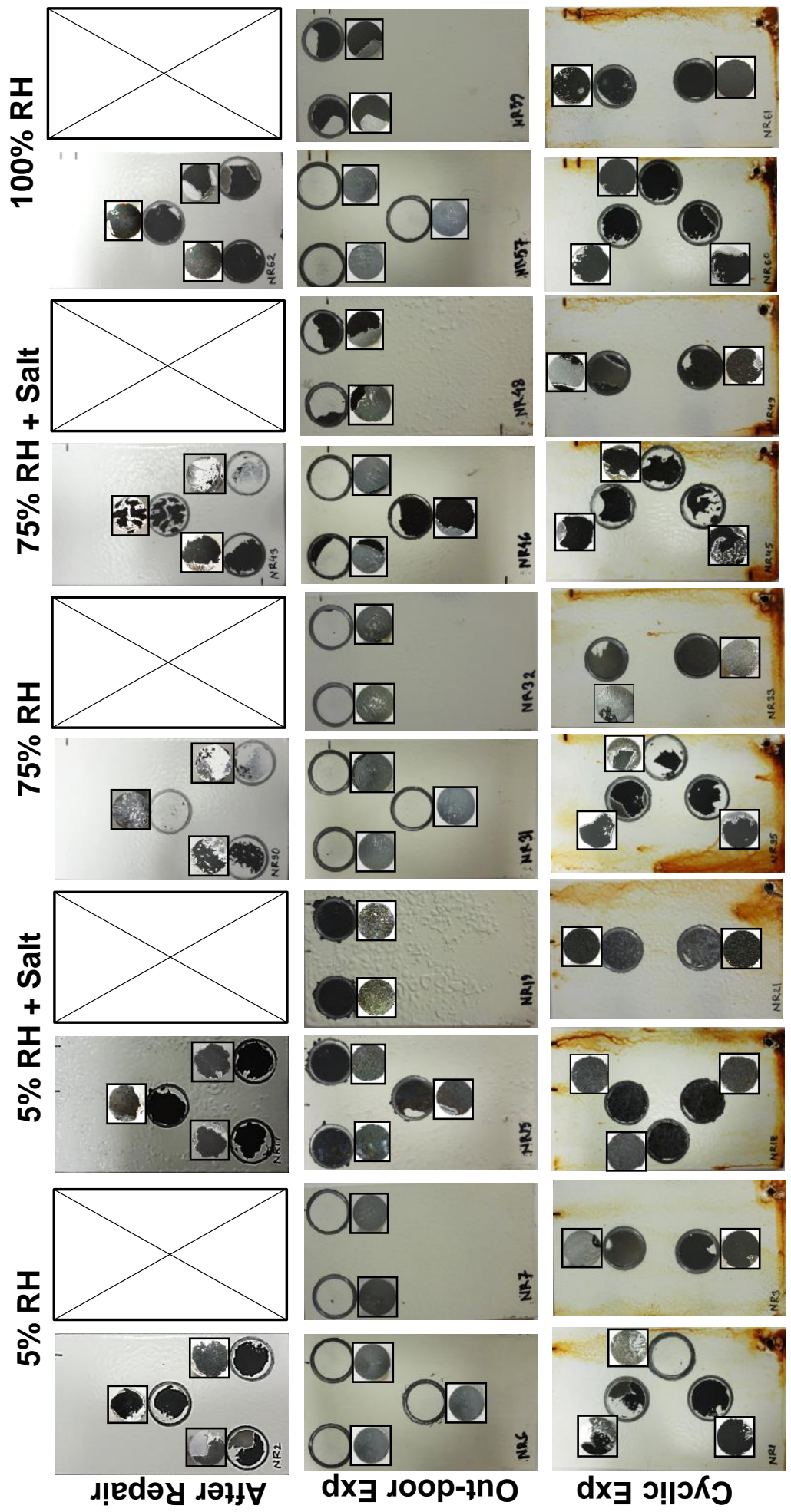
Pull-off Strength Failure Modality of NPE-ZRP Repaired Samples (Hand-cleaned)
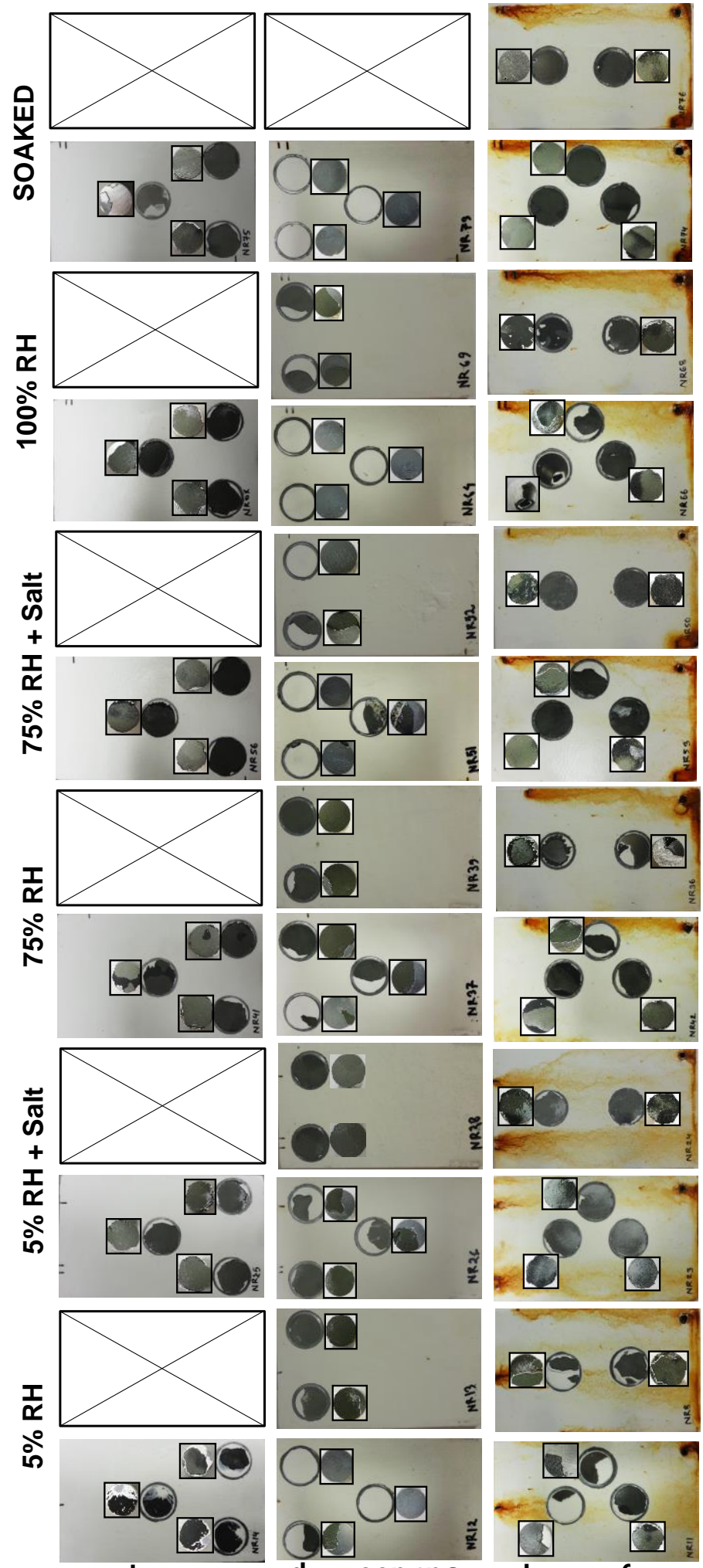

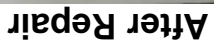
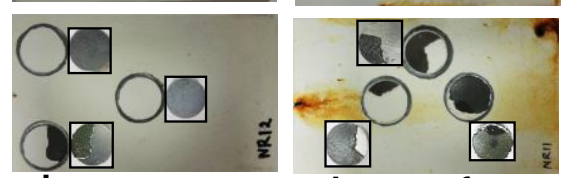

dxヨ toop-ןno

dxヨ ग! 
Pull-off Strength Failure Modality of ZRP Repaired Samples (Sand-blasted)
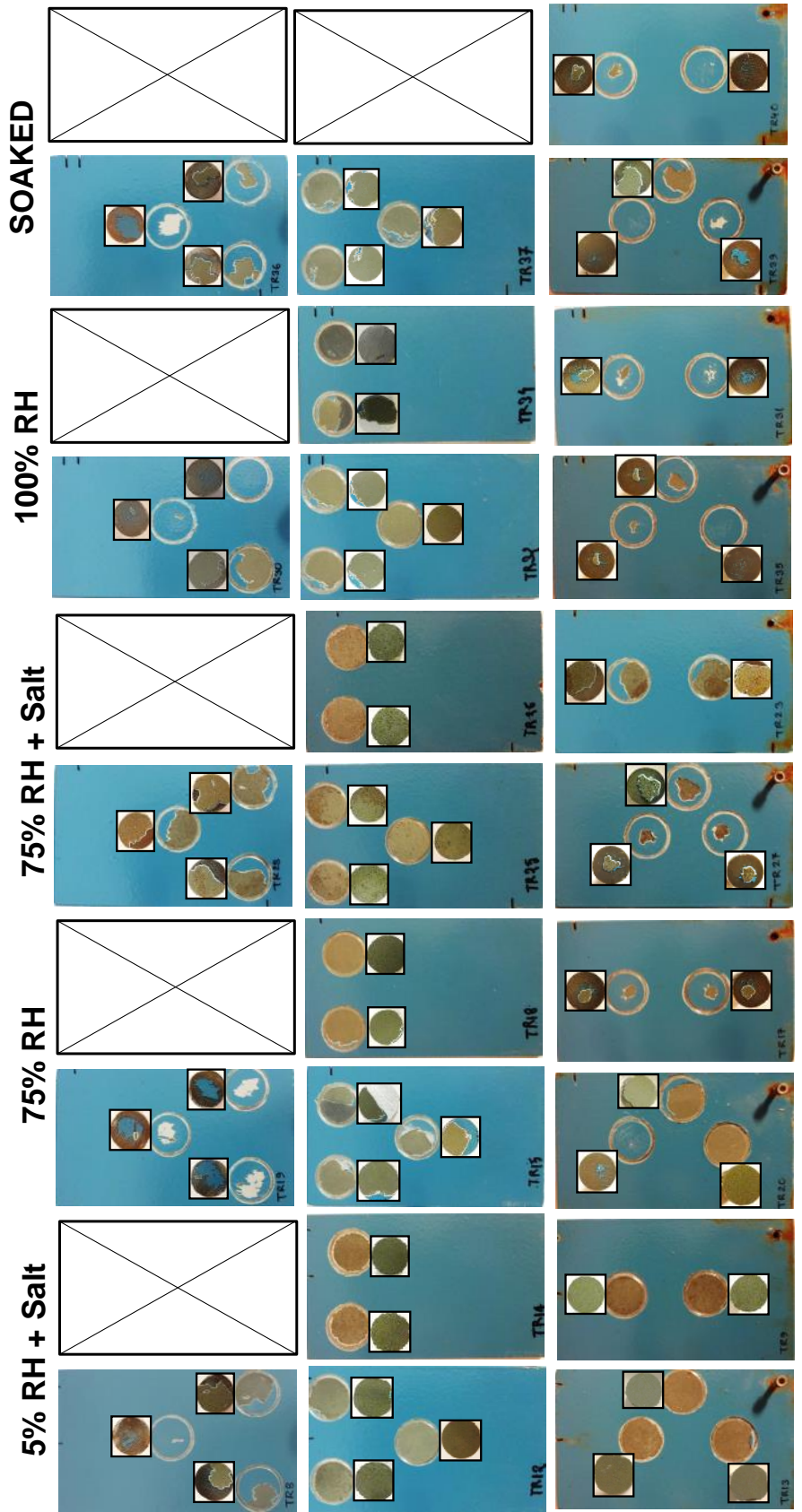

$$
\text { ํํㅇ }
$$
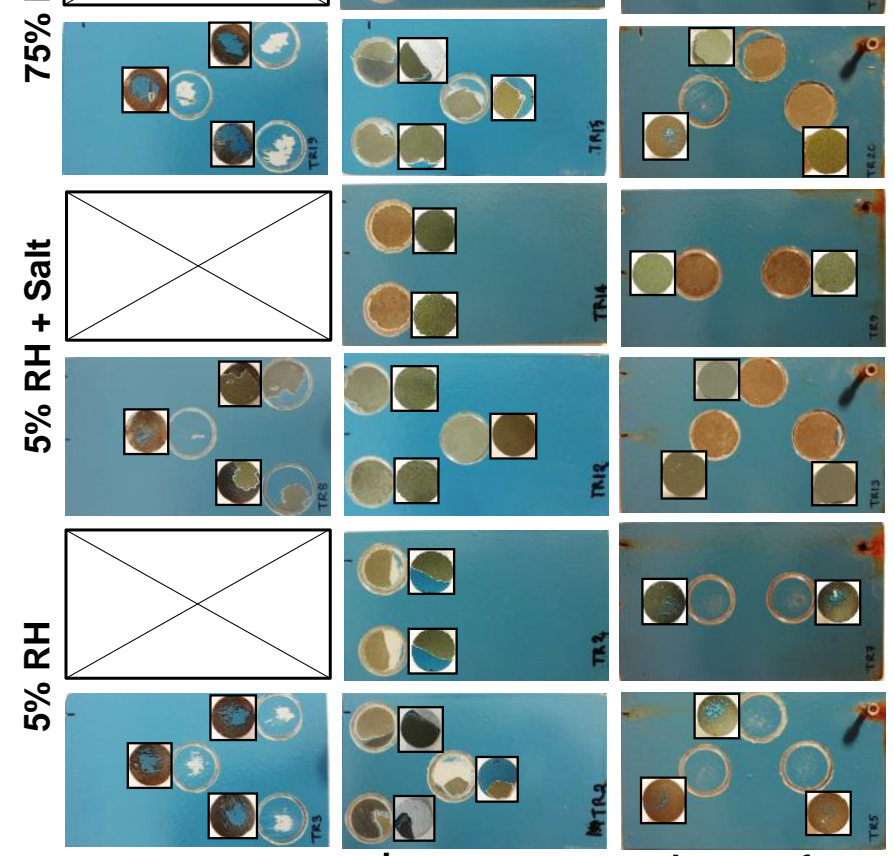

ג!̣edəy ләఘళ
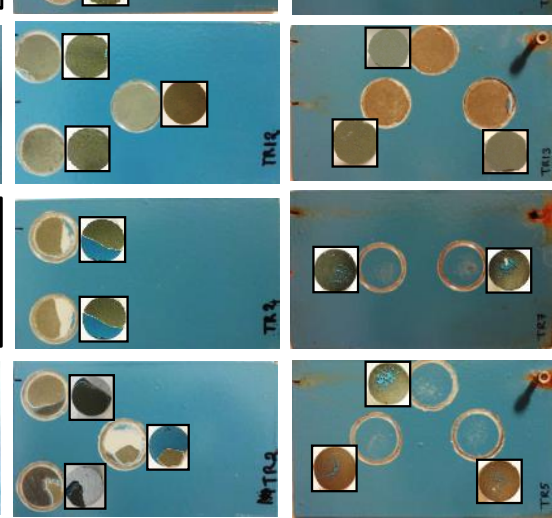

dxヨ toop-ıno

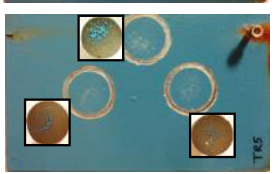

dxヨ ग!๐Кว 
APPENDIX E- Copyright Release 


\section{Permission to Reproduce Figures, Photos, and Tables from Copyrighted Works}

Date: $11 / 13 / 2019$

Name:Saiada Fuadi Fancy

Title: Graduate Research Assistant

Company ("Publisher"): Florida Internationa University

Address: 10555 West Flagler Street, Miami, Fl-33174

Tel: 3053025648

Fax:

Email: sfanc002@fiu.edu

Material to be Reproduced ("Work"): Please provide a complete description of the material involved. Include the following elements where applicable: publication name, issue date, page number, figure/photo/table number, paper number, conference name, etc.

Extended Connectivity of Zinc Pigments to Provide Enhanced Galvanic Coupling by Partial

Replacement with Nanoparticles (51319-13470-SG) \&

Durability of Nano-Coating for Marine Highway Bridge Application ( 51317 --9539-SG)

Reproduction Method ("Publication Media"): Please provide a complete description of how and where the Work will be used. Include the following elements as applicable: publication name, issue, circulation, print run, web site address, conference name, presentation time and place, etc.

PhD Dissertation "Corrosion Duarbility of a Nano-particle Enriched Zinc-rich Coatina Svstem

for Highway Steel Bridges". Florida Internationt University.

NACE International ("NACE") hereby grants to "Publisher" the right to publish the Work utilizing the Publication Media described above. The publication right granted herein is limited the specific Publication Media described above. To the extent the publication of the Work is to occur by an above-specified date, such as in a particular periodical or at a particular conference, the right granted herein shall automatically terminate upon the date of such occurrence, whether or not the Work is actually published. To the extent the Publication Media is a web site, the publication right is limited to publication at the specific web site URL identified above. Any right granted herein is a limited, non-transferable, non-exclusive right. No other rights in the Work are granted herein.

Publisher shall not edit or modify the Work except to meet the style and graphic requirements of the individual media involved.

The Publisher shall include the following notation with any publication of the Work:*

\section{A. Conference Paper}

Reproduced with permission from NACE International, Houston, TX. All rights reserved. Author(s), Paper NUMBER presented at CORROSIONYYEAR, City, State. @ NACE International FIRST YEAR OF PUBLICATION.

\section{B. Journal Article}

Reproduced with permission from NACE International, Houston, TX. All rights reserved. Author(s) name, Article title, Journal title, Vol. no., Issue no., and publication year, (5) NACE International FIRST YEAR OF PUBLICATION.

\section{Magazine Article}

Reproduced with permission from NACE International, Houston, TX. All rights reserved. Author(s) name, Article title, Magazine title, Vol. no., Issue no., and publication year. (C) NACE International FIRST YEAR OF PUBLICATION

\section{Standards}

STANDARDS/TECHNICAL COMMITTEE REPORT NAME. (O) NACE International YEAR. All rights reserved by NACE. Reprinted with permission. NACE standards are revised periodically. Users are cautioned to obtain the latest edition; information in an outdated version of the standard may not be accurate.

* Modifications to Notations: Other reference wording can be used, but must be approved by NACE in writing in advance. 
The Publisher shall include full bibliographic citations of or references to the original NACE source, where applicable.

Publisher shall obtain a copy of the original Work directly from NACE International and shall not utilize copies of the Work from other sources, including the author(s).

To the extent the Work is published on a web site as authorized herein, the Work shall be posted in a file format that does not allow the content of the Work to be easily copied from the Web Site or changed.

As between NACE and Publisher, Publisher acknowledges that NACE owns all rights in the Works.

Publisher shall not be entitled to any compensation for its efforts in promoting the Work.

THE WORK IS PROVIDED “AS IS." ALL EXPRESS OR IMPLIED COVENANTS, CONDITIONS, REPRESENTATIONS OR WARRANTIES, INCLUDING ANY IMPLIED WARRANTY OF MERCHANTABILITY OR FITNESS FOR A PARTICULAR PURPOSE OR CONDITIONS OF ACCURACY, COMPLETENESS OR QUALITY AND THOSE ARISING BY STATUTE OR OTHERWISE IN LAW, ARE HEREBY DISCLAIMED.

IN NO EVENT WILL NACE BE LIABLE FOR ANY DIRECT, INDIRECT, PUNITIVE, SPECIAL, INCIDENTAL OR CONSEQUENTIAL DAMAGES IN CONNECTION WITH OR RELATED TO THIS AGREEMENT (INCLUDING LOSS OF PROFITS, USE, DATA, OR OTHER ECONOMIC ADVANTAGE), HOWSOEVER ARISING.

This Agreement and the rights granted herein may be terminated immediately by NACE upon breach of this Agreement by Publisher. Unless earlier terminated, this Agreement and the rights granted herein will automatically terminate 6 months from the Date set forth above. If the Work has not been published within that time period, a new Agreement must be obtained.

Publisher may not, directly or indirectly, sell, assign, sublicense, lease, rent, distribute, or otherwise transfer this Agreement or any rights granted herein, without the prior written consent of NACE.

If any provision of this Agreement is found to be unenforceable, then this Agreement shall be deemed to be amended by modifying such provision to the extent necessary to make it legal and enforceable while preserving its intent. The remainder of this Agreement shall not be affected by such modification.

This Agreement does not create, and shall not be construed to create, any employer-employee, joint venture or partnership relationship between the parties. No officer, employee, agent, servant or independent contractor of either party shall at any time be deemed to be an employee, servant, agent or contractor of any other party for any purpose whatsoever.

This Agreement shall be governed by, and construed and enforced in accordance with, the laws of the State of Texas, without regard to the choice of law provisions of that State.

This Agreement shall only be effective if signed by authorized representatives of both parties. This Agreement constitutes the entire Agreement between the parties with respect to the subject matter of this Agreement. Any change, modification or waiver hereto must be in writing and signed by authorized representatives of both parties.

Other Terms \& Conditions:

Publisher hereby requests permission to publish the Work described above and agrees to comply with all Terms and Conditions listed above.

Request submitted by:

Saiada Fuadi Fancy

Printed Name

Graduate Research Assistant

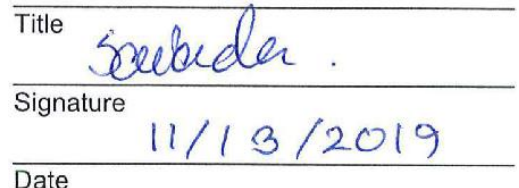

Date

Request agreed to by:

Saiada Fuadi Fancy

Lead Author Printed Name

Graduate Research Assistant

Lead Author Title
$\frac{\text { Lead Author Signature }}{11 / 13 / 2019}$

Date
Request approved by NACE:

\begin{tabular}{l}
\hline Printed Name \\
Lauren Reyes \\
Title \\
Publications Copyright Specialist \\
Signature \\
Lauren Reyes \\
Date \\
$11 / 14 / 2019$
\end{tabular}


VITA

\section{SAIADA FUADI FANCY}

\section{EDUCATION}

$2014-2019$

Ph. D., Civil Engineering

Department of Civil and Environmental Engineering

Florida International University,

Miami, Florida

$2011-2014$

M.Sc., Structural Engineering

Bangladesh University of Engineering and Technology,

Dhaka, Bangladesh

$2005-2009$

B.Sc., Civil and Environmental Engineering

Shah Jalal University of Science and Technology,

Sylhet, Bangladesh

\section{PUBLICATIONS}

S.F. Fancy, M. A. Sabbir, K. Lau, and D. DeFord. "Three-Coat and Epoxy Mastic Bridge Coating Systems in Adverse Environments." Journal of Protective Coatings and Linings. Vol.35, No.5, May 2018, Pp.36-45.

M. Farzad, S. F. Fancy, K. Lau, A. Azizinamini. "Chloride Penetration at Cold Joints of Structural Members with Dissimilar Concrete Incorporating UHPC." Infrastructures.

Vol. 5, Issue. 2, June 2019, Pp18.

S.F. Fancy, M. A. Sabbir, K. Lau, and D. DeFord. "Nano-Particle Enriched Epoxy Primer to Enhance the Durability of Highway Steel Bridges.” Structures Congress/2019.

S.F. Fancy, M. A. Sabbir, K. Lau, and D. DeFord. "Extended Connectivity of Zinc Pigments to Provide Enhanced Galvanic Coupling by Partial Replacement with Nanoparticles." NACE Corrosion/2019.

M. Farzad, S.F. Fancy, K.Lau, and A. Azizinamini. "Effect of Concrete Moisture on Macrocell Development in Repair of Reinforced Concrete Substructure with UHPC." NACE Corrosion/2019.

S.F. Fancy, K. Lau, and D. DeFord. "Mechanical Performance of Nano-Particle Enriched Zinc Rich Coatings." SSPC2019.

M. A. Sabbir, S. F. Fancy, K. Lau, and D. DeFord. "Update of Long-Term Durability of TDG Coating in Atmospheric Environment." NACE Corrosion Risk Management Conference/ June 11-13, 2018. 12pp. 
S. F. Fancy, M. A. Sabbir, K. Lau, and D. DeFord. "Corrosion Susceptibility of Nanoparticle Enriched Zinc Coating after Adverse Exposure during Repair." NACE Corrosion Risk Management Conference/ June 11-13, 2018. 16pp.

M. A. Sabbir, S. F. Fancy, K. Lau, and D. DeFord. "Assessment of Long-Term Durability of TDG Coated Steel.” NACE Corrosion/2018. Paper No. 11573.

S. F. Fancy, M. A. Sabbir, K. Lau, and D. DeFord. "Corrosion Durability of Three-Coat and Mastic Epoxy Coating Systems after Adverse Environmental Exposures during Coating Repair." SSPC2018. 8pp.

M. A. Sabbir, S. F. Fancy, K. Lau, and D. DeFord. "Assessment of Novel Coatings in Comparison to Conventional Zinc-Rich Coating for Bridge Application.” SSPC2018. 8pp.

S. F. Fancy, Md. A. Sabbir, K. Lau, and D. DeFord. "Corrosion Performance of NanoParticle Enriched Epoxy Primer for Marine Highway Bridge Application.” NACE Corrosion/2017. Paper No. 9539. 12pp.

M. A. Sabbir, S. F. Fancy, K. Lau, and D. DeFord. "Update on Corrosion Performance of CBPC Coatings in Aggressive Bridge Environments." NACE Corrosion/2017. Paper No. 9593. 12pp.

M. A. Sabbir, S. F. Fancy, K. Lau, and D. DeFord. "Characterizing Bulk Porosity of CBPC Coatings on Aggressive Bridge Exposure.” 3.1M.2.4. SSPC 2017.

S. F. Fancy, K. Lau, and D. DeFord. "Coating Response to Cathodic Polarization of Nanoparticle Enriched Epoxy Primer.” 1.1M.1.3. SSPC 2017. 